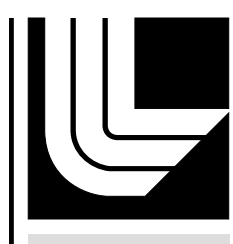

LAWRENCE LIVERM ORE N A TIONAL LABORATORY

TOWARD UNDERSTANDING DYNAMIC ANNEALING PROCESSES IN IRRADIATED CERAMICS

M. T. Myers

January 18, 2013 
This document was prepared as an account of work sponsored by an agency of the United States government. Neither the United States government nor Lawrence Livermore National Security, LLC, nor any of their employees makes any warranty, expressed or implied, or assumes any legal liability or responsibility for the accuracy, completeness, or usefulness of any information, apparatus, product, or process disclosed, or represents that its use would not infringe privately owned rights. Reference herein to any specific commercial product, process, or service by trade name, trademark, manufacturer, or otherwise does not necessarily constitute or imply its endorsement, recommendation, or favoring by the United States government or Lawrence Livermore National Security, LLC. The views and opinions of authors expressed herein do not necessarily state or reflect those of the United States government or Lawrence Livermore National Security, LLC, and shall not be used for advertising or product endorsement purposes.

This work performed under the auspices of the U.S. Department of Energy by Lawrence Livermore National Laboratory under Contract DE-AC52-07NA27344. 
Rev. 1.2

\title{
TOWARD UNDERSTANDING DYNAMIC ANNEALING PROCESSES IN IRRADIATED CERAMICS
}

\author{
A Dissertation \\ by \\ MICHAEL THOMAS MYERS
}

\author{
Submitted to the Office of Graduate Studies of \\ Texas A\&M University \\ in partial fulfillment of the requirements for the degree of \\ DOCTOR OF PHILOSOPHY
}

Approved by:

Chair of Committee, Lin Shao

Committee Members, Sergei Kucheyev

Sean McDeavitt

Haiyan Wang

Xinghang Zhang

Department Head, Yassin Hassan

May 2013

Major Subject: Nuclear Engineering

Copyright 2013 Michael Thomas Myers 
Rev. 1.2

\begin{abstract}
High energy particle irradiation inevitably generates defects in solids. The ballistic formation and thermalization of the defect creation process occur rapidly, and are believed to be reasonably well understood. However, knowledge of the evolution of defects after damage cascade thermalization, referred to as dynamic annealing, is quite limited. Unraveling the mechanisms associated with dynamic annealing is crucial since such processes play an important role in the formation of stable postirradiation disorder in ion-beam-processing of semiconductors, and determines the "radiation tolerance" of many nuclear materials. The purpose of this dissertation is to further our understanding of the processes involved in dynamic annealing. In order to achieve this, two main tasks are undertaken.

First, the effects of dynamic annealing are investigated in $\mathrm{ZnO}$, a technologically relevant material that exhibits very high dynamic defect annealing at room temperature. Such high dynamic annealing lead to unusual defect accumulation in heavy ion bombarded $\mathrm{ZnO}$. Through our work, the puzzling features that were observed more than a decade ago in ion-channeling spectra have finally been explained. We show that the presence of a polar surface substantially alters damage accumulation. Non-polar surface terminations of $\mathrm{ZnO}$ are shown to exhibit enhanced dynamic annealing compared to polar surface terminated $\mathrm{ZnO}$. Additionally, we demonstrate one method to reduce radiation damage in polar surface terminated $\mathrm{ZnO}$ by means of a surface modification. These results should further our efforts in the long-soughtafter goal of understanding complex radiation damage processes in non-amorphizable oxides.
\end{abstract}

Second, a pulsed ion beam method is developed and implement using Si as a 
prototypical ceramic target. Such a method is shown to provide a novel experimental method for direct extraction of dynamic annealing parameters. The relaxation times and effective diffusion lengths of mobile defects during the dynamic annealing process play a vital role in damage accumulation. We demonstrate that these parameters dominate the formation of stable post-irradiation disorder. In $\mathrm{Si}$, a defect lifetime of $\sim 6 \mathrm{~ms}$ and a characteristic defect diffusion length of $\sim 30 \mathrm{~nm}$ are measured. These results should nucleate a number of future pulsed-beam studies of dynamic defect interaction processes in technologically relevant materials. In particular, understanding the length and time scales of defect interactions are essential for extending laboratory findings to nuclear material lifetimes and to the time scales of geological storage of nuclear waste.

The results of both, the pulsed beam method for measuring dynamic annealing parameters, as well as damage accumulation in various planes of $\mathrm{ZnO}$ bombarded by heavy ions, are discussed. The work presented in this dissertation should substantially further our understanding of dynamic annealing processes in ceramics. 
Rev. 1.2

\section{DEDICATION}

To my wife and my parents, thank you for your endless encouragement. 
Rev. 1.2

\section{ACKNOWLEDGEMENTS}

I would like to acknowledge Dr. Shao for his guidance support during my tenure at Texas A\&M University. I would like to thank my committee members, Dr. McDeavitt, Dr. Wang, and Dr. Zhang for their support over the course of the present work. An additional debt of gratitude is owed to my Lawrence Livermore National Laboratory mentor, Dr. Kucheyev, who spent countless hours helping me grow into a mature scientist. A sincere "thank you" to Dr. Charnvanichborikarn who spent many hours assisting with experimental design as well as performing the actual experiments at Lawrence Livermore National Laboratory. I would also like to thank my group members, colleagues, and collaborators, Dr. Aitkaliveya, Dr. Luo, Dr. Lee, M. S. Martin, D. Chen, M. Hollander, C. C. Wei, M. A. Myers, J. Wallace, and M. J. General for their help throughout the dissertation.

Finally, I would like to acknowledge the Lawrence Scholar Program at the Lawrence Livermore National Laboratory for funding the work performed in this dissertation. 


\section{NOMENCLATURE}

\begin{tabular}{|c|c|}
\hline $\mathrm{A}$ & Ampere \\
\hline $\mathrm{Al}_{2} \mathrm{O}_{3}$ & Aluminum Oxide \\
\hline $\mathrm{AlO}(\mathrm{OH})$ & Aluminum Oxide Hydroxide \\
\hline $\mathrm{BF}$ & Bright Field \\
\hline BP & Bulk Defect Peak \\
\hline $\mathrm{C}$ & Coulomb \\
\hline CdTe & Cadmium Telluride \\
\hline $\mathrm{CM}$ & Center of Mass \\
\hline $\mathrm{DA}$ & Dynamic Annealing \\
\hline DF & Dark Field \\
\hline DLTS & Deep Level Transient Spectroscopy \\
\hline DPA & Displacement Per Atom \\
\hline EDS & Energy Dispersive X-ray Spectroscopy \\
\hline $\mathrm{eV}$ & Electron Volt \\
\hline FIB & Focused Ion Beam \\
\hline FZ & Float Zone \\
\hline HAADF & High Angle Annular Dark Field \\
\hline HEIS & High Energy Ion Scattering \\
\hline IBA & Ion Beam Analysis \\
\hline IBM & Ion Beam Modification \\
\hline IED & Ionization Enhanced Diffusion \\
\hline IP & Intermediate Defect Peak \\
\hline
\end{tabular}




\begin{tabular}{|c|c|}
\hline $\mathrm{keV}$ & Kilo Electron Volt \\
\hline $\mathrm{KP}$ & Kinchin-Pease \\
\hline$L_{\mathrm{d}}$ & Diffusion Length \\
\hline LED & Light Emitting Diode \\
\hline LLNL & Lawrence Livermore National Laboratory \\
\hline $\mathrm{MeV}$ & Mega Electron Volt \\
\hline ME & Molecular Effect \\
\hline MD & Molecular Dynamics \\
\hline $\mathrm{MgO}$ & Magnesium Oxide \\
\hline $\mathrm{MgAl}_{2} \mathrm{O}_{4}$ & Magnesium Aluminate Spinel \\
\hline NEC & National Electrostatics Corporation \\
\hline $\mathrm{nm}$ & Nanometer \\
\hline NRT & Norgett, Robinson and Torrens \\
\hline PKA & Primary Knock-on Atom \\
\hline $\mathrm{PL}$ & Photoluminescence \\
\hline $\mathrm{RBS} / \mathrm{C}$ & Rutherford Backscattering and Channeling Spectrometry \\
\hline $\mathrm{RT}$ & Room Temperature \\
\hline $\mathrm{Si}$ & Silicon \\
\hline $\mathrm{SiC}$ & Silicon Carbide \\
\hline $\mathrm{Si}_{3} \mathrm{~N}_{4}$ & Silicon Nitride \\
\hline SP & Surface Defect Peak \\
\hline SPER & Solid Phase Epitaxial Recrystallization \\
\hline SRIM & Stopping and Range of Ions in Matter \\
\hline SRP & Spreading Resistance Profiling \\
\hline
\end{tabular}


Rev. 1.2

STEM Scanning Transmission Electron Microscopy

Tau $(\tau)$ Defect Lifetime

TEM Transmission Electron Microscopy

TFD Thomas-Fermi-Dirac

TRIM Transport and Range of Ions in Matter

UN Uranium Nitride

$\mathrm{UO}_{2} \quad$ Uranium Oxide

XTEM Cross Sectional Transmission Electron Microscopy

$\mathrm{ZnO} \quad$ Zinc Oxide 


\section{TABLE OF CONTENTS}

Page

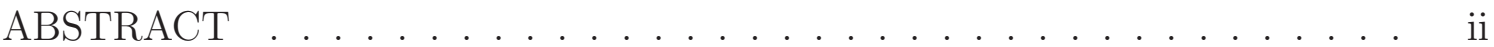

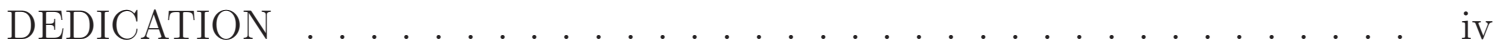

ACKNOWLEDGEMENTS . . . . . . . . . . . . . . . v v

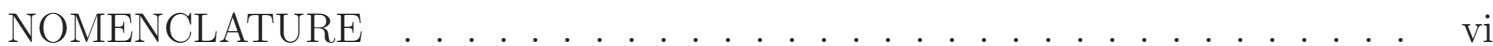

TABLE OF CONTENTS .................... . . ix

LIST OF FIGURES . . . . . . . . . . . . . . . . . . xi

LIST OF TABLES . . . . . . . . . . . . . . . . . . xviii

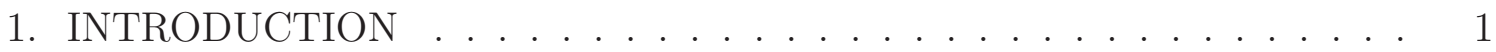

1.1 Dissertation scope . . . . . . . . . . . . . . . . . . . 1

1.1.1 Dynamic annealing in $\mathrm{ZnO}$. . . . . . . . . . . . . . . 2

1.1.2 Pulsed ion beams to probe dynamic annealing . . . . . . . . . 3

2. BACKGROUND ON ION-SOLID INTERACTIONS .......... 5

2.1 Ion acceleration . . . . . . . . . . . . . . 5

2.2 Interactions between ions and atoms . . . . . . . . . . . . . . . 8

2.2.1 Interatomic potentials . . . . . . . . . . . . 8

2.2.2 Scattering . . . . . . . . . . . . . . 10

2.2.3 Ion stopping and range . . . . . . . . . . . . . . . . . . . 11

2.2.4 Electronic stopping . . . . . . . . . . . . . . . . . 15

2.2.5 Nuclear stopping . . . . . . . . . . . . . . . . . . . 17

2.2.6 Primary knock-on atom displacements . . . . . . . . . . . . . 18

2.2.7 Displacement mean free path . . . . . . . . . . . . . . . 21

2.2.8 Thermal spike . . . . . . . . . . . . . . . . . . 22

2.2.9 Dynamic annealing . . . . . . . . . . . . . . . . . 24

2.3 Fluence effect . . . . . . . . . . . . . . . . . . . . . 26 


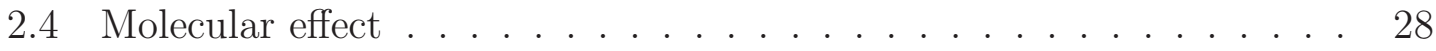

2.5 Flux effect . . . . . . . . . . . . . . . . . . . 28

3. CHARACTERIZATION METHODS ................. . . 31

3.1 High energy ion scattering and channeling . . . . . . . . . 31

3.2 Transmission electron microscopy . . . . . . . . . . . . . . . 35

4. HEAVY-ION-BOMBARDED ZINC OXIDE . . . . . . . . . . . . . 39

4.1 Introduction to radiation damage in ceramics . . . . . . . . . . 39

4.2 The role of the surface in heavy-ion-bombarded $\mathrm{ZnO} \ldots \ldots . . .46$

4.3 Defect microstructure in heavy-ion-bombarded (0001) ZnO . . . . 57

4.4 Tailoring radiation damage in (0001) $\mathrm{ZnO}$ by surface modification . . 66

4.5 Dynamic annealing differences in polar and non-polar $\mathrm{ZnO} \ldots . . .74$

4.6 Comparing the effect of surface modification in polar and non-polar $\mathrm{ZnO}$ bombarded with heavy ions . . . . . . . . . . 88

5. PULSED-ION-BEAM IRRADIATION . . . . . . . . . . . . . . . . . 99

5.1 Introduction to pulsed-ion-beam irradiation $\ldots \ldots \ldots \ldots$

5.2 Parameters and model . . . . . . . . . . . . . . . . . . . 104

5.3 Experimental setup . . . . . . . . . . . . . . . . . . . . . . . . . . 114

5.4 Method to measure the time constant of dynamic annealing in silicon at room temperature . . . . . . . . . . . . . . 120

5.5 Method to measure the diffusion length of dynamic annealing in silicon at room temperature . . . . . . . . . . . . . . 131

6. SUMMARY . . . . . . . . . . . . . . . . . . . . . . . . 141

6.1 Heavy-ion-bombarded $\mathrm{ZnO} \ldots \ldots \ldots \ldots$

6.2 Pulsed-ion-beam irradiation . . . . . . . . . . . . . . . . 144

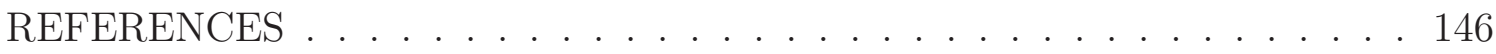

APPENDIX A. DECHANNELING CALCULATION CODE . . . . . . . . 160

APPENDIX B. AUSPICES STATEMENT . . . . . . . . . . . 167 
Rev. 1.2

\section{LIST OF FIGURES}

FIGURE

Page

2.1 A schematic of the 4MV ion accelerator at Lawrence Livermore National Laboratory. Shown (from upstream to downstream) are tank and high voltage terminal in blue followed by a quadrupole lens and mass analyzer. There are two beam lines: IBM beam line shown at the bottom with faraday cups and raster scanners and an IBA beam line shown at the top. . . . . . . . . . . . . . .

2.2 An illustration of a scattering event as experienced by the projectile and target in the center of mass coordinates. . . . . . . . . . . . 12

2.3 A diagrammatic representation of electronic and nuclear collisions between an incident ion and target atoms. . . . . . . . . . . . . . . 13

2.4 Illustration of the regimes of dominance for nuclear and electronic stopping as a function of energy. At sufficiently high energies the projectile is considered to be stripped of electrons and fully ionized, whereas in the low energy regime the projectile is considered neutral.

2.5 The Firsov quasi molecule, depicting the P-region, T-region and Firsov plane formed during electron stopping in the limit of $v<v_{0} Z^{2 / 3} \ldots$

2.6 Graphical depiction of the Kinchin-Pease model which depicts the number of displacements based on the energy distribution function $\left(F_{d}\right)$, the displacement energy $\left(E_{d}\right)$, and the fluence $(\Phi) \ldots \ldots \ldots$

2.7 Illustration of the displacement spike as a result of collisions occurring with a displacement mean free path on the order of the average atomic spacing. . . . . . . . . . . . . . . .

2.8 A schematic showing the parameters associated with DA for an individual ion impinging on a sample surface. . . . . . . . . . . . 25

2.9 A schematic of the fluence effect showing increased defect accumulation in overlapping damaged regions. . . . . . . . . . . . 27

2.10 A schematic of the flux effect showing increased defect accumulation due to similar vacancy concentrations in damaged regions within the diffusional zone. . . . . . . . . . . . . . . . . . . . . 
3.1 Typical arrangement for Rutherford backscattering spectrometry performed in the channeling orientation. (a) Illustrates the use of both a glancing detector for enhanced near surface resolution (typically $\lesssim$ $15^{\circ}$ ), and a backscatter detector which provides enhanced depth resolution (typically $\sim 164^{\circ}$ ). (b) Depicts an $\alpha$ particle (i.e. a He atom) scattering off of an atom that lies in the channel (interstitial). . . .

3.2 Illustration of the working principles and components of a transmission electron microscope . . . . . . . . . . . . . . .

4.1 Wurtzite crystal structure for $\mathrm{ZnO}$. Dark grey spheres represent oxygen atoms and light grey spheres represent zinc atoms. The polar zinc terminated face (0001) is at the top of the figure. . . . . . . . . .

4.2 Unreconstructed surfaces of (a) polar (0001), and non-polar (b) (112̄0) and (c) (1010) ZnO. Oxygen atoms indicated by red spheres and zinc atoms by blue spheres. The color of surface atoms are indicated in the legend of each panel for both zinc and oxygen atoms. . . . . . . .

4.3 (a) Normalized TRIM-code predicted profiles of lattice vacancies (closed symbols) and implanted Xe atoms (open symbols) for $500 \mathrm{keV}$ Xe ion irradiation of $\mathrm{ZnO}$ at $7^{\circ}$ and $60^{\circ}$ off the surface normal. The vertical scale is linear and is in arbitrary units. Concentrations of excess vacancies are also shown by lines without symbols. Vertical dash lines mark the maxima of vacancy profiles, $R_{p d}$ 's, for both implantation angles. Circle and square symbols represent results for $7^{\circ}$ and $60^{\circ}$ implants, respectively. . . . . . . . . . . . . .

4.4 (a)-(d) Depth profiles of relative disorder in (0001) $\mathrm{ZnO}$ bombarded at RT by $500 \mathrm{keV} \mathrm{Xe}$ ions at $7^{\circ}$ and $60^{\circ}$ off the [0001] direction to different fluences, indicated in the legends in units of DPA. For clarity, only every 20th experimental point has a symbol. In all five panels, circle and square symbols represent results for $7^{\circ}$ and $60^{\circ}$ implants, respectively. Vertical dash lines mark the maxima of vacancy profiles, $R_{p d}$ 's, for both implantation angles. . . . . . . . . . . .

4.5 Fluence dependencies of (a) (left scale) relative disorder at the maximum of the bulk defect peak (BP), (a) (right scale) areal defect concentrations in the surface peak (SP) and the intermediate peak (IP) of disorder, and the position of (b) (left scale) the BP maximum and (b) (right scale) the IP from the sample surface. The legend in (b) applies to both panels. . . . . . . . . . . . . . . . 
4.6 (a) XTEM image (slightly under-focused, around $11 \overline{2} 0$ zone axis) of $\mathrm{ZnO}$ bombarded to 5 DPA. Overlay in (a) is the depth profile of relative disorder measured by RBS/C, where only every 5th experimental point is depicted for clarity. The depth scale of the RBS/C spectrum also applies to the image in (a). (b)-(c) Higher resolution XTEM images of the same sample. Image (b) is in focus, around the $11 \overline{2} 0$ zone axis. Image (c) is under-focused, slightly off the $11 \overline{2} 0$ zone axis. The sample surface is shown by black triangles. The $c$-axis shown by a horizontal white arrow in (a) applies to all three images. Bombardment was done at RT with $500 \mathrm{keV}$ Xe ions at $60^{\circ}$ off the [0001] direction.

4.7 (a) XTEM image (in-focus, around $11 \overline{2} 0$ zone axis) of $\mathrm{ZnO}$ bombarded to 15 DPA. Overlay in (a) is the depth profile of relative disorder measured by RBS/C, where only every 5th experimental point is depicted for clarity. (b) HAADF STEM image of the 15 DPA sample. (c) Concentration of $\mathrm{Zn}$ as a function of depth obtained from a STEM/EDS line scan of (b). Sample surface is shown by triangles in (a) and (b). The $c$-axis is marked with a horizontal white arrow, and the depth scale of the RBS/C spectrum applies to both images in (a) and (b). Bombardment was done at RT with $500 \mathrm{keV}$ Xe ions at $60^{\circ}$ off the

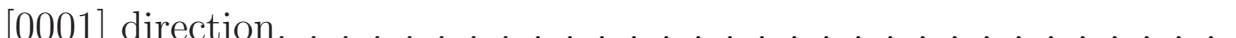

4.8 (a) XTEM image (slightly off the $11 \overline{2} 0$ zone axis) of ZnO bombarded to 15 DPA. (b) Higher magnification Fourier filtered XTEM image of (a) with the left edge starting at a depth of $\sim 2 \mathrm{~nm}$ from the sample surface. (c) Fourier filtered XTEM image of the region defined by the white square in (a). (d) Inverse Fourier transform of (c) with only $\pm(0002)$ reflections selected. The $c$-axis shown by a horizontal white arrow in (a) applies to all images. The sample surface is shown by a black triangle in (a). Bombardment was done at RT with $500 \mathrm{keV}$ Xe ions at $60^{\circ}$ off the $[0001]$ direction. . . . . . . . . . .

4.9 Depth profiles of relative disorder in (0001) $\mathrm{ZnO}$ bombarded at RT by $500 \mathrm{keV}$ Xe ions at $60^{\circ}$ off the surface normal to (a) 5 and (b) 15 DPA. For clarity, only every 10th experimental point has a symbol. In both (a) and (b), circle and square symbols represent results for bare $\mathrm{ZnO}$ (denoted as "bare") and $\mathrm{ZnO}$ coated with an $\sim 7$-nm-thick $\mathrm{AlO}(\mathrm{OH})$ layer (denoted as "coated"), respectively. Centers of surface, intermediate, and bulk defect peaks are indicated by arrows and labeled SP, IP, and BP, respectively. Normalized vacancy generation profiles predicted by TRIM-code calculations are also shown in (a) by open symbols, with circles for bare $\mathrm{ZnO}$ and squares for coated $\mathrm{ZnO}$. 
4.10 XTEM images of (0001) ZnO with [(a)-(c)] and without [(d),(e)] an $\sim 7$-nm-thick $\mathrm{AlO}(\mathrm{OH})$ layer deposited on top of the $\mathrm{ZnO}$ surface before ion bombardment. The sample before irradiation is shown in (a). Samples irradiated to 5 DPA are shown in (b) and (d), while (c) and (e) are images of samples irradiated to 15 DPA. All images were taken around the $11 \overline{2} 0$ zone axis, and irradiation was performed at RT by $500 \mathrm{keV} \mathrm{Xe}$ ions at $60^{\circ}$ off the surface normal. The (0001) $\mathrm{ZnO}$ surface is marked by a horizontal white line with an arrow in $(\mathrm{a}-\mathrm{c})$

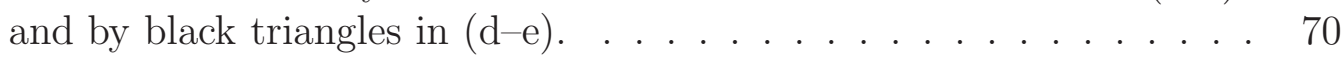

4.11 Atomic models of the crystallographic orientations and channels presented to the ion beam for the (a) [0001], (b) [112̄0], and (c) [1010] surface normal directions in $\mathrm{ZnO}$. White spheres represent $\mathrm{Zn}$ atoms while black spheres represent $\mathrm{O}$ atoms in (a-c). [114] (d) The (0001), $(1 \overline{1} 0)$, and $(10 \overline{1} 0)$ crystallographic planes in the wurtzite structure, shown as green, blue, and red, respectively. . . . . . . . . . . . . .

4.12 (a-d) Depth profiles of relative disorder measured by RBS/C in (0001), $(1 \overline{1} 0)$, and $(10 \overline{1} 0) \mathrm{ZnO}$ to increasing DPA. Bombardment was performed at RT with $500 \mathrm{keV}$ Xe ions at $7^{\circ}$ off the [0001], [112̄0], and [1010] directions, respectively. For clarity, every 15th experimental point is depicted. The legend in (a) applies to all four panels. . . . .

4.13 Fluence dependencies of the maximum relative disorder in (0001),

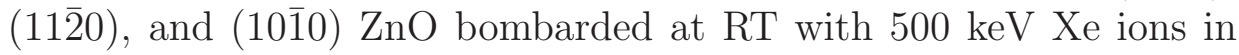
(a) the bulk defect peak, and (b) the surface peak. The legend in (a) applies to both panels. ................ 84

4.14 Depth profiles of relative disorder measured by RBS/C in (a) $C$-plane

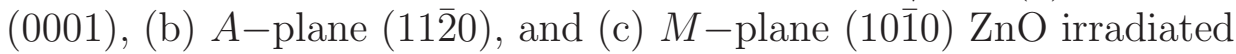
to $15 \mathrm{DPA}$ for both bare (i.e. uncoated) and $\mathrm{Al}_{2} \mathrm{O}_{3}$ surface modified (coated) samples. In $(\mathrm{a}-\mathrm{c})$ bombardment was performed at RT with

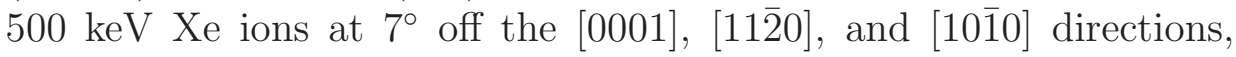
respectively. The zinc $(\mathrm{Zn})$, oxygen $(\mathrm{O})$, and aluminum $(\mathrm{Al})$ peaks, marked by vertical arrows in (c), and the the legend in (c) apply to all three panels. Note, an $\mathrm{Al}$ peak is only present in coated samples. For clarity, every 20th experimental point is depicted. . . . . . . . . . 
4.15 Maximum relative disorder in (a) the surface peak (SP) and (b) the bulk peak (BP) for $C$-plane (0001), $A$-plane (1120), and $M$-plane (1010) $\mathrm{ZnO}$ irradiated to $15 \mathrm{DPA}$ for both bare (i.e. uncoated) and $\mathrm{Al}_{2} \mathrm{O}_{3}$ surface modified (coated) samples. The values in (a) and (b) correspond to the maximum values in each of the surface and bulk peaks, respectively. Bombardment was performed at RT with $500 \mathrm{keV}$

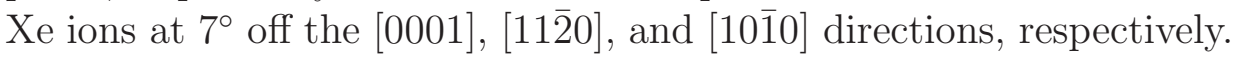
The lines connecting bare and coated data points (for each surface orientation) are depicted to clarify the trend. . . . . . . . . . . . .

5.1 Schematic representation of the pulsed ion beam experiment. (a) The fluence per pulse, $\Phi_{\text {pulse }}$, is $t_{\text {on }} \cdot \phi_{\max }$. (b) Linear defect production during $t_{o n}$ and dynamic defect annealing during $t_{\text {off }}$ are depicted. . . 105

5.2 Illustration of the number of stable lattice defects, $N_{d}$, after irradiation as a function of increasing $t_{o n}$. Applicable for the case of $\phi_{\max }, t_{\text {off }}$ fixed and $t_{\text {off }} \gg \tau$. The three regimes are denoted as $I, I I$, and $I I I$, illustrating the cases of $t_{\text {on }}<\tau, t_{\text {on }} \sim \tau$ and $t_{\text {on }}>\tau$, respectively. Three different damage annealing efficiencies, $\gamma$, are shown to illustrate differences between various ion/energy combinations. . . . . . . . . . 109

5.3 (a) Schematic of the number of stable lattice defects, $N_{d}$, after irradiation as a function of increasing $\Phi_{\text {pulse }}$ in the limit of $t_{\text {off }}>\tau$. Two curves $t_{o f f_{2}}$ and $t_{o f f_{1}}$ show differences in $t_{o f f}$. The distance over which defects leave the initial cascade is denoted as $L$. (b) Pictorially shows cascade overlap. Black regions represent the small amorphous zones, and gray regions represent areas that are partially damaged. . . . . . 112

5.4 A diagram of the major components in the pulsed ion irradiation facility which was constructed and operated at Lawrence Livermore National Laboratory for the experiments performed in this section. For clarity, only one set of electrostatic deflection plates is shown. In the actual setup, there were four plates as shown in Fig 5.5. Charge collection was performed using the actual sample surface which formed a portion of the Faraday cup arrangement. The suppression ring was biased to 180 volts to eliminate the role of secondary electrons in the charge integration. Actual sample holder setup is shown in Fig 5.6. 
5.5 A diagram of the connections to the parallel plates (shown in orange) which performed beam pulsing and wiggling. The aperture across which the ion beam was pulsed is shown in red with the ion beam shown as blue. In addition to the first aperture, there is a second exit aperture, shown in Fig 5.4 which would be directly downstream and therefore not visible in this schematic. . . . . . . . . . . . . . 117

5.6 A schematic drawing (both top and side view) of the sample holder and Faraday cage used in the pulsed beam setup. . . . . . . . . 118

5.7 Selected depth profiles of relative disorder in Si bombarded at RT by $500 \mathrm{keV}$ Ar ions (a) with a continuous beam with a constant flux of $1.2 \times 10^{13} \mathrm{~cm}^{-2} \mathrm{~s}^{-1}$ to various fluence (given in the legend in units of $10^{14} \mathrm{~cm}^{-2}$ ), (b) with a continuous beam to the same fluence of $2.4 \times 10^{14} \mathrm{~cm}^{-2}$ with different fluxs (given in the legend in units of $10^{12} \mathrm{~cm}^{-2} \mathrm{~s}^{-1}$ ), and (c) with a pulsed beam with different values of $t_{o f f}$ (given in the legend in units of $10^{-3} \mathrm{~s}$ ) and all the other parameters fixed (fluence $=2.4 \times 10^{14} \mathrm{~cm}^{-2}, t_{o n}=1 \mathrm{~ms}$, and $F_{\text {on }}=1.2 \times 10^{13}$ $\mathrm{cm}^{-2} \mathrm{~s}^{-1}$ ). The inset in (c) shows a schematic of the time dependence of the flux for pulsed beam irradiation, defining $t_{o n}, t_{o f f}$, and $F_{o n}$. . .

5.8 Fluence dependence of relative disorder at the maximum of the bulk defect peak for Si bombarded at room temperature by $500 \mathrm{keV}$ Ar ions with a flux of $1.2 \times 10^{13} \mathrm{~cm}^{-2} \mathrm{~s}^{-1}$ [based on ion channeling data such as shown in Fig. 5.7(a)]. The dash line shows a SRIM-code-predicted [20] dependence, taking into account damage saturation upon amorphization. The star denotes the fluence used in studies of defect dynamics.

5.9 Dependence of relative disorder at the maximum of the bulk defect peak on the average flux, $F_{\text {avg }}$ (for both continuous and pulsed beam irradiation) [bottom axis] and on $t_{\text {off }}$ (for pulsed beam irradiation) [top axis] for Si bombarded at room temperature by $500 \mathrm{keV}$ Ar ions to a fluence of $2.4 \times 10^{14} \mathrm{~cm}^{-2}$. For pulsed experiments, the maximum flux was $1.2 \times 10^{13} \mathrm{~cm}^{-2} \mathrm{~s}^{-1}$ and $t_{\text {on }}$ [defined in the inset of Fig. 5.7(c)] was $1 \mathrm{~ms} . \ldots \ldots \ldots$. . . . . . . . . . . . . . . . 127

5.10 Dependence of relative disorder at the maximum of the bulk defect peak on the passive portion of the beam $t_{\text {off }}$, with a fitting curve of the second order rate equation, discussed in the text, shown by a dash line. Error bars correspond to peak-to-peak noise in RBS/C-derived disorder profiles such as shown in Fig. 5.7(c). . . . . . . . . . . . 130 
5.11 Selected depth profiles of relative disorder in Si bombarded at room temperature by $500 \mathrm{keV}$ Ar ions with a pulsed beam with different values of $t_{\text {on }}$ (indicated in the legend) and all the other parameters fixed (total fluence $=2 \times 10^{14} \mathrm{~cm}^{-2}, t_{\text {off }}=100 \mathrm{~ms}$, and $F_{\text {on }} \approx 1.6 \times 10^{13}$ $\mathrm{cm}^{-2} \mathrm{~s}^{-1}$ ). The inset shows a schematic of the time dependence of the flux, defining $t_{\mathrm{on}}, t_{\mathrm{off}}, F_{\mathrm{on}}$ (the maximum instantaneous flux), and $\Phi_{\text {pulse }}$ (the fluence per pulse). . . . . . . . . . . . . 135

5.12 Dependence of relative disorder at the maximum of the bulk defect peak in Si bombarded at room temperature by a pulsed beam of 500 $\mathrm{keV}$ Ar ions on the duration of the active part of the cycle, $t_{\mathrm{on}}$, and all the other parameters fixed as in Fig. 5.11. The maximum damage level produced by a continuous beam $\left(t_{\text {off }}=0\right)$ is also shown. A critical value of $t_{\text {on }}$ above which the damage level is rapidly increases is labeled as $t_{\mathrm{on}}^{L_{\mathrm{d}}}$ and marked by an arrow. The inset shows a schematic of cascades in a slice made perpendicular to the beam direction, defining parameters $R_{\text {ballistic }}$ (the average radius of ballistic cascades), $L_{\mathrm{d}}$ (the characteristic defect diffusion length), and $L_{\text {overlap }}$ (the average lateral distance between the centers of collision cascades in one pulse). . . . 138 
Rev. 1.2

\section{LIST OF TABLES}

\section{TABLE}

4.1 A summary of the pertinent irradiation conditions used to perform irradiation of (0001) $\mathrm{ZnO}$ at RT with $500 \mathrm{keV} \mathrm{Xe}^{+}$ions to variable fluences at two incident ion angles. . . . . . . . . . . . . . . . 48

4.2 Irradiation conditions and relevant parameters used to perform irradiation of (0001) ZnO at RT with $500 \mathrm{keV} \mathrm{Xe}^{+}$ions to variable fluences.

* Denotes that this data has been reported previously. . . . . . . . . . 58

4.3 For both coated and uncoated (0001) ZnO, a summary of the irradiation conditions used to perform RT bombardment with $500 \mathrm{keV} \mathrm{Xe}$ ions to 5 and $15 \mathrm{DPA} .{ }^{\star}$ Denotes that this data has been reported previously. . . . . . . . . . . . . . . 71

5.1 A summary of the components, their bias voltage, and their purpose in the pulsed-ion-irradiation setup used to collect charge (i.e. calculate the fluence delivered) as shown in Figs. 5.4, 5.5 and 5.6. . . . . . . . . 120

5.2 A description of the plates (shown in Fig. 5.5), their bias voltage, voltage range, frequency, and the type of waveform used for the pulsedion-irradiation setup shown in Fig. 5.4. . . . . . . . . . . . 120 
Rev. 1.2

\section{INTRODUCTION}

The topic of dynamic defect annealing under irradiation arguably remains one of the least understood phenomena in all of radiation materials science. Despite many decades of research, the length and time scales of defect interaction processes have remained elusive. This is due, in part, to the fact that information on these quantities requires very precise experimental measurements and techniques.

Although radiation materials science encompasses radiation effects in various materials, the efforts contained in this dissertation focus solely on ceramics, and in particular, DA (DA) in selected ceramics. The basic layout of this dissertation consists of a brief background on ion-solid interactions (Section 2), a section on the characterization methods (Section 3) followed by two sections which comprise the bulk of the research which has been performed (Section 4 and 5). Section 4 and 5 each begin with an introduction and background into the topics presented. My contribution consists of those two sections followed by a synopsis. It should be noted that, for the most part, those two sections contain a collection of peer reviewed journal articles which have been previously published and adapted for this dissertation.

\subsection{Dissertation scope}

As previously stated, the overarching theme of this dissertation is related to the phenomenon of DA in ceramics. However, this is a broad topic and as such, two concise topics related to DA phenomena are investigated in detail. The work presented herein represents two parallel endeavors performed simultaneously. In fact, these two sections could be taken together as a broad study focused on uncovering the peculiarities of DA, or separately, with each focused on in-depth discussions pertinent to each material studied. 
The two main topics which are covered in this dissertation are:

1. Radiation damage buildup in a material with high technological importance, i.e. $\mathrm{ZnO}$, which has incredibly high $\mathrm{DA}$. As a result of the high $\mathrm{DA}$ at room temperature (RT), bombardment with heavy ions produces highly unusual damage buildup behavior. Section 4 contains the works performed in an attempt to understand the effect of the surface on DA in this class of materials, namely, those materials which have highly ionic bonding, are non-amorphizable, and exhibit high dynamic defect annealing at RT.

2. A series of novel pulsed beam experiments have been performed which enable measurement of the time constants and diffusion lengths associated with DA. Section 5 contains a series of experiments which are a first of their kind and have the potential to open up an entirely new field of radiation materials science.

In addition, the following two sub-sections provide a very brief introduction to the topics covered. For further reading on either of the two topics, one is referred to a longer discussion in the respective sections and references contained therein.

\subsubsection{Dynamic annealing in $\mathrm{ZnO}$}

It is well established that, at $\mathrm{RT}, \mathrm{ZnO}$ exhibits strong $\mathrm{DA}[1]$. As a result, radiation generated defects are mobile at RT and high-dose irradiation with heavy ions does not render $\mathrm{ZnO}$ amorphous at RT. Ion-beam-produced disorder has been intensively investigated in $\mathrm{ZnO}$ [2-12]. For a wide range of irradiation conditions, the level of stable post-implantation disorder in the $\mathrm{ZnO}$ crystal bulk depends linearly on the concentration of ballistically generated lattice displacements [4-6]. However, the buildup of damage in the near surface region is incredibly complex. In particular, an anomalous intermediate defect peak (IP) has been observed in depth profiles of 
lattice disorder in (0001) $\mathrm{ZnO}$ irradiated with heavy or cluster ions and measured by high-resolution Rutherford backscattering and channeling (RBS/C) [4, 6, 11-13]. The IP is located at a depth between the expected surface and bulk peaks of disorder.

Despite a number of systematic studies, it is still unclear the exact mechanism by which the IP forms and further studies aimed at understanding such intriguing and complex phenomena are currently needed. Section 4 is dedicated to numerous heavy-ion-irradiation experiments in $\mathrm{ZnO}$ which attempt to elucidate the role of $\mathrm{DA}$, the surface, and various other parameters on IP formation.

\subsubsection{Pulsed ion beams to probe dynamic annealing}

Understanding relaxation length- and time-scales of defects that dominate the stable post-implantation lattice disorder is of fundamental importance. Currently, there is a large variation in the estimates of the time scales over which defects persist and the lengths over which they diffuse after damage cascade thermalization. The reason is simple, accurate measurements of defect relaxation times are difficult to ascertain experimentally, and simulations often result in simplistic models which are unable to access time scales ( $>1 \mathrm{~ms}$ ) relevant to the problem.

We demonstrate a method utilizing pulsed-ion-irradiation to measure the relaxation times and diffusion lengths of the defects that dominate post implantation disorder. Pulsed-ion-irradiation is an ideal method to probe the flux effect and is reminiscent of pump-probe measurements in optical studies. Pulsed irradiation has the benefit of allowing control over the spatial separation between individual damage zones and the amount of annealing that occurs between pulses $[14,15]$.

In the present work, a pulsed beam irradiation setup is constructed and operated to measure fundamental constants associated with DA. A broad theory is presented in an attempt to understand the experimental data. Our results suggest that DA 
Rev. 1.2

can be described in terms of a dominant defect relaxation time and diffusion length. By precisely controlling the parameters associated with pulsed ion beam irradiation, these constants have been extracted for Si bombarded at RT. A description of the pulsed beam setup used in this work, our pulsed beam theory, and the subsequent measurement of parameters associated with DA are the topic of Section 5. 
Rev. 1.2

\section{BACKGROUND ON ION-SOLID INTERACTIONS*}

The present section explains the physical description of ion-solid interactions, including accelerator systems, scattering, energy loss, and flux and fluence effects. A fundamental understanding of ion-solid interactions is necessary to understand the data presented in this dissertation. Therefore, an entire section is dedicated to the topic. However, the area of ion-solid interactions is very broad and encompasses a wide range of theories and applications. The use of ion beams takes many forms, from ion implantation, ion mixing, doping, surface modification, material characterization and many others. This section provides a brief overview to the topic of ion-solid interactions. For a complete review, consult one of the many books on the topic $[16-18]$.

\subsection{Ion acceleration}

For most applications involving ions, incident ions are produced in an ion source (either solid or gas) and are subsequently accelerated through an electric potential, otherwise known as an acceleration column. Mass separation is achieved using a magnetic field to steer only the desirable ion species (charge to mass ratio) toward the target chamber. To focus the beam, electrostatic or magnetic lenses are employed. The beam is then raster-scanned across the desired area using a technique, known as beam sweeping, which uses magnetic fields to steer the beam. Although many other forms and variations of accelerators exist, the schematic shown in Fig. 2.1 is the 4MV NEC ion accelerator operated in the Ion Beam Laboratory at Lawrence Livermore National Laboratory.

* Reprinted, in part, from M.T. Myers, MS Thesis, Texas A\&M University. 


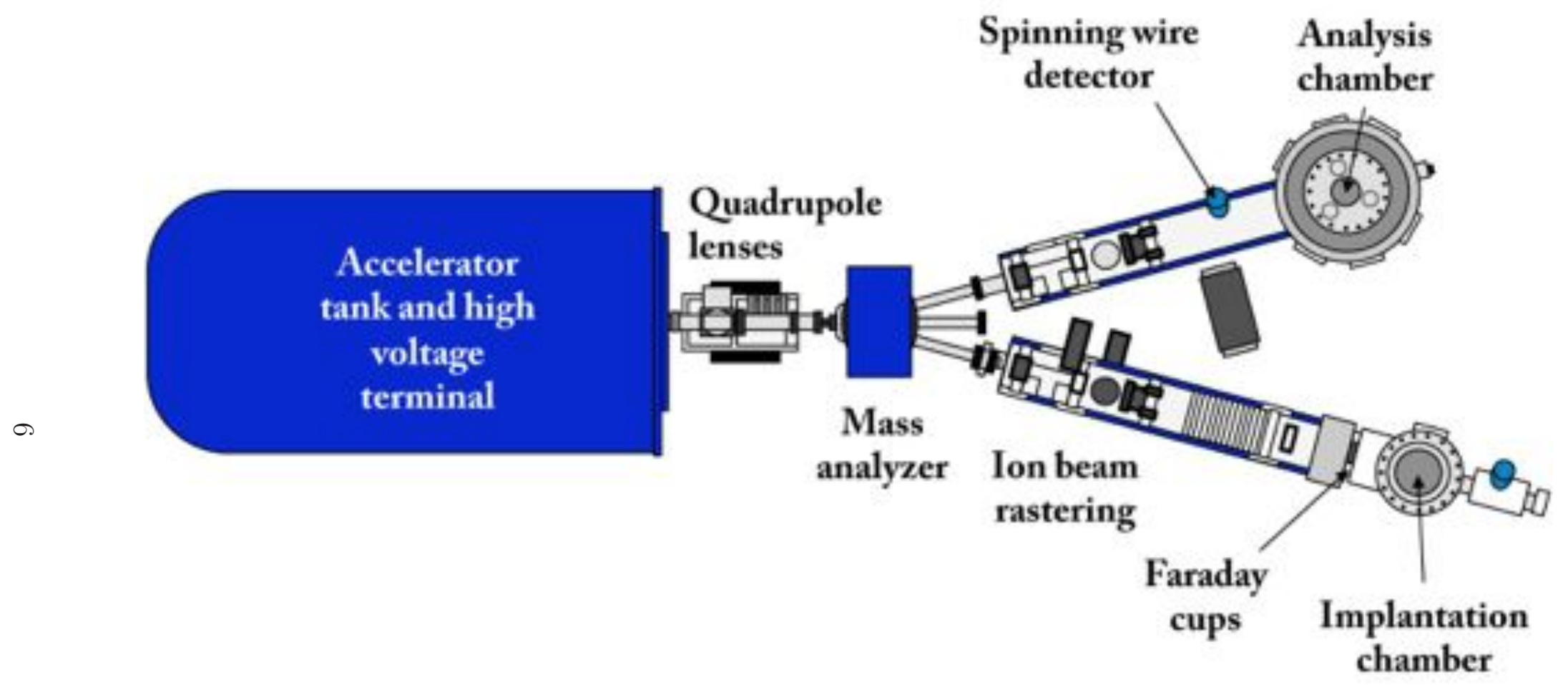

Figure 2.1: A schematic of the 4MV ion accelerator at Lawrence Livermore National Laboratory. Shown (from upstream to downstream) are tank and high voltage terminal in blue followed by a quadrupole lens and mass analyzer. There are two beam lines: IBM beam line shown at the bottom with faraday cups and raster scanners and an IBA beam line shown at the top. 
The entire system is held under near-vacuum conditions, typically less than $10^{-7}$ torr, to reduce the number of ion-gas collisions inside the system. These types of collisions result in ion energy loss, contaminating the energy spread of the incident ion beam. Assuming near-vacuum conditions, the beam will arrive at the target chamber as a current of ions of the same species and energy. The intensity of the beam is measured in SI units of amperes per square centimeter $\left(\mathrm{A} / \mathrm{cm}^{2}\right)$ and typical values, depending on the acceleration potential and the type of accelerator, can range from $10^{-3}$ to $10^{-6} \mathrm{~A} / \mathrm{cm}^{2}$.

The energy (more precisely, kinetic energy) of the bombarding ion is dictated by the charge state of the ion and the accelerating potential. To describe this energy, the unit of electron volt $(\mathrm{eV})$ is used, which is defined as the kinetic energy of a particle accelerated through a potential of one volt with the charge $e$, where $e$ is the charge on an electron. The SI unit of charge is the coulomb (C) and the charge on an electron is $1.602 \times 10^{-19} \mathrm{C}$. In the usual way, the known kinetic energy can be used to determine ion velocities such that, $E=\frac{1}{2} m v^{2}$, where $E$ is the kinetic energy, $m$ is the mass of the ion, and $v$ is the velocity. Typical values of ion energy are on the order of $\mathrm{keV}\left(10^{3} \mathrm{eV}\right)$ to $\mathrm{MeV}\left(10^{6} \mathrm{eV}\right)$.

The total fluence, $\Phi$, is then defined as the normalized number of ions implanted per area (typically per $\mathrm{cm}^{2}$ or per $\mathrm{m}^{2}$ ). This can be calculated from the beam intensity $\phi$, as

$$
\Phi=\int_{0}^{t_{f}} \phi(t) d t
$$

using the conversion from amperes to ions per second: $1 \mathrm{~A}=1 \mathrm{C} / \mathrm{s}=(1.602 \times$ $\left.10^{-19} \times n\right)^{-1}$ ion/s, where $n$ is the charge state of the ion. 
Rev. 1.2

\subsection{Interactions between ions and atoms}

As ions penetrate into the target, they make collisions with target atoms, creating primary knock-on atoms (PKA), which in turn have subsequent interactions. All collisions between ions and atoms, and atoms-atoms can be described through the use of interatomic potentials. Understanding the forces which dictate momentum and energy transfer are essential to determining ion range, damage creation and concentration of implanted species.

\subsubsection{Interatomic potentials}

The simplest potential model of the electrostatic potential between two point charges with charge $e$ separated by a distance $r$ is the Coulomb potential, given by

$$
V(r)=\frac{e^{2}}{r} .
$$

In the case of atoms, significantly more structure exists, such as a positively charged core surrounded by a negatively charged electron cloud. Equation 2.2 does not account for this level of detail and more complexity must be added to account for such features. In the hard sphere model, atoms are treated as billiard balls and are allowed to make strictly elastic collisions. This, also, is known not to be the case, as electron shells of two colliding atoms can, in fact, overlap and have significant contributions to the applied force. The Born-Mayer potential was introduced to account for the overlap of the valence shell electrons as the two atoms are brought nearer. The Born-Mayer potential is given by

$$
V(r)=A \exp \left(\frac{-r}{B}\right)
$$

where the constants $A$ and $B$ are determined experimentally. 
At distances much less than the Bohr radius, the Coulomb interaction will be dominant as the repulsive ion cores will be the primary forces between the two atoms. At distances on the order of the equilibrium separation distance between the two point charges, the Born-Mayer potential will most accurately resolve the forces between two atoms [19].

To account for the electronic screening that takes place in the region just outside of the Bohr radius, a screened Coulomb potential has been developed which takes the following form,

$$
V(r)=\left(\frac{Z_{1} Z_{2} e^{2}}{r}\right) \exp \left(\frac{-r}{a}\right)
$$

where $Z_{1}, Z_{2}$ are the atomic masses, and $a=0.8853 a_{0} /\left(Z_{1} Z_{2}\right)^{1 / 6}$ and $a_{0}$ is the Bohr radius.

A combination of the Born-Mayer potential and the screened Coulomb potential, each accounting for their respective regimes of dominance, is a good first approximation to the interatomic potential between two approaching atoms. Two other methods for calculating interatomic potentials are that of Frisov and also ThomasFermi-Dirac potentials (TFD). The approach used by Frisov accounts for the mutual approach of the nuclei and therefore takes into consideration changes in electronic energy levels, similar to that of screened Coulomb potential with a different functional form for the screening factor. The TFD potential takes into consideration the finite spatial distribution and density of the electron cloud.

Small changes to the screening function have been suggested by Bohr, Lindhard and Frisov, but all have been based on simple interatomic potentials. In an attempt to model all interatomic potentials using a single analytical form, Ziegler, Biersack and Littmark performed detailed calculations for 261 atom pairs to find a suitable 
potential [16]. They were able to derive what is known as the universal screening function together with their ZBL potential to fit the data. This model is widely used for calculations involving interatomic potentials.

\subsubsection{Scattering}

For the following discussion of elastic scattering, collisions are assumed to be violent collisions, that is, they are two-body collisions. The particles are treated classically, with electronic excitation only impacting the energy loss and not the collision dynamics. In this type of collision, one atom is considered to be initially at rest. The problem is, therefore, how to solve the equations which conserve energy and momentum for two-body collisions. Through these conservation equations, one can determine the energy transfer, momentum transfer and the scattering angle, typically working in the center of mass $(\mathrm{CM})$ frame. In the $\mathrm{CM}$ frame, the scattering angle will depend on the choice of interatomic potential, the ion energy and the impact parameter in the following way,

$$
\theta_{c}=\pi-2 \int_{r_{\min }}^{\infty} \frac{b \cdot d r}{r^{2}\left[1-\frac{V(r)}{E_{c}}-\frac{b^{2}}{r^{2}}\right]^{\frac{1}{2}}}
$$

where $\theta_{c}$ is the scattering angle, $v_{c}$ is the center of mass velocity, $v$ is the projectile velocity, $b$ is the impact parameter, $E_{c}$ is the cutoff energy, $V(r)$ is the potential [i.e. Equation 2.4] and the integration is performed from the line of closest approach, $r_{\min }$ to $\infty$. The impact parameter is the distance offset from the central force between the two bodies and a "magic" formula has been derived by Biersack (see [20] for more details) which enables quick calculation of the scattering angle and determination of the impact parameter.

By placing reasonable assumptions on the impact parameter value, a solution to 
the classical scattering integral can be obtained and, therefore, the energy transfer as a result of the scattering process can be obtained by solving,

$$
T=4 E \frac{m_{1} \cdot m_{2}}{\left(m_{1}+m_{2}\right)^{2}} \sin ^{2}\left(\frac{\theta_{c}}{2}\right)
$$

where $T$ is the energy transfer due to the collision, $m_{1}$ is the projectile mass, $m_{2}$ is the target mass, and $\theta_{c}$ is the center of mass scattering angle calculated by solving the scattering integral in Equation 2.5. Figure 2.2 depicts the parameters that are involved in CM scattering. For a more detailed description of classic two-body scattering, please refer to Ref. [16].

Bohr proposed the existence of two distinct regimes of energy loss due to elastic collisions. The first is the nuclear regime where the interaction is purely Coulombic with no screening, i.e. nucleus-nucleus collisions. The second is the electronic regime where one can use the harmonic oscillator model to represent the frequency of interactions between the electron cloud and the potential of the incoming ion. This allows for a natural cutoff value to the impact parameter and leads directly to the two main modes of energy transfer and stopping in solids.

\subsubsection{Ion stopping and range}

As an incident ion traverses through a material, the target atoms inside undergo many collisions with a given ion. The path that the ion takes depends on the crystal structure, energy, angle of incidence and is by nature, complicated. If the target is considered to be a random collection of atoms with no structure, then the ion track can be considered as a series of random scattering events that takes the ion in all directions. The total distance traversed by the particle is given by the range, $R$,

$$
R=\int_{E_{0}}^{0} \frac{1}{d E / d x} d E
$$




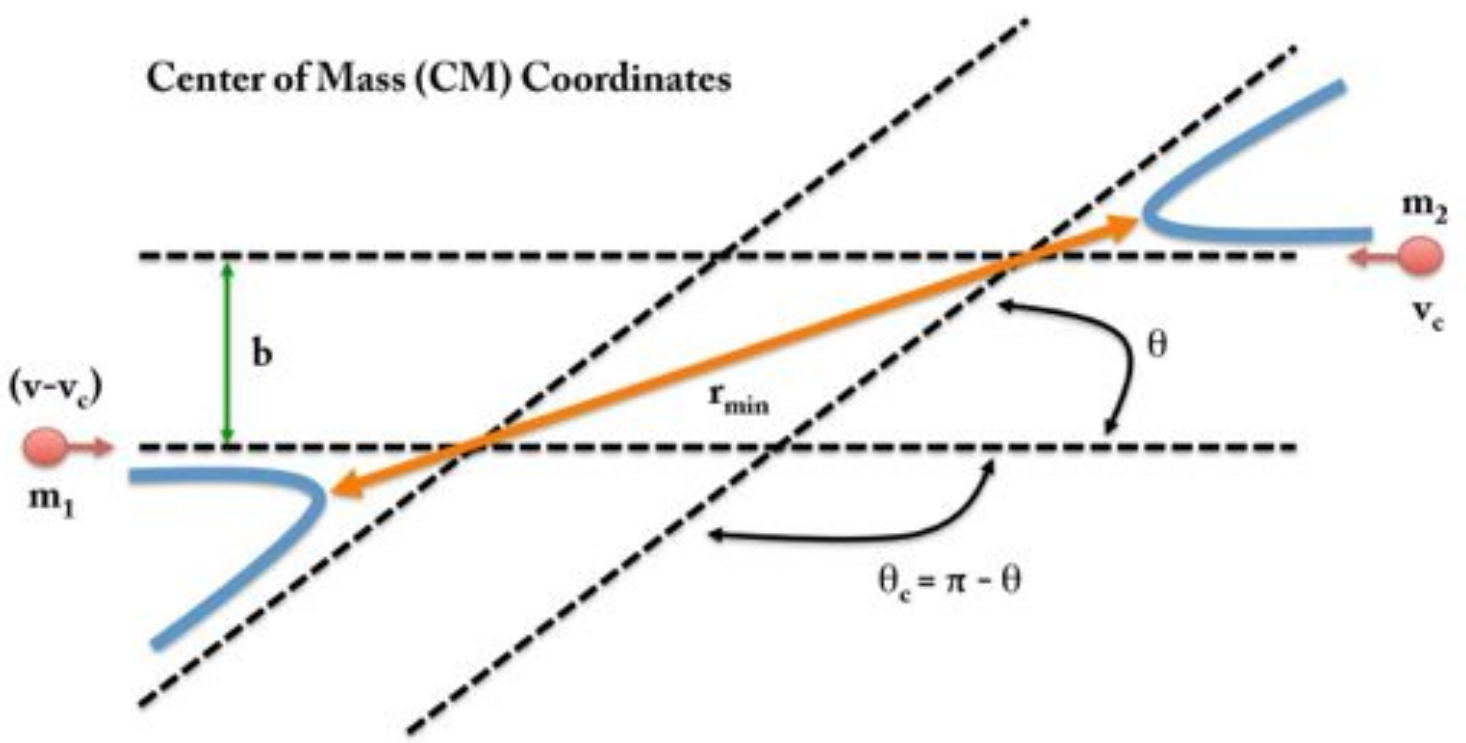

Figure 2.2: An illustration of a scattering event as experienced by the projectile and target in the center of mass coordinates.

where $E_{0}$ is the initial energy, and $d E / d x$ represents the energy loss per distance traveled. The more useful quantity for ion implantation is the projected range, $R_{p}$. Given a series of track histories, it is unlikely that any two will be identical, but the average perpendicular distance from the surface that an ion travels converges to a fixed value. This value is then given as the particles' projected range.

The projected range will follow a Gaussian distribution given the assumption of random collisions. Another term, called range straggling, $d R_{p}$, is often used to characterize the spread in the range distribution. The range and straggling depend on the details of the problem and the particular stopping mechanism. Projected range values are well tabulated and are the topic of many software simulations packages, such as the Transport and Range of Ions in Matter (TRIM), also commonly referred to as SRIM [20].

When an energetic ion is incident on a target of many atoms, it slows through collisions described above with target atoms. This process is known as stopping, and 


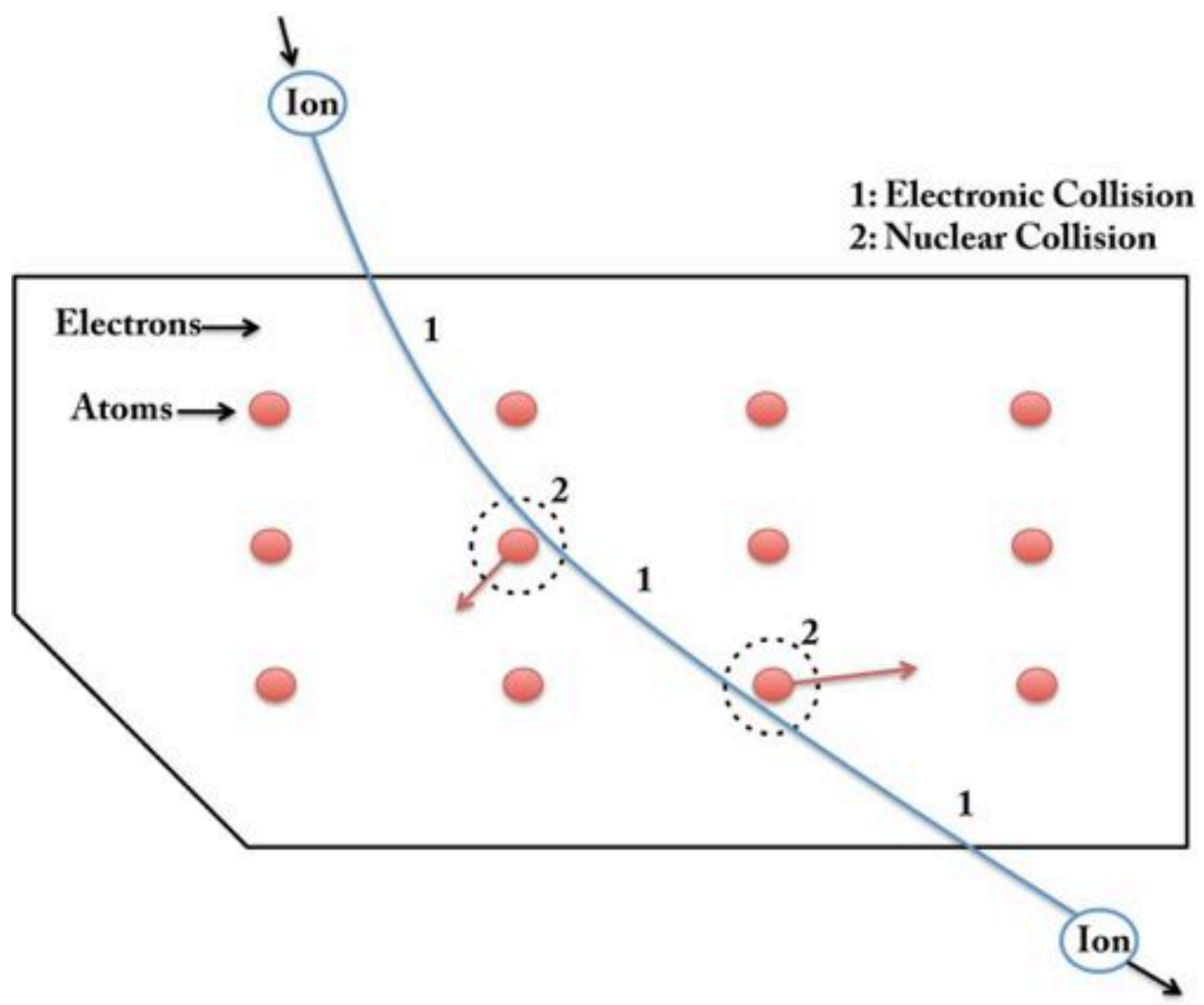

Figure 2.3: A diagrammatic representation of electronic and nuclear collisions between an incident ion and target atoms.

can be thought of as two interactions: interactions with target electrons and interactions with target nuclei, electronic and nuclear stopping, respectively. A depiction of the energy loss mechanisms is shown in Figure 2.3.

The nuclear stopping mechanism is dominant for low velocity projectiles, whereas electronic stopping is dominant for high velocity projectiles. Each mechanism is dependent on relevant parameters such as the ion mass and ion velocity. The relationship that exists for particle velocity and the dominant stopping mechanism is depicted in Figure 2.4. At very high ion velocities, the projectile is fully ionized as 


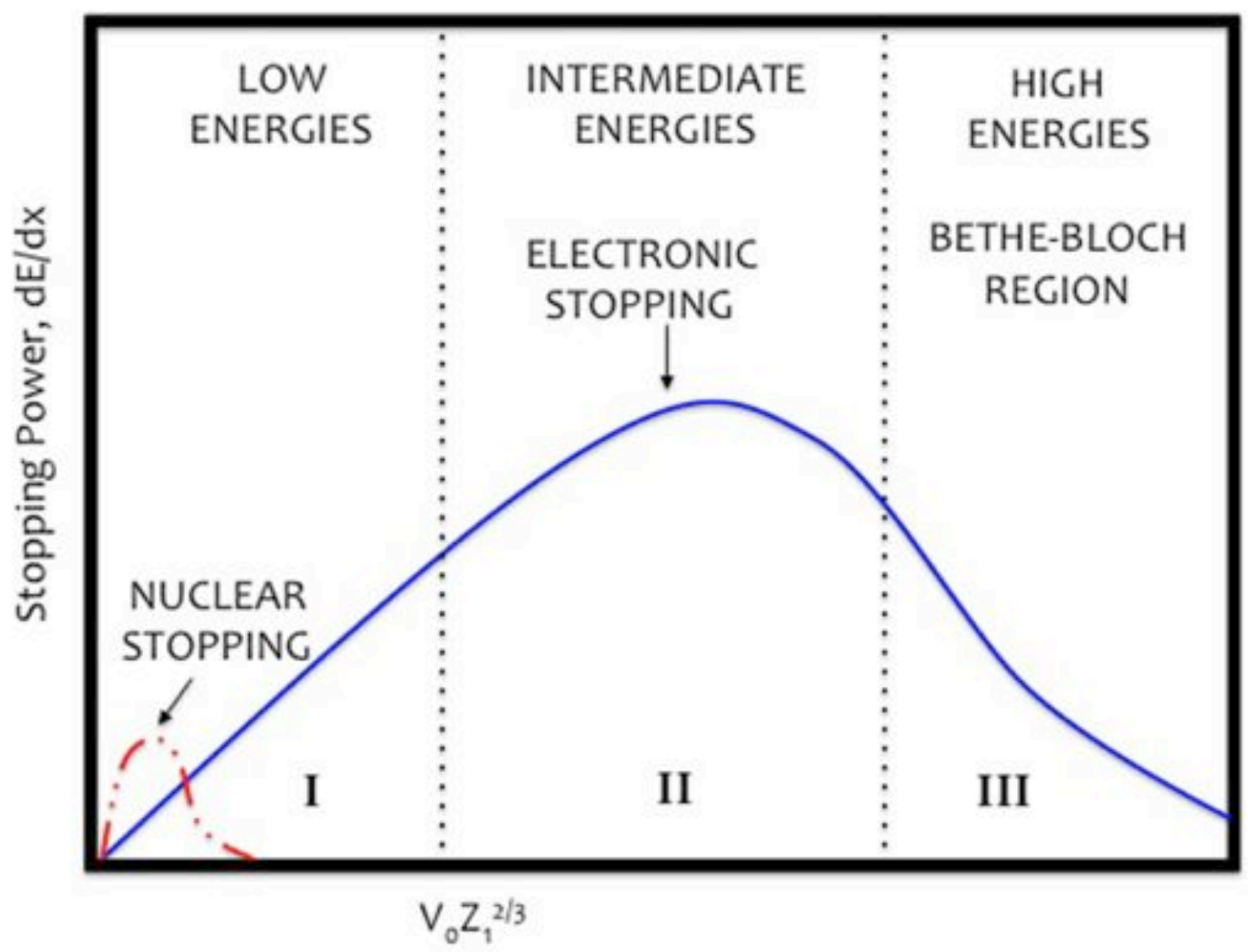

\section{Ion Velocity}

Figure 2.4: Illustration of the regimes of dominance for nuclear and electronic stopping as a function of energy. At sufficiently high energies the projectile is considered to be stripped of electrons and fully ionized, whereas in the low energy regime the projectile is considered neutral.

all of the electrons have been stripped (shown in Fig. 2.4 as Bethe-Bloch region) and few interactions take place until the ion energy is reduced. As the ion undergoes the process of slowing, typically through electronic interactions, it traverses the intermediate region where electronic stopping is dominant. As the ion continues to slow, the nuclear stopping component begins to become increasingly important.

Due to the nature of the two distinct regimes of ion stopping (i.e nuclear vs. electronic), it is therefore broken up into two components with $d E / d x$ being com- 
monly referred to as the stopping power, as seen in Equation 2.8. There exists a third component due to radiation, but for all intents and purposes, this term is often neglected. The stopping power is measured in terms of electron-volts per Angstrom $(\mathrm{eV} / \AA)$ and represents the energy loss per distance traversed in the material.

$$
\frac{d E}{d x}=\left.\frac{d E}{d x}\right|_{n}+\left.\frac{d E}{d x}\right|_{e}
$$

The process of electronic stopping consists of both elastic and inelastic processes, although the inelastic scattering dominates. This process involves excitation and ionization of electrons over the length of the ion path. This means that electronic energy losses can be viewed in terms of a continuous slowing down theory.

\subsubsection{Electronic stopping}

At high ion velocities, energy losses occur primarily through inelastic collisions with electrons in the form of electron excitation and ionization. In the limit of very high ion velocity, the ion can be viewed as a moving point charge traveling faster than the mean orbital velocities of electrons around the target atoms. This limit is known as the Bohr velocity, which is about $2.2 \times 10^{6} \mathrm{~m} / \mathrm{s}$, or about $1 \%$ the speed of light. Bohr himself suggested a correlation to determine the amount of ionization based on velocity and atomic number. The effective ion charge fraction is equal to $v / v_{0} Z^{2 / 3}$, where $v$ and $Z$ are the velocity and atomic number of the projectile. In the limit that the effective ion charge is equal to unity, then the ion is considered to be fully stripped of all electrons. In the other limiting case of effective charge equal to 0 , the ion is neutral and carries all of its electrons with it. This establishes a natural boundary between electronic stopping and nuclear stopping. The approximate velocity that

separates the two regimes is given by $v>v_{0} Z^{2 / 3}$ which is the velocity at which the ion becomes fully stripped of electrons. 
The nature of the purely Coulombic electronic stopping means that as the ion traverses the medium, energy is transferred to the target electrons with a maximum energy transfer for head on collisions with target electrons. The net energy transferred in such a collision will be highly dependent on the electron shell structure. At very high velocities, this method of slowing will be dominant, however collisions with target electrons does not induce target atom displacement.

As the effective velocity decreases, $v<v_{0} Z^{2 / 3}$, the ion starts to accumulate electrons as the ion is now traveling at velocities less than the mean orbital electron velocity. In this regime, the electronic stopping mechanism is still not completely understood and several theories prevail.

The model offered by Firsov [21] offers the following explanation. During the collision event, if the ion gains an electron, momentum is transferred to the electron. This additional momentum accelerates the electron to a given velocity, $v$, which reduces the energy of the incident projectile. This theory suggests that, for a short period of time, a quasi molecule exists in which electrons are temporarily "shared". An illustration of this process is seen in Figure 2.5, originally appearing described in Ref. [16].

Figure 2.5 shows a projectile traveling in the $x$ direction with velocity $v$ and separated in the $y$ direction by a distance $b$ from the central force. Firsov suggested that the quasi-molecule consisted of two zones, the P-zone and T-zone for the projectile and target, respectively. The plane that separates this two zones is termed the Firsov plane. By moving through the Firsov plane, the projectile gains electrons from the target. Determining the energy required for this situation to occur is equivalent to calculating the energy loss by the projectile. 


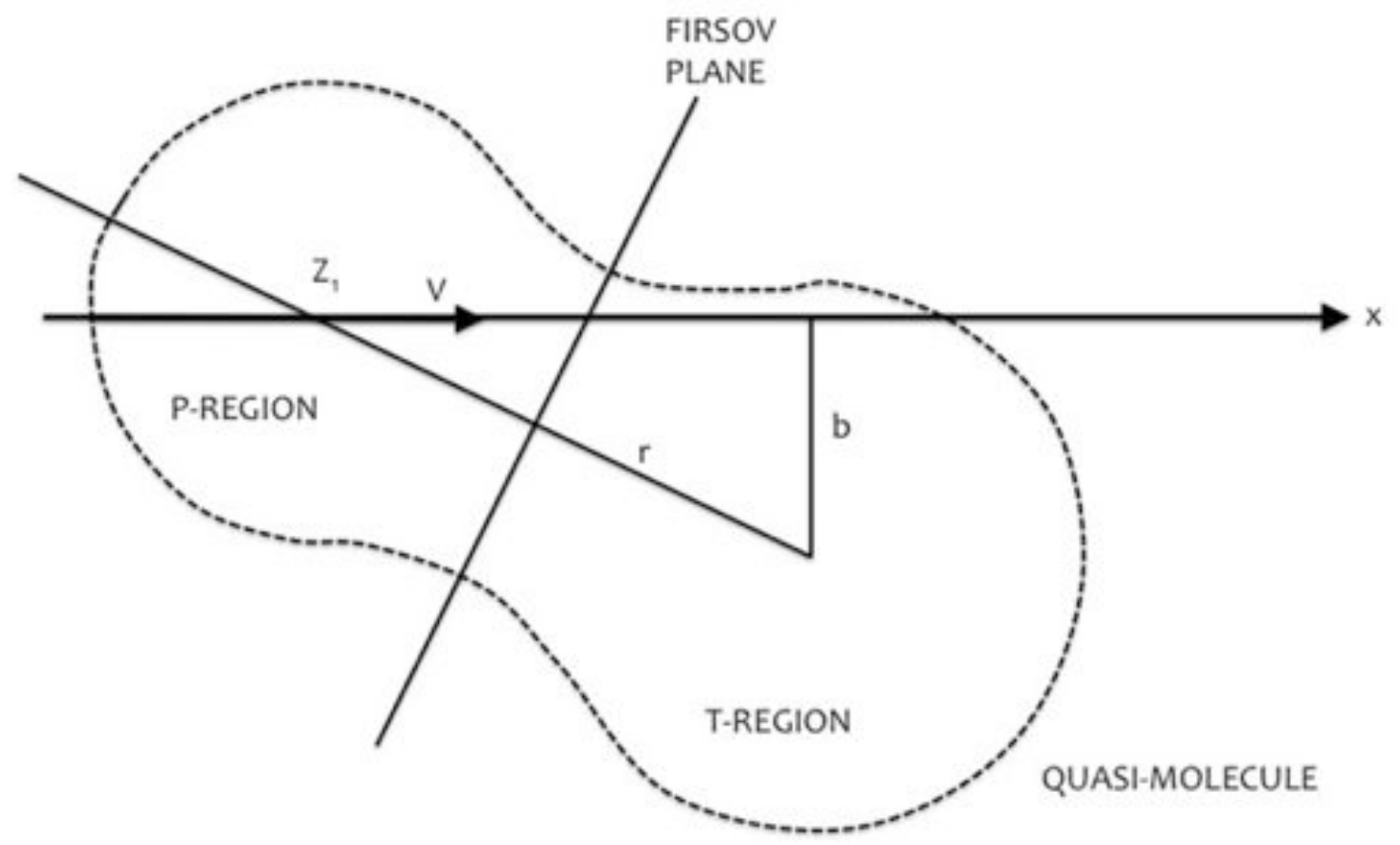

Figure 2.5: The Firsov quasi molecule, depicting the P-region, T-region and Firsov plane formed during electron stopping in the limit of $v<v_{0} Z^{2 / 3}$

\subsubsection{Nuclear stopping}

At low ion velocities, the collisions involve the projectile and the target atom as a whole, in comparison to high velocities where individual electrons are involved in the process. This type of collision is elastic in nature and can be described by two-body scattering, described previously. The energy transfer and deflection angles are large for this type of event, as the relative difference in mass of the projectile and the target atoms are much smaller than in the case of electron interactions. As the low energy projectile nears the target atom, the force experienced by the participants is that of a Coulomb interaction and can described by Coulomb potential scattering.

Due to the collision taking place between the projectile and the target atom as a whole and the large energy transfers, this type of interaction does often lead to atomic displacements. In order to displace a target atom, a minimum amount of energy must 
be transferred to the target atom to dislodge it from its current potential well. The energy necessary to make this occur is called the displacement energy. In typical solids, the solid-state configuration represents a local minima of the free energy of the system. Therefore, to displace an atom from its current location and allow it to become mobile requires enough energy to overcome the potential barrier. If a collision results in an amount of energy transfer that is less than the displacement energy, the collision will not result in the target atom becoming mobile. This energy imparted to the target will be dissipated in the form of lattice vibrations, or phonons.

During the nuclear stopping process, projectiles are able to dislodge target atoms from their present location and create a primary knock-on atomic (PKA). If the energy transfer is large, the PKA can continue the knock-on-atom process and induce further displacements. This process is commonly referred to as a displacement cascade. The displacement cascade (damage cascade) is thought to progress in a series of stages. The first is the collisional phase, where a PKA initiates a cascade of displacements until such time that there is no longer sufficient energy to produce subsequent displacements. Next, the thermal spike phase occurs in which the energy due to the collisions is shared amongst nearest neighbors. After the energy has been dissipated, the material is said to have undergone a quench, or reached thermodynamic equilibrium. The annealing stage is the final stage where defects, created by the damage cascade, find their final location.

\subsubsection{Primary knock-on atom displacements}

The model proposed by Kinchin and Pease [22], and expanded upon by others is often used to calculate the average number of displaced atoms in a sample during ion irradiation. There are, of course, more extravagant models such as the Norgett, Robinson and Torrens (NRT) model which account for inelastic energy loss using 
Lindhard's theory, but they will not be discussed herein.

The Kinchin-Pease model assumes a random target, the hard sphere approximation and stationary targets. The initial PKA is assumed to have energy $T$. The displacement energy, $E_{d}$, of the target takes a value which has been averaged over a range of displacement energies. If a target atom receives an energy $T>E_{d}$, the atom is liberated from its present location and in turn becomes a PKA, whereas if $T<E_{d}$, the atom remains in the present location. At energies between $E_{d}<T<2 E_{d}$, the collision process has only enough energy to liberate one atom before the energy is below the threshold energy of $E_{d}$. If nuclear stopping were the only mechanism, this would be the end of the model, where the average number of displacements would simply be incident energy divided by two times the displacement energy. However, electronic stopping must also be taken into consideration. For this reason, a cutoff value of $T=E_{c}$ is introduced such that no displacements can take place until the energy is lowered below $E_{c}$. For energies lying between $2 E_{d}<T<E_{c}$, the average number of displacements is just $T /\left(2 E_{d}\right)$. A detailed description of the Kinchin-Pease model is provided in Equation 2.9

$$
\text { average number of displacements }= \begin{cases}0, & T<E_{d} \\ 1, & E_{d}<T<2 E_{d} \\ \frac{T}{2 E_{d}}, & 2 E_{d}<T<E_{c} \\ \frac{E_{c}}{2 E_{d}}, & T \geq E_{c}\end{cases}
$$

If the energy deposition as a function of depth, $F_{d}(x)$, is known or tabulated, an approximation can be used to determine the number of displacements per atom (DPA), from the fluence $\left(\mathrm{cm}^{-2}\right)$. This relationship is given by, 
Rev. 1.2

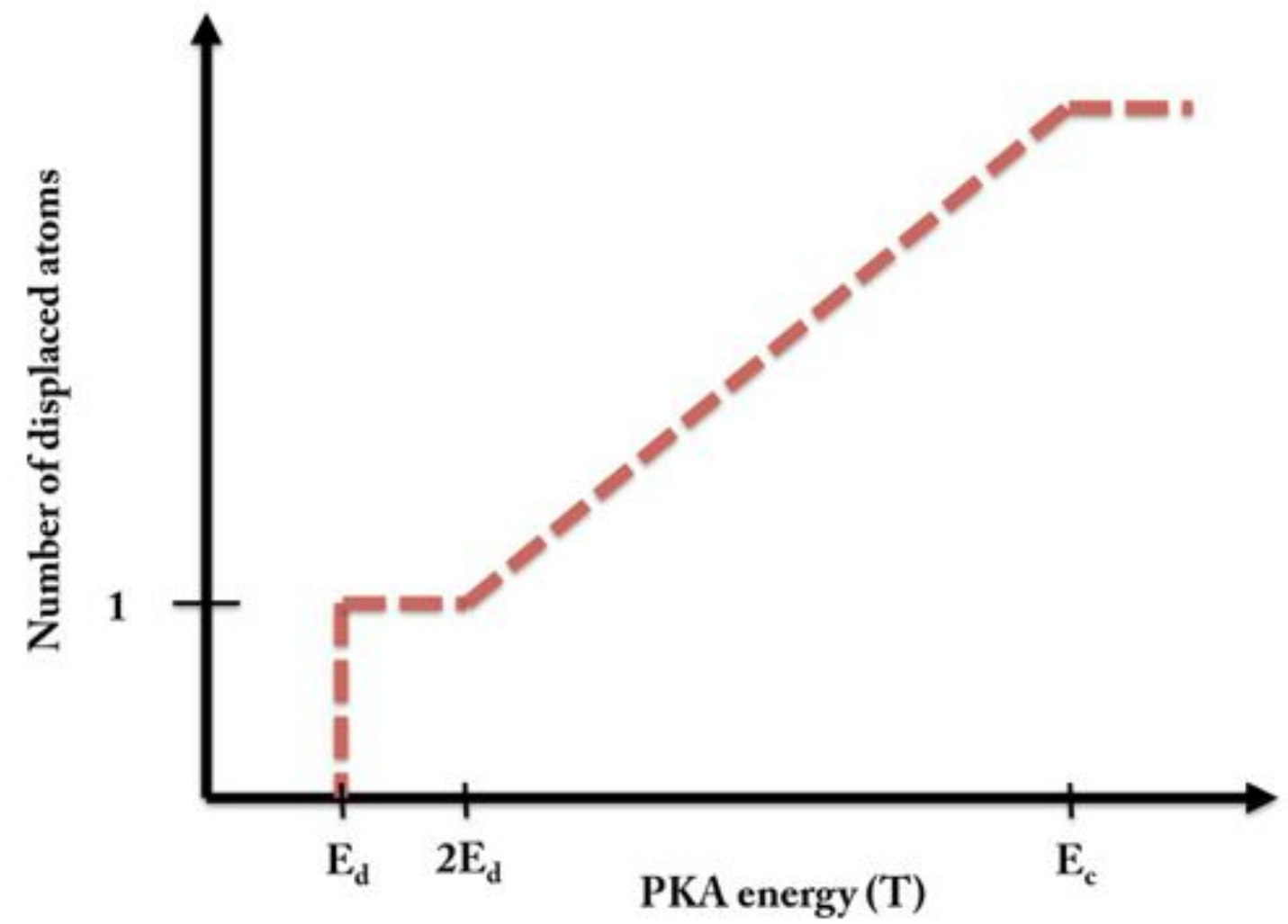

Figure 2.6: Graphical depiction of the Kinchin-Pease model which depicts the number of displacements based on the energy distribution function $\left(F_{d}\right)$, the displacement energy $\left(E_{d}\right)$, and the fluence $(\Phi)$.

$$
D P A(x)=.8 \frac{F_{d}(x)}{N \cdot 2 E_{d}} \Phi
$$

where $\Phi$ is the fluence, $F_{d}(x)$ is the energy distribution function, $N$ is the number of displacements and $E_{d}$ is the displacement energy. A graphical representation of this model can be seen in Figure 2.6.

In the Kinchin-Pease model, there is no absolute accounting for the spatial distribution of displacements during the damage cascade. Understanding the number of displaced atoms is important, but also being able to predict the relative proximity to one another is essential to understand radiation damage buildup. 


\subsubsection{Displacement mean free path}

The distribution of damaged regions caused by collisions is determined by the displacement mean free path. The mean free path is defined as the average distance traveled per interaction,

$$
\lambda=\frac{1}{N \sigma}
$$

where $N$ is the number density, $\sigma$ is the cross section. The cross section can be thought of as a likelihood for an interaction, and can be derived from quantum mechanics. The relationship between the cross section and the displacement cross section, $\sigma_{d}$ can be expressed by,

$$
\sigma_{d}(E)=\int_{E_{d}}^{E} \sigma\left(E^{\prime} \rightarrow E\right) d E^{\prime} .
$$

where $E_{d}$ is the displacement energy, $E$ is the final energy and $E^{\prime}$ is the initial energy. Therefore, this represents the cross section for transferring energies in excess of the displacement energy. Using the hard sphere approximation and the Born-Mayer potential to evaluate $\sigma$ gives

$$
\sigma_{d}(E)=\pi B^{2}\left[\ln \left(\frac{2 A}{E}\right)\right]^{2}\left(1-\frac{E_{d}}{E}\right)
$$

where again, the constants $\mathrm{A}$ and $\mathrm{B}$ are determined experimentally and the mean displacement mean free path, $\lambda_{d}$ becomes

$$
\lambda_{d}=\frac{1}{N \pi B^{2}\left[\ln \left(\frac{2 A}{E}\right)\right]^{2}\left(1-\frac{E_{d}}{E}\right)} .
$$

At large recoil atom energies, the mean free path is large and therefore the dis- 
tance between successive collisions is well separated. As the energy of the projectile decreases, the spacing between collisions approaches the order of atomic spacing. At this point, each atom along the path is displaced. The damage can no longer be thought of as a collection of point defects and instead leaves behind a highly damaged volume consisting of a shell of interstitials and a core of vacancies [23]. This leads to the notion of a displacement spike. An illustration of a displacement spike can be seen in Figure 2.8 which shows that an incoming ion creates a PKA, which in turn, creates a displacement spike with a core of vacancies and a shell of interstitials (originally proposed by Brinkman [23]).

\subsubsection{Thermal spike}

At the end of the displacement spike, a highly localized region has experienced a large increase in energy in a very short period of time. In typical two-body collisions, the energy transfer can be solved analytically, but in the case of many-body collisions, as in the case of a recoil coming to rest, the problem is not so simple. The energy due to the collisions is transferred between many atoms and the result is a net increase in temperature around that region shared amongst nearest neighbors that does not result in displacement creation. The heat spike, therefore, is mainly attributed to energy dissipation of collision processes which do not result in displacements. The time-scales over which these high temperatures persist are on the order of picoseconds and quickly cool to ambient temperatures. A simple correlation has been suggested [16] to place a lifetime on the thermal spike induced by a damage cascade, given by

$$
t=r^{2} / 4 D_{t}
$$

where $t$ is the lifetime of the thermal spike, $r$ is the radius over which the energy is deposited and $D_{t}$ is the thermal diffusivity. 


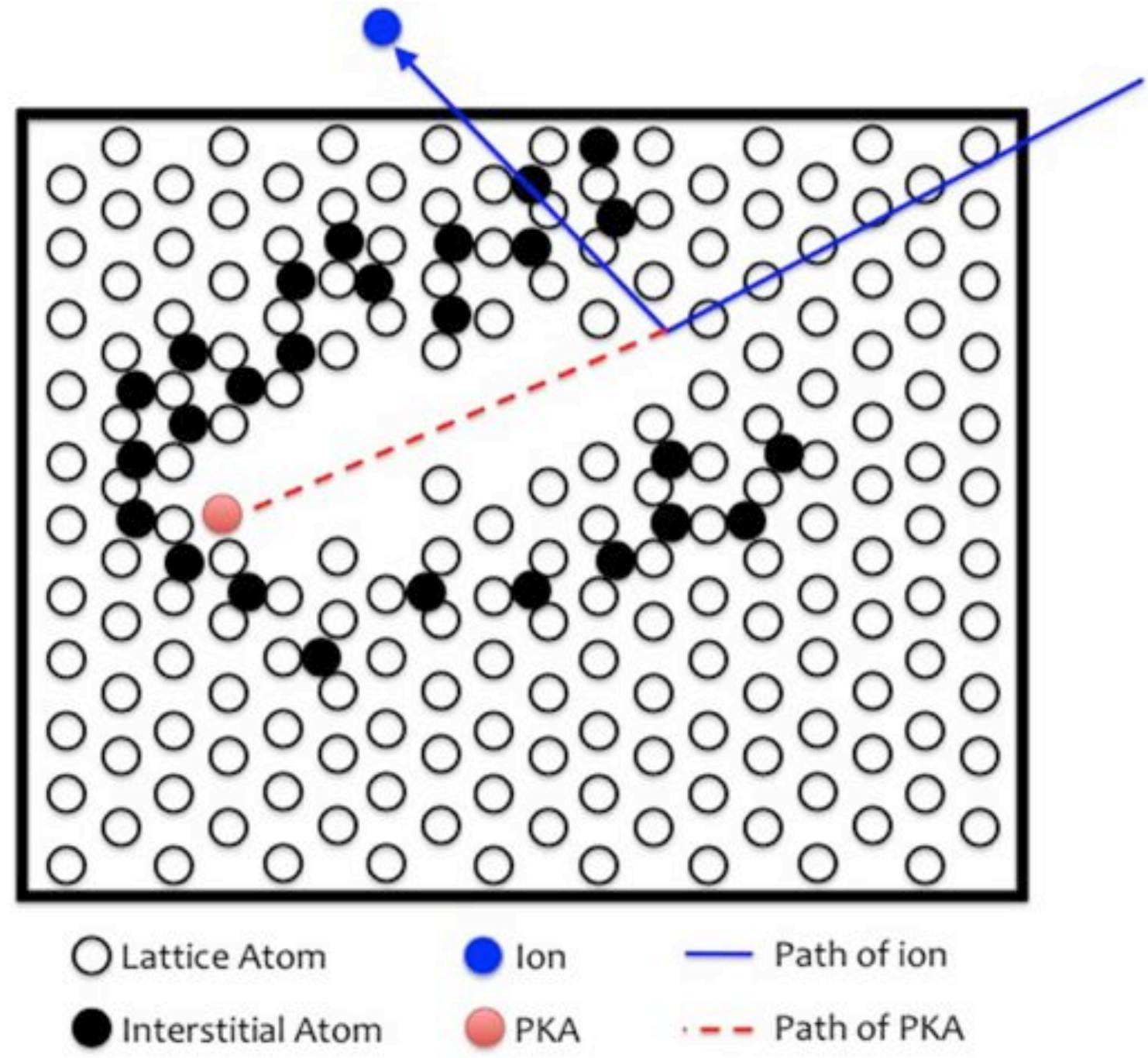

Figure 2.7: Illustration of the displacement spike as a result of collisions occurring with a displacement mean free path on the order of the average atomic spacing. 
During this time, defects are able to migrate and recombine, resulting in a reduced number of defects compared to the initial number ballistically generated in the damage cascade [19]. Once a thermodynamic equilibrium has been established, the quench has considered to have occurred. Final annealing occurs due to thermally activated diffusion and lasts until all the available defects that are able to migrate have done so. This implies that the time for this stage to come to completion is highly dependent on the temperature and irradiation conditions and occurs over a wide range of time-scales.

\subsubsection{Dynamic annealing}

As stated above, an energetic ion propagating through a solid creates a collision cascade along its trajectory. The ballistic formation and thermalization of the cascade occur rapidly, at time-scales of up to $\sim 10^{-12} \mathrm{~s}$. This ballistic stage of defect production is considered to be well understood (excluding cases when cascades are non-linear) $[22,24,25]$. In contrast, our current understanding of the evolution of defects after cascade thermalization, which is often referred to as dynamic annealing (DA), is limited for most materials [26-28].

Understanding mechanisms involved in DA is, however, highly desirable since DA plays a major role in the formation of stable post-irradiation disorder in most technologically relevant cases, including ion-beam-processing of semiconductors and radiation damage in nuclear materials [24, 27-32]. It is the DA that, in most practical cases, largely determines the form and extent of stable lattice damage in solids after irradiation and materials" "radiation-tolerance" [22, 24, 25].

The time scale of DA processes; i.e., a characteristic time constant $\tau$ over which the dominant processes of defect evolution persist after the thermalization of collision cascades is determined by the thermal stability, effective diffusivity, and specific 
Rev. 1.2

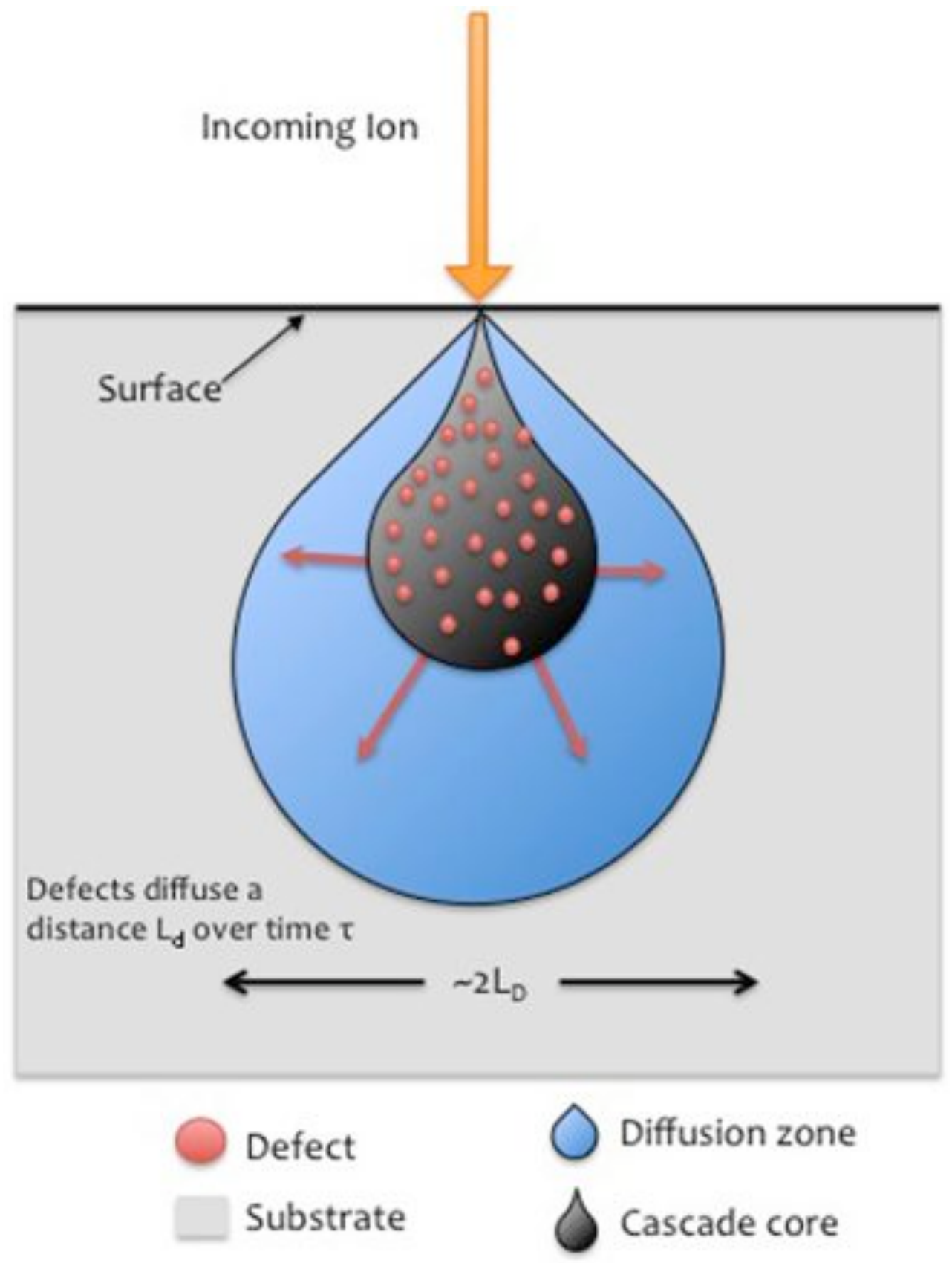

Figure 2.8: A schematic showing the parameters associated with DA for an individual ion impinging on a sample surface. 
interaction processes of radiation-generated defects. Figure 2.8 schematically depicts the DA process for an individual damage cascade. Initially, an ion strikes a sample surface which creates a damaged region below, shown as the black zone (in Fig. 2.8). However, a short time later, defects can diffuse radially outward (the blue area in Fig. 2.8) a distance $L_{\mathrm{d}}$ over a characteristic time $\tau$. Knowledge of $L_{\mathrm{d}}$ and $\tau$ are important for the development of physically sound models of damage accumulation in solids in order to control and fully exploit the effects of radiation damage.

\subsection{Fluence effect}

During ion irradiation, ion-solid interactions induce structural transformations. This is due to energy being ballistically transferred from impinging ions to target atoms and recoils [16]. With increasing fluence, disordering increases, and amorphization can occur. A damage cascade overlap model has been used to describe the fluence-effect driven amorphization process [33,34]. Isolated amorphous zones occur along ion tracks (shown in Fig. 2.9), and upon continued bombardment, partially damaged zones begin to overlap. In Fig. 2.9, an incoming ion strikes in the vicinity of a previously damaged region, resulting in the formation of permanent damage in the overlapped region.

The fluence effect has been shown to be highly non-linear [35]. In the low fluence regime, pre-existing defect structures act as undersaturated defect sinks, readily annihilating Frenkel pairs. This results in sub-linear behavior [36]. After exceeding a threshold fluence, a rapid super-linear increase in disorder is observed. The super-linearity has been attributed to a reduction in the threshold energy for atomic displacements in a pre-damaged crystal. Therefore, the concentration of stable defects is proportional to the concentration of existing defects, a so-called sensitization process [36]. 
Rev. 1.2

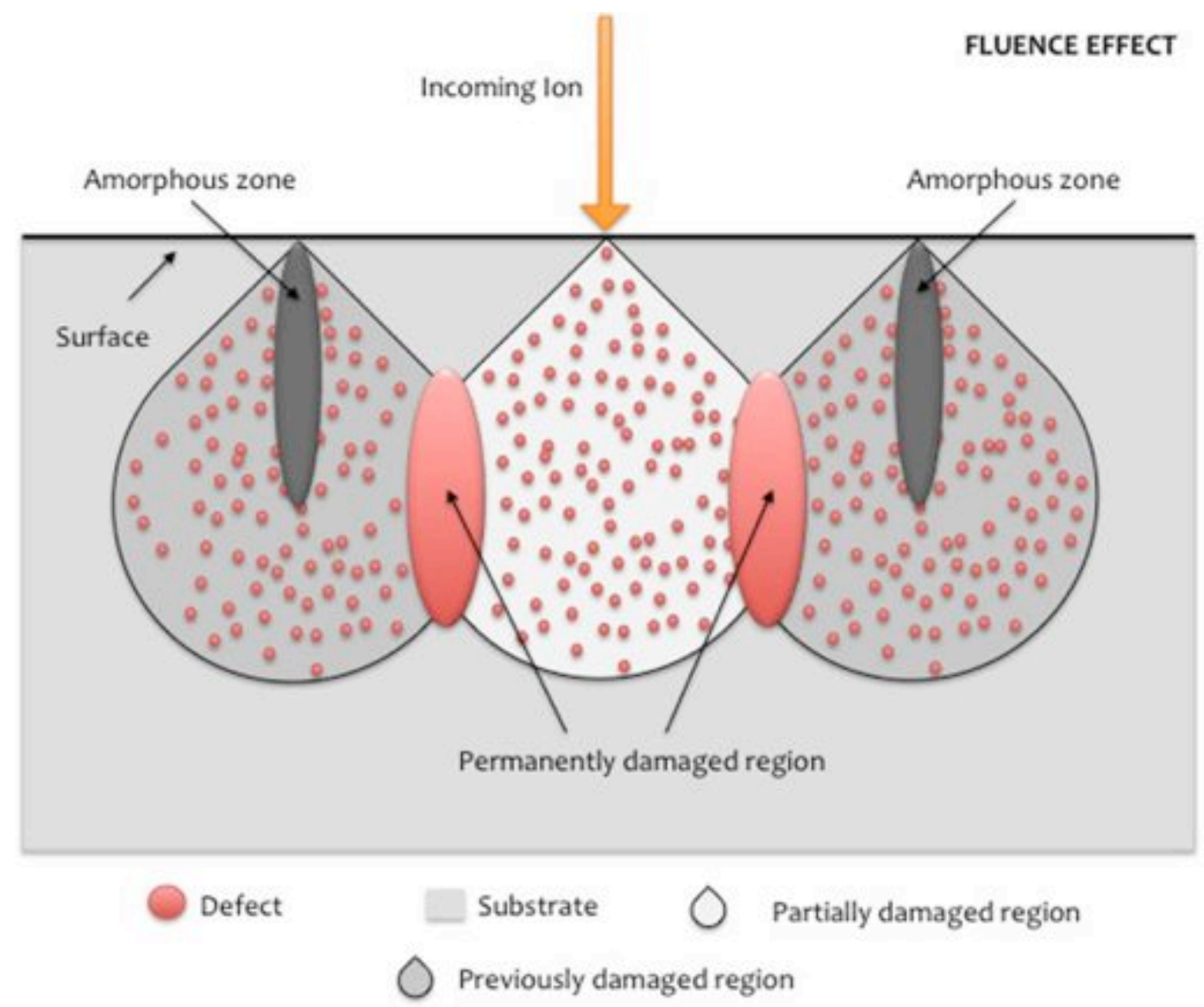

Figure 2.9: A schematic of the fluence effect showing increased defect accumulation in overlapping damaged regions. 
Rev. 1.2

\subsection{Molecular effect}

A similar type of non-additive damage accumulation has also been observed in the molecular effect (ME). That is, polyatomic ions produce more damage than the same ions implanted separately [37]. The explanation of ME for heavy ions is based on differences in energy density between the ions impinging independently versus molecularly. A damage cascade overlap model put forth by Crowder and Morehead [33] and later expanded upon by Gibbons [34] seems to provide a reasonable explanation for the effect. In fact, depth dependent calculations on the spatial separation of sub-cascades during heavy ion irradiation leads to ME occurring only near the surface, where the cascades are highly overlapped [38]. In contrast, ME during light ion irradiation has been shown to be strongly correlated to the ion beam flux [39], where the efficiency of ME decreases with increasing ion flux. That study [39] also suggested that DA and the density of collision cascades both play a vital role in understanding the molecular effect.

\subsection{Flux effect}

The flux effect is a dependence on the amount of stable lattice disorder on the incident ion flux, with all other parameters held constant. A flux effect will be prevalent when the vacancy concentration outside the collision cascade track is on the same order of magnitude as the vacancy concentration in the bulk [33]. Fig. 2.10 shows that, if nearly simultaneous ions arrive at the sample surface in near proximity to one another, the regions over which defects can normally diffuse after cascade thermalization begin to overlap. Such overlapping results in limited defect diffusion due to the similarity of vacancy concentrations inside and outside the cascade core (shown as blue arrows in Fig. 2.10).

In this case, there should exist two distinct time-scales related to the flux effect, 
one that is independent of temperature [the initial cascade collapse $\left(<10^{-12} \mathrm{~s}\right)$ ] and another that depends strongly on temperature and occurs over much longer timescales [33]. Carter et. al proposed that the nature of the defects not only depends on the fluence, but also directly on cascade stabilization times [40-42].

The defect kinetics and the associated time constants will play a large role in the manifestation of the flux effect [43]. The flux effect is rarely observed at low fluence [44]. This is due to the nature of various dynamic defect annealing phenomena that are active at RT. However, with increasing fluence, the flux effect is more pronounced [43]. Stable defect complexes form, and are less susceptible to annihilation and diffusion. For very high flux values, experimentally measured damage profiles start to approach those predicted using BCA models, such as TRIM [20]. This is even more evident at low temperatures where defect diffusion is even more limited. However, in some cases, disorder levels have been observed to decrease with increasing fluence at very high flux values [42]. One explanation for this is thermal annealing. Ion beam power deposition would lead to enhanced defect recombination at higher flux values due to beam heating $[42,43]$.

In contrast to this, an inverse flux effect has been observed at very low fluence. To explain this, Svensson et. al suggested that high flux values result in rapidly expanding self-interstitials that would overlap and annihilate vacancies created in adjacent damaged zones [44]. A reverse temperature dependence on damage buildup has also been observed which has been attributed to a more stable distribution of simple defect structures at low temperature. This acts to confine vacancies to a smaller volume for longer durations leading to increased annihilation from adjacent ion tracks [44]. Clearly, for both the flux effect and the inverse flux effect, the defect interaction times, the cascade stabilization time, and the lengths over which these processes occur are of fundamental importance. 


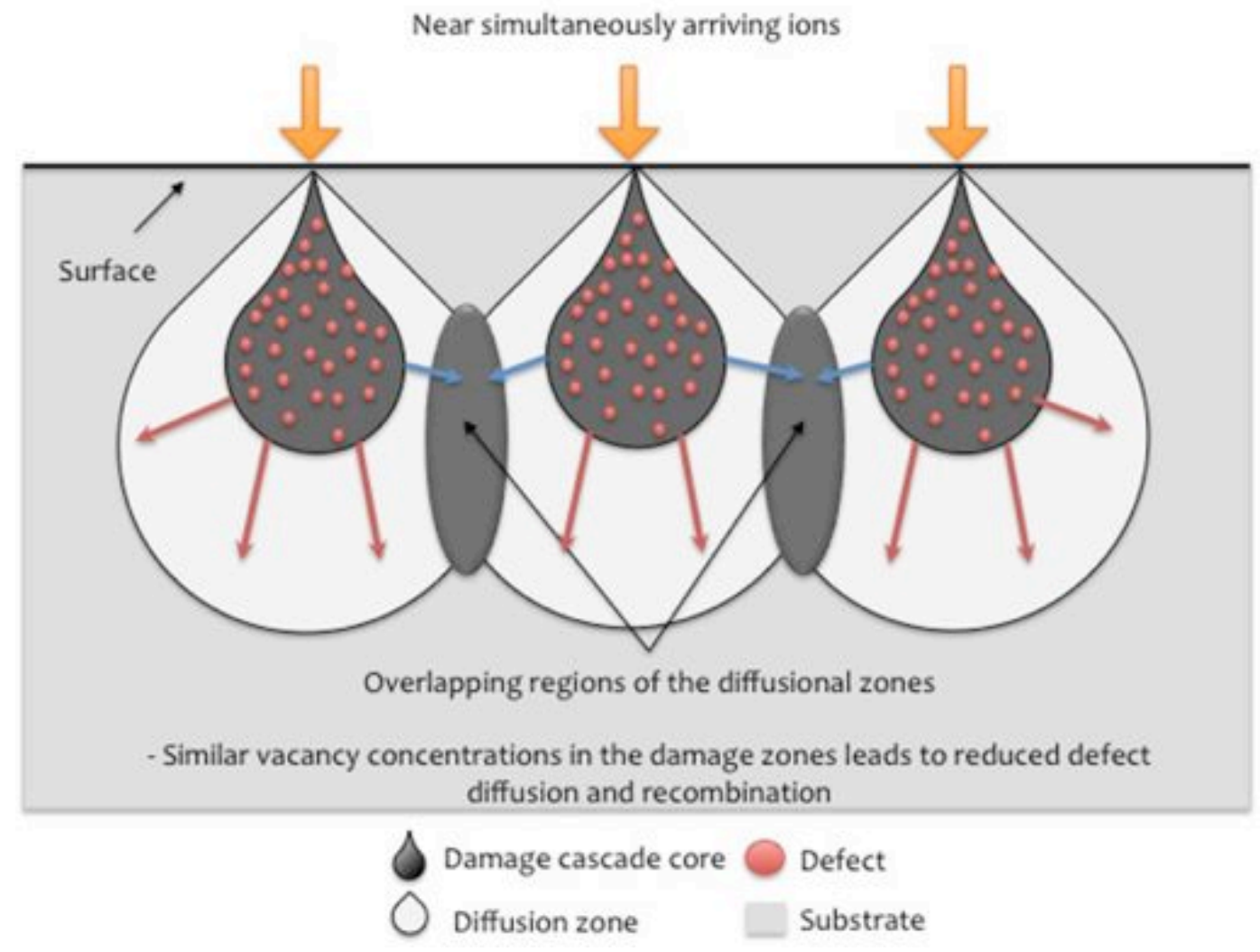

Figure 2.10: A schematic of the flux effect showing increased defect accumulation due to similar vacancy concentrations in damaged regions within the diffusional zone. 
Rev. 1.2

\section{CHARACTERIZATION METHODS}

The present section provides a brief introduction into the two main characterization methods which were employed in this dissertation, high energy ion scattering (HEIS) and transmission electron microscopy (TEM). In the following sections (4 and 5), results from ion beam analysis (IBA) and TEM are routinely discussed, warranting a introduction to the methods which were used.

\subsection{High energy ion scattering and channeling}

Rutherford backscattering spectrometry (RBS) is one of the most widely used IBA techniques. The process of HEIS involves the measurement of the quantity and energy spectrum of light ions, typically $2 \mathrm{MeV} \mathrm{He}$, which are backscattered from a sample into a detector [17]. This situation is shown in Fig. 3.1(a) with two detectors. The shallow glancing angle detector is used to resolve near surface features (up to a few hundred nm, depending on angle), whereas the backscatter detector is used to resolve features that occur further from the sample surface into the bulk (up to a tens of microns).

Assuming a mono-energetic beam of ions, energy loss will be due to two major components. The first is electronic stopping, which, assuming that the stopping power does not vary across the thickness of the sample, is directly proportional to the ion energy in that layer. The second component to the stopping is nuclear energy loss due to a classical two body collision with a target atom, which can be treated with classical scattering theory (described in Section 2). Along certain incident ion directions, the amount of particles which are backscattered into the detector reaches a minimum value. This is due to the phenomena of ion channeling, which can been described in terms of Linhard's theory [45] on the topic. Figure 3.1(b) shows the 
Rev. 1.2

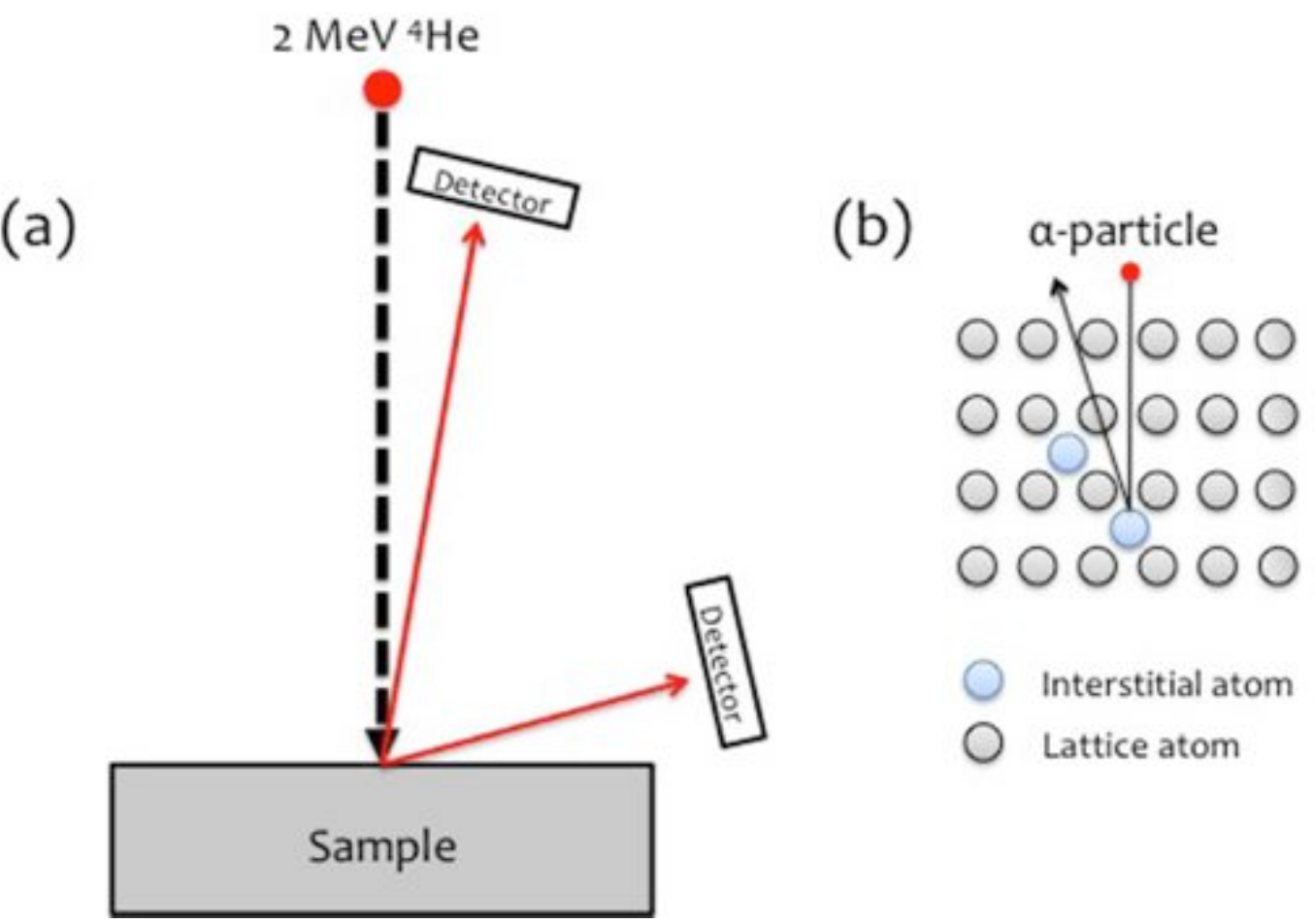

Figure 3.1: Typical arrangement for Rutherford backscattering spectrometry performed in the channeling orientation. (a) Illustrates the use of both a glancing detector for enhanced near surface resolution (typically $\lesssim 15^{\circ}$ ), and a backscatter detector which provides enhanced depth resolution (typically $\sim 164^{\circ}$ ). (b) Depicts an $\alpha$ particle (i.e. a He atom) scattering off of an atom that lies in the channel (interstitial).

scattering of $\mathrm{He}^{+}$probing particles from atoms which are situated in crystallographic channels [shown as interstitials in Fig. 3.1(b)]. This phenomena leads to an increase in the number of particles which are backscattered into the detector. The energy deposited in the detector from the backscattered particle corresponds to the depth at which the scattering event took place, after accounting for the electronic energy loss along both the incoming and outgoing paths of the probing ion. The resultant spectrum is a depth profile of disorder with a superimposed spectrum from the dechanneling component (described below).

In order to perform ion channeling on a sample to determine the distribution of 
atoms which have been displaced from their lattice positions, the sample must be precisely oriented using goniometers. The motors on the goniometer typically move in increments of $.005^{\circ}$ to allow for precise determination of the minimum channeling yield. One popular method for finding the minimum of the channeled yield is performed by systematically tilting the sample. Backscattered yields are recorded by fixing the overall tilt range to some value, i.e. $\pm 5^{\circ}$, and continuously tilting along the other direction to $\pm 5^{\circ}$, in small steps, until the minimum is obtained. Once is it obtained, typically, slight adjustments are made to find the absolute minimum backscattered yield. If the sample is not aligned, the channeled spectrum may not reach a minimum and give a spurious signal when trying to calculate damage-depth profiles.

The channel numbers in the backscattered spectrum are directly related to the energy of the particles which have been scattered into the detector. However, the absolute channel number associated with a particular energy is a function of many parameters, including the detector bias, preamplifier setting, main amplifier, analogto-digital convertor, and beam-sample-detector configuration. In order to simulate the spectrum (using software such as RUMP), typically a calibration sample in the form of a thin film grown on a substrate is required. This allows for an accurate determination and conversion of channels to energy. For very thin samples, the channel number will correspond to the peak of a delta like function. Given that the calibration consists of a sample of known composition, the energy of a backscattered particle is easily calculable and therefore, the energy of the channel is known. This value is typically referred to as a kinematical factor and are tabulated for a number ion-target combinations as a function of energy and detector angle $[17,18]$. If there are two standards (of different mass) from which to calculate energy transfer, a linear relationship exists between energy and channels (i.e. $\left.\frac{\Delta E}{\Delta n}\right)$. 
With the assumption that that layer is thin, such that the stopping power is constant across the layer, the energy can be converted to depth in the substrate by using the following equation,

$$
E(t)=k\left[E_{0}-\frac{t}{\cos \phi_{1}}\left(\frac{d E}{d x}\right)_{E_{0}}\right]-\frac{t}{\cos \phi_{2}}\left(\frac{d E}{d x}\right)_{E}
$$

where $\mathrm{E}(\mathrm{t})$ is the energy recorded by a detector for particles backscattered at the depth $t, \phi_{1}$ is the incident beam angle, $\phi_{2}$ is the exit angle, $\left(\frac{d E}{d x}\right)_{E_{0}}$ is the energy loss of the ion while penetrating into the sample and $\left(\frac{d E}{d x}\right)_{E}$ is the energy loss while traversing out from the sample toward the detector.

For most ion-target combinations which consist of monoatomic samples, the stopping powers are widely known, or tabulated, and can be obtained using the TRIM code [20]. For compound targets, less information is generally available, however a good approximation is to use a weighted mass and nuclear charge. This provides accurate results so long as the constituents do not vary in mass or charge substantially.

A typical RBS/C spectrum has components that arise from two phenomena. The first contribution is from particles which are backscattered into the detector. The second contribution is from particles which have been dechanneled as they traverse the depth of the material. In order to extract useful information on the depth profile of displacements (lattice interstitials), the dechanneling component must be subtracted from the spectrum.

There are at least three methods for performing a dechanneling calculation. The one used in the present work is that of the single iterative method. In this method, the substrate is divided into thin layers and the calculation typically proceeds by a proper choice of the sample surface and the depth at which the bulk defect peak ends. 
Within the first layer, the backscattered yield is due to displacements only and can be directly converted to displacements. In the next layer, the backscattered yield is due to both dechanneling that occurs in the first layer and displacements in the second layer. A subtraction is made for the dechanneling component in this layer due to the previous layer. This processes is repeated until the entire thickness of the substrate has been performed and the entire dechanneling component has been obtained.

At this point the spectrum has been converted to relative disorder, which is defined as the normalized concentration of defects divided by the atomic concentration of the sample (in atoms/cc). Relative disorder is unitless, however, it is typically expressed in terms of percent, with $100 \%$ referring to a fully amorphous structure and $0 \%$ referring to a perfect crystal. For more details pertaining to this calculation, see Appendix A and Ref. [17].

Ion channeling can be used in the determination of crystal orientation, lattice imperfections, impurity location, and the effects of radiation damage. A more detailed description of RBS/C techniques can be found in Ref. [17].

\subsection{Transmission electron microscopy}

In a conventional microscope, light is used to image a surface and the resolution is limited by the wavelength of light. In order to overcome this hurdle and resolve images over a thousand times smaller than a light microscope, electrons are used. The small wavelength of an electron enables imaging on an atomic scale. Transmission electron microscopy has become the quintessential tool for materials characterization. It is a destructive method, whereby samples are first prepared using either a conventional thinning method or a focused ion beam (FIB) and then analyzed under the microscope. A typical TEM consists of an electron gun, which provides the 
source, followed by a series of condenser lenses and an objective lens that illuminates an electron beam onto an electron transparent specimen. The electron source is comprised of an electrode and a cathode, which, when heated emits electrons. The potential between the anode and cathode accelerates the electrons toward the anode. A fraction of the electrons pass through a central hole in the anode and emerge as a loosely focused beam of electrons. A series of apertures and lenses are then used to produce a monochromatic, collimated and focused beam. After passing through the specimen, the transmitted electrons pass through the objective aperture and onto a CCD camera. The lenses located after the beam has passed through the sample are used to refocus and enlarge the image. An illustration of this process is found in Figure 3.2.

The two basic modes of operation for a TEM are the diffraction and imaging modes. By making an adjustment to the focal length within the intermediate lens, operation can be switched back and forth between the two modes. A diffraction pattern is always present in the back focal plane of the objective lens. Contrast is increased by inserting an objective aperture at the back focal plane of the objective lens. Bright field (BF) TEM consists of using a larger fraction of the direct beam to image the specimen, whereas Dark Field (DF) uses the forward scattered, deflected electrons by moving the objective aperture to block the central beam of unscattered electrons.

Diffracted electrons which pass through the specimen can be used to create diffraction patterns. The diffraction pattern is imaged and indexed using the relationship,

$$
d=\frac{\lambda L}{R}
$$

where $d$ represents the lattice spacing, $R$ the distance from the central Bragg peak 
Rev. 1.2

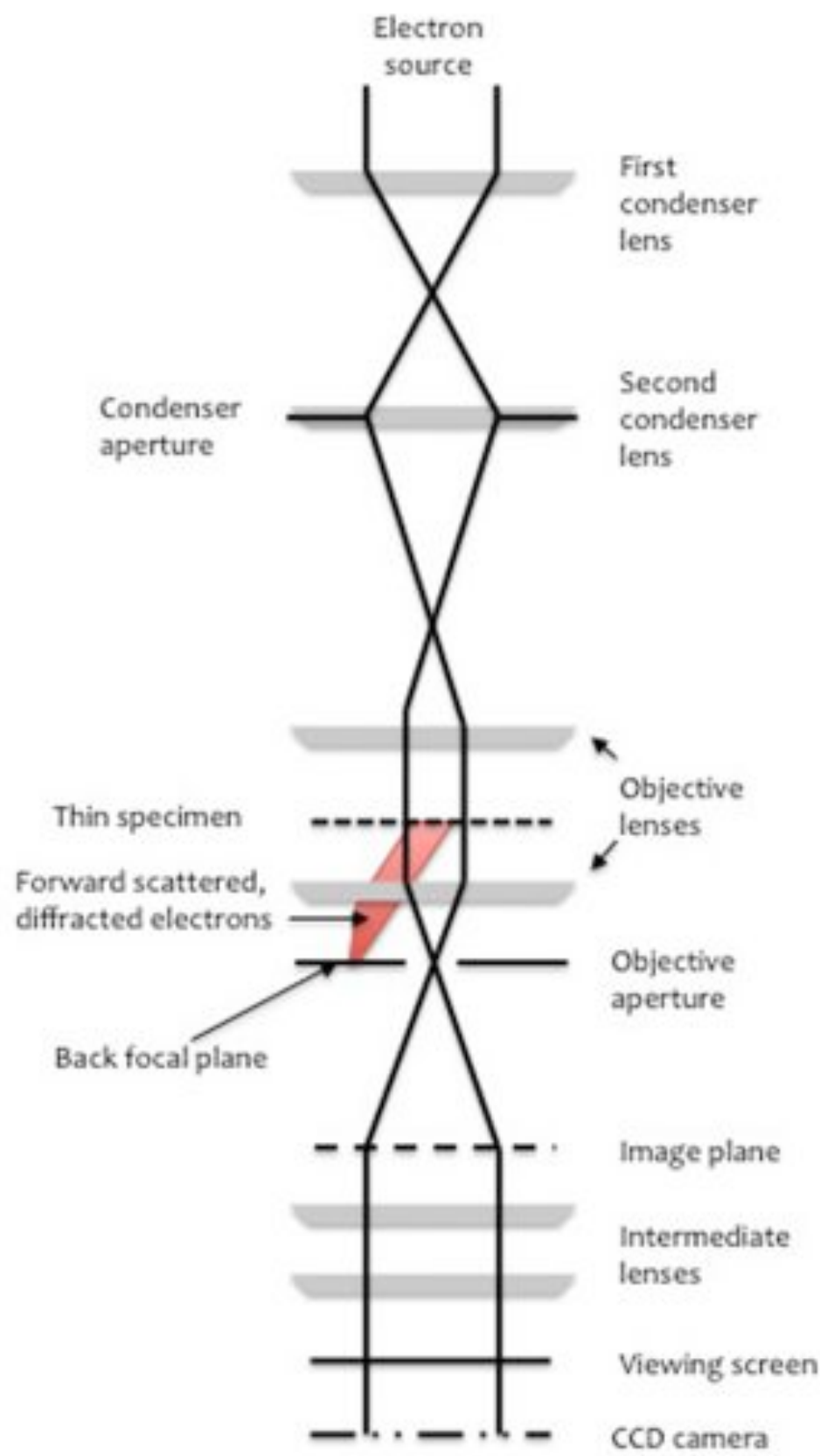

Figure 3.2: Illustration of the working principles and components of a transmission electron microscope 
to the radial peak of intensity (usally a ring, or point), $L$ is the camera length and $\lambda$ is the electron wavelength.

In addition to conventional TEM, Scanning TEM (STEM) employs the same conceptual basis as TEM, except STEM is able to scan a line of interest and determine atomic composition using the principle of energy dispersive X-ray spectroscopy (EDS). The same technology can be used in TEM, but the difference is that, only a single point in a standard TEM is chosen for analysis, whereas in STEM, there is a line which is scanned. As electrons penetrate the sample, inelastic scattering events will take place that excite target electrons in the material to higher orbits. These excited electrons then de-excite through the emission of characteristic X-rays. The frequency and intensity of these x-rays is then measured to provide insight into the chemical composition and forms the basis for EDS analysis.

A more thorough treatment of the aforementioned topics can be found in Reference [46, 47], including diagrams, operational procedures and a more detailed description of the theoretical basis for electron microscopy. 
Rev. 1.2

\section{HEAVY-ION-BOMBARDED ZINC OXIDE}

The present section consists of an introduction to radiation damage in ceramics, a description of $\mathrm{ZnO}$, a background on radiation damage studies in $\mathrm{ZnO}$, followed by our reports on a series of interrelated experiments in $\mathrm{ZnO}$. The experiments were performed to elucidate the mechanisms of an IP that has been observed in ion channeling spectra in heavy-ion-irradiated $\mathrm{ZnO}$. The first experiment focuses on the role of the surface by precisely controlling the depth of the displacement generation profile. Next, a detailed microstructural analysis using RBS/C, cross sectional TEM (XTEM) and STEM was performed to correlate the microstructure to ion channeling spectra. Then, a method to reduce IP formation (and damage in general) in $\mathrm{ZnO}$ by using surface modification is explored. Differences in DA are also investigated among the various surfaces (both polar and non-polar), further clarifying the role of the free surface on IP formation in (0001) ZnO. Finally, surface modification is applied to both polar and non-polar surfaces to determine if the dramatic effect observed in polar surface modified $\mathrm{ZnO}$ is present in non-polar surfaces.

\subsection{Introduction to radiation damage in ceramics}

Understanding the radiation response of ceramics has been driven mainly by two major groups: the semiconductor industry with ion implantation doping and isolation $[25,36]$, and the nuclear materials community [48-51]. First, radiation damage is the main limiting factor for the development of ion-beam processing techniques for the fabrication of oxide-based (opto)electronic devices [24, 52]. Second, there is a need to extend radiation tolerance of both structural and fuel materials for nuclear power reactors [31]. Despite decades of research, however, radiation defects in ceramics are incredibly complex, and thus, remain poorly understood [24, 32, 52, 53]. 
Ceramics consist of a broad class of materials whose bonding ranges from purely ionic, to purely covalent and whose electrical properties range from insulators, to semiconductors to superconductors [53]. Ceramics are typically more brittle than metals with substantially lower thermal diffusivity. The radiation response of ceramics depends largely on the structure, bonding and composition of the material. Due to the presence of multiple sub-lattices (in compound ceramics), radiation effects in these materials are considerably more complicated than their metallic counterparts $[53]$.

Ceramics can generally divided into two main groups: amorphizable and nonamorphizable. Defect accumulation is a secondary effect to the initial primary displacements during irradiation. One response that a material can exhibit during defect accumulation is the complete loss of long range order, otherwise known as amorphization. This occurs as a result of initial defects losing their individual identity and the solid adopting a uniformly defective state. The degree to which a ceramic is susceptible to the amorphization process is largely determined by the type of bonding [32]. Ceramics which typically exhibit a large degree of ionicity and/or metallic bonding, traditionally do not easily amorphize under irradiation without substantial chemical effects (i.e. $\mathrm{ZnO}, \mathrm{MgO}, \mathrm{MgAl}_{2} \mathrm{O}_{4}, \mathrm{UO}_{2}$ to name a few). On the other hand, materials with a high degree of covalent bonding, or mixed ionic-covalent have been found to be more easily amorphized (i.e. Si, $\mathrm{GaN}, \mathrm{Al}_{2} \mathrm{O}_{3}$ ).

The degree to which the crystalline structure can be recovered due to thermal annealing is also determined largely by the bonding type. For those materials which amorphize, the regrowth of the crystalline phase under annealing conditions can be easily explained by solid phase epitaxial recrystallization (SPER). However, for nonamorphizable systems, lattice recovery using thermal annealing can be quite complex and can display multiple stages. These stages are determined by the activation energy 
of migration for particular defect species (vacancies and interstitials). For a good review of damage recovery by annealing in crystalline oxides refer to Ref. [54].

Compounding the situation with ceramics, microstructural evolution during irradiation has been shown to be highly dependent upon the spectrum of the incident irradiation [55]. For example, it has been shown that the dose and temperatures required to amorphize alumina $\left(\mathrm{Al}_{2} \mathrm{O}_{3}\right)$, magnesium aluminate spinel $\left(\mathrm{MgAl}_{2} \mathrm{O}_{4}\right)$, magnesia $(\mathrm{MgO})$, silicon nitride $\left(\mathrm{Si}_{3} \mathrm{~N}_{4}\right)$ and silicon carbide $(\mathrm{SiC})$ is highly dependent on the ion mass [56]. This makes direct comparisons amongst various materials irradiated under different conditions exceedingly difficult, if not impossible, to apply to other systems.

One explanation for the observed irradiation spectrum dependence was described by Bourgoin and Corbett as ionization enhanced diffusion (IED) [57, 58]. It has been suggested that ionized point defects in semiconductors and insulators may have a lower migration barrier than that of non-ionized defects. The large ionization per unit damage associated with light ions may act to inhibit defect cluster nucleation [59]. Several studies have shown that ionizing radiation, using electrons, can promote the recovery of isolated amorphous regions in defective crystals [60] which further supports the concept of IED. The knock-on energy of an electron is far below the displacement threshold, therefore, this effect is solely dominated by ionization.

In highly ionic systems, defect creation can result in the production of charged defects (both point and extended) due to conservation of charge neutrality. Diffusion of charged defects through the lattice will largely depend on the surrounding charge configuration in the anion and cation sublattices. Clearly, ionization can have a large role in defect evolution in ionic systems, both in the initial defect creation and subsequent diffusion. Additionally, many ionic systems contain polar surfaces with an intrinsic electric field due to a lack of an inversion center in their crystal 
Rev. 1.2

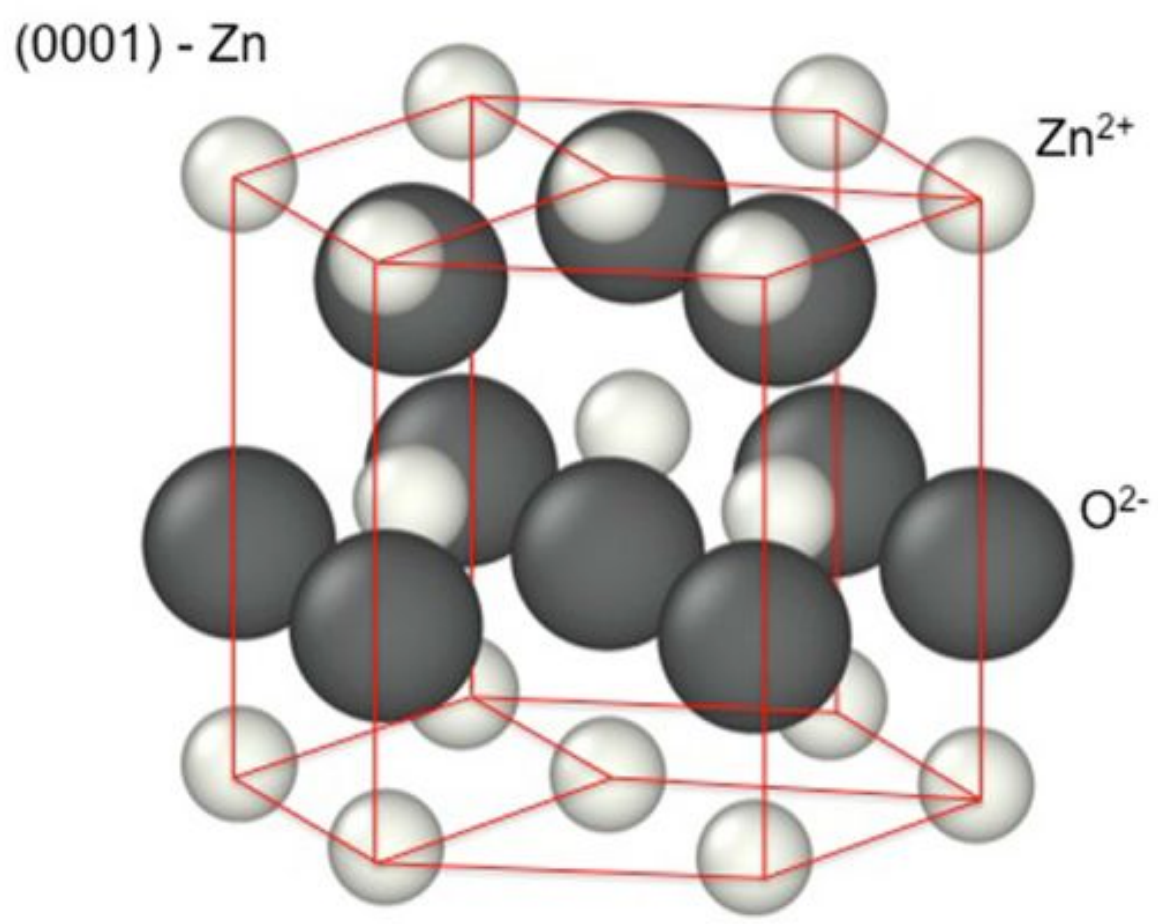

Figure 4.1: Wurtzite crystal structure for ZnO. Dark grey spheres represent oxygen atoms and light grey spheres represent zinc atoms. The polar zinc terminated face (0001) is at the top of the figure.

symmetry [61]. Cleaving these crystals along certain orientations will result in an excess charge on the free surface. This results in a spontaneous polarization along well defined crystal axes, typically referred to as polar axes. Polarity compensation can result in complex surface behavior, such as partially filling of the electronic states and adsorption of foreign species. In semiconductor materials, polarization effects have profound implications due to the quantum confined Stark effect which can substantially reduce device performance. Reference [61] contains a review of the classification of the main low index crystal surface terminations for most prototypical structures of oxides.

One particular class of ceramic that has garnered significant attention in the past two decades, with respect to radiation damage in particular, are the compound 
semiconductors in the II-VI and III-V families. Two such examples are $\mathrm{ZnO}$ and GaN which are direct, wide band gap semiconductors with a wurtzite crystal structure ( $\mathrm{ZnO}$ shown in Fig. 4.1). The lack of an inversion center in the wurtzite structure results in the formation of (major) polar surfaces along the c-axis (0001) and is a class III polar surface according to Tasker's classification [61]. The three major surface terminations, corresponding to unreconstructed $\mathrm{ZnO}$, are shown in Figure 4.2, with $C$-plane being polar, and $A$ - and $M$-plane being non-polar.

Currently, GaN substantially overshadows $\mathrm{ZnO}$ as the material of choice for most semiconductor optoelectronic devices (i.e. Bluray) due to the ability to tune the doping levels to stimulate light emission from the red to the blue [62]. The renewed research interest in these materials is driven largely by the desire to fabricate cheaper and more powerful electronic devices and LED's [1,62]. Ion implantation is an attractive area-selective processing tool to perform doping, etching, and electrical isolation of devices $[1,62]$. This process is widely used in the device fabrication industry, however, the process introduces lattice disorder that is often undesirable. This is the main reason for studying radiation damage in semiconductors.

Despite significant experimental and theoretical efforts, a complete understanding of damage or defect formation in either of these two materials has yet to be achieved. Previous studies on ion-implantation of GaN [63-65] have shown that the process of ion bombardment leads to layer by layer amorphization of the surface and increasing levels of disorder in the substrate bulk. This being said, the damage buildup behavior in GaN is quite complex and phenomenon such as nitrogen loss from the sample surface, ion-beam-induced porosity and anomalous surface erosion at elevated temperatures have been demonstrated [62]. GaN has been shown to be very radiation tolerant with high dynamic defect annealing that is thought to arise due to the high diffusivity of point defects and strong defect trapping at the sample 
Rev. 1.2

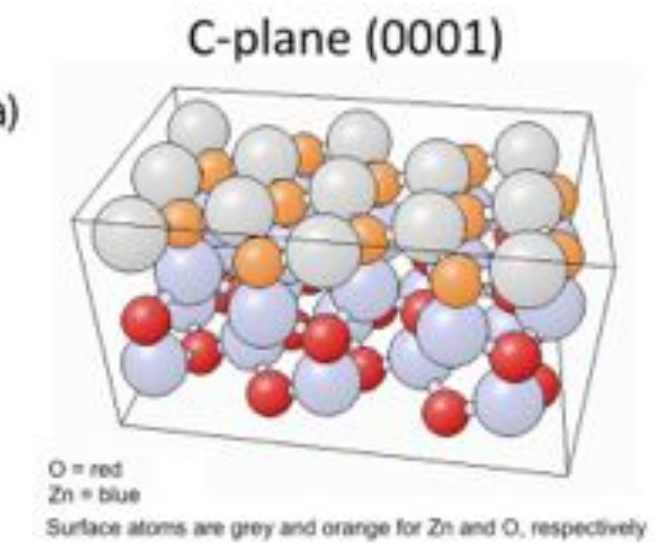

(b)
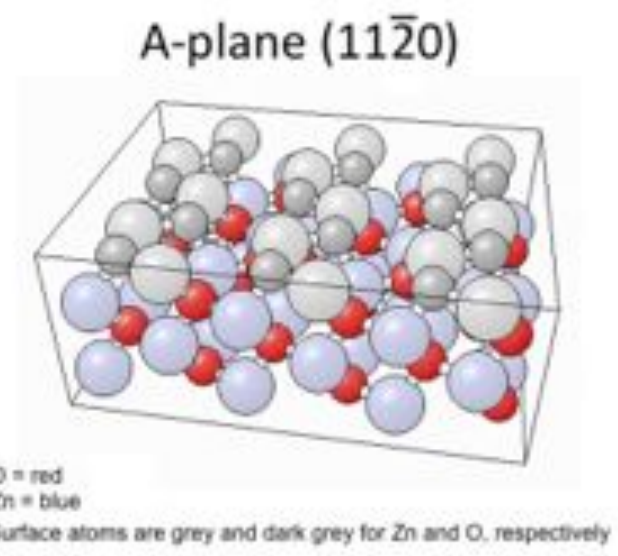

(c)

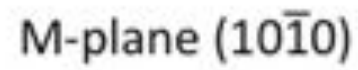

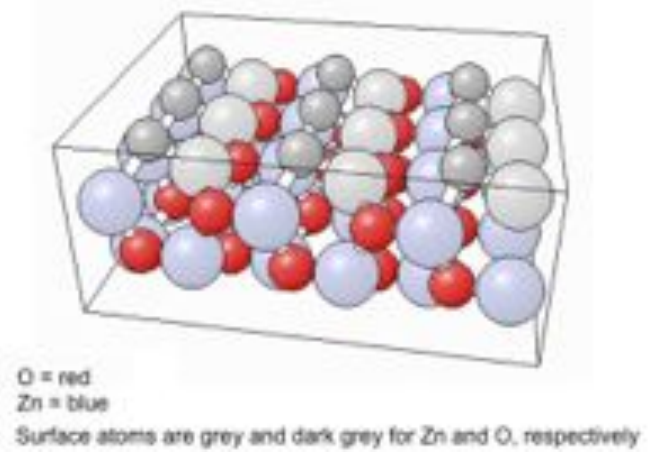

Figure 4.2: Unreconstructed surfaces of (a) polar (0001), and non-polar (b) (112̄0) and (c) (1010) ZnO. Oxygen atoms indicated by red spheres and zinc atoms by blue spheres. The color of surface atoms are indicated in the legend of each panel for both zinc and oxygen atoms. 
Rev. 1.2

surface [66].

Recently, $\mathrm{ZnO}$ has gained momentum as a major rival to GaN for transparent optoelectronic devices, including blue and UV LED's [1]. ZnO is a transparent, non-toxic semiconductor with a high exciton binding energy $(60 \mathrm{meV})$ which offers the potential for RT lasing based on exciton recombination [1]. In addition to the potential for devices, $\mathrm{ZnO}$ has been shown to be more radiation tolerant than other semiconductors of similar desirable optoelectronic properties, such as GaN. In fact, $\mathrm{ZnO}$ has substantially higher dynamic defect annealing than GaN [13]. Given the potential applications which would require ion implantation, a number of previous studies have focused on understanding buildup and annealing of implantation damage in $\mathrm{ZnO}[2-8,68]$.

It is now well established that, at $\mathrm{RT} \mathrm{ZnO}$ exhibits strong DA [2]. It has also been shown that radiation-generated elementary point defects are mobile at RT. Theoretical calculations have suggested that migration barriers for all native point defects in $\mathrm{ZnO}$ are low [67]. As a result, even high-dose irradiation with heavy ions of kiloelectronvolt energies with fluences up to $\sim 100$ DPA does not render $\mathrm{ZnO}$ amorphous at RT [2]. Interestingly, accumulation of stable post-implantation disorder in the $\mathrm{ZnO}$ bulk at depths close to the maximum nuclear energy loss is characterized by a very simple linear dependence on the number of ballistic displacements for a large dose range of $\sim 1.5-15$ DPA. In this regime, the maximum level of bulk damage exhibits a negligible dependence on sample temperature (for $15-673 \mathrm{~K}$ ), ion beam flux, and the average density of collision cascades [4-6]. The bulk damage level can, therefore, be predicted empirically based on straightforward ballistic calculations $[4,6]$.

In contrast to bulk damage, the buildup of lattice disorder near the sample surface is highly anomalous for $\mathrm{ZnO}$ and remains poorly understood. Under heavy-ion (or cluster-ion) irradiation, an unexpected intermediate peak (IP) has been observed in 
depth profiles of lattice disorder measured by high-resolution RBS/C spectrometry $[4,6,13]$. Under heavy ion irradiation, the IP clearly originates at the sample surface and moves deeper into the crystal bulk with increasing ion dose, the process that we refer to below as "IP propagation." The IP formation depends on ion mass, which has been attributed to an effect of the density of collision cascades via a currently unknown mechanism $[4,6]$. The IP in RBS/C spectra is not caused by scattering from impurities. It is also not a measurement artifact caused by planar channeling [13]. The IP is thought to originate from a narrow band of extended defects [13]. However, the atomic structure, formation mechanisms, and the driving force for its propagation are still not understood.

\subsection{The role of the surface in heavy-ion-bombarded $\mathrm{ZnO}^{\dagger}$}

In this subsection, we address the role of the sample surface in near-surface defect clustering and the formation of the IP in particular. We study structural disorder in (0001) $\mathrm{ZnO}$ single crystals bombarded at RT with $500 \mathrm{keV}$ Xe ions at different incident angles in order to control the length of the displacement generation profile without significantly altering collision cascade density. Results reveal that the $\mathrm{ZnO}$ surface influences damage buildup in the first $\sim 50 \mathrm{~nm}$, which we assign to an effective defect diffusion length. We find that, for the irradiation conditions used, the formation and propagation of the IP are independent of the length of the displacement generation profile. Results suggest that the IP layer originates from a narrow band of extended defects that, similar to the behavior of the sample surface, traps and annihilates mobile point defects in the surrounding region. Upon bombardment, the IP moves deeper into the sample until it reaches a distance from the sample $\dagger$ This work was reprinted from Scripta Materialia, Vol 67 / Issue 1, M. T. Myers, S. Charnvanichborikarn, L. Shao and S. O. Kucheyev, "Effect of the surface on ion-beam damage buildup in ZnO", Pages No. 65-68, Copyright (2012), with permission from Elsevier. 
surface comparable with an effective defect diffusion length.

Wurtzite (0001) ZnO single crystals, obtained from Cermet Inc., were implanted at RT with $500 \mathrm{keV} \mathrm{Xe} \mathrm{Xe}^{+}$ions at $7^{\circ}$ and $60^{\circ}$ off the [0001] direction to various DPA levels. This was done in order to form near-surface damage layers with a similar overall shape but with different thicknesses and without significantly altering the average density of collision cascades (which is known to influence the IP formation in $\mathrm{ZnO}$ ) [4]. During irradiation, fluxes were kept constant to maintain a constant displacement generation rate of $\sim 7 \times 10^{-3} \mathrm{DPA} / \mathrm{s}$ at a depth of the maximum nuclear energy loss $\left(R_{p d}\right)$. Ion irradiation and ion beam analysis were carried out with the 4 MV ion accelerator (National Electrostatics Corporation, model 4UH) at Lawrence Livermore National Laboratory.

The DPA values were calculated with the TRIM code (version SRIM-2008.04) [20] with threshold energies for atomic displacements of 34 and $44 \mathrm{eV}$ for $\mathrm{Zn}$ and O sublattices, respectively [69]. All DPA values are quoted as the concentration of ion-beam-generated lattice vacancies at the $R_{p d}$ normalized to the atomic concentration of $\mathrm{ZnO}\left(8.3 \times 10^{22} \text { atoms } \mathrm{cm}^{-3}\right)^{1}$. Profiles of relative concentrations of excess vacancies were defined as the difference between TRIM-predicted distributions of vacancies and interstitials normalized to the vacancy concentration at the $R_{p d}$. For a synopsis of the irradiation conditions, see Table 4.1.

Implantation-induced disorder was measured by $\mathrm{RBS} / \mathrm{C}$ with $2 \mathrm{MeV}{ }^{4} \mathrm{He}^{+}$ions incident along the [0001] direction and backscattered into a detector at $100^{\circ}$ relative to the incident beam direction, providing enhanced near-surface depth resolution. All RBS/C spectra were analyzed with one of the conventional algorithms [70] for extracting the effective number of scattering centers (referred to below as "relative

\footnotetext{
${ }^{1}$ The ion doses (in units of ions $\mathrm{cm}^{-2}$ ) used in calculations of DPAs took into account an increase in the ion beam spot area for the case of $60^{\circ}$-tilted samples.
} 
Table 4.1: A summary of the pertinent irradiation conditions used to perform irradiation of (0001) $\mathrm{ZnO}$ at $\mathrm{RT}$ with $500 \mathrm{keV} \mathrm{Xe}^{+}$ions to variable fluences at two incident ion angles.

\begin{tabular}{|l|c|c|c|c|}
\hline Angle & Flux $\left(\mathrm{cm}^{-2} \mathrm{~s}^{-1}\right)$ & Fluence $\left(\mathrm{cm}^{-2}\right)$ & DPA & DPA Rate $(\mathrm{DPA} / \mathrm{s})$ \\
\hline \hline $7^{\circ}$ & $\sim 1 \times 10^{12}$ & $1 \times 10^{15}$ & 5 & $\sim 7 \times 10^{-3}$ \\
$7^{\circ}$ & $\sim 1 \times 10^{12}$ & $2 \times 10^{15}$ & 10 & $\sim 7 \times 10^{-3}$ \\
$7^{\circ}$ & $\sim 1 \times 10^{12}$ & $3 \times 10^{15}$ & 15 & $\sim 7 \times 10^{-3}$ \\
$7^{\circ}$ & $\sim 1 \times 10^{12}$ & $6 \times 10^{15}$ & 30 & $\sim 7 \times 10^{-3}$ \\
\hline \hline $60^{\circ}$ & $\sim 5.6 \times 10^{11}$ & $5 \times 10^{14}$ & 5 & $\sim 7 \times 10^{-3}$ \\
$60^{\circ}$ & $\sim 5.6 \times 10^{11}$ & $1 \times 10^{15}$ & 10 & $\sim 7 \times 10^{-3}$ \\
$60^{\circ}$ & $\sim 5.6 \times 10^{11}$ & $1.5 \times 10^{15}$ & 15 & $\sim 7 \times 10^{-3}$ \\
$60^{\circ}$ & $\sim 5.6 \times 10^{11}$ & $3 \times 10^{15}$ & 30 & $\sim 7 \times 10^{-3}$ \\
\hline
\end{tabular}

disorder"). Deconvolution by fitting disorder depth profiles with Gaussians was used to evaluate the areal defect concentrations (in defects $/ \mathrm{cm}^{2}$ ) in IPs and surface peaks (SPs) of disorder as well as positions of IPs and bulk peaks (BPs) of disorder.

Figure 4.3(a) shows TRIM-code predicted distributions of lattice vacancies and implanted Xe atoms. The two vertical dash lines denote $R_{p d}$ 's of $68 \mathrm{~nm}$ and $25 \mathrm{~nm}$ for $7^{\circ}$ and $60^{\circ}$ cases, respectively. Also shown in Fig. 4.3(a) are profiles of ballisticallygenerated excess vacancies, with negative values indicating an excess of interstitials. It is seen from Fig. 4.3(a) that, as expected, irradiation at both angles is characterized by a unimodal Gaussian-like vacancy distribution, with the $R_{p d}$ shifted to a shallower depth with increasing ion beam angle. ${ }^{2}$ Profiles of implanted Xe atoms have maxima deeper than corresponding $R_{p d}$ 's. There is an excess of vacancies near the surface (for depths $\lesssim 90$ and $55 \mathrm{~nm}$ for $7^{\circ}$ and $60^{\circ}$ cases, respectively) and an excess of interstitials at larger depths.

Figures 4.4(a)-4.4(d) show experimentally measured profiles of relative disorder in $\mathrm{ZnO}$ bombarded at $7^{\circ}$ and $60^{\circ}$ to similar DPA at corresponding $R_{p d}$ 's, with ion

\footnotetext{
${ }^{2}$ Note that for sample tilt angles resulting in $R_{p d}$ 's comparable to or smaller than the lateral ion straggling, $R_{p d}$ 's decrease faster than the cosine dependence.
} 


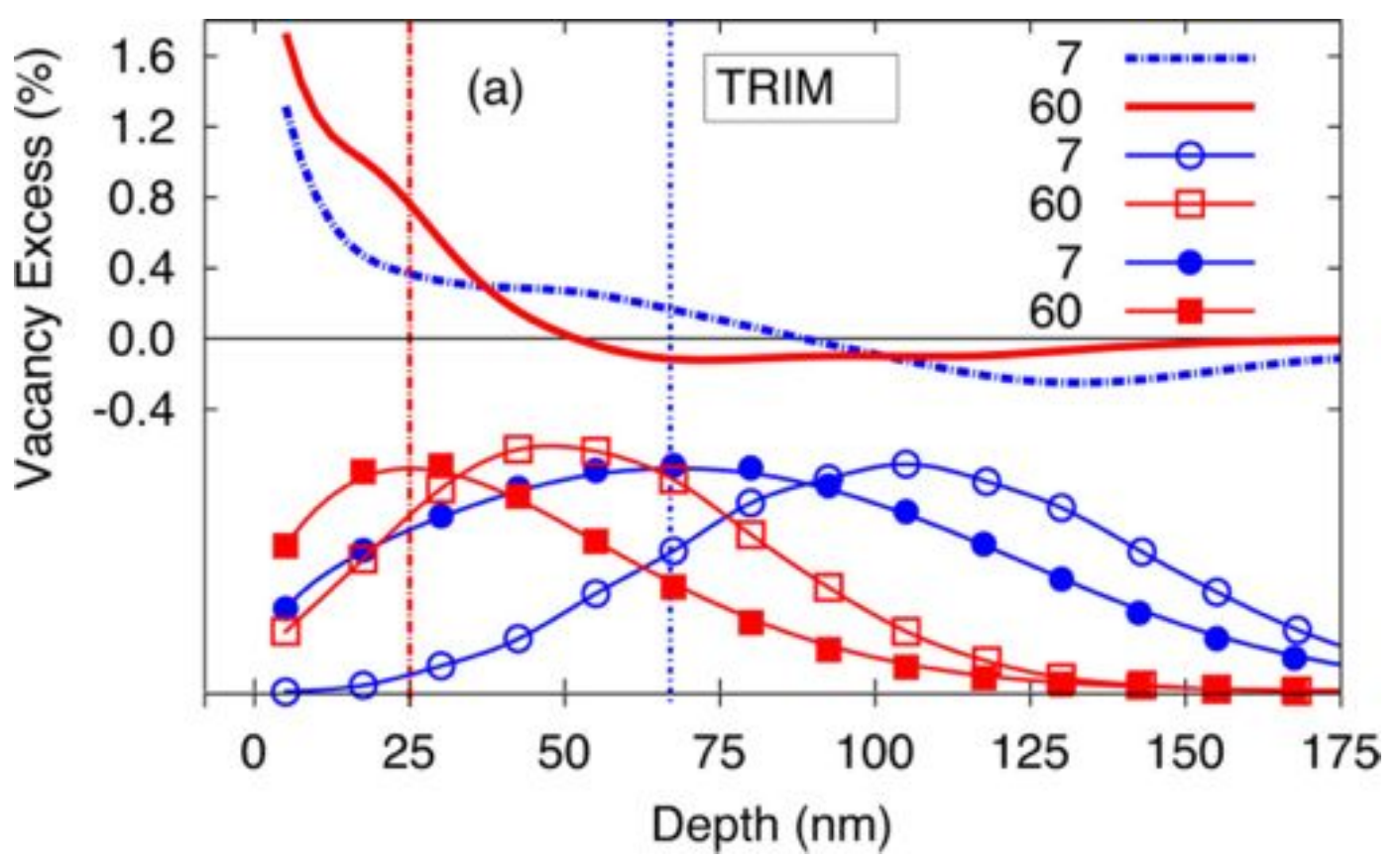

Figure 4.3: (a) Normalized TRIM-code predicted profiles of lattice vacancies (closed symbols) and implanted Xe atoms (open symbols) for $500 \mathrm{keV}$ Xe ion irradiation of $\mathrm{ZnO}$ at $7^{\circ}$ and $60^{\circ}$ off the surface normal. The vertical scale is linear and is in arbitrary units. Concentrations of excess vacancies are also shown by lines without symbols. Vertical dash lines mark the maxima of vacancy profiles, $R_{p d}$ 's, for both implantation angles. Circle and square symbols represent results for $7^{\circ}$ and $60^{\circ}$ implants, respectively. 
Rev. 1.2

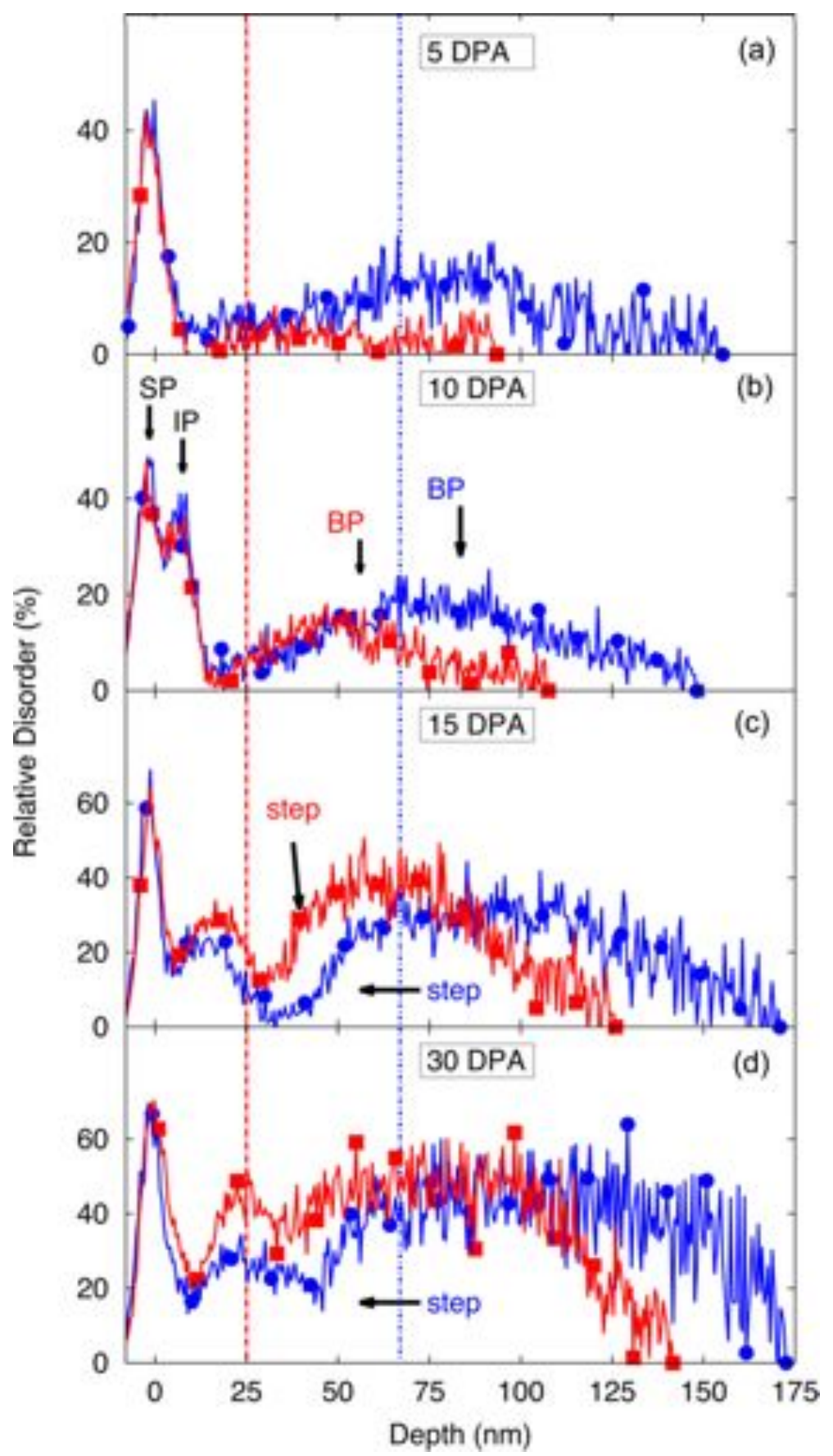

Figure 4.4: (a)-(d) Depth profiles of relative disorder in (0001) $\mathrm{ZnO}$ bombarded at RT by $500 \mathrm{keV}$ Xe ions at $7^{\circ}$ and $60^{\circ}$ off the [0001] direction to different fluences, indicated in the legends in units of DPA. For clarity, only every 20th experimental point has a symbol. In all five panels, circle and square symbols represent results for $7^{\circ}$ and $60^{\circ}$ implants, respectively. Vertical dash lines mark the maxima of vacancy profiles, $R_{p d}$ 's, for both implantation angles. 
fluences increasing from Fig. 4.4(a) to Fig. 4.4(d). Such damage profiles are rich in features and are strikingly different from the Gaussian-like distributions of ballistic displacements shown in Fig. 4.3(a). An illustrative example of this is complete lack of measurable disorder around the $R_{p d}$ in samples irradiated at $60^{\circ}$ to 5 and $10 \mathrm{DPA}$ [Figs. 4.4(a) and 4.4(b)]. Clearly resolvable, and denoted by arrows in Figs. 4.4(b)4.4(d), are the following three defect peaks and a step: the BP, SP, IP (positioned between the SP and BP), and an abrupt step located between the IP and BP [seen only in Figs. 4.4(c) and 4.4(d)].

A deviation of experimental damage profiles from TRIM-predicted curves of atomic displacements is typical for irradiation conditions with strong DA [1], which, at RT, is the case for materials with metallic or largely ionic bonding [32]. It is related to a fact that the stable lattice defects measured after irradiation are formed as a result of complex (i.e., poorly understood) processes of diffusion and interaction of the Frenkel pair components.

Figures 4.4(a)-4.4(d) show that, for both implant angles and all fluences, the BP monotonically increases with increasing dose and is located significantly deeper than corresponding $R_{p d}$ 's. Upon continuing bombardment, the BP position moves to larger depths. The evolution of the BP is better illustrated in Fig. 4.5, showing the BP magnitude [Fig. 4.5(a) (left scale)] and its depth [Fig. 4.5(b) (left scale)]. The shift of the $\mathrm{BP}$ relative to the $R_{p d}$ has been attributed to the formation of stable defects nucleating in the ion end of range, stimulated by an excess of lattice interstitials [13]. This explanation is consistent with the present results. Indeed, a comparison of Figs. 4.3(a) and 4.5(b) shows that, for both $7^{\circ}$ and $60^{\circ}$ cases, the BP positions are approaching depths of $\sim 80$ and $\sim 120 \mathrm{~nm}$, where profiles of excess interstitials have maxima.

Figure 4.5(a) (left scale) reveals that, for fluences of 5 and 10 DPA, the BP grows 


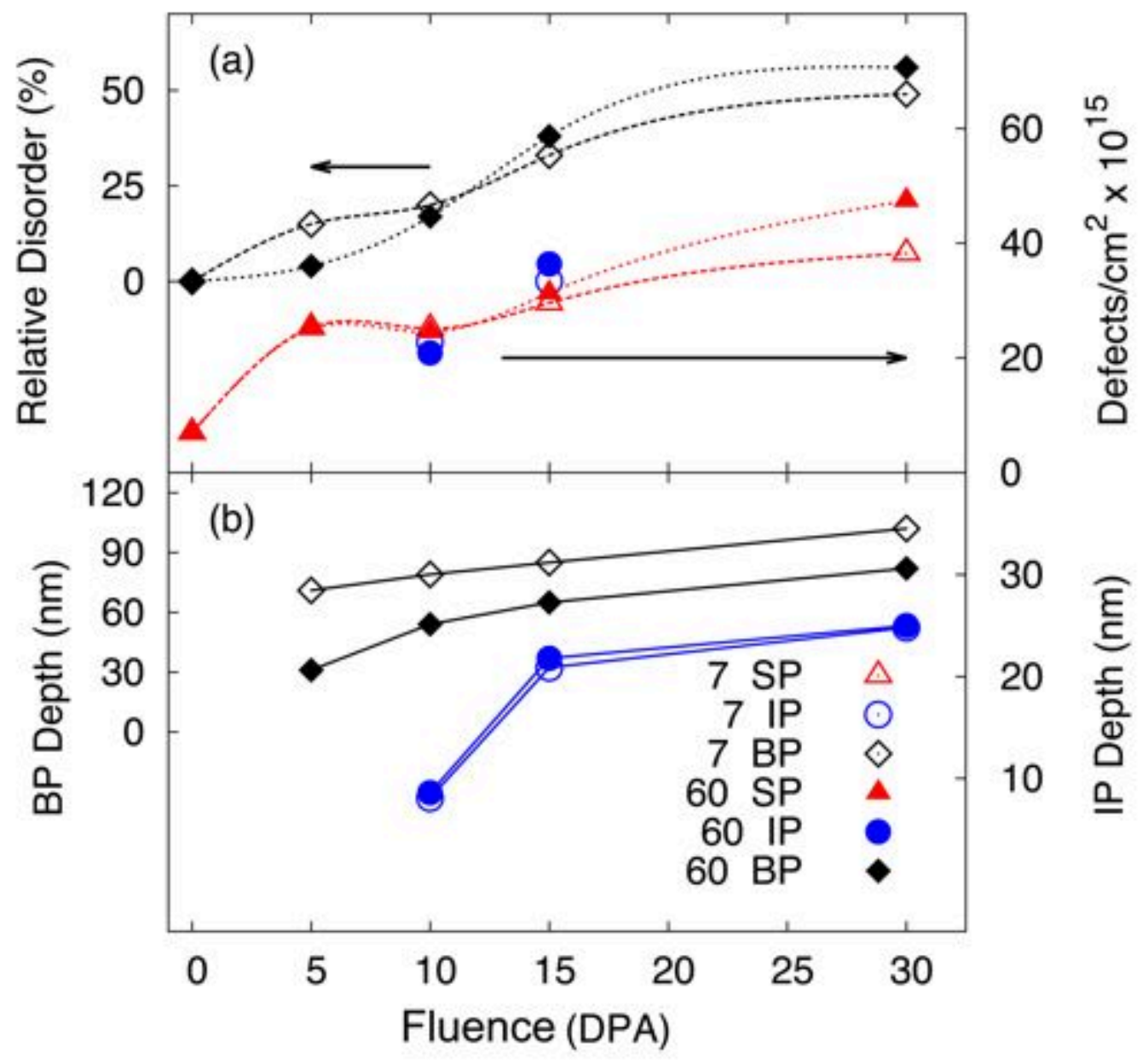

Figure 4.5: Fluence dependencies of (a) (left scale) relative disorder at the maximum of the bulk defect peak (BP), (a) (right scale) areal defect concentrations in the surface peak (SP) and the intermediate peak (IP) of disorder, and the position of (b) (left scale) the BP maximum and (b) (right scale) the IP from the sample surface. The legend in (b) applies to both panels. 
slower for $60^{\circ}$ than for $7^{\circ}$ irradiation. For this dose range, for the $60^{\circ}$ case, the $\mathrm{BP}$ is within $\sim 50 \mathrm{~nm}$ from the SP and/or the IP layer. This strongly suggests that both the $\mathrm{ZnO}$ surface and the IP layer efficiently trap and annihilate point defects within an effective diffusion length, $L_{D}$, which, given data from Fig. 4.5, could be estimated as $\sim 50 \mathrm{~nm}$.

Figure 4.5(a) (right scale) shows the dose evolution of the intensities of the SP and IP. Spectra from unimplanted samples have SPs with an area of $\sim 7 \times 10^{15}$ atoms $\mathrm{cm}^{-2}$, corresponding to about three (0001) $\mathrm{ZnO}$ monolayers. This is consistent with channeling studies of Appleton and Feldman [71], reporting surface peak thicknesses of $\sim 3$ effective monolayers. Based on results of Stensgaards et al. [72] and the Coulomb shadow cone radius and a thermal vibration amplitude of $\mathrm{ZnO}$, a SP scattering intensity of $\sim 3$ monolayers corresponds to an ideal defect-free $\mathrm{ZnO}$ surface.

It is seen from Figs. 4.4(a) and 4.5(a) that irradiation to 5 DPA leads to a large increase in the SP intensity as compared to the SP for unirradiated samples. At 10 DPA, an IP has formed, while no further increase in the SP is observed with increasing dose from 5 to 10 DPA [Fig. 4.5(a)]. It suggests that the formation of the IP significantly impacts defect diffusion which leads to no net increase in surface disorder. With increasing dose above $10 \mathrm{DPA}$, both the SP and IP grow, and the IP moves away from the surface and appears to saturate at a depth of $\gtrsim 30 \mathrm{~nm}$. This is similar to an IP saturation depth of $\sim 40 \mathrm{~nm}$ measured for $300 \mathrm{keV} \mathrm{Au}$ ion bombardment of $\mathrm{ZnO}$ [13].

Figures 4.4(a) and 4.4(b) show that the spectra for 5 and 10 DPA for both beam angles essentially overlap in the range from the surface to a depth of $\sim 50 \mathrm{~nm}$, despite a large difference in the displacement generation profiles for $7^{\circ}$ and $60^{\circ}$ cases in this near surface region [Fig. 4.3(a)]. In particular, the IP is at the same depth 
and with the same intensity for two vastly different $R_{p d}$ 's. As discussed above, the fluences used in this study have been chosen to match displacements at corresponding $R_{p d}$ 's, not at the surface. For fluences generating the same displacements at $R_{p d}$ 's, the vacancy generation at the surface is $\sim 40 \%$ larger for the $60^{\circ}$ than for the $7^{\circ}$ case. This indicates that processes of the formation and propagation of the IP are determined by displacements not at the sample surface but averaged over some nearsurface region. We have found that the curves of the IP position [shown in Fig. 4.5(b) as a function of DPA at the $R_{p d}$ ] essentially overlap when plotted as a function of the displacements averaged over a depth of $0-50 \mathrm{~nm}$, which is consistent with an $L_{D}$ of $\sim 50 \mathrm{~nm}$ discussed above.

Another interesting and novel effect revealed by Figs. 4.4(c) and 4.4(d) is that, for both implantation angles, damage profiles for 15 and 30 DPA fluences have additional abrupt steps [denoted by arrows in Figs. 4.4(c) and 4.4(d)]. Such steps are located at $\sim 30$ and $20 \mathrm{~nm}$ from the IP for $7^{\circ}$ and $60^{\circ}$ cases, respectively. A dramatic decrease in the disorder level in the region between the IP and the step is clearly seen in Fig. 4.4(c) for both curves. Moreover, a comparison of Figs. 4.4(b) and 4.4(c) reveals that, for the $7^{\circ}$ implant, the damage level between the IP and the step (at a depth of $\sim 40 \mathrm{~nm}$ ) decreases with increasing dose from 10 to 15 DPA. It indicates the existence of some (currently unknown) defect interaction processes that lead to an annihilation of otherwise stable disorder within $\sim 20-30 \mathrm{~nm}$ from the IP layer. It is also seen from Fig. 4.4(c) that, for $7^{\circ}$ as compared to the $60^{\circ}$ case, in addition to a wider (by $\sim 10 \mathrm{~nm}$ ) plateau with reduced disorder, the disorder level at the plateau is lower. These observations could be attributed to a closer proximity of the $\mathrm{BP}$ and a larger local vacancy generation for the $60^{\circ}$ than for the $7^{\circ}$ case. The fact that the width of the plateau $(\sim 20-30 \mathrm{~nm})$ is smaller than the $L_{D}(\sim 50 \mathrm{~nm})$ estimated above for the lower dose regime is also consistent with a notion that the $L_{D}$ is not 
a constant. The $L_{D}$ is defined by defect trapping at some defect sinks. Its value depends not only on sample temperature but also on the concentration and type of stable defects present in the crystal lattice. Shorter $L_{D}$ 's are expected for regimes with larger damage levels, which is consistent with our observations.

The present study has clearly shown that the IP is a near-surface phenomenon. The sample surface is essential for its formation and existence. The importance of the surface is further supported by the fact that both the IP position and intensity are essentially the same for irradiation conditions with about a factor of two different sputtering rates [73-75]. The sputtering yield was calculated with the TRIM code [20] with values of surface binding energies adjusted to $3.2 \mathrm{eV}$ for both $\mathrm{Zn}$ and $\mathrm{O}$ in order to match the experimental sputtering yield data for ZnO reported in Ref. [73] for 30 keV Ga ion irradiation and in Ref. [74] for $1-4 \mathrm{keV}$ Ar ion irradiation (results from both Refs. [73] and [74] agreed with each other). According to such calculations, the sputtering rates are $\sim 0.2$ and $\sim 0.5 \mathrm{~nm} / \mathrm{DPA}$ for $7^{\circ}$ and $60^{\circ}$, respectively. Note that such TRIM-code predictions are consistent with Sigmund's theory [75], predicting that, with increasing tilt angle, the sputtering yield increases faster than the inverse cosine dependence. Indeed, the inverse cosine dependence would result in essentially the same sputtering rate (in units of nm $\mathrm{DPA}^{-1}$ ) for different tilt angles since both the sputtering yield (in units of atoms per ion) and the ion dose (in units of ions $\mathrm{cm}^{-2}$ ) needed to reach a given DPA level at the $R_{p d}$ follow cosine dependencies on the tilt angle. We also note that relative sputtering rates at $7^{\circ}$ and $60^{\circ}$ are not affected by nonlinear sputtering effects due to a constant average cascade density in such experiments.

We could also now eliminate the role of the following two processes speculated earlier as possible contributors to the formation and evolution of the IP [13]. First, given a significant difference in the ballistic separation of vacancies and interstitials 
in the near-surface region for $7^{\circ}$ and $60^{\circ}$ cases [Fig. 4.3(a)] and an almost identical formation and evolution of the IP (Fig. 4.5), this effect is not the major driving force for the IP formation. Second, preferential sputtering could also be excluded since, for the $60^{\circ}$ case, the total ballistic sputtering is about two times larger than for $7^{\circ}$ implants.

Finally, we note that, although the near-surface damage buildup revealed in $\mathrm{ZnO}$ is anomalous, it is not unique. Indeed, previous studies of a number of compound non-metallic materials, including $\mathrm{SiC}$ [76], $\mathrm{UO}_{2}$ [77], CdTe [78], and $\mathrm{UN}$ [79], have revealed IPs [80-83]. Convincing explanations for this effect are still lacking, and further studies aimed at understanding such intriguing and complex phenomena are currently needed. In particular, future high-resolution depth profiling of lattice damage could provide some answers. Note that the IP, the focus of the present study, should not be confused with anomalous peaks previously observed in channeling spectra of $\mathrm{SiC}$ and $\mathrm{Si}$ only in cases when the channel is not parallel to the surface normal [80-83]. Such anomalous peaks move toward the surface with increasing ion dose. This effect has been attributed to the lattice strain caused by ion-beam-induced material swelling [80].

The results of this work could be summarized as follows:

(i) The $\mathrm{ZnO}$ surface strongly influences damage buildup in the first $\sim 50 \mathrm{~nm}$ from the surface, which we have assigned to an effective defect diffusion length.

(ii) The intermediate peak located between the surface and bulk peaks of disorder is a surface effect, whose formation and propagation are independent of the length of the radiation damage profile (for the irradiation conditions used here).

(iii) Similar to the behavior of the $\mathrm{ZnO}$ surface, the IP layer efficiently traps and annihilates mobile point defects. As the dose is increased and the IP moves deeper 
into the bulk, the IP could promote defect recombination in a narrow layer. This results in the formation of an abrupt step between the IP and the BP in the depth distribution of stable lattice disorder.

(iv) Upon continuing irradiation, the IP moves into the crystal bulk until it reaches a distance from the sample surface comparable with an effective defect diffusion length.

4.3 Defect microstructure in heavy-ion-bombarded (0001) $\mathrm{ZnO} \ddagger$

Ion-beam-produced disorder in $\mathrm{ZnO}$ has been a subject of a number of previous studies [1-11]. Previous experiments have shown that, for a wide range of irradiation conditions, the level of stable post-implantation disorder in the $\mathrm{ZnO}$ crystal bulk, measured by RBS/C spectrometry, depends linearly on the concentration of ballistically generated lattice displacements [4-6]. However, the buildup of damage in the first $\sim 50 \mathrm{~nm}$ from the sample surface is complex and cannot be readily predicted. In particular, an anomalous intermediate peak (IP) has been observed in depth profiles of lattice disorder in (0001) $\mathrm{ZnO}$ irradiated with heavy or cluster ions and measured by high-resolution RBS/C $[4,6,11,13]$. The IP is located at a depth between the expected surface and bulk peaks of disorder. The IP moves deeper into the bulk with increasing ion dose. This movement is also accompanied by the formation of an abrupt step in between the IP and the bulk peak [11]. The sample surface plays a vital role in the IP formation, and the IP itself has been attributed to a narrow band of extended defects that efficiently trap and annihilate mobile point defects [11]. However, despite a number of previous systematic studies $[4,6,11,13]$, the mechanisms by which the IP forms have remained elusive.

In this subsection, we correlate previously unexplained RBS/C observations [11]

$\ddagger$ This work was reprinted from Acta Materialia, Vol 60 / Issue 17, M. T. Myers, S. Charnvanichborikarn, C.C. Wei, Z.P. Luo, A. Aitkaliyeva, L. Shao and S. O. Kucheyev, "Defect microstructure in heavy-ion-bombarded (0001) ZnO", Pages No. 6086-6090, Copyright (2012), with permission from Elsevier. 
Table 4.2: Irradiation conditions and relevant parameters used to perform irradiation of (0001) $\mathrm{ZnO}$ at $\mathrm{RT}$ with $500 \mathrm{keV} \mathrm{Xe}{ }^{+}$ions to variable fluences. * Denotes that this data has been reported previously.

\begin{tabular}{|l|c|c|c|c|}
\hline Angle & Flux $\left(\mathrm{cm}^{-2} \mathrm{~s}^{-1}\right)$ & Fluence $\left(\mathrm{cm}^{-2}\right)$ & DPA & DPA Rate $(\mathrm{DPA} / \mathrm{s})$ \\
\hline \hline $60^{\circ \star}$ & $\sim 5.6 \times 10^{11}$ & $5 \times 10^{14}$ & 5 & $\sim 7 \times 10^{-3}$ \\
$60^{\circ \star}$ & $\sim 5.6 \times 10^{11}$ & $1.5 \times 10^{15}$ & 15 & $\sim 7 \times 10^{-3}$ \\
\hline
\end{tabular}

with the microstructure studied by cross-sectional transmission electron microscopy (XTEM) and scanning TEM (STEM) using a high-angle annular dark-field (HAADF) detector. Results reveal that the damage evolution proceeds via the formation of a band of cavities centered on $\sim 7 \mathrm{~nm}$ from the sample surface. With further irradiation, a layered structure is formed, with alternating near-stoichiometric and Zn-rich layers. The anomalous intermediate peak and step in ion channeling spectra are attributed to a Zn-rich defect band and an interface between stoichiometric and Znrich layers, respectively. To explain these observations, we propose a damage buildup scenario involving vacancy clustering, loss of $\mathrm{O}$ from the surface, and peculiarities of point defect transport through a Zn-rich defect band toward the surface.

Two wurtzite (0001) ZnO single crystals, obtained from Cermet Inc., were bombarded at RT with $500 \mathrm{keV} \mathrm{Xe}^{+}$ions at $60^{\circ}$ off the [0001] direction to 5 and 15 DPA with a constant displacement generation rate of $\sim 7 \times 10^{-3} \mathrm{DPA} / \mathrm{s}$. Actual ion fluences were 0.5 and $1.5 \times 10^{15} \mathrm{~cm}^{-2}$, and the beam flux was held constant at $5.6 \times 10^{11}$ $\mathrm{cm}^{-2} \mathrm{~s}^{-1}$. Ion irradiation and ion beam analysis were carried out with the $4 \mathrm{MV}$ ion accelerator (National Electrostatics Corporation, model 4UH) at Lawrence Livermore National Laboratory. The DPA values were calculated, as previously [4, 6, 11], with the TRIM code (version SRIM-2008.04) [20] with threshold energies for atomic displacements of 34 and $44 \mathrm{eV}$ for $\mathrm{Zn}$ and $\mathrm{O}$ sublattices, respectively. All DPA values are quoted as the concentration of ion-beam-generated lattice vacancies at a depth of the maximum nuclear energy loss $(25 \mathrm{~nm})$ normalized to the atomic concentration of 
$\mathrm{ZnO}\left(8.3 \times 10^{22}\right.$ atoms $\left.\mathrm{cm}^{-3}\right)$. For a synopsis of the irradiation conditions, see Table 4.2. Depth profiles of implantation-produced disorder for these two samples, measured by RBS/C with $2 \mathrm{MeV}^{4} \mathrm{He}^{+}$ions, were reported previously [11]. Specimens for XTEM were prepared by applying a conventional sandwich technique followed by dimple polishing and $3 \mathrm{keV}$ Ar ion milling in a Gatan 600/691 PIPS. Imaging (in TEM and STEM modes) and energy dispersive spectroscopy (EDS) analysis were performed in an FEI TECNAI G² F20 ST operated at $200 \mathrm{kV}$.

Figure 4.6(a) shows a XTEM image overlaid with the depth profile of disorder extracted from RBS/C spectra for the sample bombarded to 5 DPA. The overlay shows that the depth profile of disorder is bimodal, consisting of a surface peak (labeled SP) and a broad bulk peak (labeled BP). The XTEM image of Fig. 4.6(a) reveals a near-surface band of cavities. The fact that these are indeed cavities is supported by XTEM images shown in Figs. 4.6(b) and 4.6(c), comparing in-focus and under-focused imaging conditions, respectively, of different regions from the same sample (bombarded to 5 DPA). The top white arrow in Fig. 4.6(b) marks a band of interconnected $\sim 3$-nm-wide cavities centered on $\sim 7 \mathrm{~nm}$ from the sample surface. The bottom white arrow in Fig. 4.6(b) points to a slightly larger diameter cavity that extends to the sample surface. Also visible in Fig. 4.6(b) are several other dark diffraction-contrast features possibly originating from some irradiationproduced lattice defects. The under-focused XTEM image in Fig. 4.6(c) clearly shows that the near-surface cavities are faceted, with cavity walls occupying particular

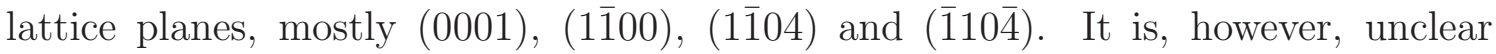
whether the cavities are empty or filled with gas (presumably $\mathrm{O}_{2}$ ).

Figure 4.7(a) shows a XTEM image overlaid with a RBS/C-derived depth profile of disorder for the 15 DPA sample. Clearly resolved in the damage profile are the following features: the SP, IP, BP, and a step between the IP and BP. No near-surface 
Rev. 1.2
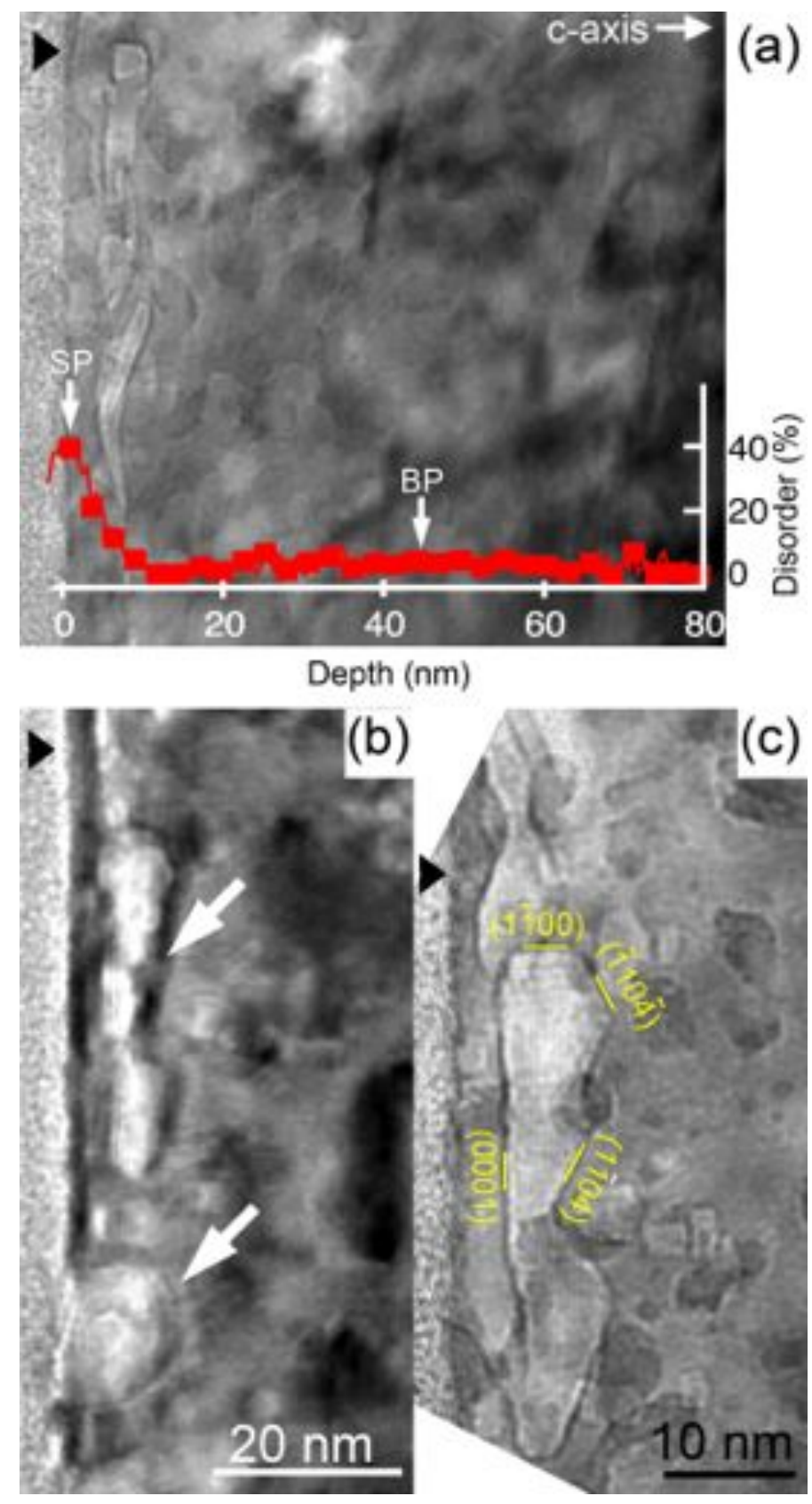

Figure 4.6: (a) XTEM image (slightly under-focused, around $11 \overline{2} 0$ zone axis) of $\mathrm{ZnO}$ bombarded to 5 DPA. Overlay in (a) is the depth profile of relative disorder measured by RBS/C, where only every 5th experimental point is depicted for clarity. The depth scale of the RBS/C spectrum also applies to the image in (a). (b)-(c) Higher resolution XTEM images of the same sample. Image (b) is in focus, around the $11 \overline{2} 0$ zone axis. Image (c) is under-focused, slightly off the $11 \overline{2} 0$ zone axis. The sample surface is shown by black triangles. The $c$-axis shown by a horizontal white arrow in (a) applies to all three images. Bombardment was done at RT with $500 \mathrm{keV}$ Xe ions at $60^{\circ}$ off the [0001] direction. 
Rev. 1.2
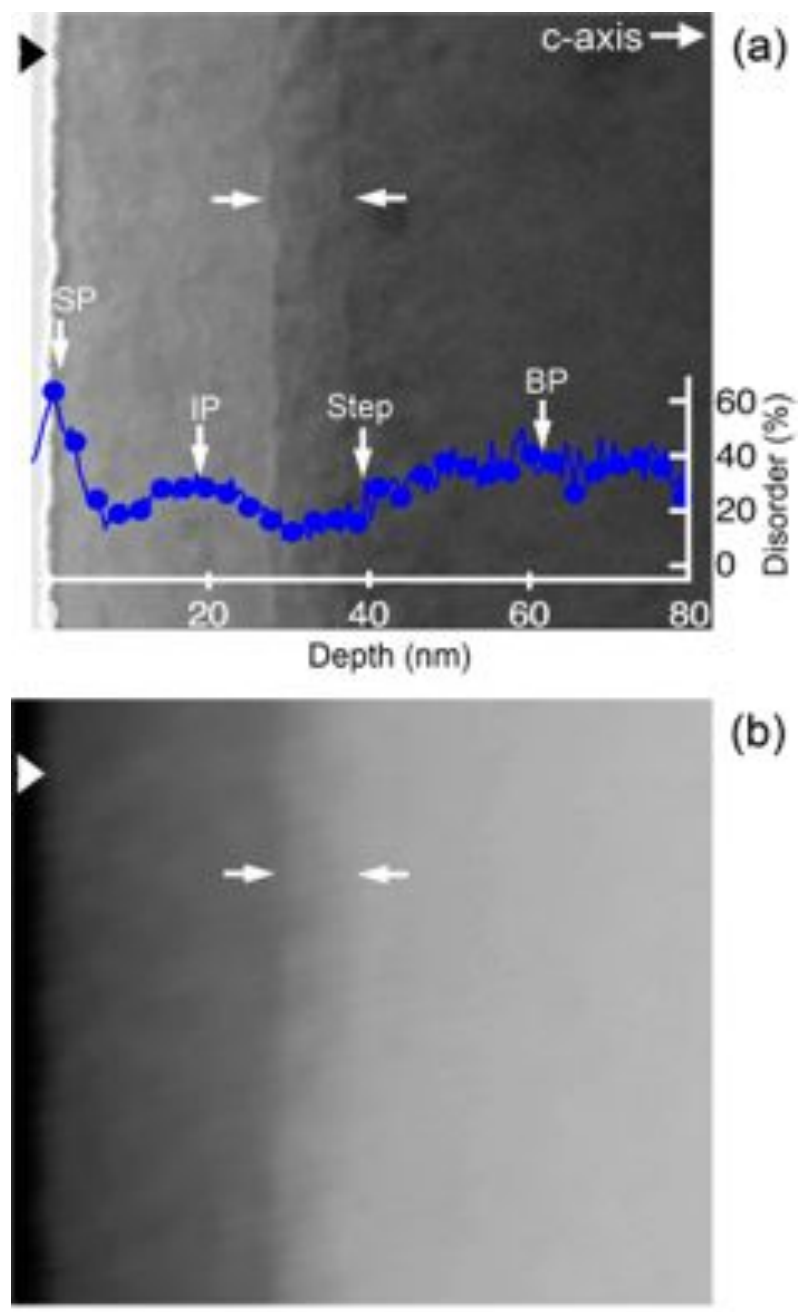

(b)

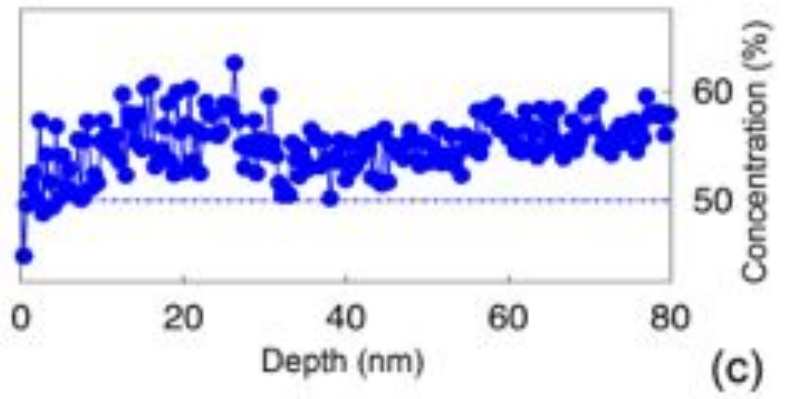

Figure 4.7: (a) XTEM image (in-focus, around $11 \overline{2} 0$ zone axis) of $\mathrm{ZnO}$ bombarded to 15 DPA. Overlay in (a) is the depth profile of relative disorder measured by RBS/C, where only every 5th experimental point is depicted for clarity. (b) HAADF STEM image of the 15 DPA sample. (c) Concentration of Zn as a function of depth obtained from a STEM/EDS line scan of (b). Sample surface is shown by triangles in (a) and (b). The $c$-axis is marked with a horizontal white arrow, and the depth scale of the RBS/C spectrum applies to both images in (a) and (b). Bombardment was done at RT with $500 \mathrm{keV}$ Xe ions at $60^{\circ}$ off the [0001] direction. 
cavities are observed in the XTEM image of Fig. 4.7(a), in contrast to the case of the lower dose (5 DPA) sample. This indicates that the cavities have either collapsed or exfoliated from the surface on increasing dose from 5 to 15 DPA. Instead, Fig. 4.7(a) reveals a structure consisting of three well-defined layers, with the sample surface marked by a black triangle and two interfaces by horizontal white arrows. The first layer with a brighter contrast starts at the sample surface and is $\sim 27 \mathrm{~nm}$ thick. It corresponds to the combined SP and IP regions of the RBS/C spectrum. The second layer is $\sim 10 \mathrm{~nm}$ thick. It corresponds to a plateau between the IP and the step in the overlay. The third layer starts at the step at $\sim 37 \mathrm{~nm}$ from the surface and extends over the BP region. ${ }^{3}$

Figure 4.7(b) shows a HAADF STEM image of the 15 DPA sample, again revealing a layered structure although in a reversed contrast compared to the XTEM image of Fig. 4.7(a). In STEM/HAADF imaging mode, the contrast is proportional to the sample mass $(Z$ contrast). Hence, the $\sim 27 \mathrm{~nm}$ thick near surface layer in Fig. 4.7(b) is of a lower density than that of the other two layers. This layer also exhibits a speckle contrast consisting of adjacent regions of lighter and darker contrast, indicating density variations.

Figure 4.7(c) is the depth profile of the Zn concentration obtained from a line scan performed using STEM/EDS for the 15 DPA sample. It shows that the $\sim 5$-nm-thick near-surface layer is essentially stoichiometric. An increase in the Zn concentration is observed in the region of $\sim 5-20 \mathrm{~nm}$ from the surface, corresponding to the depths where the IP is present in the RBS/C spectrum in Fig 4.7(a). In the layer located at $\sim 30-40 \mathrm{~nm}$ from the surface, corresponding to the plateau in the RBS/C

\footnotetext{
${ }^{3}$ The formation of a layered structure having two distinct bands is consistent with previously unexplained results [9] reported for $\mathrm{ZnO}$ bombarded with $300 \mathrm{keV}$ As ions at RT to a very high dose of $1.4 \times 10^{17} \mathrm{~cm}^{-2}$, with a brighter contrast band extending from the surface to $\sim 30 \mathrm{~nm}$ and a band of a darker contrast beyond that depth.
} 
spectrum in Fig. 4.7(a), the Zn concentration is nearly stoichiometric. In the third layer, for depths $\gtrsim 40 \mathrm{~nm}$, the sample is again Zn-rich. Such an observation of a stoichiometric near surface layer followed by a Zn-rich region is in agreement with previous photoemission studies [13] of (0001) ZnO crystals irradiated at RT with 300 $\mathrm{keV} \mathrm{Au}$ ions to a dose of $2 \times 10^{16} \mathrm{~cm}^{-2}$.

Figure 4.8(a) shows a higher magnification XTEM image of the 15 DPA sample, revealing a peculiar "droplet-like" contrast. As shown in Fig. 4.8(b), regions closest to the sample surface exhibit a brighter contrast and have a large number of $\lesssim 1 \mathrm{~nm}$ bright contrast dots [marked by arrows], which could be small cavities. With increasing depth, the density of such bright dots decreases, leading to a darker contrast. This observation is in agreement with the HAADF XTEM image of Fig. 4.7(b), which shows that the near surface layer has a reduced density. Figure 4.8(c) is a XTEM image of the region defined by the white square frame in Fig. 4.8(a). It shows that the droplet-like contrast arises from strain fields due to defect-induced lattice distortion and what appear to be small cavities $\lesssim 1 \mathrm{~nm}$ in diameter [marked by arrows in Fig. 4.8(c)] that bound the edge of the "droplet-like" feature. Lattice distortions are better illustrated by Fig. 4.8(d), showing an inverse Fourier transform of the image of Fig. 4.8(c) with only $\pm(0002)$ reflections selected [84-86]. Interestingly, the processed XTEM image of Fig. 4.7(d) is similar to images reported by Matzke and Wang [84] for $\mathrm{UO}_{2}$ polycrystals irradiated with $500 \mathrm{keV} \mathrm{Xe}$ ions at $\sim 170^{\circ} \mathrm{C}$ to a dose of $5 \times 10^{15} \mathrm{~cm}^{-2}$. Urania is a somewhat similar material to $\mathrm{ZnO}$ : both are non-amorphizable oxides. Matzke and Wang [84] have assigned the XTEM contrast to the formation of crystallites with $\sim 1-2^{\circ}$ misorientation and attributed it to polygonization. Despite a similar looking contrast in XTEM images from ZnO and $\mathrm{UO}_{2}$, we have found no evidence of any polycrystallization or polygonization in the RT Xe-irradiated ZnO samples studied here $[85,86]$. The RBS/C derived profile 
Rev. 1.2

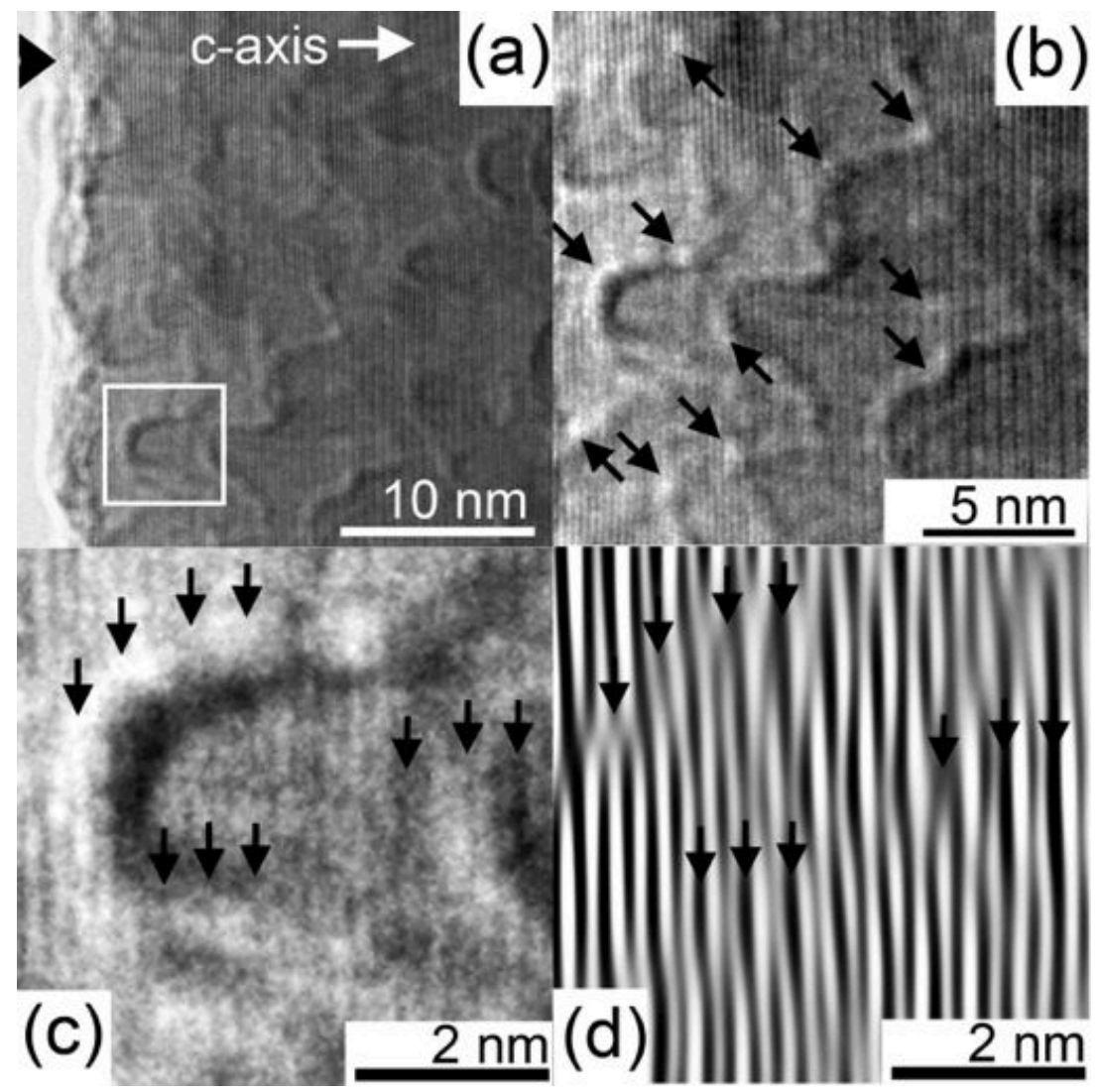

Figure 4.8: (a) XTEM image (slightly off the $11 \overline{2} 0$ zone axis) of $\mathrm{ZnO}$ bombarded to 15 DPA. (b) Higher magnification Fourier filtered XTEM image of (a) with the left edge starting at a depth of $\sim 2 \mathrm{~nm}$ from the sample surface. (c) Fourier filtered XTEM image of the region defined by the white square in (a). (d) Inverse Fourier transform of (c) with only $\pm(0002)$ reflections selected. The $c$-axis shown by a horizontal white arrow in (a) applies to all images. The sample surface is shown by a black triangle in (a). Bombardment was done at RT with $500 \mathrm{keV}$ Xe ions at $60^{\circ}$ off the [0001] direction. 
shown in Fig. 4.6(b) shows that the 15 DPA sample is still highly $c$-axis oriented.

Based on the above results and the previously established fact that the IP formation requires high-density collision cascades $[4,6,13]$, we propose the following damage buildup scenario. Dense collision cascades create energy spikes, including thermal and displacement spikes [87]. As discussed previously [11], the near-surface layer is enriched in vacancies, with excess interstitials at larger depths. A high local concentration of vacancies after thermalization of dense collision cascades could lead to the formation of vacancy clusters $[88,89]$ that, upon continuing irradiation, evolve into cavities. Thermal spikes are also likely to cause material decomposition, accompanied by loss of oxygen from the sample surface [13] and from the walls of cavities. As cavities collapse or exfoliate from the surface, left behind is a Zn rich near-surface region. A comparison of Figs. 4.6(b) and 4.8(a) reveals that the surface roughness of the $15 \mathrm{DPA}$ sample is substantially greater than that of the 5 DPA sample, supporting that cavities exfoliate from the surface.

Continued irradiation leads to the movement of the IP deeper into the crystal bulk and the formation of a layered structure revealed by Figs. 4.7(a) and 4.7(b). This behavior could be understood as follows. Since the $\mathrm{ZnO}$ surface is an efficient defect sink [11], there is a near-surface gradient of instantaneous concentrations of mobile defects. This drives defect diffusion toward the surface. Theoretical calculations have suggested that migration barriers for all native point defects in $\mathrm{ZnO}$ are low [67] The movement of the IP (i.e., the Zn-rich defect band) could be caused by peculiarities of its interaction with a flux of point defects diffusing through it toward the sample surface. An effective Zn transport accompanying IP movement could be related to differences in mobilities of different point defects diffusing through the Znrich band. For example, trapping of $\mathrm{O}$ vacancies at the Zn-rich band would result in its effective propagation into the bulk, while Zn vacancies could also result in 
the same IP movement when they exit the Zn-rich band (on the way to the sample surface) as $\mathrm{O}$ vacancies. This scenario is consistent with previous observations that the IP saturates at $\sim 30-40 \mathrm{~nm}$ from the sample surface $[11,13]$. Indeed, the IP moves to a depth comparable with the effective defect diffusion length, where the defect concentration gradient diminishes. Although the above scenario is plausible, more work is currently needed to fully understand atomic-level mechanisms of defect accumulation in ion-irradiated $\mathrm{ZnO}$.

In summary, we have shown that irradiation of $\mathrm{ZnO}$ with heavy ions at $\mathrm{RT}$ causes the formation of near-surface cavities and Zn-rich layers, giving rise to an anomalous peak and a step in ion channeling spectra. The formation of a band of cavities has been attributed to vacancy clustering and/or material decomposition in dense collision cascades. The movement of the Zn-rich band deeper into the crystal bulk with continued irradiation could be understood based on expected differences of the mobilities of elemental point defects through such a band toward the sample surface. These results for $\mathrm{ZnO}$ could provide insight for understanding somewhat similar anomalous near-surface damage previously observed in a number of other compound non-metallic materials, including $\mathrm{SiC}$ [76], $\mathrm{UO}_{2}$ [77], CdTe [78], and UN [79].

\subsection{Tailoring radiation damage in (0001) $\mathrm{ZnO}$ by surface modification}

The present subsection attempts to address a fundamental question associated with heavy-ion irradiation of (0001) ZnO crystals. Given such a strong effect of the free surface on radiation damage in (0001) $\mathrm{ZnO}$, can the damage buildup be controlled by manipulating the surface properties? We demonstrate that the nearsurface damage of (0001) $\mathrm{ZnO}$ can indeed be suppressed by placing a thin layer of $\mathrm{AlO}(\mathrm{OH})$ on the sample surface. By using ion channeling and transmission electron microscopy, we find that placing an $\sim 7$-nm-thick $\mathrm{AlO}(\mathrm{OH})$ layer on the (0001) $\mathrm{ZnO}$ 
surface results in (i) suppression of cavity formation, (ii) a reduced intermediate defect peak intensity, and (iii) a decreased level of disorder extending down to $\sim 100$ $\mathrm{nm}$ from the sample surface for room-temperature bombardment with $500 \mathrm{keV}$ Xe ions. Our results demonstrate the potential to control radiation damage in $\mathrm{ZnO}$ by surface manipulation.

Heavy-ion irradiation of (0001) $\mathrm{ZnO}$ crystals results in unusual damage buildup, including an additional (intermediate) peak in damage-depth profiles measured by ion channeling, the formation of near-surface nanocavities, and stoichiometric imbalance. All these effects are thought to be associated with the influence of the sample surface on DA processes. The (0001) ZnO surface appears to act as a sink for mobile point defects, playing a vital role in the evolution of near-surface stable disorder $[1,2$, $4,6,7,9,11-13,90]$. Damage buildup in ion channeling spectra proceeds via the formation of an intermediate peak (IP) located between the expected surface and bulk defect peaks $[4,6,11-13]$. The IP has been associated with a Zn-rich region [12]. With increasing ion dose, the IP moves deeper into the bulk. For irradiation with $500 \mathrm{keV}$ Xe ions, the IP position saturates at depths of $\sim 40-50 \mathrm{~nm}$ independent of incident ion angles (i.e., the projected ion range) [11, 12], suggesting that the IP formation and movement with increasing dose are influenced by the presence of the sample surface. In addition to nonstoichiometric (Zn-rich) regions, nanoscale cavities have been observed near the surface in (0001) $\mathrm{ZnO}$ crystals bombarded at room-temperature with $500 \mathrm{keV} \mathrm{Xe}$ ions [12].

Wurtzite (0001) ZnO single crystals, obtained from Cermet Inc., were used. Selected crystals were coated with an $\sim 7 \mathrm{~nm}$ thick conformal layer of $\mathrm{AlO}(\mathrm{OH})$ by alternating exposures to trimethyl-aluminum (TMA) and water precursors in a warm wall atomic layer deposition reactor (Kurt J. Lesker), as described elsewhere [91]. During the deposition, reactor walls and the sample stage were kept at $125^{\circ} \mathrm{C}$, while 
the sample was repeatedly exposed to the following sequence: TMA pulse, Ar purge, $\mathrm{H}_{2} \mathrm{O}$ pulse, and Ar purge. The pressure during TMA and $\mathrm{H}_{2} \mathrm{O}$ pulses was 0.8 Torr and 1.2 Torr, respectively.

Implantation was performed at RT with $500 \mathrm{keV}^{129} \mathrm{Xe}^{+}$ions at $60^{\circ}$ off the [0001] direction to 5 and $15 \mathrm{DPA}$, which correspond to Xe ion fluences of $5 \times 10^{14}$ and $1.5 \times 10^{15} \mathrm{~cm}^{-2}$, respectively, for both bare (i.e., uncoated) samples and those coated with $\mathrm{AlO}(\mathrm{OH})$ by $\mathrm{ALD}$. During irradiation, flux values $\left(\sim 5 \times 10^{11} \mathrm{~cm}^{-2} \mathrm{~s}^{-1}\right)$ were kept constant to maintain a constant displacement generation rate of $\sim 7 \times 10^{-3}$ $\mathrm{DPA} / \mathrm{s}$ at a depth of the maximum nuclear energy loss $\left(R_{p d} \cong 25 \mathrm{~nm}\right)$. Ion irradiation and ion beam analysis were carried out with the $4 \mathrm{MV}$ ion accelerator (National Electrostatics Corporation, model 4UH) at Lawrence Livermore National Laboratory. The DPA values are quoted as the concentration of ion-beam-generated lattice vacancies at the $R_{p d}$ normalized to the atomic concentration of $\mathrm{ZnO}\left(8.3 \times 10^{22}\right.$ atoms $\mathrm{cm}^{-3}$ ) and were calculated with the TRIM code (version SRIM-2008.04) [20] with threshold energies for atomic displacements of 34 and $44 \mathrm{eV}$ for $\mathrm{Zn}$ and $\mathrm{O}$ sublattices, respectively [69]. For a synopsis of the irradiation conditions, see Table 4.3.

Implantation-induced disorder (in the $\mathrm{Zn}$ sublattice) was measured by RBS/C spectrometry with $2 \mathrm{MeV}^{4} \mathrm{He}^{+}$ions incident along the [0001] direction and backscattered into a detector at $100^{\circ}$ relative to the incident beam direction, providing enhanced near-surface depth resolution. All RBS/C spectra were analyzed with one of the conventional algorithms [70] for extracting the effective number of scattering centers (referred to below as "relative disorder"). Specimens for cross-sectional transmission electron microscopy (XTEM) were prepared by applying the conventional sandwich technique followed by dimple polishing and $3 \mathrm{keV}$ low angle $\mathrm{Ar}^{+}$-ion milling (Gatan 691 PIPS). XTEM specimens were then investigated in an FEI TECNAI G ${ }^{2}$ F20 ST operated at $200 \mathrm{kV}$. 


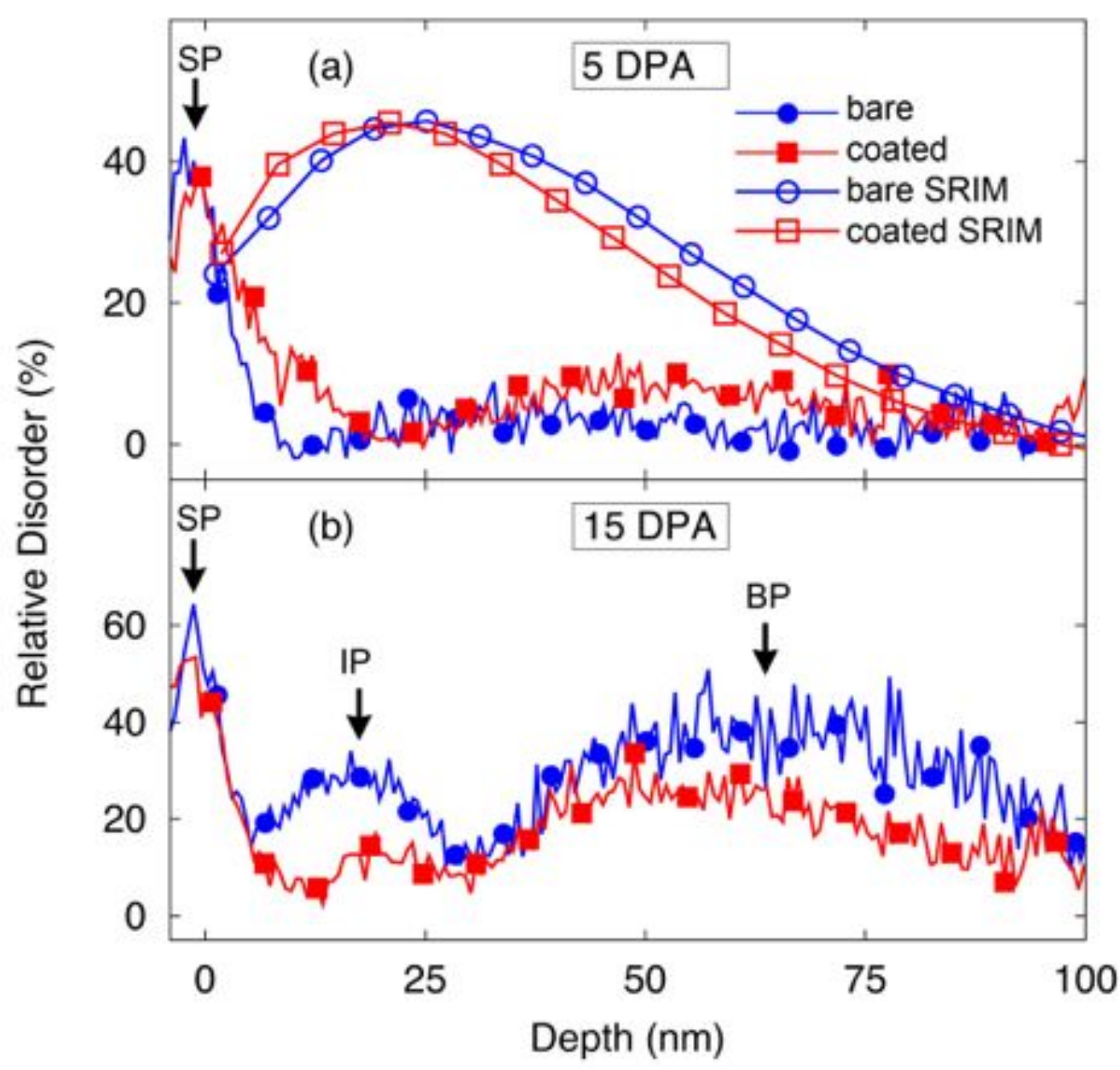

Figure 4.9: Depth profiles of relative disorder in (0001) ZnO bombarded at RT by $500 \mathrm{keV}$ Xe ions at $60^{\circ}$ off the surface normal to (a) 5 and (b) 15 DPA. For clarity, only every 10th experimental point has a symbol. In both (a) and (b), circle and square symbols represent results for bare $\mathrm{ZnO}$ (denoted as "bare") and ZnO coated with an $~ 7$-nm-thick $\mathrm{AlO}(\mathrm{OH})$ layer (denoted as "coated"), respectively. Centers of surface, intermediate, and bulk defect peaks are indicated by arrows and labeled SP, IP, and BP, respectively. Normalized vacancy generation profiles predicted by TRIM-code calculations are also shown in (a) by open symbols, with circles for bare $\mathrm{ZnO}$ and squares for coated $\mathrm{ZnO}$. 


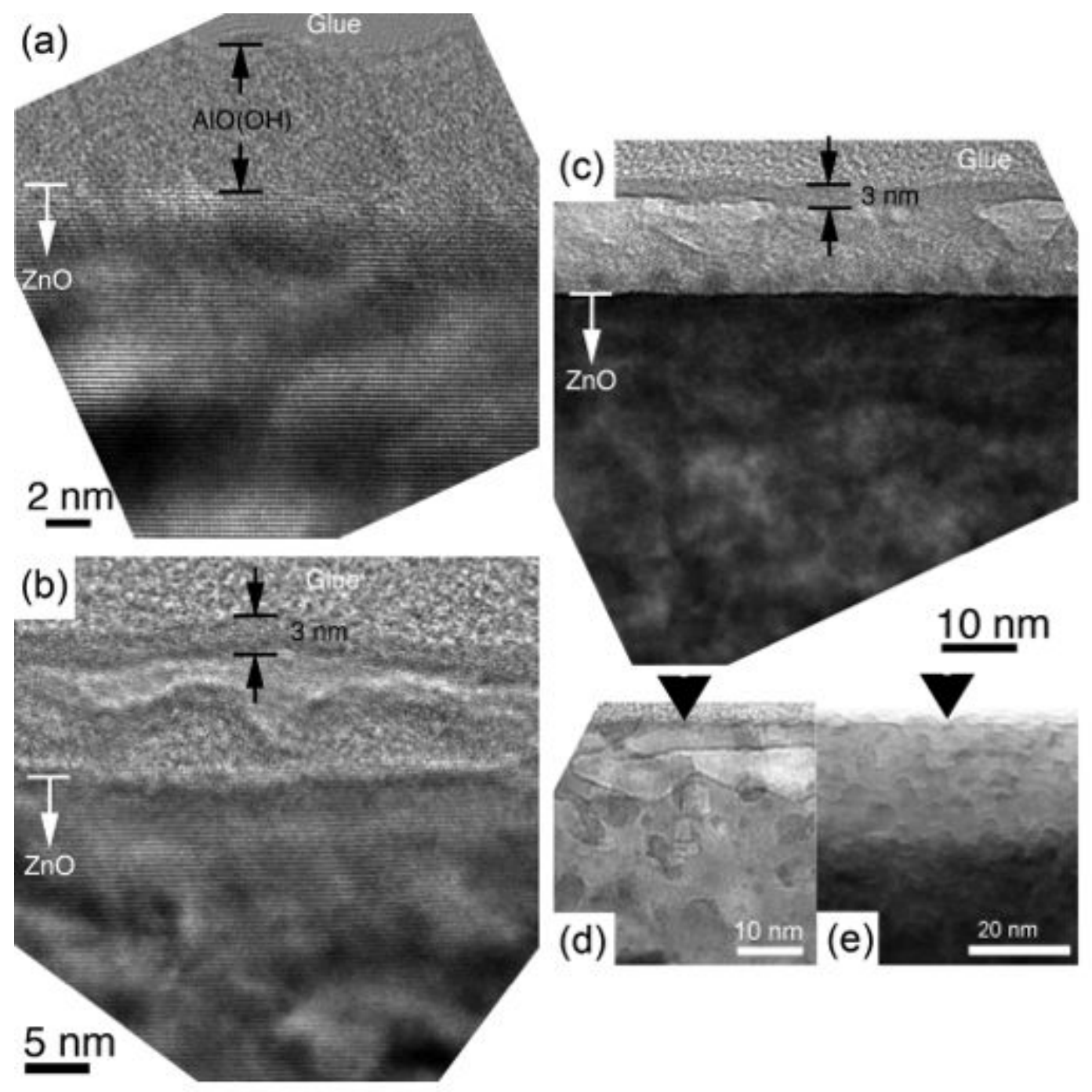

Figure 4.10: XTEM images of (0001) ZnO with [(a)-(c)] and without $[(\mathrm{d}),(\mathrm{e})]$ an $\sim 7$-nm-thick $\mathrm{AlO}(\mathrm{OH})$ layer deposited on top of the $\mathrm{ZnO}$ surface before ion bombardment. The sample before irradiation is shown in (a). Samples irradiated to 5 DPA are shown in (b) and (d), while (c) and (e) are images of samples irradiated to 15 DPA. All images were taken around the $11 \overline{2} 0$ zone axis, and irradiation was performed at RT by $500 \mathrm{keV}$ Xe ions at $60^{\circ}$ off the surface normal. The (0001) ZnO surface is marked by a horizontal white line with an arrow in $(\mathrm{a}-\mathrm{c})$ and by black triangles in $(\mathrm{d}-\mathrm{e})$. 
Table 4.3: For both coated and uncoated (0001) ZnO, a summary of the irradiation conditions used to perform RT bombardment with $500 \mathrm{keV} \mathrm{Xe}{ }^{+}$ions to 5 and 15 DPA. * Denotes that this data has been reported previously.

\begin{tabular}{|l|c|c|c|c|c|}
\hline Angle & $\mathrm{AlO}(\mathrm{OH})$ & Flux $\left(\mathrm{cm}^{-2} \mathrm{~s}^{-1}\right)$ & Fluence $\left(\mathrm{cm}^{-2}\right)$ & DPA & DPA Rate $(\mathrm{DPA} / \mathrm{s})$ \\
\hline \hline $60^{\circ}$ & yes & $\sim 5.6 \times 10^{11}$ & $5 \times 10^{14}$ & 5 & $\sim 7 \times 10^{-3}$ \\
$60^{\circ}$ & yes & $\sim 5.6 \times 10^{11}$ & $1.5 \times 10^{15}$ & 15 & $\sim 7 \times 10^{-3}$ \\
\hline \hline $60^{\circ \star}$ & no & $\sim 5.6 \times 10^{11}$ & $5 \times 10^{14}$ & 5 & $\sim 7 \times 10^{-3}$ \\
$60^{\circ \star}$ & no & $\sim 5.6 \times 10^{11}$ & $1.5 \times 10^{15}$ & 15 & $\sim 7 \times 10^{-3}$ \\
\hline
\end{tabular}

Figures 4.9(a) and 4.9(b) show profiles of relative disorder (in the Zn sublattice) in both bare and coated samples bombarded with $500 \mathrm{keV}$ Xe ions to 5 and 15 DPA, respectively. ${ }^{4}$ Clearly resolved in both Figs. 4.9(a) and 4.9(b) are surface peaks (SPs) corresponding to the $\mathrm{ZnO}$ free surface and the $\mathrm{ZnO} / \mathrm{AlO}(\mathrm{OH})$ interface for bare and coated samples, respectively. The SPs have similar scattering properties for bare and coated samples for each dose set. This indicates that the presence of an $\mathrm{AlO}(\mathrm{OH})$ coating layer has a minor influence on the damage accumulation at the $\mathrm{ZnO}$ surface/interface. For 5 DPA samples [Fig. 4.9(a)], only SPs are clearly resolved, with bulk defect peaks (BP) being almost indistinguishable from the experimental noise. However, for the 15 DPA case [Fig. 4.9(b)], in addition to SPs, BPs are clearly resolved, and the coated sample exhibits reduced BP disorder levels at depths up to $\sim 100 \mathrm{~nm}$.

For both bare and coated samples irradiated to 15 DPA, Fig. 4.9(b) also reveals an IP positioned between the SP and BP. The IP intensity for the coated sample is, however, significantly lower compared to that for the bare counterpart. A recent report [12] has suggested that both the $\mathrm{ZnO}$ surface and the IP region act as efficient sinks for radiation-generated mobile point defects, promoting annihilation of vacan-

${ }^{4}$ Details of RBS/C and XTEM characterization of bare (0001) ZnO samples irradiated under the same conditions ( $500 \mathrm{keV}$ Xe at RT to 5 and $15 \mathrm{DPA}$ at the $R_{p d}$ ) have been reported previously [12]. 
cies and interstitials. The fact that coated samples exhibit a reduced level of disorder in both the IP and $\mathrm{BP}$ further supports the assumption that the $\mathrm{ZnO}$ surface (or the $\mathrm{ZnO} / \mathrm{AlO}(\mathrm{OH})$ interface) indeed behaves as a defect sink.

Figure 4.10(a) shows a XTEM image of unirradiated (0001) ZnO coated with $\mathrm{AlO}(\mathrm{OH})$. It reveals an $\mathrm{AlO}(\mathrm{OH})$ thickness of $\sim 7 \mathrm{~nm}$, which is consistent with that measured with RBS by monitoring the energy shift of the Zn edge. By performing high-resolution XTEM imaging of the sample shown in Fig. 4.10(a) we have found that the ALD-deposited $\mathrm{AlO}(\mathrm{OH})$ layer is amorphous, which is expected based on previous ALD studies [91].

Figures 4.10(b) and 4.10(d) show XTEM images of coated and bare samples, respectively, irradiated to $5 \mathrm{DPA}$. These correspond to RBS/C-derived depth profiles of disorder depicted in Fig. 4.9(a) and discussed above. A comparison of Figs. 4.10(b) and 4.10(d) clearly demonstrates that, although their disorder depth profiles [Fig. 4.9(a)] appear similar, the defect microstructure of these two samples is dramatically different. The bare sample [Fig. 4.10(d)] contains a band of interconnected nanocavities centered on $\sim 7 \mathrm{~nm}$ from the sample surface. In contrast, no evidence of cavities or extended defects is found in the coated $\mathrm{ZnO}$ crystal [Fig. 4.10(b)]. The formation of cavities in Xe-ion-irradiated bare (0001) ZnO crystals has previously been attributed to vacancy clustering $[12,88,89]$ and/or material decomposition after the thermalization of dense collision cascades [12]. The suppression of nanocavity formation in alumina-coated crystals revealed in the present work suggests the dominating role of vacancy clustering processes. Indeed, the material decomposition within dense collision cascades is expected to be independent of the surface condition.

Figures 4.10(c) and 4.10(e) show XTEM images of coated and bare samples, respectively, irradiated to 15 DPA, corresponding to disorder depth profiles of Fig. 4.9(b). 
Again, similar to the 5 DPA case discussed above, a comparison of Figs. 4.10(c) and 4.10(e) reveals that $\mathrm{AlO}(\mathrm{OH})$ coating has a dramatic effect on the radiation-induced defect microstructure in the 15 DPA sample set. In particular, both the layered structure [12] and the droplet-like contrast [Fig. 4.10(e)] that are present in the bare $\mathrm{ZnO}$ sample irradiated to $15 \mathrm{DPA}$ are absent from the coated sample. Instead, Fig. 4.10(c) reveals a faint band of darker contrast centered on $\sim 20 \mathrm{~nm}$ from the $\mathrm{ZnO} / \mathrm{AlO}(\mathrm{OH})$ interface in the coated sample irradiated to $15 \mathrm{DPA}$.

A comparison of Figs. 4.10(a), 4.10(b), and 4.10(c) also reveals pronounced and complex irradiation-induced changes in the structure of the surface alumina layer. Some implantation-damage-related contrast and evidence of cavity formation and associated swelling of alumina are visible. For example, in the sample irradiated to 15 DPA [Fig. 4.10(c)], cavities of $\sim 5-10 \mathrm{~nm}$ in width are present. Shown above the $\mathrm{ZnO} /$ alumina interface (toward the surface) of Fig. 4.10(c) are small crystallites (in darker contrast). These could be attributed to ion-beam-induced mixing, leading to the formation of an aluminum zinc oxide alloy. Also seen is an increase in the thickness of the alumina layer after irradiation to 15 DPA [ $11 \mathrm{~nm}$, Fig. 4.10(c)] compared to that before irradiation $[\sim 7 \mathrm{~nm}$, Fig. $4.10(\mathrm{a})]$. This could be attributed to radiation-induced swelling of alumina, which is also consistent with previous reports $[92,93]$. Interestingly, our RBS measurements have revealed negligible irradiationinduced changes in the areal density of aluminum (monitored by the position of the Zn edge and the intensity of the $\mathrm{Al}$ peak). This suggests negligible sputtering with increasing ion dose from 5 to 15 DPA of the alumina layer, which is in contrast to our estimates of the ballistic sputtering yield. The sputtering yield of $\sim 0.5 \mathrm{~nm} / \mathrm{DPA}$ has been estimated for bare $\mathrm{ZnO}$ irradiated (at $60^{\circ}$ off normal) with $500 \mathrm{keV} \mathrm{Xe}$ ions [11]. The sputtering yield of alumina is roughly half that of $\mathrm{ZnO}$ [74], suggesting that $\sim 1$ and $\sim 4 \mathrm{~nm}$ of alumina are expected to be sputtered for 5 and 15 
DPA, respectively. Figures 4.10(b) and 4.10(c), however, reveal the presence of an $\sim 3$-nm-thick layer near the surface. The formation of such a surface layer could arise from an irradiation-assisted deposition of hydrocarbons. Clearly, the structure and complex evolution of the alumina layer under irradiation revealed here deserves further studies.

The strong effect of an $\mathrm{AlO}(\mathrm{OH})$ coating layer on sub-surface damage evolution in (0001) ZnO crystals demonstrated here is intriguing. The suppression of near-surface damage (including both BP and IP) as well as the formation of nanocavities could be related to several complex phenomena including (i) modification of the surface properties related to peculiarities of the surface/interface interaction with mobile point defects, (ii) a suppression of O loss from $\mathrm{ZnO}$, (iii) mechanical stresses related to differences in the thermal expansion of $\mathrm{ZnO}$ and $\mathrm{AlO}(\mathrm{OH})$, and (iv) the influence of the polar (0001) $\mathrm{ZnO}$ surface on defect diffusion. Our current data is insufficient to differentiate between these scenarios, and additional systematic studies are needed. Nevertheless, our results have straightforward implications for designing methods to mitigate radiation damage in solids via manipulation of surface and interface properties.

In summary, we have demonstrated that radiation damage in $\mathrm{ZnO}$ can be controlled by surface manipulation. Coating (0001) $\mathrm{ZnO}$ with a thin conformal $\mathrm{AlO}(\mathrm{OH})$ layer before heavy-ion irradiation can suppress the formation of nanocavities and significantly decrease the level of disorder extending up to $\sim 100 \mathrm{~nm}$ from the sample surface.

4.5 Dynamic annealing differences in polar and non-polar $\mathrm{ZnO}$

Most studies on radiation damage in $\mathrm{ZnO}$ focus on the polar surface termination (0001), however, understanding radiation damage buildup in non-polar surfaces is 
highly desirable. This is due to the fact that polarization associated with the $\mathrm{ZnO}$ (0001) surface results in the quantum confined Stark effect (QCSE) which oftentimes reduces the intrinsic quantum efficiency of devices. Heavy ion irradiation of polar (0001) $\mathrm{ZnO}$ has been shown to lead to the formation of an anomalous intermediate peak (IP) in ion channeling spectra. The IP is Zn-rich defect band whose origin is closely correlated to the free surface. Such highly unusual behavior in (0001) $\mathrm{ZnO}$ necessitates the study of ion beam induced damage buildup in other non-polar $\mathrm{ZnO}$ facets which are being extensively investigated for device fabrication. Here,

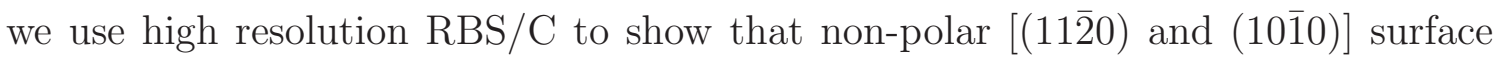
terminations of $\mathrm{ZnO}$ bombarded with $500 \mathrm{keV}$ Xe ions exhibit enhanced radiation tolerance as compared to polar (0001) $\mathrm{ZnO}$. In addition, the formation of an IP in non-polar orientations is completely suppressed and damage accumulation (in the bulk and surface) is substantially reduced compared to polar surface terminations irradiated under the same conditions.

Recently, research on $\mathrm{ZnO}$ has gained substantial momentum as a possible competitor to GaN for transparent optoelectronic devices, including blue and UV light emitting diodes (LEDs) [1]. ZnO is a transparent, non-toxic semiconductor with a high exciton binding energy $(60 \mathrm{meV})$ which offers the potential for RT lasing based on exciton recombination [1]. Most of the current literature has focused on polar $\mathrm{ZnO}$ grown on c-plane (0001) sapphire substrates [94-96]. The lack of an inversion center in the wurtzite structure results in the formation of a polar surface along the $C$-axis (0001) [61]. Cleaving $\mathrm{ZnO}$ along this direction results in a strong piezoelectric and spontaneous polarization leading to a built-in electric field. This phenomenon is generally referred to as the quantum-confined Stark effect (QCSE) [97-99]. Such polarity compensation can result in complex surface behavior, such as partially filling of the electronic states and adsorption of foreign species. Polarization effects 
also have profound implications on devices due to the QCSE, which causes a spatial separation of the electron and hole wave functions, restricts carrier recombination efficiency, and reduces oscillator strength [100].

Due to detrimental effect of the polar surface, substantial efforts have been undertaken to demonstrate non-polar films. [101-104] For the case of GaN-based LEDs, the internal quantum efficiency has been shown to increase to $50 \%$ by growing the LED on non-polar substrates [105]. Oftentimes, ion implantation is used as an areaselective processing tool to perform doping, etching, and electrical isolation $[1,62]$. Therefore, in addition to understanding film growth morphologies, being able to predict structural disorder as a result of ion implantation is essential. Despite significant experimental efforts, a complete understanding of damage accumulation in either GaN or ZnO has yet to be achieved. Previous studies on ion-implantation of polar GaN have shown that the process of ion bombardment leads to layer by layer amorphization of the (0001) surface and increasing levels of disorder in the substrate bulk [62-65].

In spite of the obvious advantages of non-polar films, very little literature exists (if any at all) pertaining to ion beam damage buildup in non-polar GaN or $\mathrm{ZnO}$. One recent report has shown substantial variations in the DA efficiency of polar versus non-polar GaN, an intriguing result [106]. Such dynamic annealing differences are observed experimentally as a reduced level of disorder when non-polar orientations are bombarded relative to the polar orientation (with the same irradiation conditions). Other studies have revealed a similar type of behavior in the compound semiconductors $\mathrm{MgO}$ and $\mathrm{Al}_{2} \mathrm{O}_{3}$ [107-111].

Zinc oxide has been shown to have substantially higher dynamic defect annealing and is non-amorphizable while it remains stoichiometric [13]. Numerous studies have focused on the buildup and annealing of implantation damage in $\mathrm{ZnO}$, however, the 
focus has been on polar terminated surfaces $[1-8,11,12]$. It has been observed that ion beam induced damage in the $\mathrm{ZnO}$ bulk (at depths close to the maximum nuclear energy loss) can be predicted empirically based on straightforward ballistic calculations.

However, the buildup of lattice disorder near the sample surface is highly anomalous for (0001) $\mathrm{ZnO}$ irradiated with heavy-ions. An unexpected intermediate peak (IP) has been observed in depth profiles of lattice disorder measured by high-resolution RBS/C spectrometry $[4,6,11-13]$. The IP clearly originates at the sample surface (via an unknown mechanism) and moves deeper into the crystal bulk with increasing ion fluence. For irradiation with $500 \mathrm{keV}$ Xe ions, the IP position saturates at depths of $\sim 40-50 \mathrm{~nm}$ independent of the projected ion range and is strongly correlated to the $(0001)$ free surface. $[11,12]$ The IP has been shown to be associated with a Zn-rich region which is preceded by nanoscale cavities and results in substantial microstructural changes [11, 12].

Given the large role of the free surface in heavy ion bombarded $\mathrm{ZnO}$, it is reasonable to question whether all free surfaces will behave similarly, or whether the polar surface is an exception? Zinc oxide crystals (both polar and non-polar orientations) are simultaneously bombarded with $500 \mathrm{keV}$ Xe ions and it is observed that non-

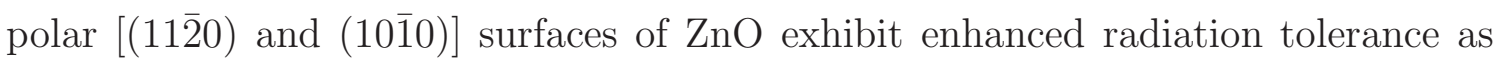
compared to polar (0001) ZnO. The non-polar surfaces do not reveal the formation of an IP which supports the claim that the polar free surface (0001) plays a vital role in IP formation, defect diffusion, and DA under irradiation.

Wurtzite $\mathrm{ZnO}$ single crystals (oriented and polished) of three different facets

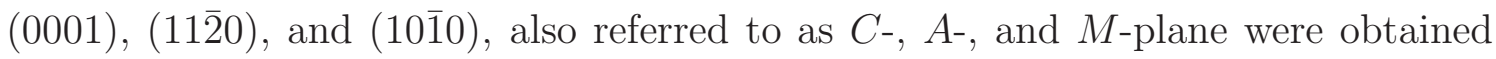
from MTI Corp. Ion irradiation and ion beam analysis were carried out with the 4 MV ion accelerator (National Electrostatics Corporation, model 4UH) at Lawrence 
Livermore National Laboratory. Bombardment was performed at RT with $500 \mathrm{keV}$ $\mathrm{Xe}^{+}$ions at $7^{\circ}$ off the [0001], [11200], and [1010] directions for $C_{-}, A_{-}$-, and $M$-plane facets, respectively. The ion fluence ranged from 5 to $30 \mathrm{DPA}$ for each of the three orientations. Beam flux values were held constant at $1.0 \times 10^{12} \mathrm{~cm}^{-2} \mathrm{~s}^{-1}\left(\sim 7 \times 10^{-3}\right.$ $\mathrm{DPA} / \mathrm{s}$ ) and the actual ion fluence ranged from 1.0 to $6.0 \times 10^{15} \mathrm{~cm}^{-2}$.

The DPA values were calculated, as previously [11, 12], with the TRIM code (version SRIM-2008.04) [20] with threshold energies for atomic displacements of 34 and $44 \mathrm{eV}$ for $\mathrm{Zn}$ and $\mathrm{O}$ sublattices, respectively. All DPA values are quoted as the concentration of ion-beam-generated lattice vacancies at a depth of the maximum nuclear energy loss $(68 \mathrm{~nm})$ normalized to the atomic concentration of $\mathrm{ZnO}\left(8.3 \times 10^{22}\right.$ atoms $\left.\mathrm{cm}^{-3}\right)$.

Implantation-induced disorder was measured by RBS/C with $2 \mathrm{MeV}^{4} \mathrm{He}^{+}$ions incident along the [0001], [11 20$]$, and [10̄0] directions for $C$-, $A$-, and $M$-plane samples, respectively. The probing beam was backscattered into a detector at $99^{\circ}$ relative to the incident beam direction, providing enhanced near-surface depth resolution. All RBS/C spectra were analyzed with a conventional algorithm [70] for extracting the effective number of scattering centers (referred to below as "relative disorder").

Figure 4.11(a-c) shows the crystallographic channels presented to the probing $\mathrm{He}$

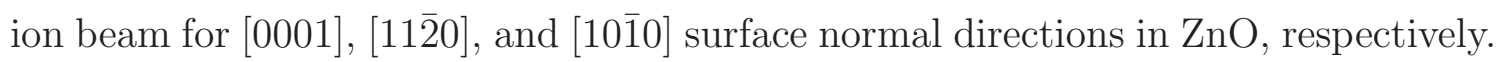
The $\mathrm{ZnO}$ wurtzite structure consists of two interconnecting sub-lattices of $\mathrm{Zn}^{2+}$ and $\mathrm{O}^{2-}$ ions. Each $\mathrm{Zn}$ ion is surrounded by a tetrahedra of $\mathrm{O}$ ions, and vice-versa. The tetrahedral coordination produces a polar symmetry along the $C$-axis. The most common surface terminations of $\mathrm{ZnO}$ consist of the polar surface [Fig. 4.11(a)], and two non-polar terminations [Figs. 4.11(b) and 4.11(c)]. Fig. 4.11(d) shows the

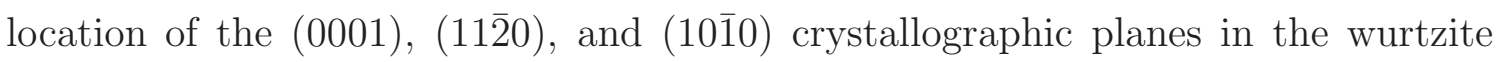
structure, shown as green, blue, and red, respectively. 
Rev. 1.2
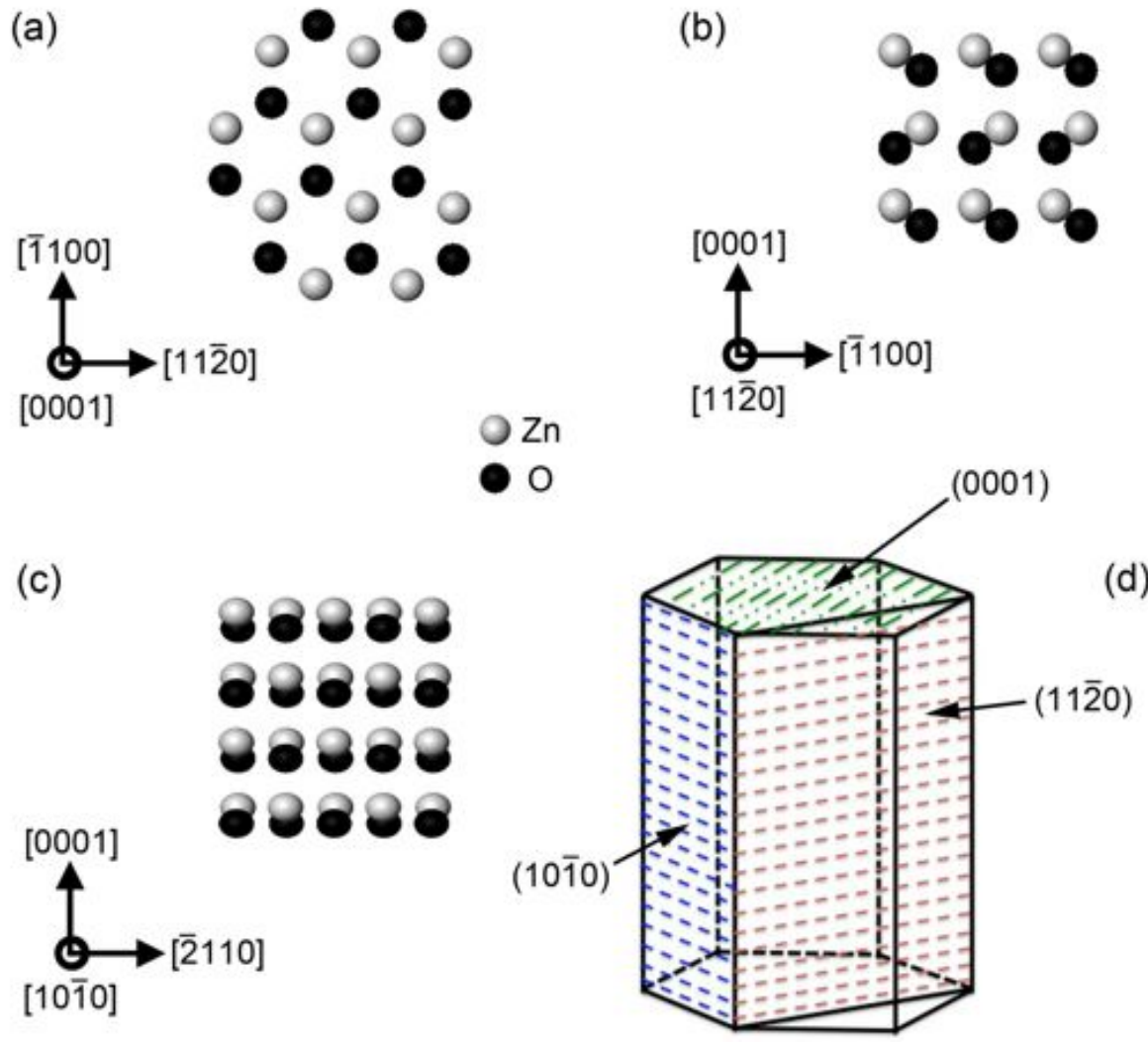

Figure 4.11: Atomic models of the crystallographic orientations and channels presented to the ion beam for the (a) [0001], (b) [1120], and (c) [1010] surface normal directions in $\mathrm{ZnO}$. White spheres represent $\mathrm{Zn}$ atoms while black spheres represent

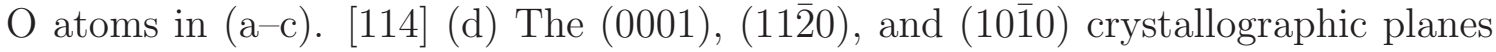
in the wurtzite structure, shown as green, blue, and red, respectively. 
Rev. 1.2

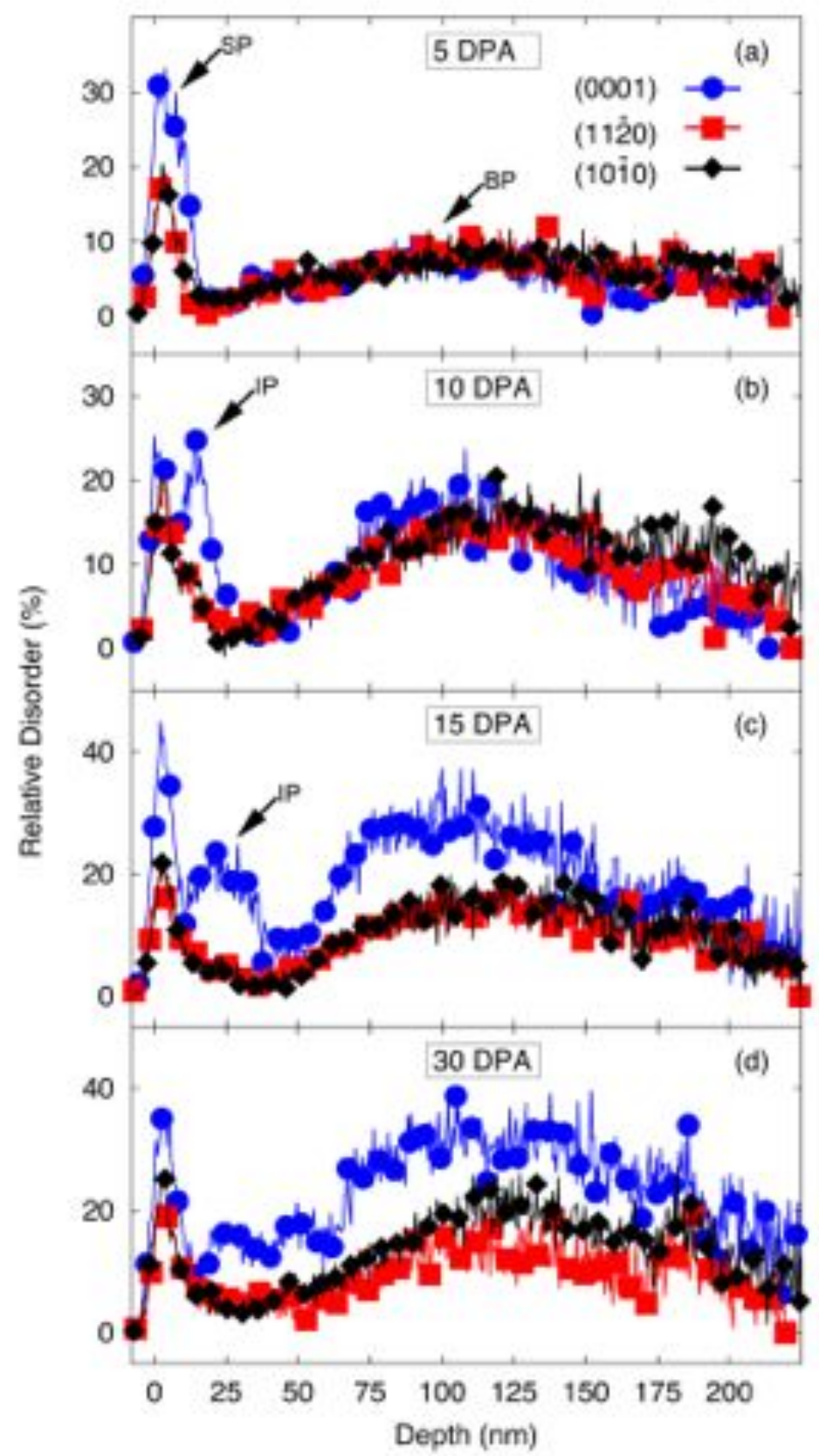

Figure 4.12: (a-d) Depth profiles of relative disorder measured by RBS/C in (0001), $(11 \overline{2} 0)$, and (1010) $\mathrm{ZnO}$ to increasing DPA. Bombardment was performed at RT with $500 \mathrm{keV} \mathrm{Xe}$ ions at $7^{\circ}$ off the [0001], [112̄0], and [10̄0] directions, respectively. For clarity, every 15th experimental point is depicted. The legend in (a) applies to all four panels. 
Figures 4.12(a-d) show experimentally measured profiles of relative disorder in

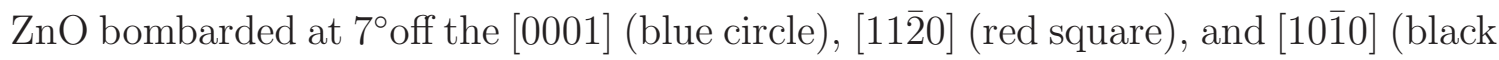
diamond) directions to 5, 10, 15 and 30 DPA, respectively. The spectra shown in Fig. 4.12(a) shows clearly resolved surface peaks (SP) and bulk peaks (BP) of disorder for all three surface terminations (marked by black arrows). Fig. 4.12(a) reveals that disorder levels in the SP of both non-polar terminations [(11 $\overline{2} 0)$ and $(10 \overline{1} 0)]$ are nearly identical $(\sim 20 \%)$ compared to $\sim 32 \%$ disorder in polar (0001) at 5 DPA. The SP of the polar surface termination (0001) is also broadened, extending over a larger region. This is in contrast to the virgin spectra (not shown) of unirradiated samples which show that all three surface terminations have similar disorder levels and widths in the SP $(\sim 9-12 \%)$.

Fig. 4.12(a) also reveals that damage levels in the BP are nearly indistinguishable at $5 \mathrm{DPA}$ for all three samples. Additionally, the BPs are situated substantially deeper than the TRIM predicted location of the maximum nuclear energy loss $\left(R_{p d}\right.$ $\sim 68 \mathrm{~nm})$. The shift of the BP relative to the $R_{p d}$ has previously been attributed to end of range effects due to an excess of ballistically interstitials [13]. This explanation is consistent with the previous results which show that the BP shifts to a depth where the profiles of excess interstitials have a maxima [11]. A shifting of the BP is typical for materials with metallic or largely ionic bonding [32] that exhibit strong DA at RT [2].

Fig. 4.12(b) reveals an interesting departure from the TRIM predicted profiles of lattice vacancies for (0001) ZnO irradiated to 5 DPA, which has been reported previously [11]. An intermediate peak develops which is positioned between the SP and BP (marked by an arrow) centered $\sim 13 \mathrm{~nm}$ from the surface. The formation of this feature is accompanied by a reduction in the intensity of the SP for the (0001) surface termination as compared to the 5 DPA sample (Fig. 4.12(a)). In 


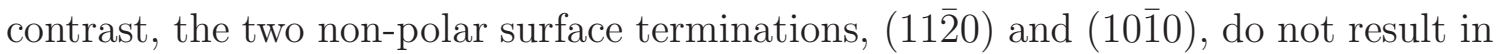
the formation of a detectable IP. A doubling of the fluence from 5 to 10 DPA has also resulted in essentially no measurable change in the SP intensity for both of the nonpolar terminations. Additionally, the BP for (0001) surface termination is positioned

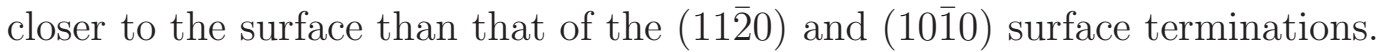

Fig. 4.12(c) reveals that with continued irradiation to 15 DPA, the IP in (0001) has broadened and shifted to deeper depths, $\sim 25 \mathrm{~nm}$ from the surface. The SP intensity for (0001) has roughly doubled, the BP has substantially broadened, and an abrupt step in the ion-channeling spectra is observed. These findings, while unusual, have been reported previously for (0001) ZnO irradiated under the same conditions $[11,12]$. The IP and the step feature are now known to be associated with the formation of alternating layers of stoichiometric and non-stoichiometric regions [12]. More interestingly, the non-polar surface terminations do not reveal such abnormalities in the depth profiles of disorder. For the non-polar surface terminations, disorder levels in both the BP and SP have nearly saturated from their values at 10 DPA. Additionally, no discernible differences are observed in the spectra between the $(11 \overline{2} 0)$ and (1010) surface terminations, they nearly overlap over the entire depth profile.

Fig. 4.12(d) reveals that at 30 DPA, the three profiles clearly deviate from one another. The IP which was clearly resolvable in (0001) at 15 DPA no longer has the characteristics of a discernible peak. However, the step in the (0001) spectra is still present and the BP has become flatter and more broadened. The SP intensity for (0001) is reduced compared to the 15 DPA sample, in contrast to that of $(11 \overline{2} 0)$ which remains the same, and (1010) which slightly increases. Additionally, the BPs of the $(11 \overline{2} 0)$ and $(10 \overline{1} 0)$ no longer overlap as observed in the 5, 10 and 15 DPA spectra. The BP of $(10 \overline{1} 0)$ is shown to be slightly higher than that of $(11 \overline{2} 0)$, similar to the results observed in the SP for these two samples. 
Rev. 1.2

The evolution of damage buildup in the BPs and SPs of disorder for all three surface terminations is better illustrated by Figs. 4.13(a) and (b). Fig. 4.13(a) shows the fluence dependence of relative disorder in the BP for all three surface terminations. In the range of $0-10 \mathrm{DPA}$, the amount of relative disorder in the BPs for all three samples is nearly identical. At 15 DPA, the BP disorder levels clearly deviate for polar vs. non-polar surface terminations. For non-polar (11200) and (1010), disorder levels have nearly saturated from their respective values at 10 DPA. Increasing fluence levels from 15 to 30 DPA reveals only modest differences between the two non-polar surface terminations, with (1010) exhibiting slightly higher levels of relative disorder than $(11 \overline{2} 0)$. In contrast, polar (0001) has higher levels of disorder $(\sim$ $30 \%)$ at 15 DPA, as compared to the nearly saturated values of non-polar surface terminations $(\sim 20 \%)$. The disorder level in the BP for polar (0001) nearly saturates from 15 to $30 \mathrm{DPA}$, similar to non-polar samples, however the amount of relative disorder is substantially higher. One interesting point is that the clear deviation between polar and non-polar BP disorder levels occurs $~ 10$ DPA, which is approximately the fluence required to produce an IP in (0001) ZnO irradiated under the conditions used herein.

Figure 4.13(b) shows the fluence dependence of relative disorder in the SP for all three surface terminations. At 0 DPA (unirradiated) both the polar (0001) and

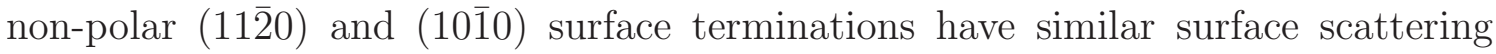
properties. For all three samples, an increase in the SP intensity is observed from 0 to 5 DPA, however (0001) clearly has an increased level of relative disorder ( $32 \%)$ as compared to the non-polar samples $(\sim 20 \%)$. For both non-polar surface terminations the SP intensity is relatively constant over the range of 5 to $30 \mathrm{DPA}$, increasing only slightly from their values at 5 DPA of $\sim 20 \%$ (for both) to $22 \%$ and $24 \%$ for $(11 \overline{2} 0)$ and $(10 \overline{1} 0)$, respectively. In contrast, the intensity of the SP 
Rev. 1.2

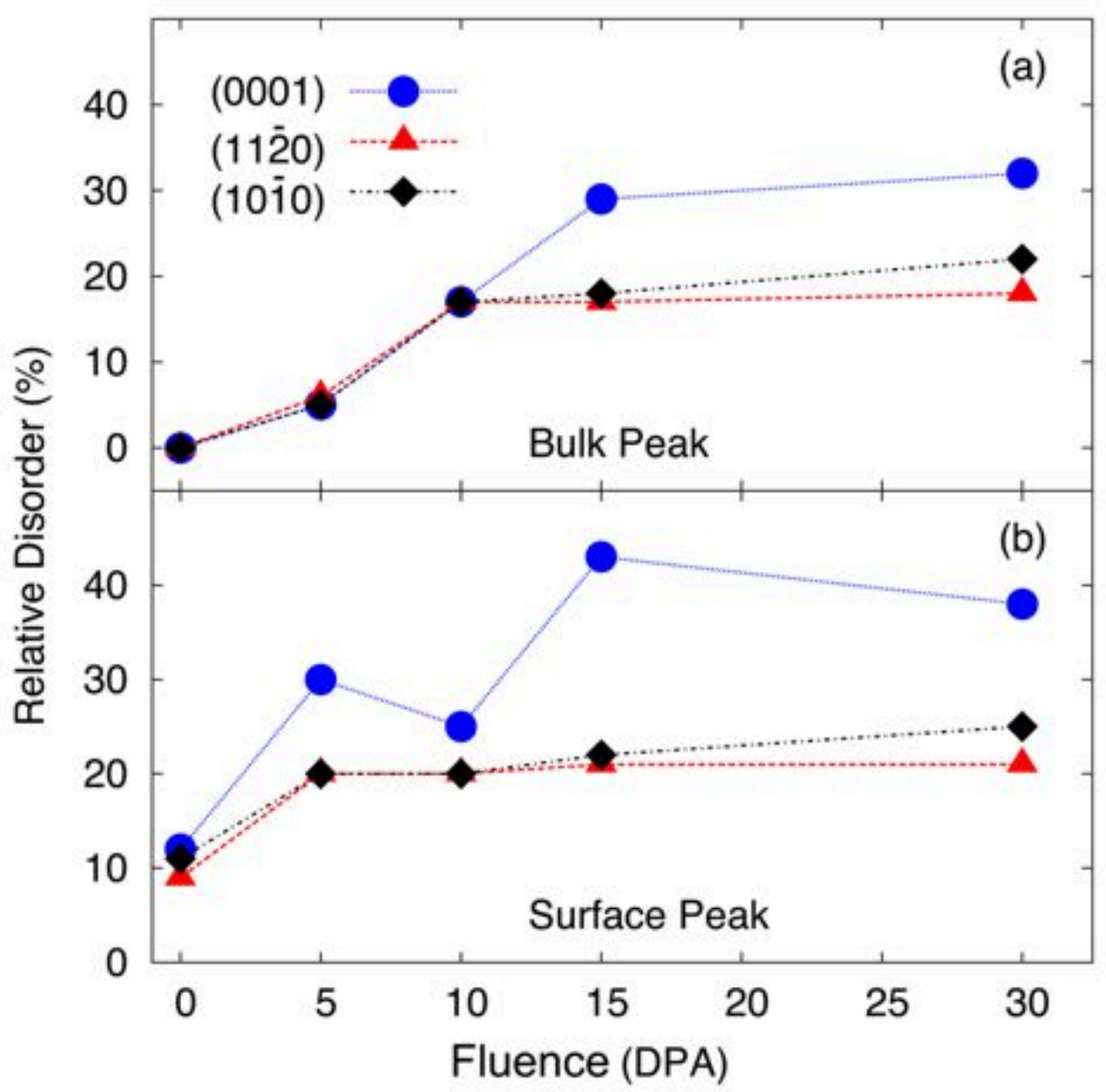

Figure 4.13: Fluence dependencies of the maximum relative disorder in (0001),

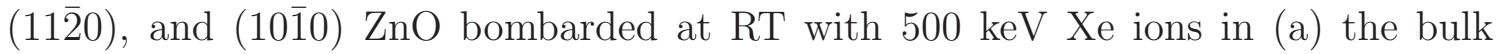
defect peak, and (b) the surface peak. The legend in (a) applies to both panels. 
for (0001) is first observed to increase from 0 to 5 DPA, followed by a decrease over the range of 5 to $10 \mathrm{DPA}$, followed by another increase from 10 to $15 \mathrm{DPA}$, where it roughly saturates $\sim 40 \%$. The intensity of the SP for (0001) appears highly dependent on the existence and position of the IP observed in Figs. 4.12(b-c). In fact, the SP intensity is observed to decrease at 10 DPA, precisely the fluence when an IP is first observed to form (or split off from) the surface. Similar SP behavior has been observed previously $y^{5,6}$, and it has been postulated that the IP might act as a defect sink, thus, reducing disorder levels at the surface [11]. As the IP moves to deeper depths with increasing fluence (away from the surface), the SP intensity of (0001) is observed to increase and finally saturate.

The results presented here are intriguing for a number of reasons. It is well known that IP formation in $\mathrm{ZnO}$ is dependent on the irradiation spectrum, occurring only for heavy (or cluster) ion irradiation $[4,6,11-13]$. However, this study clearly shows that non-polar surface terminations do not result in IP formation under heavy ion irradiation (for the conditions used herein). Clearly, the radiation response of $\mathrm{ZnO}$ is dependent on both the irradiation spectrum and the surface termination. Decoupling these two phenomena has proven to be non-trivial and a number of systematic studies have been devoted to understanding this complex behavior.

In solid crystals, damage accumulation is a competition between the damage production rate and the DA rate (post-cascade thermalization recombination). An anisotropic displacement energy could result in higher levels of disorder along certain crystallographic orientations which would result in an decrease in the damage produc-

${ }^{5} \overline{\text { Differences can be expected in the SP }}$ from Ref. [11] and the present study. This is due to enhanced near surface depth resolution in the present study by using a higher resolution detector and a decreased glancing angle of $9^{\circ}$ versus $10^{\circ}$ in Ref. [11].

${ }^{6}$ In Fig. 2 of Ref. [11], a de-convolution of the SP and IP (shown in Fig. 1) was performed, followed by a integration of the fitted SP. The trend of the areal defect concentration with increasing dose shows a similar trend to that which is observed in the present work. 
tion rate (for increased displacement energy along certain orientations). Reports of anisotropic displacement energy have been observed during high temperature ( $>400$ ${ }^{\circ} \mathrm{C}$ ) irradiation of $\mathrm{MgO}$ [107]. However, bombardment in this study was performed at RT (with no known displacement energy anisotropy) and collision cascades do not depend on crystal orientation for crystals with isotropic displacement energy. Therefore, it is more likely that DA rate anisotropy and defect diffusion play a key role in damage accumulation of polar versus non-polar $\mathrm{ZnO}$.

Enhanced radiation tolerance of one surface orientation with respect to other surface orientations has been observed in wide variety compound semiconductors [106, 108-111]. In GaN, enhanced radiation tolerance has been reported in nonpolar $A$-plane compared to $C$-plane irradiated under the same conditions [106]. The authors in that study rule out any differences in the solubility of implanted species into different orientations and suggest either enhanced DA due to the ease of point defect migration along certain crystallographic orientations (i.e. enhanced diffusion), or the stability of extended defects along certain crystallographic orientations. This is highly plausible, as diffusional and recombinational processes, as well as defect formation are likely to depend on surface orientation. Additionally, it has been observed that lattice recovery of implantation damage using thermal annealing is highly dependent on the orientation. Damage recovery occurs faster and more efficiently along certain orientations [106, 108-111]. In particular, annealing of implantation damage in $A$-plane GaN (similar wurtzite structure to $\mathrm{ZnO}$ ) appears to be much more efficient than $C$-plane GaN [106]. This observation, however enlightening, does not rule out any of the possible dominant mechanisms. Thermal annealing of ion beam induced damage will be dependent on defect diffusion and recombination rates that are dependent on the orientation and surface trapping efficiency. Additionally, the dissolution of more energetically stable extended defects may also occur at different 
rates along certain orientations, such as stacking faults on the basal plane.

Previous studies on compound semiconductors have also revealed a large dependence on the irradiation spectrum and the level of disorder $[55,56]$. It has been proposed that the large ionization per unit damage associated with light ions may act to inhibit defect cluster nucleation [59]. It has also been shown that ionizing radiation, using electrons, with a knock-on energy below the displacement energy threshold, can promote the recovery of isolated amorphous regions in defective crystals [60]. In compound semiconductors, there is strong evidence supporting the existence of defects with varied charged states [112] with the resultant defect charge state being strongly correlated to the irradiation spectrum [113]. To explain the complex defect dynamics in such systems, it has been suggested that ionized point defects in semiconductors and insulators may have a lower barrier to migration than that of their non-ionized counterparts. This process is known as ionization enhanced diffusion (IED) [57, 58]. The formation, stability and diffusion of multiple defect charge states, dependent on the irradiation spectrum, coupled with a polar termination that exhibits a net surface charge may act to substantially alter defect diffusion. In partially damaged non-amorphizable crystals, where solid phase epitaxial recrystallization models do not apply, lattice recovery during irradiation occurs through defect diffusion and annihilation. Such processes are likely to depend, not only on the defect charge state, but also on the crystallographic orientation and the diffusional pathways to the free surface. Additionally the trapping efficiency at the surface may be highly dependent on the net charge on the surface and the dominant defect charge state interacting with the surface.

Both of these effects, DA anisotropy and ionization enhanced diffusion, provide possible explanations for the phenomena which is observed in ZnO. Cascade density effects (light ion versus heavy ion) have been reported in $\mathrm{ZnO}$ [4]. The charge state, 
stability and diffusivity of defects nucleated out of dense cascades may differ substantially from that of light ion irradiation. Higher charge state defects may experience an enhanced rate of diffusion toward the polar surface due to the electrostatic potential present at the sample surface. This may explain differences observed in heavy ion irradiated polar and non-polar surface terminated $\mathrm{ZnO}$, however, more experiments are clearly needed to fully explain such complex behavior.

The results of this work could be summarized as follows.

(i) The polar $\mathrm{ZnO}$ surface strongly influences damage buildup in the first $\sim 50 \mathrm{~nm}$ under heavy ion bombardment.

(ii) Non-polar ZnO surfaces exhibit enhanced radiation tolerance as compared to polar surface terminations.

(iii) The formation of an IP in non-polar orientations is completely suppressed and damage accumulation in the bulk and surface are substantially reduced compared to polar surface terminations.

(iv) Given the fact that IP formation is dependent upon heavy ions, it is plausible that an ionization enhanced diffusion model coupled with a charged polar surface are responsible for IP formation in polar $\mathrm{ZnO}$.

4.6 Comparing the effect of surface modification in polar and non-polar $\mathrm{ZnO}$ bombarded with heavy ions

Bombardment of polar (0001) ZnO crystals with heavy ions results in highly unusual damage buildup, including an additional intermediate peak (IP) in damagedepth profiles measured by ion channeling, the formation of near-surface nanocavities, and stoichiometric imbalance. Recent reports have shown that surface modification of (0001) ZnO before bombardment can substantially reduce IP intensity, suppress cavity formation, and reduce disorder levels in the bulk. Additionally, it has 
also been demonstrated that DA is substantially higher, with a noticeable absence of an IP, in non-polar terminated $\mathrm{ZnO}$ bombarded with heavy ions. Here, we use high resolution RBS/C to show that non-polar [(1120) and (1010)] surface terminations of $\mathrm{ZnO}$ which have undergone a surface modification before being bombarded with 500 $\mathrm{keV} \mathrm{Xe}$ ions do not exhibit the same drastic reduction in disorder levels observed in polar (0001) $\mathrm{ZnO}$. The results presented show that while surface modification may act to suppress radiation damage in (0001) $\mathrm{ZnO}$, the effect is not present in polar surface terminated samples (for conditions used herein).

$\mathrm{ZnO}$ is a transparent, non-toxic, wide band gap $(\sim 3.4 \mathrm{eV}$ at $\mathrm{RT})$ semiconductor from the II-VI family [1]. The chemical and physical properties of $\mathrm{ZnO}$ are highly attractive for a number of applications such as photonic, high-frequency, and hightemperature/high-power electronic devices [1]. The fabrication of $\mathrm{ZnO}$ based devices will likely require processing techniques, such as ion implantation for doping, etching, metallization and isolation. Despite the observation that $\mathrm{ZnO}$ is more radiation tolerant than GaN [13], the difficulty associated with reliable p-type doping and the complexity of defect accumulation have hindered its potential to compete for blue-UV light emitters $[1,2]$.

The study of defects in $\mathrm{ZnO}$, their accumulation during ion implantation, and their their role on structural, electronic, and optical properties are widely researched. A number of studies have focused on understanding radiation induced defect accumulation in $\mathrm{ZnO}[1-8,11-13]$. For light ion irradiation, it has been shown that $\mathrm{ZnO}$ is non-amorphizable while it remains stoichiometric and damage accumulation is readily predictable.

Reducing levels of post implantation disorder is highly desirable, hence several studies utilizing light ions have focused on methods to mitigate radiation induced defects, both in-situ by performing implantation at elevated temperatures, and post- 
implantation thermal annealing $[9,90,115]$. However, the situation is more complicated under heavy- or cluster-ion irradiation. In those cases, damage accumulation is highly anomalous with the presence of an IP in depth profiles of disorder measured by ion channeling $[4,6,11-13]$. It is now known that IP formation is associated with stoichiometric imbalance and the presence of surface cavities [11, 12].

One method to reduce damage accumulation (and IP formation) in heavy ion irradiated (0001) $\mathrm{ZnO}$ has recently been reported [116]. It was shown that cavity formation and stoichiometric imbalance can be mitigated by depositing a pre-implantation

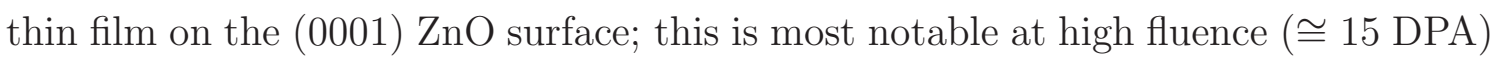
where it serves to suppress the IP [116].

Reduced ion beam damage buildup has also been reported for non-polar $\mathrm{ZnO}$ and GaN in the form of enhanced DA $[106,117]$. Such DA differences have been reported for other compound semiconductors and are observed experimentally as a reduced level of disorder when non-polar orientations are bombarded relative to the polar orientation (with the same irradiation conditions) [107-111].

The desire to understand radiation damage buildup in such non-polar $\mathrm{ZnO}$ and GaN is driven by the observation of the quantum-confined Stark effect (QCSE) in devices grown on polar substrates [97-99]. The lack of an inversion center in the wurtzite structure results in the formation of a polar surface along the $C$-axis (0001) [61]. Cleaving $\mathrm{ZnO}$ along this direction results in a strong piezoelectric and spontaneous polarization leading to a built-in electric field. Polarization effects have profound implications on devices which causes a spatial separation of the electron and hole wave functions, restricts carrier recombination efficiency, and reduces oscillator strength. [100] For the case of GaN-based LEDs, the internal quantum efficiency has been shown to increase to $50 \%$ by growing the LED on non-polar substrates [105].

Interestingly, the IP that appears in heavy ion-irradiated polar (0001) ZnO does 
not form in non-polar [(1120) and (1010)] surfaces irradiated to the same conditions [117]. The amount of stable disorder both at the sample surface and in the bulk (at depths close to the maximum nuclear energy loss) for non-polar surface terminated samples is dramatically reduced compared to their polar counterpart. Additionally, damage accumulation saturates (both surface and bulk) at substantially lower DPA.

The observation of enhanced DA of non-polar [(112̄0) and (1010)] ZnO coupled with the previous report that a thin film placed on the polar (0001) surface dramatically reduces ion beam damage buildup is intriguing. Here, we simultaneously bombard $\mathrm{ZnO}$ crystals (both polar and non-polar orientations, with and without $\mathrm{Al}_{2} \mathrm{O}_{3}$ surface modification) with $500 \mathrm{keV}$ Xe ions and observe that, although surface modification completely suppresses the formation of an IP in polar $\mathrm{ZnO}$ and reduces disorder in the bulk, it has a negligible effect on bulk radiation damage buildup properties in non-polar films. Disorder levels at the $\mathrm{ZnO} / \mathrm{Al}_{2} \mathrm{O}_{3}$ interface are greater for surface modified non-polar films (being higher than unmodified samples), however, at depths just below the $\mathrm{ZnO} / \mathrm{Al}_{2} \mathrm{O}_{3}$ interface, disorder levels are reduced for surface modified samples compared to those which were unmodified.

Wurtzite $\mathrm{ZnO}$ single crystals (oriented and polished) of three different facets (0001), (112̄0), and (1010), also referred to as $C$-, $A$-, and $M$-plane, respectively, were obtained from MTI Corp. Selected crystals were coated with an $\sim 5 \mathrm{~nm}$ thick conformal layer of $\mathrm{Al}_{2} \mathrm{O}_{3}$ by alternating exposures to trimethyl-aluminum (TMA) and water precursors in a warm wall atomic layer deposition (ALD) reactor (Kurt J. Lesker), as described elsewhere [91]. During the deposition, reactor walls and the sample stage were kept at $125{ }^{\circ} \mathrm{C}$, while the sample was repeatedly exposed to the following sequence: TMA pulse, Ar purge, $\mathrm{H}_{2} \mathrm{O}$ pulse, and Ar purge. The pressure during TMA and $\mathrm{H}_{2} \mathrm{O}$ pulses was 0.8 Torr and 1.2 Torr, respectively.

The $4 \mathrm{MV}$ ion accelerator (National Electrostatics Corporation, model 4UH) at 
Lawrence Livermore National Laboratory was used for both ion irradiation and ion beam analysis. Bombardment was performed at RT with $500 \mathrm{keV} \mathrm{Xe}{ }^{+}$ions at $7^{\circ}$ off

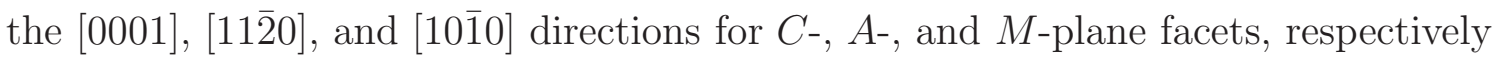
for both bare (i.e., uncoated) samples and those coated with $\mathrm{Al}_{2} \mathrm{O}_{3}$ by ALD. Irradiation was performed to $15 \mathrm{DPA}$, which corresponds to a Xe ion fluence of $3 \times 10^{15}$ with beam flux values $\left(\sim 1 \times 10^{12} \mathrm{~cm}^{-2} \mathrm{~s}^{-1}\right)$ kept constant to maintain a constant displacement generation rate of $\sim 7 \times 10^{-3} \mathrm{DPA} / \mathrm{s}$ at a depth of the maximum nuclear energy loss $\left(R_{p d} \cong 68 \mathrm{~nm}\right)$. The DPA values are quoted as the concentration of ion-beam-generated lattice vacancies at the $R_{p d}$ normalized to the atomic concentration of $\mathrm{ZnO}\left(8.3 \times 10^{22}\right.$ atoms $\left.\mathrm{cm}^{-3}\right)$ and were calculated with the TRIM code (version SRIM-2008.04) [20] with threshold energies for atomic displacements of 34 and $44 \mathrm{eV}$ for $\mathrm{Zn}$ and $\mathrm{O}$ sublattices, respectively [69].

Implantation-induced disorder was measured by RBS/C with $2 \mathrm{MeV}^{4} \mathrm{He}^{+}$ions incident along the [0001], [11̄20], and [10̄10] directions for $C$-, $A$-, and $M$-plane samples, respectively. The probing beam was backscattered into a detector at $99^{\circ}$ relative to the incident beam direction, providing enhanced near-surface depth resolution. All RBS/C spectra were analyzed with a conventional algorithm [70] for extracting the effective number of scattering centers (referred to below as "relative disorder").

Figure 4.14(a-c) show experimentally measured profiles of relative disorder in $\mathrm{ZnO}$ bombarded at $7^{\circ}$ off the [0001], [112̄0], and [1010] directions respectively. Bombardment was performed to 15 DPA with $500 \mathrm{keV}$ Xe ions for samples both with an $\mathrm{Al}_{2} \mathrm{O}_{3}$ layer (blue circle) and unmodified $\mathrm{ZnO}$ (red square), labeled "coated" and "bare", respectively.

Both spectra, shown in Fig. 4.14(a), show clearly resolved surface peaks (SP) and bulk peaks (BP) of disorder (marked by black arrows). The bare spectra clearly deviates from TRIM predicted profile of ballistically generated vacancies [11]. It 
Rev. 1.2

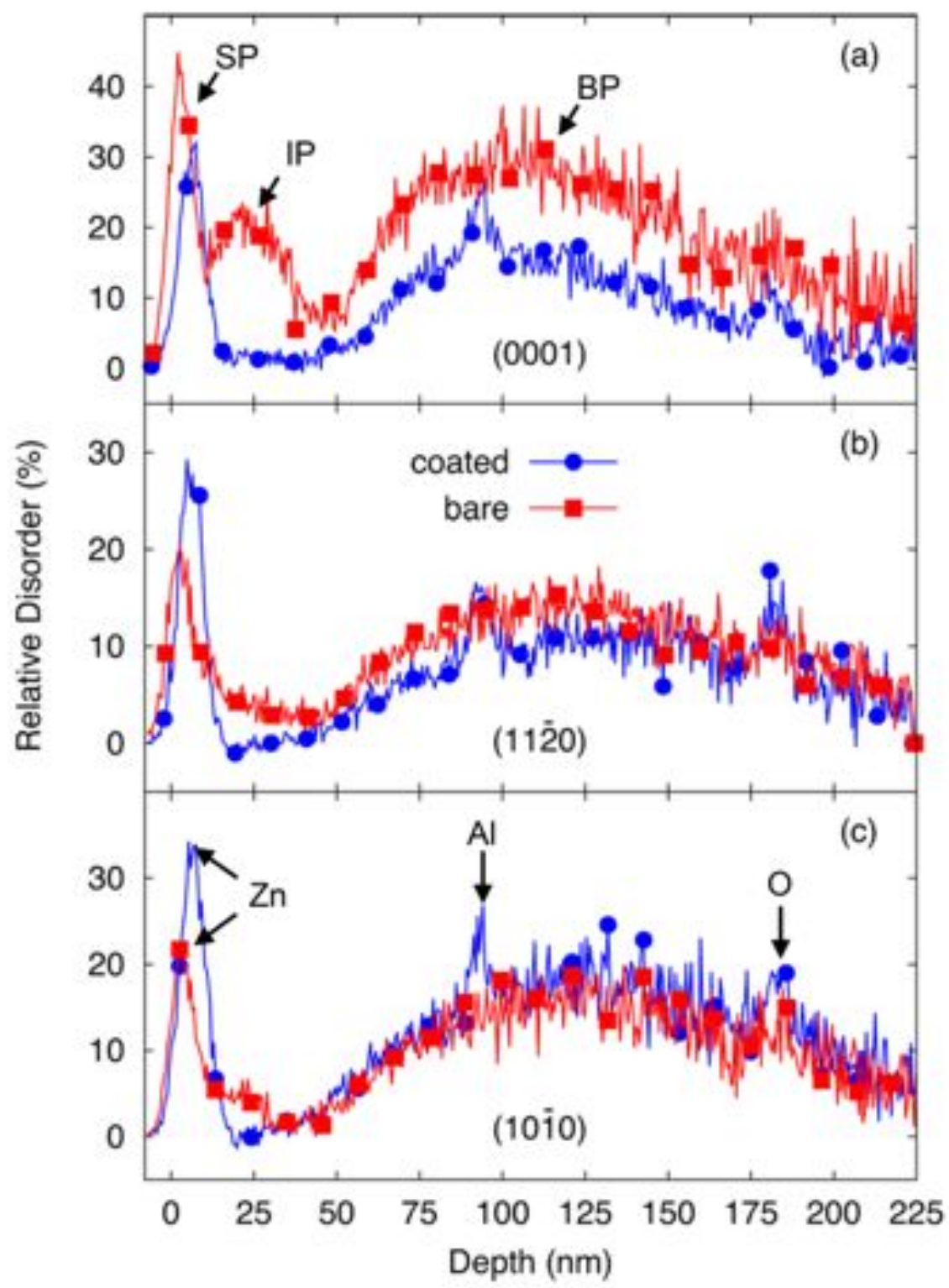

Figure 4.14: Depth profiles of relative disorder measured by RBS/C in (a) $C$-plane (0001), (b) $A$-plane (11̄20), and (c) $M$-plane (1010) ZnO irradiated to 15 DPA for both bare (i.e. uncoated) and $\mathrm{Al}_{2} \mathrm{O}_{3}$ surface modified (coated) samples. In (a-c) bombardment was performed at RT with $500 \mathrm{keV}$ Xe ions at $7^{\circ}$ off the [0001], [112̄0], and $[10 \overline{1} 0]$ directions, respectively. The zinc $(\mathrm{Zn})$, oxygen $(\mathrm{O})$, and aluminum (Al) peaks, marked by vertical arrows in (c), and the the legend in (c) apply to all three panels. Note, an $\mathrm{Al}$ peak is only present in coated samples. For clarity, every 20th experimental point is depicted. 
exhibits a broad IP positioned between the expected SP and BP of disorder centered $\sim 25 \mathrm{~nm}$ from the surface. ${ }^{7,8}$ Additionally, the BPs are situated deeper than the TRIM predicted depth of the maximum nuclear energy loss ( $R_{p d} \sim 68 \mathrm{~nm}$ ). The shift of the BP relative to the $R_{p d}$ has described in detail elsewhere [11-13].

In contrast, the coated sample [in Fig. 4.14(a)] does not exhibit an IP and the SP and BP intensity are substantially reduced (when compared to the coated sample). This effect is similar to a recent report using a larger implantation angle (i.e. shallower vacancy generation profile), however in the present study, the IP is completely suppressed [116]. ${ }^{9}$ The reduction in relative disorder in the BP extends to depths down to $\sim 200 \mathrm{~nm}$ from the sample surface. The additional peaks, most apparent in the coated samples, correspond to scattering from oxygen and aluminum [marked for reference in Fig. 4.14(c)], whereas the bare sample has an oxygen peak which is nearly indistinguishable from experimental noise.

The spectra shown in Figs. 4.14(b) and (c) show markedly different behavior than those shown in Fig. 4.14(a). The most notable feature is the lack of an IP in bare non-polar $(11 \overline{2} 0)$, and $(10 \overline{1} 0)$ spectra, a phenomena which has recently been reported [117]. Additionally, the levels of relative disorder in bare non-polar samples are substantially less than the polar samples irradiated to the same DPA $(\sim 18 \%$ versus $\sim 30 \%$, respectively) [117].

Unlike the trend observed between the two spectra of Fig. 4.14(a) where coated samples display a reduction in intensity for both SP and BP (compared to bare samples), Figs. 4.14(b) and (c) show the opposite trend for the SP and marginal

${ }^{7}$ Details of RBS/C and XTEM characterization of bare and coated (0001) ZnO samples irradiated with $500 \mathrm{keV}$ Xe ions at RT to 15 DPA at $60^{\circ}$ incident ion angle have been reported in Ref. [12] and Ref. [116], respectively.

${ }^{8} \mathrm{RBS} / \mathrm{C}$ spectra of bare (0001), (112̄0), and (1010) ZnO samples irradiated under the same conditions have been reported previously [117].

${ }^{9}$ It should be noted that the shift in the $\mathrm{Zn}$ edge to deeper depths corresponds to the fact that the actual sample surface of $\mathrm{ZnO}$ is located $\sim 5 \mathrm{~nm}$ below the actual sample surface. 


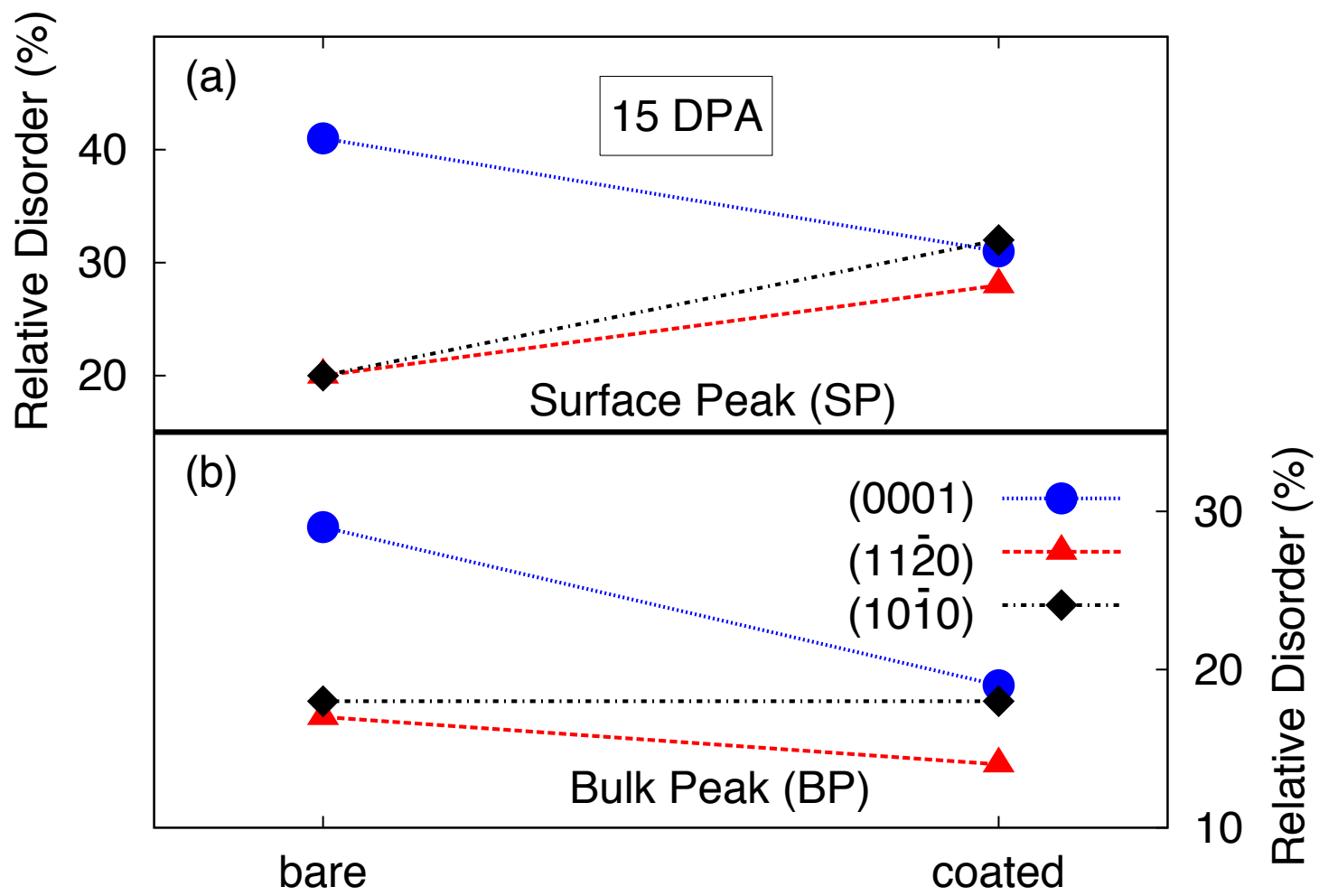

Figure 4.15: Maximum relative disorder in (a) the surface peak (SP) and (b) the

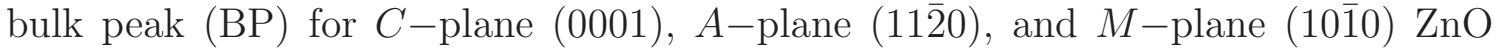
irradiated to $15 \mathrm{DPA}$ for both bare (i.e. uncoated) and $\mathrm{Al}_{2} \mathrm{O}_{3}$ surface modified (coated) samples. The values in (a) and (b) correspond to the maximum values in each of the surface and bulk peaks, respectively. Bombardment was performed at RT with $500 \mathrm{keV} \mathrm{Xe}$ ions at $7^{\circ}$ off the [0001], [1120], and [10̄10] directions, respectively. The lines connecting bare and coated data points (for each surface orientation) are depicted to clarify the trend.

[Fig. 4.14(b)] and no change [Fig. 4.14(c)] in the BP. The SPs for coated (112̄0) and (1010) samples are more intense and less broad than bare samples. Within a region $\sim 50 \mathrm{~nm}$ from the surface/interface (i.e. SP) the spectra show reduced levels of relative disorder compared to bare samples.

A comparison of bare versus coated samples irradiated to 15 DPA is better illustrated by Figs. 4.15(a) and (b) which show the maximum intensity of relative disorder (both bare and coated samples) in the SP and BP, respectively. In Fig. 4.15(a) the 
difference in SP intensity between bare polar and non-polar samples is dramatic. Polar (0001) $\mathrm{ZnO}$ exhibits $\sim 40 \%$ relative disorder compared to $\sim 20 \%$ for both non-polar $(11 \overline{2} 0)$, and (1010). Depositing a thin film of $\mathrm{Al}_{2} \mathrm{O}_{3}$ on the surface of the samples before irradiation acts to reduces the SP intensity of (0001), however for non-polar films, the SP intensity is observed to increase. The increase in SP intensity is larger for $(10 \overline{1} 0)$ compared to (1120). Interestingly, the SP intensity of all coated samples after irradiation is nearly identical $(\sim 30 \%)$.

Fig. 4.15(b) shows that, similar to the SP, the BP intensity for bare polar and non-polar samples is dramatically different. The BP intensity of (0001) is $\sim 30 \%$, whereas in both $(11 \overline{2} 0)$, and $(10 \overline{1} 0)$ the BP intensity is nearly identical $(\sim 18 \%)$. In the coated polar (0001) sample, the BP intensity undergoes a dramatic decrease, similar to the effect observed in the SP (from $\sim 30 \%$ to $18 \%$ ), similar to previous

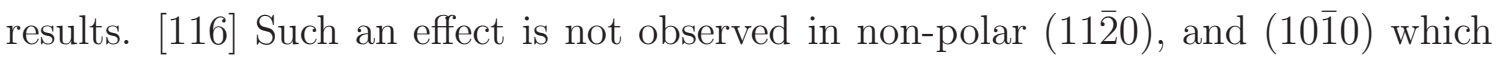
show negligible differences between bare and coated samples.

Clearly, there are large differences between polar and non-polar $\mathrm{ZnO}$ irradiated with heavy ions [116]. Previous results have shown that non-polar $\mathrm{ZnO}$ has enhanced DA properties, however the exact role of the surface (polar versus non-polar) in defect recombination efficiency and diffusion is still unknown. Enhanced radiation tolerance of one surface orientation with respect to other surface orientations has been observed in wide variety compound semiconductors, however, a meaningful explanation is still lacking [106, 108-111].

Surface modification of polar (0001) $\mathrm{ZnO}$ has been shown to be an effective method to suppress radiation damage. However, the results presented here reveal several interesting phenomena. First, given the fact that IP formation in (0001) is not completely suppressed using a shallower displacement generation profile [116], and the IP is completed suppressed in this work, suggests the efficacy of surface 
modification may be dependent on the vacancy generation profile.

Additionally, it is still unclear the exact mechanism which gives rise to the enhanced radiation properties of the surface modified polar samples. Differences between the surface modification in our previous study [117] and this one may play a role in whether or not the IP is completely suppressed. Further studies are needed aimed at tuning the surface modification to maximize the suppression of radiation damage in polar $\mathrm{ZnO}$.

It has been proposed that the presence of charged defects in $\mathrm{ZnO}$, coupled with the a (0001) polar surface may substantially alter defect diffusion [116]. It has been suggested that the ability of surface modification to reduce disorder levels could be related to a number of factors including inhibiting oxygen loss from the sample surface, peculiarities of defect diffusion in the presence of a polar surface, and mechanical stresses related to differences in the thermal expansion of $\mathrm{ZnO}$ and the thin film on the surface [117].

For non-polar $\mathrm{ZnO}$, the effect of surface modification appears negligible on the disorder levels in the bulk. Figs. 4.14(b) and (c) clearly show that at depths beyond $\sim 50 \mathrm{~nm}$ from the surface, the spectra nearly overlap (within experimental uncertainty). However, the effect of surface modification is more pronounced in the near surface region, at depths $\lesssim 50 \mathrm{~nm}$ from the surface. It is observed that surface modification increases the number of scattering centers at the sample surface, observed as an increase in the SP, however, at depths just beyond the surface, the disorder levels are observed to decrease. It has been previously suggested that the defect diffusion length in $\mathrm{ZnO}$ is $\sim 50 \mathrm{~nm}$, which agrees with our current observations [11]. This effect could be due to the defect trapping efficiency at the surface/interface which promotes defect recombination in a narrow region below the sample surface, however the current study cannot clearly identify the exact physical mechanism. 
Rev. 1.2

The results of this work could be summarized as follows.

(i) Surface modification of polar $\mathrm{ZnO}$ is an effective method to substantially reduce disorder accumulation, and in particular, suppress the formation of an IP.

(ii) Surface modification of non-polar $\mathrm{ZnO}$ has little to no observable effect on damage accumulation in the bulk.

(iii) Non-polar surfaces exhibit enhanced radiation tolerance as compared to polar surface terminations.

(iv) In both polar and non-polar $\mathrm{ZnO}$, the surface strongly influences damage buildup in the first $\sim 50 \mathrm{~nm}$ under heavy ion bombardment. Surface modification of nonpolar $\mathrm{ZnO}$ exhibits reduced levels of near surface disorder within $\sim 50 \mathrm{~nm}$ from the surface/interface. 
Rev. 1.2

\section{PULSED-ION-BEAM IRRADIATION}

Bombardment with energetic ions inevitably produces lattice disorder in crystalline targets. An energetic ion propagating through a solid creates a collision cascade along its trajectory. The ballistic formation and thermalization of the cascade occur rapidly, at time scales of up to $\sim 10^{-12}$ s. Such regimes of cascade formation and thermalization (although challenging to access experimentally) are believed to be reasonably well understood [26]. In contrast, our current understanding of the evolution of defects after cascade thermalization, which is often referred to as DA, is limited for most materials [24, 26-30]. Understanding mechanisms involved in DA is, however, highly desirable since DA plays a major role in the formation of stable post-irradiation disorder in most technologically relevant cases, including ion-beam-processing of semiconductors and radiation damage in nuclear materials $[24,27-32]$.

The present section is divided into five main subsections. The first section is a literature review of previous works on pulsed ion beam irradiation. The second section explains the physical basis for pulsed-ion-beam irradiation along with the mathematical derivations for the quantities of interest in the execution of pulsed beam experiments. The third section is devoted to a detailed description of the actual pulsed-ion-beam setup that was used in the present work. It contains information relevant to the building and operating of a pulsed beam. The final two sections are devoted to two experiments which were performed to highlight the ability of the pulsed beam method to measure the fundamental constants associated with DA, namely the time constant $[\tau$ in Section 5.4 $]$ and the diffusion length $\left[L_{\mathrm{d}}\right.$ in Section 5.5]. 
Rev. 1.2

\subsection{Introduction to pulsed-ion-beam irradiation}

The earliest investigations into pulsed-ion-beam irradiation appear in the early 1970's, not as a result of trying to understand DA directly, but were initiated while comparing two methods for delivering a spatially-uniform ion beam into metals [118]. Early studies were aimed at understanding the microstructural evolution of metals for use in a fusion reactor (a rapidly evolving field at the time). Fusion reactors pose significant challenges due to the harsh environment with combined effects from corrosive atmospheres, exceedingly large radiation fluence, mechanical stresses, high temperatures, and transmutation products (H, He, etc.). In particular, they observed significant differences between raster-scanning a beam versus delivering the fluence with a broad defocused beam [118]. Pulsed irradiation was shown to substantially reduce void sizes, as compared to raster-scanned targets, with this effect being most pronounced at high temperature. However, they also observed that pulsed irradiation led to an increase in the overall number of voids (increased density), and therefore total swelling was similar for both specimen (pulsed and raster-scanned) [118].

The pulsing that was performed in the initial work was quite fast, on the order of milliseconds, and also rather complex. Over the beam on duration, packets of $25 \mu \mathrm{s}$ sub-pulses were delivered on target. The observation of restricted cavity growth lead to further studies aimed at isolating the effect of pulsed irradiation on cavity growth. Pre-implantation was performed to generate cavities, followed by pulsed irradiation using a constant duty cycle of $50 \%[119,120]$. The results were ambiguous and no clear trend was observed. Other experiments aimed at exploring the relationship between duty cycle and the resultant microstructure [121-124]. It was generally found that for long values of the beam times on and short times off resulted in a microstructure similar to that of raster-scanned materials. However, a low fraction 
of times on and long times off restricted void growth [121-124]. Various other pulsed irradiation (single, double and triple beam) studies were performed studying the dependence of dislocation loop nucleation and growth (in binary and ternary alloys) on pulsing and temperature $[125,126]$. From this, modeling was put forth in an attempt to understand pulsed irradiation in terms of a balance between ballistically generated defects and their subsequent annealing [14, 127, 128].

Clearly, the earliest works on pulsed-ion-irradiation were dominated by the nuclear community and the driven by the desire to fabricate materials for fusion reactors. However, it was not long after this time that the semiconductor community began investigating pulsed irradiation effects. This type of pulsed beam method was used by Linnros and co-workers $[129,130]$, who measured effective time constants of $\gtrsim 1 \mathrm{~s}$ for the process of ion-beam-induced epitaxial recrystallization of Si at elevated temperatures $\left(200-300{ }^{\circ} \mathrm{C}\right)$. In another pulsed beam study, an estimate was placed on divacancy activation energy [131], and it was suggested that divacancy production and diffusion governed defect kinetics at high fluence in silicon. Carter et. al, earlier, proposed that the nature of the defects not only depends on the fluence, but also depends directly on the stable defect lifetime [40-42], and could be described in terms of competitive annealing processes.

Due to the overwhelming number of electronic devices comprised of silicon, it is arguably one of the most extensively studied materials. This is certainly the case for radiation effects in $\mathrm{Si}$, for which a wide body of literature exists. During device fabrication and processing, ion implantation methods are employed to introduce dopants and perform isolation. For this fact, the literature pertaining to defect dynamics, damage production and annealing as a result of ion implantation in silicon is immense [132].

During ion irradiation, ion-solid interactions induce structural transformations. 
This is due to energy being ballistically transferred from impinging ions to target atoms and recoils. Annealing studies have shown that there exists a hierarchy of defect structures that are dominant in Si. Each stable defect structure persists as dominant within various temperature regimes. For Si at RT and below, single interstitials and vacancies are are active, and readily diffuse [36]. At elevated temperatures $\left(100-250^{\circ} \mathrm{C}\right)$ and higher defect concentrations, di-vacancies and di-interstitials become dominant [133]. Due to the nature of various dynamic defect annealing phenomena that are active at RT, binary collision approximation (BCA) models often over predict the amount of stable post implantation disorder.

Making accurate predictions of post implantation disorder is exceedingly challenging. Many of the phenomena that govern damage accumulation are difficult to model [134], and accurate theoretical predictions are mediocre at best. As fluence levels increase, typically, so does the amount of disorder and amorphization can occur. This process has been shown to be highly non-linear [35]. In the low fluence regime, pre-existing defect structures act as undersaturated defect sinks, readily annihilating Frenkel pairs which results in sub-linear behavior [36]. After exceeding a threshold fluence, a rapid super-linear increase in disorder is observed, followed by saturation [132]. The super-linearity, in part, has been attributed to a reduction in the threshold energy for atomic displacements in a pre-damaged crystal. Therefore, the concentration of stable defects is proportional to the concentration of existing defects, a so-called sensitization process [36].

In an attempt to describe the amorphization process in silicon, a damage cascade overlap model has been proposed [33, 34]. In this model, it has been suggested that isolated amorphous zones occur along ion tracks that, upon continued bombardment, begin to partially overlap forming permanently damaged zones. The theory predicts increased disorder when the vacancy concentration outside the collision cas- 
cade tracks is on the same order of magnitude as the vacancy concentration in the bulk (i.e highly overlapped damaged zones). This model distinguishes two distinct annealing time scales, one that is independent of temperature, that is initial cascade collapse $\left(<10^{-12} \mathrm{~s}\right)$, and another that depends strongly on temperature and occurs over much longer time scales [33]. Above a critical threshold damage level, direct amorphization (Crowder/Gibbons overlap) appears to dominant stable lattice disorder.

Additionally, silicon has also been shown to exhibit a strong flux effect at RT [40, 42, 43]. A flux effect is observed when defect stabilization time $\tau$ is comparable to the average time interval between the formation of spatially overlapping damage zones originating from different collision cascades. At very low fluence, close proximity Frenkel pairs tend to annihilate and flux effects are rarely observed experimentally. However, with increasing fluence, the flux effect is more pronounced as stable defect complexes form which inhibit defect diffusion and annihilation. Estimates of the time constants of DA $(\tau)$ have typically involved an analysis of the flux effect [39, $43,135,136]$. However, the flux effect convolutes both temporal $(\tau)$ and spatial $\left(L_{d}\right)$ information that must be separated in order to obtain $\tau$. Such estimates of $L_{d}$ (and, hence, the extraction of $\tau$ from the experimental flux dependence of disorder) require serious assumptions about the explicit defect interaction processes [39, 135-138].

This is where the utility of the pulsed-ion-beam method is fully realized, and is elaborated upon in further sections. Spatial and temporal information can be separated in experiments using pulsed ion beams which allows for measurement of both $\tau$ and $L_{d}$. Such measurements can substantially clarify previous estimates of these quantities and aid in understanding the physical processes involved in DA. 


\subsection{Parameters and model}

Figure 5.1(a), shows a schematic representation of pulsed-ion-beam irradiation. By having precise control over $t_{o n}$, and the maximum flux, $\phi_{\max }$, a train of pulses can be delivered that each have an associated fluence per pulse, given by

$$
\Phi_{\text {pulse }}=\phi_{\text {max }} \cdot t_{\text {on }} .
$$

The number of pulses can be defined as

$$
N_{p}=\frac{\Phi_{\text {total }}}{\Phi_{\text {pulse }}},
$$

where $N_{p}$ is the number of pulses, $\Phi_{\text {total }}$ is the total accumulated fluence, and $\Phi_{\text {pulse }}$, again, is the fluence per pulse, as defined by Eq. 5.1.

Figure 5.1(b) is a diagram of one possible damage buildup scenario in a sample due to pulsed-ion-beam irradiation which shows the amount of stable lattice disorder (after initial cascade quenching) during a cycle. While the beam is pulsed on-target, during a time $t_{o n}$, differential damage is assumed to increase linearly proportional to a constant rate, $\alpha$ (i.e. it is assumed that there is no annealing during $t_{o n}$ ). The overall defect concentration, $n_{d}$ after a duration $t_{o n}$ is simply the product,

$$
n_{0} \equiv n_{d}\left(t_{o n}\right)=\alpha \cdot t_{o n},
$$

where $n_{d}$ is the defect concentration, $n_{0}$ is the maximum defect concentration after a pulse duration of $t_{o n}$.

As the beam is pulsed off-target, it is suggested that the defect concentration will decrease via dynamic defect annealing in a way that is proportional to a defect stabilization time, $\tau$, and governed by the concentration of defects [40-42]. After a 
Rev. 1.2

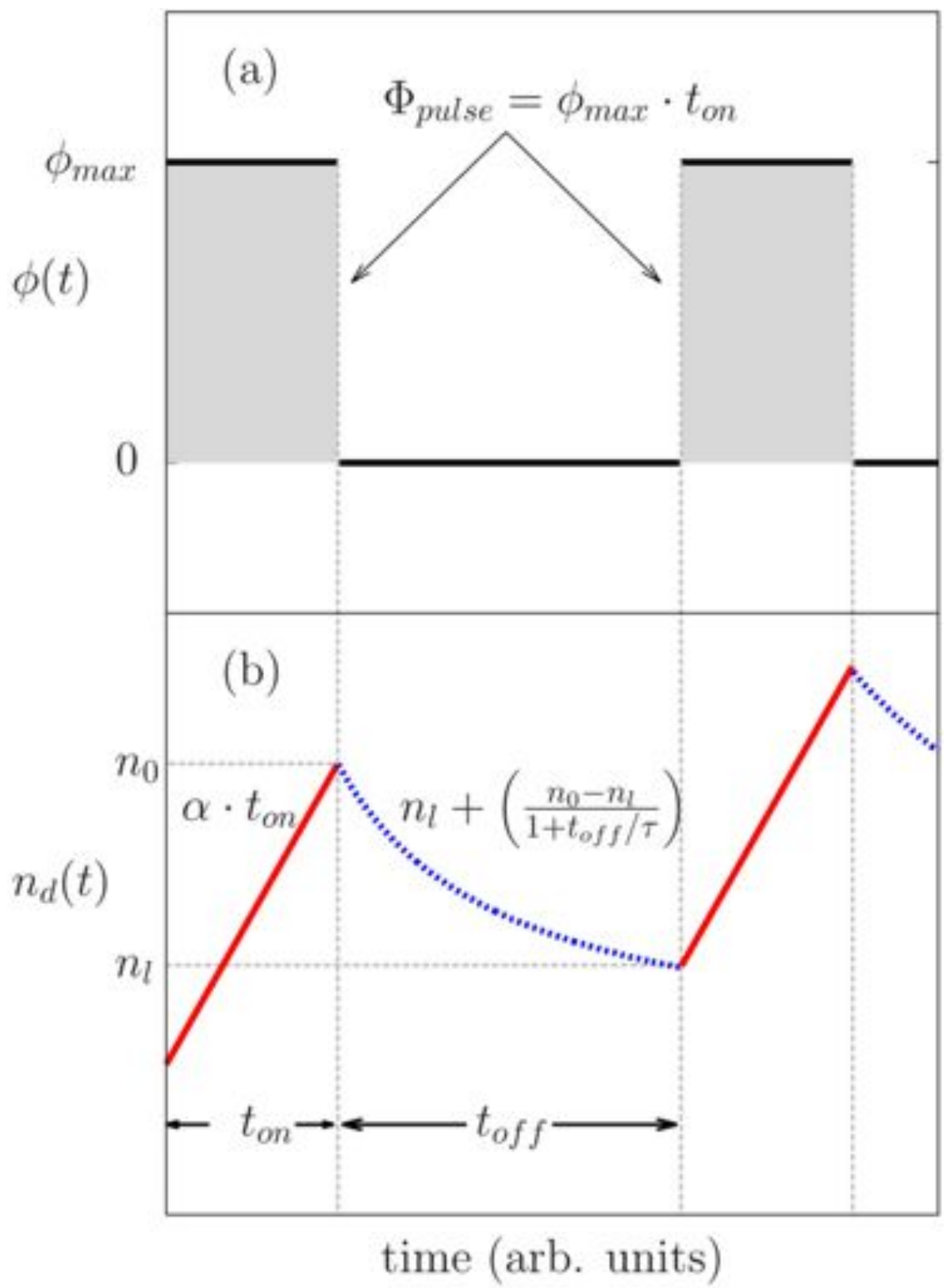

Figure 5.1: Schematic representation of the pulsed ion beam experiment. (a) The fluence per pulse, $\Phi_{\text {pulse }}$, is $t_{o n} \cdot \phi_{\max }$. (b) Linear defect production during $t_{o n}$ and dynamic defect annealing during $t_{o f f}$ are depicted. 
single train of pulses, both on- and off-target, the defect concentration per pulse will be proportional to the linear buildup, and the subsequent annihilation, given by

$$
n_{d}^{\text {pulse }}\left(t_{o n}+t_{o f f}\right)=n_{d}\left(t_{o n}\right)-n_{d}\left(t_{o f f}\right) .
$$

The defect evolution could be expressed by the following equation:

$$
\frac{\partial}{\partial t} n_{d}(t)=\alpha-\frac{1}{\tau} n_{d}(t)^{\beta},
$$

where $n_{d}(t)$ is the defect concentration, $\alpha$ is the linear buildup, $\tau$ is the defect stabilization time, and $\beta$ governs the order of the defect kinetics. By making precise measurements, first to determine the dominating order of the kinetics, then by an accurate measurement of the defect stabilization time through fitting, information related to dynamic defect annealing can be revealed.

For example, if defect accumulation is governed by simple interactions of point defects, then the dependence on the stable lattice disorder after a single train of pulses would be exponential, indicative of first order processes. This would most likely be the scenario for low fluence levels. For higher fluence, more complex defect structures will form through agglomeration or diffusion. These higher order defect structures will alter the order of the defect kinetics to reflect the primary interaction type. The present discussion is limited to second order, however, one could envision a system where defect interactions at even higher fluence are dominated by vacancy clusters, extended defects, etc.

Under the assumption that defect kinetics are governed by second order $(\beta=$ 2) reactions (di-vacancy/di-insterstitial) an analytical form of the dynamic defect annealing can be determined. This comes as a solution to Eq. 5.5 assuming no defect production. This is a reasonable assumption in that the beam is no longer 
illuminating the target, and initial cascade collapse would be much faster than the subsequent cascade thermalization processes that are to be measured. In light of this, the solution is given by

$$
n_{d}\left(t_{o f f}\right)=n_{l}+\frac{n_{0}-n_{l}}{1+\frac{t_{o f f}}{\tau}}
$$

where $n_{0}$ is the maximum defect concentration per pulse, $n_{l}$ is the final annealed defect concentration, $\tau$ is the defect stabilization time, $t_{\text {off }}$ is the annealing time after the initial pulse, and $n_{d}$ is the defect concentration in time.

We can use Eq. 5.3 and Eq. 5.6, plug them into Eq. 5.4, and apply the appropriate boundary conditions to write a balance equation to describe the concentration of defects at any later time as

$$
n_{d}^{p u l s e}=\alpha t_{o n}-\left(n_{l}-\frac{n_{l}}{1+\frac{t_{\text {off }}}{\tau}}\right) .
$$

Figure 5.1(b) and Eq. 5.7 also show that after a single pulse, the amount of annealing is limited by the ratio of $t_{\text {off }}$ to $\tau$. However, in the limit of $t_{\text {off }} \gg \tau$, there clearly exists a lower bound limit to the amount of DA can take place after the beam has been extinguished. Due to this fact, the relative efficiency for DA can be defined as

$$
\gamma=\frac{n_{0}-n_{l}}{n_{0}}
$$

where $\gamma$ is the DA efficiency, $n_{0}$ is the maximum defect concentration per pulse, and $n_{l}$ is the final annealed defect concentration.

Figure 5.2 shows a schematic of the number of stable lattice defects, $N_{d}$, after irradiation, as a function of increasing $t_{\text {on }}$. This is applicable for the case of $\phi_{\max }$ and 
$t_{\text {off }}$ fixed, with $t_{\text {off }} \gg \tau$. As seen in Fig. 5.2, three regimes, labeled as $I, I I$, and $I I I$, that represent the cases of $t_{o n}<\tau, t_{o n} \sim \tau$ and $t_{o n}>\tau$, respectively. For region $I$, for small $t_{o n}$, the relative disorder, $N_{d}$ will be very low. This is due to the vast majority of ballistically generated defects being being annealed out after every pulse. In the limit of $t_{\text {off }} \gg \tau$, where $\gamma$ is essentially a maximum, the relative disorder can easily be predicted, and clearly this value should be independent of $\tau$.

The relative disorder can be defined in terms of previously defined quantities. The overall relative disorder should be the product of the concentration of defects per pulse (both created and annealed), and the total number of pulses. Combining these terms from Eqs. 5.1 and 5.2 gives

$$
N_{d}=n_{d}^{p u l s e} \cdot N_{p}=n_{d}^{\text {pulse }}\left(\frac{\Phi_{\text {total }}}{\phi_{\max } \cdot t_{\text {on }}}\right)
$$

where $N_{d}$, again, is the relative disorder, $n_{d}^{\text {pulse }}$ is the concentration of defects per pulse, $\Phi_{\text {total }}$ is the total accumulated fluence, $\phi_{\max }$ is the maximum flux, and $t_{o n}$ is the pulse on duration. Now substituting into Eq. 5.9 the expression for $n_{d}^{\text {pulse }}$ yields

$$
N_{d}=\frac{\Phi_{\text {total }}}{\phi_{\text {max }} \cdot t_{o n}} \cdot\left(\alpha t_{o n}-\left(n_{l}-\frac{n_{l}}{1+\frac{t_{o f f}}{\tau}}\right)\right) .
$$

In the limit of $t_{o f f} \gg \tau$, Eq. 5.10 simplifies to

$$
N_{d}=\frac{\Phi_{\text {total }}}{\phi_{\max } \cdot t_{o n}}\left(\alpha \cdot t_{o n}-n_{l}\right)
$$

where the final annealed defect concentration, $n_{l}$, has been defined in terms of $\gamma$ through Eq. 5.8. In addition, $n_{0}$, the maximum defect concentration per pulse has also been defined, as seen in Eq. 5.3. In light of this, the minimum amount of relative disorder expected in the aforementioned limits, can be written as 
Rev. 1.2

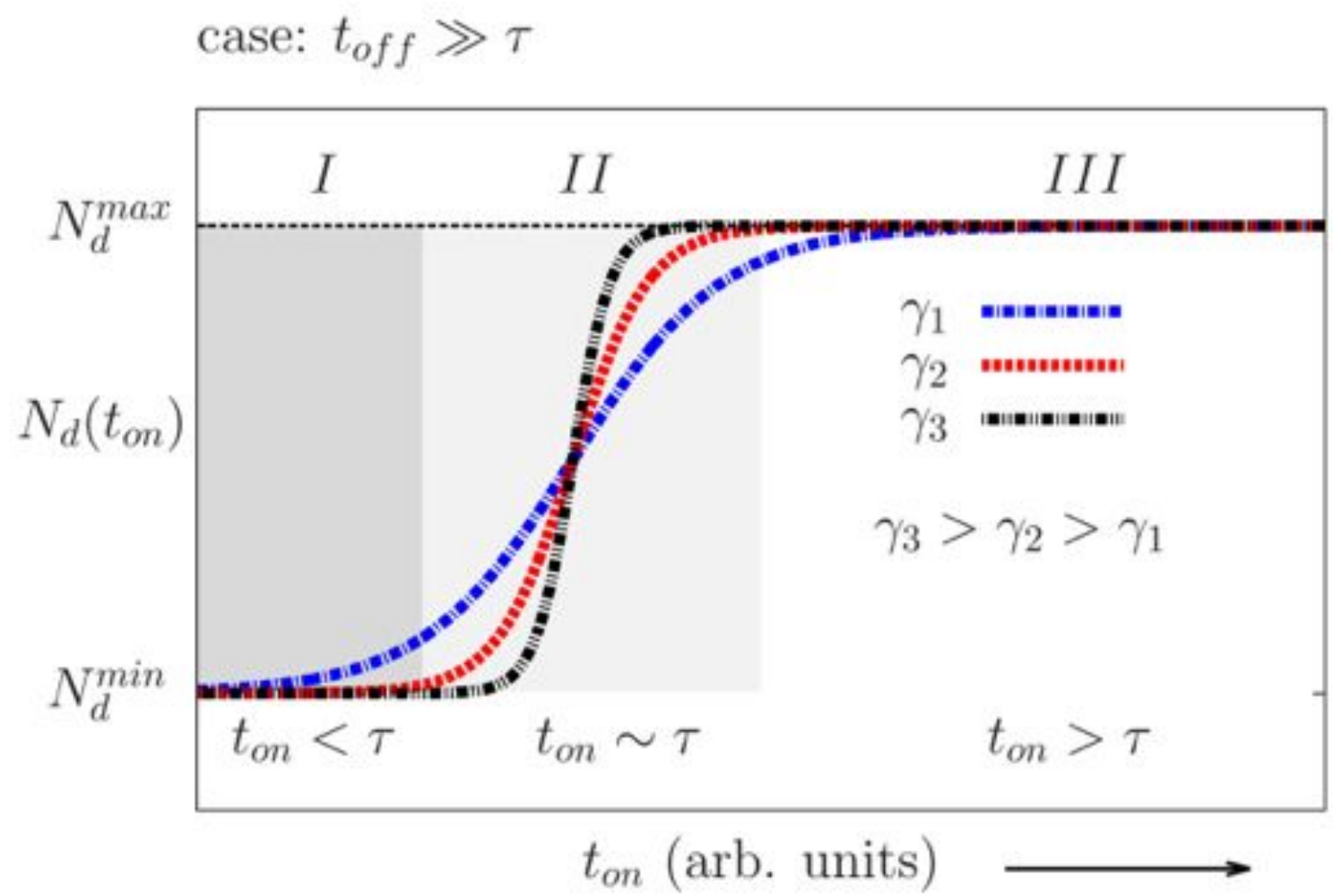

Figure 5.2: Illustration of the number of stable lattice defects, $N_{d}$, after irradiation as a function of increasing $t_{\text {on }}$. Applicable for the case of $\phi_{\text {max }}, t_{\text {off }}$ fixed and $t_{\text {off }} \gg \tau$. The three regimes are denoted as $I, I I$, and $I I I$, illustrating the cases of $t_{\text {on }}<\tau$, $t_{\text {on }} \sim \tau$ and $t_{\text {on }}>\tau$, respectively. Three different damage annealing efficiencies, $\gamma$, are shown to illustrate differences between various ion/energy combinations. 


$$
N_{d}^{\min }=\frac{\Phi_{\text {total }} \alpha}{\phi_{\max }}(1-\gamma),
$$

and shows that in the limit of very low flux, the minimum amount of measured relative disorder, $N_{d}$ can easily be described in terms of two, fundamental material properties, the annealing efficiency $\gamma$, and the linear damage production, $\alpha$.

For region $I I,\left(t_{o n} \sim \tau\right)$ this will be a transient regime that is highly perturbative. For $t_{o n}$ slightly deviating around $\tau$, damage will either be nearly fully anneal, or will build up rapidly. For the latter case, this is due to damage being produced faster than the defect stabilization time. The amount of damage can be predicted, based on Eq. 5.12, given that in this regime, the amount of production and annealing are now both proportional to $\tau$. Given this fact, the relative disorder for this regime can be written as

$$
N_{d}=\frac{\Phi_{\text {total }} \alpha}{\phi_{\max }}\left(\frac{\tau}{t_{\text {on }}}\right)(1-\gamma)
$$

For region $I I I$, this is the saturation regime, when increasing $t_{\text {on }}$ does not result in a measurable increase in stable disorder. One subtle but important point to take away, is that when $t_{o n}<\tau$ (region $I$ ), the amount of damage that can be annealed out is proportional to $t_{o n}$. However, in the case of $t_{o n}>\tau$ (region $I I I$ ), any damage that was created outside of a window of the defect stabilization time is essentially frozen in. Depending on how far $t_{\text {on }}$ has departed from $\tau$ dictates when the saturation regime will occur. If $t_{\text {on }} \gg \tau$, then the maximum relative disorder for the high flux case is given by,

$$
N_{d}^{\max }=\frac{\Phi_{\text {total }} \alpha}{\phi_{\max }} .
$$


In addition to the three regimes seen in Fig 5.2, three curves are also depicted, $\gamma_{1}$, $\gamma_{2}$, and $\gamma_{3}$. These curves represent three different postulated annealing efficiencies, $\gamma$, as defined by Eq. 5.8, which could depend on ion mass, energy, and substrate temperature.

Figure 5.3(a) shows a schematic of the number of stable lattice defects, $N_{d}$, after irradiation as a function of increasing $\Phi_{\text {pulse }}$. Note, the restriction of $t_{\text {off }} \gg \tau$ has been relaxed to $t_{\text {off }}>\tau$. Clearly, in this regime a measurable dependence of $N_{d}$ on $t_{o f f}$ is expected. An increase in $t_{\text {off }}$ should result in a reduction in $N_{d}$. However, as seen in Fig. 5.3(a), the two curves $t_{o f f_{2}}$ and $t_{o f f_{1}}$ should converge to a similar $N_{d}$ at one specific value of $\Phi_{\text {pulse }}$. This unique $\Phi_{\text {pulse }}$ is associated with the flux effect per pulse. In the context of pulsing an ion beam, an interesting concept emerges. Any individual pulse could have a flux effect. That is, each pulse of the beam would have partially damaged regions, and significant diffusion of defects amongst these regions would result in increased disorder. Going from region B (Fig. 5.3(a)) to region A, with decreasing $\Phi_{\text {pulse }}$, partially damaged regions will become less overlapped. As this happens, the convergence in the $N_{d}$ curves will occur when the $\Phi_{\text {pulse }} \leq \frac{1}{L^{2}}$. From this interpretation, an estimation can be placed on the lengths over which defects diffuse beyond the initial ion tracks during the cascade thermalization.

Figure 5.3(b) schematically shows the idea of cascade overlap, borrowed heavily from pioneering work of Crowder and Gibbons [33, 34]. The black regions represent the small amorphous zones, and gray regions represent areas that are partially damaged. Within the gray zones, there will be an outward diffusion of defects (vacancies) that were initially generated in the displacement cascade. In the limit of large fluence per pulse $\left(\Phi_{\text {pulse }}\right)$, the partially damaged regions will be highly overlapped, resulting in higher levels of disorder due to two partially overlapped regions forming a permanently damaged region. This argument is an extension of the fluence effect, whereby 


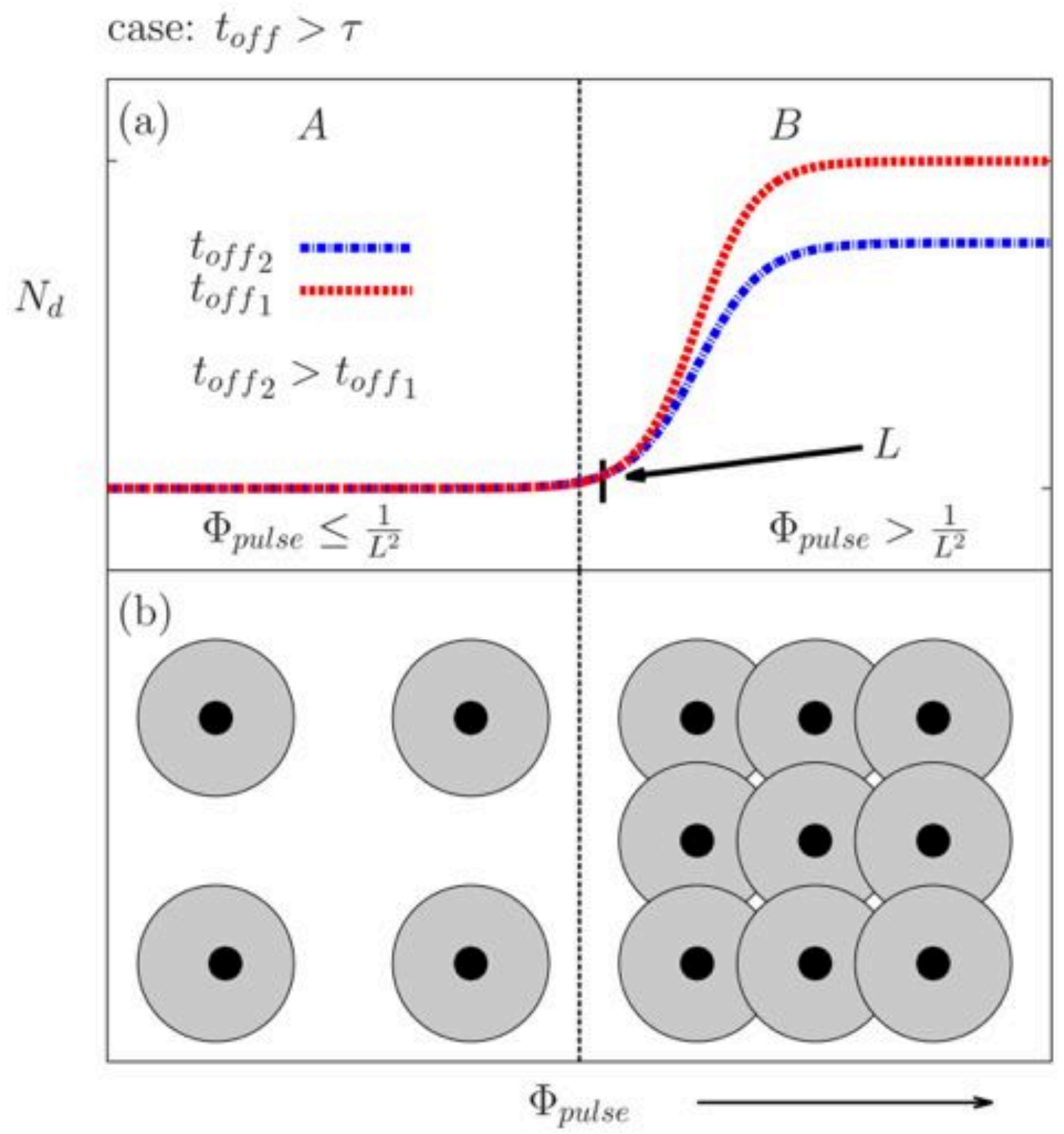

Figure 5.3: (a) Schematic of the number of stable lattice defects, $N_{d}$, after irradiation as a function of increasing $\Phi_{\text {pulse }}$ in the limit of $t_{o f f}>\tau$. Two curves $t_{o f f_{2}}$ and $t_{o f f_{1}}$ show differences in $t_{o f f}$. The distance over which defects leave the initial cascade is denoted as $L$. (b) Pictorially shows cascade overlap. Black regions represent the small amorphous zones, and gray regions represent areas that are partially damaged. 
increasing fluence results in the formation of more successively overlapped regions, thus higher levels of relative disorder. The main difference is, that for the fluence effect, this is postulated to happen over the entire duration of irradiation whereas for pulsed-ion-beam irradiation, this occurs for a given pulse. Between pulses, defect annealing can take place which reduces the overall number of partially damaged regions (or their size).

The discussion thus far has assumed athermal conditions during irradiation, where defect interactions take place at fixed temperature. This is not an unrealistic assumption for irradiation conditions where the temperature is fixed, $t_{\text {off }}$ is long, and minimal beam heating can occur. For practical situations, probing the defect lifetime requires maximizing $\phi_{\max }$, and minimizing $t_{o n}$, all the while maintaining $\Phi_{\text {pulse }} \leq \frac{1}{L^{2}}$. This will ensure minimal flux effect per pulse for short $t_{o n}$ and will allow probing fundamentally smaller timescales. However, as $t_{o n}$ is increased, (or $t_{\text {off }}$ decreased), the possibility of beam heating is very likely. A small correction is proposed to account for this, which is to assume an Arrenhius relationship for $\tau$, given by,

$$
\tau=\tau_{0} e^{-\frac{E_{b}}{k T}},
$$

where $\tau$ is the defect stabilization time, $\tau_{0}$ is the defect stabilization time in the limit of low temperature, $E_{b}$ is the defect activation energy, $k$ is Boltzmann constant, and $T$ is the substrate temperature. For situations involving large $\Phi_{\text {pulse }}$ and very short $t_{\text {off }}$ it may prove necessary to account for the effect of temperature on the measurement of the defect stabilization time. 
Rev. 1.2

\subsection{Experimental setup}

Figure 5.4 shows the pulsed-ion-beam setup that was used in this dissertation. The pulsed beam component was connected to the $4 \mathrm{MV}$ ion accelerator [National Electrostatics Corporation (NEC), model 4UH] at Lawrence Livermore National Laboratory. The modified portion of the beam line required the removal of a traditional NEC raster scanner and replacing it with a pulsed-ion-beam setup depicted in Fig. 5.4.

A series of electrostatic plates perform beam deflection, thus, allowing precise pulsing of an ion-beam on and off the target. Two apertures were used with the first being used to define the beam and the second to remove the divergent portion of the beam. This method ensures that a parallel, highly collimated beam is delivered on target.

The area of the aperture is known from precise measurement and allows for an accurate determination of the total accumulated fluence from the integrated charge. Located between the two apertures is a suppression ring that is biased to produce a field which will cause the path of secondary electrons to be deflected. Secondary electrons are produced due to collisions with the metal apertures. Finally, the pulsed beam is delivered on target with the charge from the incident ions being collected from the actual sample surface. More details are given in Fig. 5.5 and 5.6 pertaining to the electrostatic deflection system and sample holder in the target chamber. The combination of each of these components comprise the charge integration system. Table 5.1 provides a concise description of the bias voltages and the purpose of each component described in Figs. 5.4, 5.5 and 5.6. 

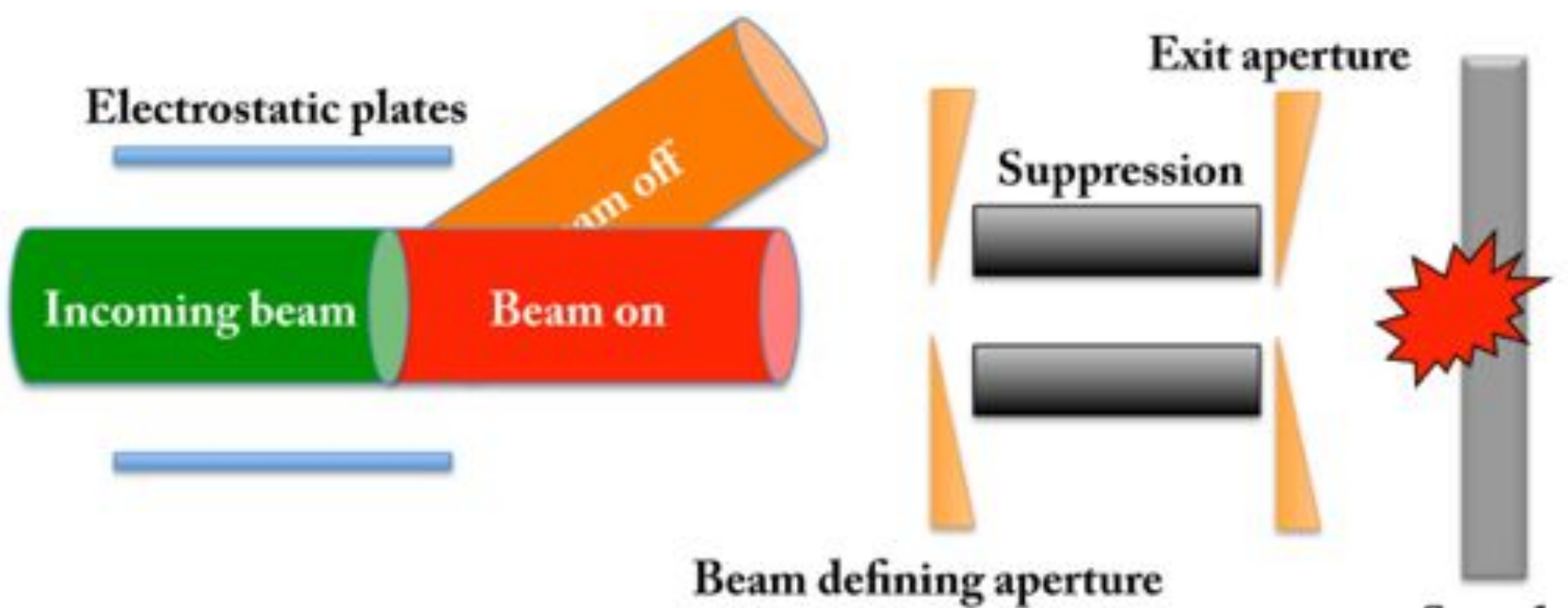

Beam defining aperture

Sample

Figure 5.4: A diagram of the major components in the pulsed ion irradiation facility which was constructed and operated at Lawrence Livermore National Laboratory for the experiments performed in this section. For clarity, only one set of electrostatic deflection plates is shown. In the actual setup, there were four plates as shown in Fig 5.5. Charge collection was performed using the actual sample surface which formed a portion of the Faraday cup arrangement. The suppression ring was biased to 180 volts to eliminate the role of secondary electrons in the charge integration. Actual sample holder setup is shown in Fig 5.6. 
Figure 5.5 is a schematic of the connections to the pulsed ion beam parallel plates used for beam deflection. One plate, which is traditionally connected to the NEC raster-scanner system, was instead connected to a Trek high voltage power supply that is pulsed using a Stanford Research pulse generator. This plate performed the actual pulsing and is labeled as "Pulse" in Fig. 5.5. The second plate of vertical pair of electrostatic plates, labeled as "Y-Wiggle" in Fig. 5.5, was used to provide a small sinusoidal ripple. This plate was driven by a waveform generator, oscillating at $\sim 7$ $\mathrm{Hz}$, which was connected to a second Trek high voltage power supply (operating in bi-polar mode).

Of the two remaining plates, only one is used and the purpose is to perform a slight beam wiggle in the $x$-direction. This plate is labeled as "X-Wiggle" in Fig. 5.5. This plate is driven by a second waveform generator, oscillating at $\sim 1 \mathrm{~Hz}$, which was connected to a Stanford Research high voltage power supply (operating in unipolar mode). Table 5.2 provides a concise description of the bias voltages, voltage operating range, the frequency of operation and the type of waveform used on each plate. It should be noted that the parameters that are described in this study are used to steer a beam of $500 \mathrm{keV}$ Ar ions. Due to the nature of deflecting a beam of charged particles, the amount of field (i.e. voltage) required will be independent of the ion mass, however, it will vary as one over the square root of the energy. Therefore, in order to pulse a beam of higher energy particles, the voltages will need to be increased accordingly.

The pulsing is achieved by varying the frequency and delay on a Stanford Research pulse generator. This is easily accomplished knowing the relationship between the frequency and active and passive portions of a duty cycle. The duty cycle is defined 
Rev. 1.2

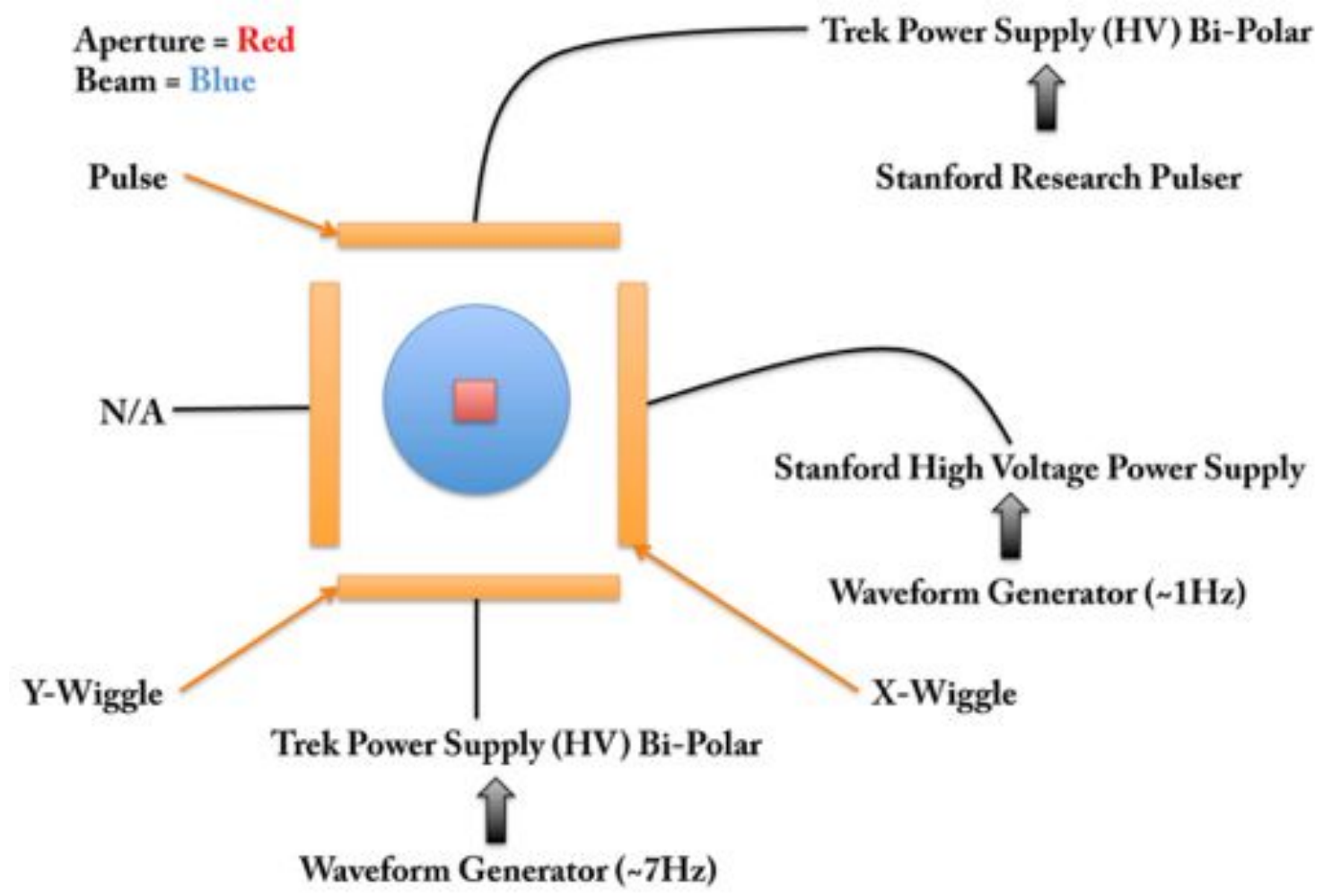

Figure 5.5: A diagram of the connections to the parallel plates (shown in orange) which performed beam pulsing and wiggling. The aperture across which the ion beam was pulsed is shown in red with the ion beam shown as blue. In addition to the first aperture, there is a second exit aperture, shown in Fig 5.4 which would be directly downstream and therefore not visible in this schematic. 
Rev. 1.2

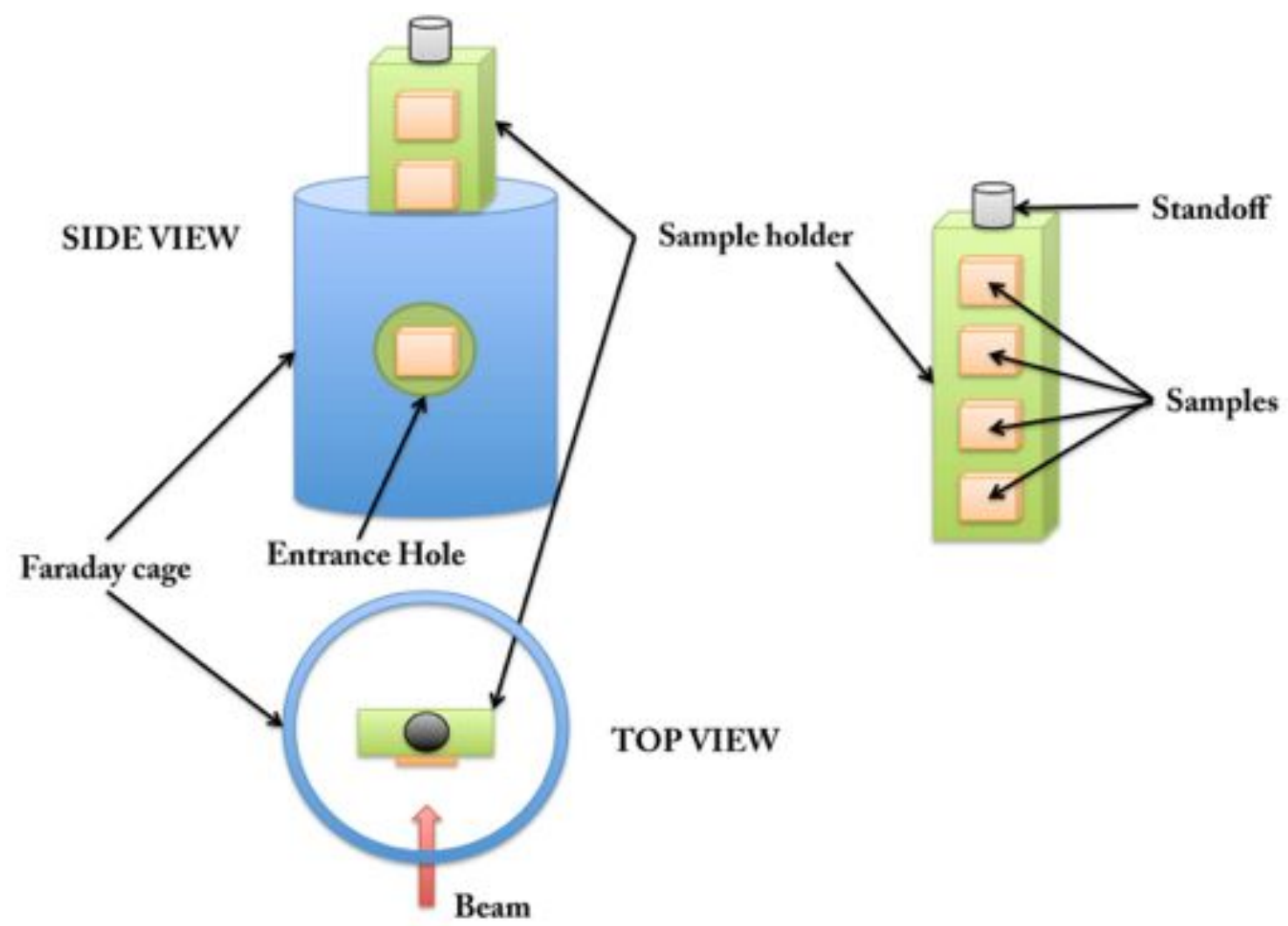

Figure 5.6: A schematic drawing (both top and side view) of the sample holder and Faraday cage used in the pulsed beam setup. 
as,

$$
C_{d}=\frac{t_{o n}}{t_{o n}+t_{o f f}}
$$

where $\mathrm{C}_{d}$ is the duty cycle and $\mathrm{t}_{o n}$, $\mathrm{t}_{\text {off }}$ represent the active and passive portion of the beam, respectively. The average flux (actually measured on a meter) should then be equal to the maximum instantaneous flux multiplied by the duty cycle.

Given fixed values for both $t_{o n}$ and $t_{\text {off }}$, the frequency required to achieve those values can be calculated as,

$$
\Gamma=\left(t_{o n}+t_{o f f}\right)^{-1}
$$

where $\Gamma$ is the frequency and $\mathrm{t}_{o n}, \mathrm{t}_{\text {off }}$ are, again, the active and passive portion of the beam, respectively.

Figure 5.6 shows the sample holder which was machined specifically for pulsed beam irradiation, the Faraday cage constructed of wire mesh, and the entrance hole in the Faraday cage through which the beam entered. The Faraday cage has two purposes. First, ions striking the sample surface will generate secondary electrons which must be captured back on the sample holder for complete charge collection. Second, some amount of scattered beam, whether it occurs in the beam leg directly before the apertures or after striking the aperture will collide with the walls of the chamber and other metal components in the target chamber also generating secondary electrons which must not be collected on the sample holder. These two processes pose a significant challenge to charge collection and therefore a isolated, grounded Faraday cage acts to shield the sample holder.

The sample holder was isolated from the target chamber using a ceramic standoff and positively biased (see Table 5.1) to capture ejected secondary electrons. Samples were attached to the sample holder using either carbon tape or copper powder impregnated vacuum grease. The top of the chamber was designed to allow an at- 
Table 5.1: A summary of the components, their bias voltage, and their purpose in the pulsed-ion-irradiation setup used to collect charge (i.e. calculate the fluence delivered) as shown in Figs. 5.4, 5.5 and 5.6.

\begin{tabular}{|l|c|c|}
\hline Component & Bias & Purpose of the Component \\
\hline \hline Entrance aperture & Ground $(0 \mathrm{~V})$ & Reduce beam size \\
Suppression ring & $-700 \mathrm{~V}$ & Steer off secondary electrons \\
Exit aperture & Ground $(0 \mathrm{~V})$ & Ensure parallel beam \\
Faraday cage & Ground $(0 \mathrm{~V})$ & Reduce secondary current on the holder \\
Sample holder & $+180 \mathrm{~V}$ & Collect charge/collect all secondaries \\
\hline
\end{tabular}

Table 5.2: A description of the plates (shown in Fig. 5.5), their bias voltage, voltage range, frequency, and the type of waveform used for the pulsed-ion-irradiation setup shown in Fig. 5.4.

\begin{tabular}{|l|c|c|c|c|}
\hline Plate & Bias $(\mathrm{V})$ & Range $(\mathrm{V})$ & Frequency $(\mathrm{Hz})$ & Waveform \\
\hline \hline X-wiggle & 0 & $(-80$ to $+80 \mathrm{~V})$ Bipolar & 7.897 & Square \\
Y-wiggle & +360 & $(0$ to $+80 \mathrm{~V})$ Unipolar & 1.7 & Sine \\
Pulse & 0 & +400 or 0 & Variable & Pulse \\
\hline
\end{tabular}

tachment to the sample holder which can be swept through $360^{\circ}$ thus permitting a wide range of irradiation conditions. In addition, the target chamber top was designed to allow vertical movement of the sample holder, therefore multiple samples are placed on the holder and in turn, each one is brought into the beam for pulsed irradiation.

5.4 Method to measure the time constant of dynamic annealing in silicon at room temperature $^{\nmid}$

In this section, we focus on the time scale of DA processes; i.e., a characteristic time constant $\tau$ over which the dominant processes of defect evolution persist after the thermalization of collision cascades. Such a time constant $\tau$ is determined by the thermal stability, effective diffusivity, and specific interaction processes of radiation-

$\nmid$ This work was reprinted from Physical Review Letters, Vol 109 / Issue 9, M. T. Myers, S. Charnvanichborikarn, L. Shao, and S. O. Kucheyev, "Pulsed ion beam measurement of the time constant of dynamic annealing in Si", Pages No. 095502-1 - 095502-4, Copyright (2012), with permission from American Physical Society. 
generated defects. Knowledge of $\tau$ is important for the development of physically sound models of damage accumulation in solids in order to control and fully exploit the effects of radiation damage. In particular, it is critical for extending laboratory findings to nuclear material lifetimes and dynamic regimes as well as to the time scales of geological storage of nuclear waste [15, 27, 31].

Values of $\tau$ are, however, largely unknown even for arguably the best studied material system - single crystalline $\mathrm{Si}$ at RT. Indeed, current estimates of $\tau$ for $\mathrm{Si}$ at RT range from $\sim 10^{-10}$ to $\gtrsim 10^{2} \mathrm{~s}[39,135,139-142]$, inconsistency of 12 orders of magnitude! Such a large scatter in the estimates of $\tau$ is related to the fact that calculations and measurements of $\tau$ are not straightforward. Indeed, although molecular dynamics (MD) simulations are currently practical for following defect evolution only for $\lesssim 10^{-8} \mathrm{~s}$, a number of MD studies by different groups [140-142] have suggested that defect evolution processes in $\mathrm{Si}$ at RT essentially cease for times $\gtrsim 10^{-10}-10^{-9} \mathrm{~s}$ after cascade generation.

Other estimates of $\tau$ have involved an analysis of the flux effect (also often referred to as the flux effect); i.e., the dependence of ion-beam-produced stable lattice disorder on the flux when all the other experimental parameters are kept constant $[39,43,135$, 136]. A flux effect is observed when defect stabilization time $\tau$ is comparable to the average time interval between the formation of spatially overlapping damage zones originating from different collision cascades. Lateral dimensions of such damage zones are determined by both the average size of ballistic collision cascades and effective defect diffusion lengths, $L_{d}$. Hence, the flux effect combines both temporal $(\tau)$ and spatial $\left(L_{d}\right)$ information that must be separated in order to obtain $\tau$. Such estimates of $L_{d}$ (and, hence, the extraction of $\tau$ from the experimental flux dependence of disorder) require serious assumptions about the explicit defect interaction processes $[39,135,136]$. 
Spatial and temporal information can be separated in experiments with pulsed ion beams. Such a method was used by Linnros and co-workers [129, 130], who measured effective time constants of $\gtrsim 1 \mathrm{~s}$ for the process of ion-beam-induced epitaxial recrystallization of $\mathrm{Si}$ at elevated temperatures $\left(200-300{ }^{\circ} \mathrm{C}\right)$. In this section, we use a similar pulsed beam approach and find a characteristic DA time constant of $\sim 6 \mathrm{~ms}$ in Si bombarded at RT with $500 \mathrm{keV}$ Ar ions.

Float-zone grown (100) Si single crystals (with a resistivity of about $5 \Omega \mathrm{cm}$ ) were bombarded at RT with $500 \mathrm{keV}^{40} \mathrm{Ar}^{+}$ions at $7^{\circ}$ off the [100] direction. Ion irradiation and ion beam analysis were carried out with the $4 \mathrm{MV}$ ion accelerator (National Electrostatics Corporation, model 4UH) at Lawrence Livermore National Laboratory. To improve thermal contact, samples were clamped to an Al holder with a thin layer of Cu-powder-impregnated thermal grease in between. To avoid complexity related to differences between instantaneous and average fluxs inherent to experiments with rastered ion beams, all irradiations were performed in a broad beam mode $[43,143]$. The central portion of the beam, estimated to be wider than $\sim 15 \mathrm{~mm}$ in diameter, was selected with a $4 \times 5 \mathrm{~mm}^{2}$ final beam defining aperture. A small sine ripple $(\sim 7 \mathrm{~Hz})$ was applied to a set of electrostatic deflection plates in the horizontal direction to improve beam uniformity. This ripple extended the beam width by only $\sim 10 \%$ compared to the unperturbed beam. Beam pulsing was performed by applying high voltage pulses to a pair of plates deflecting the beam in the vertical direction off the final beam defining aperture.

After Ar ion irradiation, lattice disorder was measured by Rutherford backscattering/channeling (RBS/C) spectrometry with $2 \mathrm{MeV}^{4} \mathrm{He}^{+}$ions incident along the [100] direction and backscattered into a detector at $164^{\circ}$ relative to the incident beam direction. All RBS/C spectra were analyzed with one of the conventional algorithms [70] for extracting the effective number of scattering centers (referred to below as 
Rev. 1.2

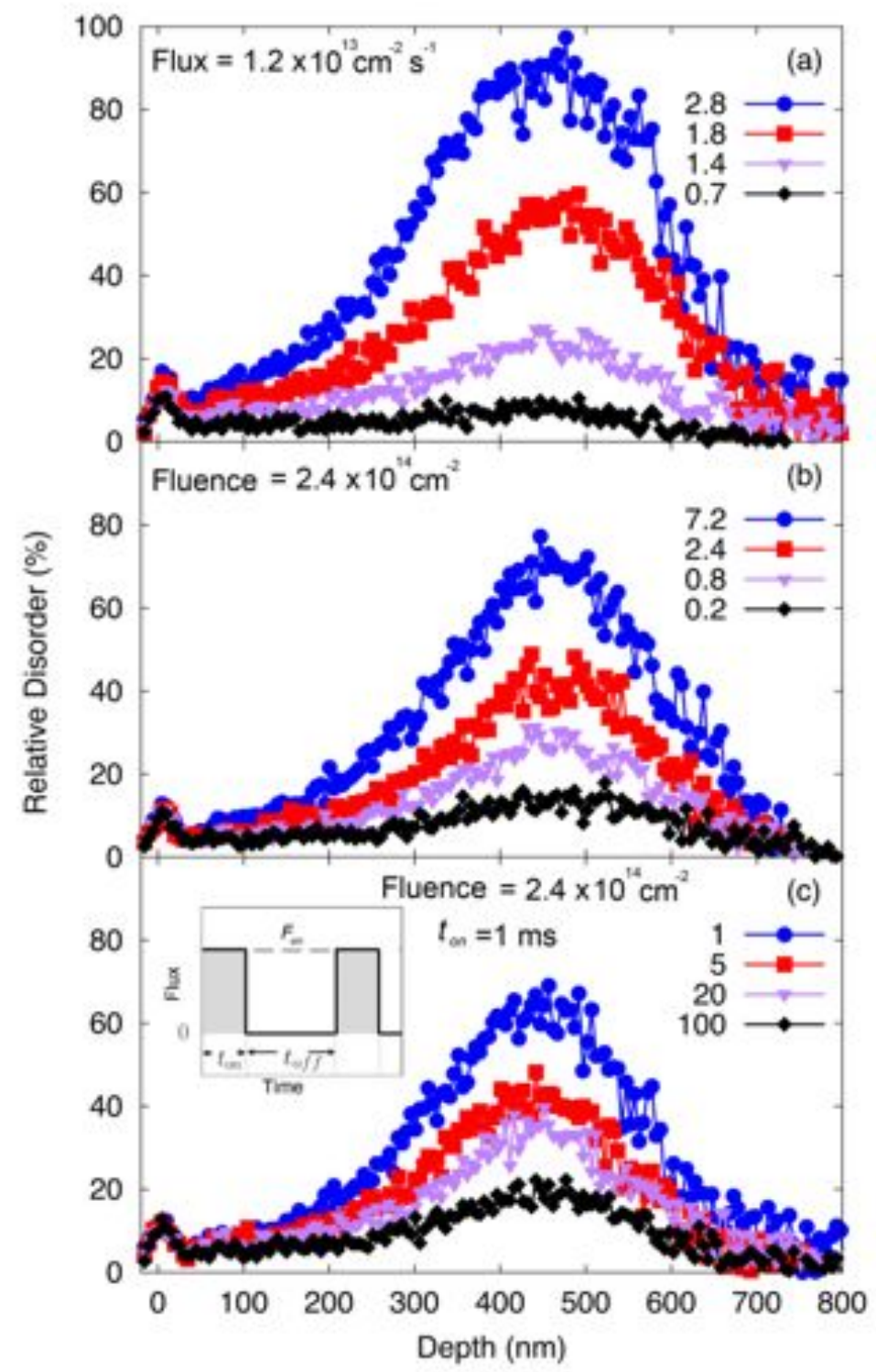

Figure 5.7: Selected depth profiles of relative disorder in Si bombarded at RT by 500 $\mathrm{keV} \mathrm{Ar}$ ions (a) with a continuous beam with a constant flux of $1.2 \times 10^{13} \mathrm{~cm}^{-2} \mathrm{~s}^{-1}$ to various fluence (given in the legend in units of $10^{14} \mathrm{~cm}^{-2}$ ), (b) with a continuous beam to the same fluence of $2.4 \times 10^{14} \mathrm{~cm}^{-2}$ with different fluxs (given in the legend in units of $10^{12} \mathrm{~cm}^{-2} \mathrm{~s}^{-1}$ ), and (c) with a pulsed beam with different values of $t_{\text {off }}$ (given in the legend in units of $10^{-3} \mathrm{~s}$ ) and all the other parameters fixed (fluence $=$ $2.4 \times 10^{14} \mathrm{~cm}^{-2}, t_{o n}=1 \mathrm{~ms}$, and $F_{o n}=1.2 \times 10^{13} \mathrm{~cm}^{-2} \mathrm{~s}^{-1}$ ). The inset in (c) shows a schematic of the time dependence of the flux for pulsed beam irradiation, defining $t_{o n}, t_{o f f}$, and $F_{o n}$. 
"relative disorder").

Three sets of interrelated experiments were performed. First, we studied the damage buildup with a continuous beam with a constant flux and varied fluence in the range of $(0.7-5.0) \times 10^{14} \mathrm{~cm}^{-2}$. Based on the buildup curve measured, for further experiments, we selected a fluence of $2.4 \times 10^{14} \mathrm{~cm}^{-2}$ in a non-linear damage buildup regime where DA processes are particularly pronounced [43]. A second set of experiments involved bombardment with a continuous beam to a fluence of $2.4 \times 10^{14}$ $\mathrm{cm}^{-2}$ with different fluxs. Third, we measured a dependence of lattice damage on the duration of the passive part of the ion beam cycle with all the other irradiation parameters kept constant.

Figure 5.7(a) shows selected depth profiles of lattice disorder for continuous beam irradiation to different fluence with a constant flux of $1.2 \times 10^{13} \mathrm{~cm}^{-2} \mathrm{~s}^{-1}$. These depth profiles are bimodal, with the first peak reflecting the damage nucleated at or near the sample surface and the second broad bulk peak centered on a depth of $\sim 450 \mathrm{~nm}$, where the nuclear energy loss profile is maximum [20]. The bulk damage buildup with increasing ion fluence, better illustrated in Fig. 5.8, is consistent with a number of previous systematic studies [36, 43, 144]. Disorder increases monotonically until full lattice amorphization is achieved. For fluence of $\lesssim 1.4 \times 10^{14} \mathrm{~cm}^{-2}$, damage accumulates close-to-linearly with fluence. For larger fluence, a super-linear increase in disorder is seen in Fig. 5.8. Such super-linearity has been attributed to critical energy density effects [145]. Fluence rate (i.e. flux) effect studies of Titov and Carter [43] have shown that DA processes in Si are particularly pronounced in such a nonlinear regime. Hence, for the DA studies discussed below, we have selected a fluence of $2.4 \times 10^{14} \mathrm{~cm}^{-2}$ (marked by a star in Fig. 5.8).

Figure 5.7(b) shows selected depth profiles of disorder in Si irradiated with a continuous beam to a fluence of $2.4 \times 10^{14} \mathrm{~cm}^{-2}$ with different fluxs [in the range of 
$\left.(0.1-10) \times 10^{12} \mathrm{~cm}^{-2} \mathrm{~s}^{-1}\right]$. It is seen from Fig. 5.7(b) that lower fluxs result in less stable damage in the bulk peak region but a negligible effect on damage accumulation within $\sim 40 \mathrm{~nm}$ from the sample surface. This observation is consistent with several previous reports $[42,43,138]$. It suggests different physical mechanisms of bulk and surface disordering.

Figure 5.7(c) shows depth profiles of disorder in Si irradiated with a pulsed beam when the total fluence was split into a series of equal pulses. The inset in Fig. 5.7(c) shows a time dependence of the flux on the target and defines pulsed-beam-related parameters $t_{o n}, t_{o f f}$, and $F_{o n}$. For different curves shown in Fig. 5.7(c), all the irradiation parameters were kept constant (a fluence of $2.4 \times 10^{14} \mathrm{~cm}^{-2}, t_{\text {on }}=1 \mathrm{~ms}$, and $F_{o n}=1.2 \times 10^{13} \mathrm{~cm}^{-2} \mathrm{~s}^{-1}$ ) except for $t_{\text {off }}$, the duration of the passive part of the ion beam cycle. Figure 5.7(c) reveals that the amount of stable disorder in the bulk decreases with increasing $t_{o f f}$, while surface damage is essentially independent of $t_{o f f}$.

Interestingly, for both pulsed beam irradiation [Fig. 5.7(c)] and continuous beam irradiation with different fluxs [Fig. 5.7(b)], defect dynamics effects are evidenced only for the bulk and not the surface peak of disorder. This suggests that the same DA mechanisms are responsible for fluence-rate and pulsed-beam effects. The similarity between pulsed irradiation and variable flux irradiation is further supported by Fig. 5.9, which compares the dependence of stable damage on the average flux $\left[F_{\text {avg }}=F_{o n} /\left(1+t_{o f f} / t_{o n}\right)\right]$ for cases of pulsed and continuous beam irradiation. ${ }^{1}$ Figure 5.9 shows that, for a given average flux, pulsed and continuous beam irradiation regimes create similar (although not identical) levels of stable disorder, supporting the above suggestion that the same DA processes are responsible for fluence-rate and

\footnotetext{
${ }^{1}$ Note that the average dose rate is not conserved in experiments with variable $t_{\text {off }}$ and constant $t_{\text {on }}$ and $F_{\text {on }}$.
} 


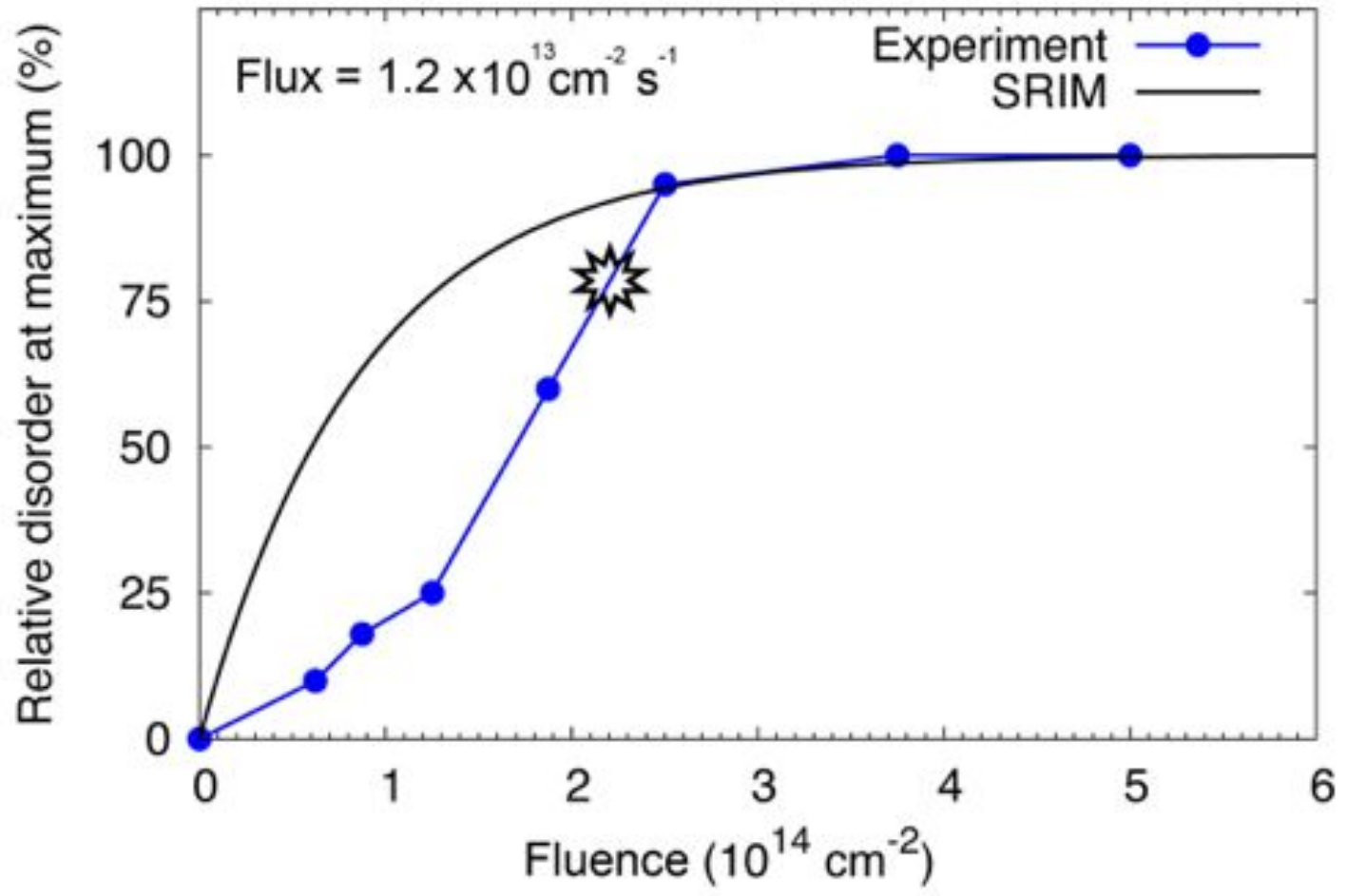

Figure 5.8: Fluence dependence of relative disorder at the maximum of the bulk defect peak for Si bombarded at room temperature by $500 \mathrm{keV}$ Ar ions with a flux of $1.2 \times 10^{13} \mathrm{~cm}^{-2} \mathrm{~s}^{-1}$ [based on ion channeling data such as shown in Fig. 5.7(a)]. The dash line shows a SRIM-code-predicted [20] dependence, taking into account damage saturation upon amorphization. The star denotes the fluence used in studies of defect dynamics. 


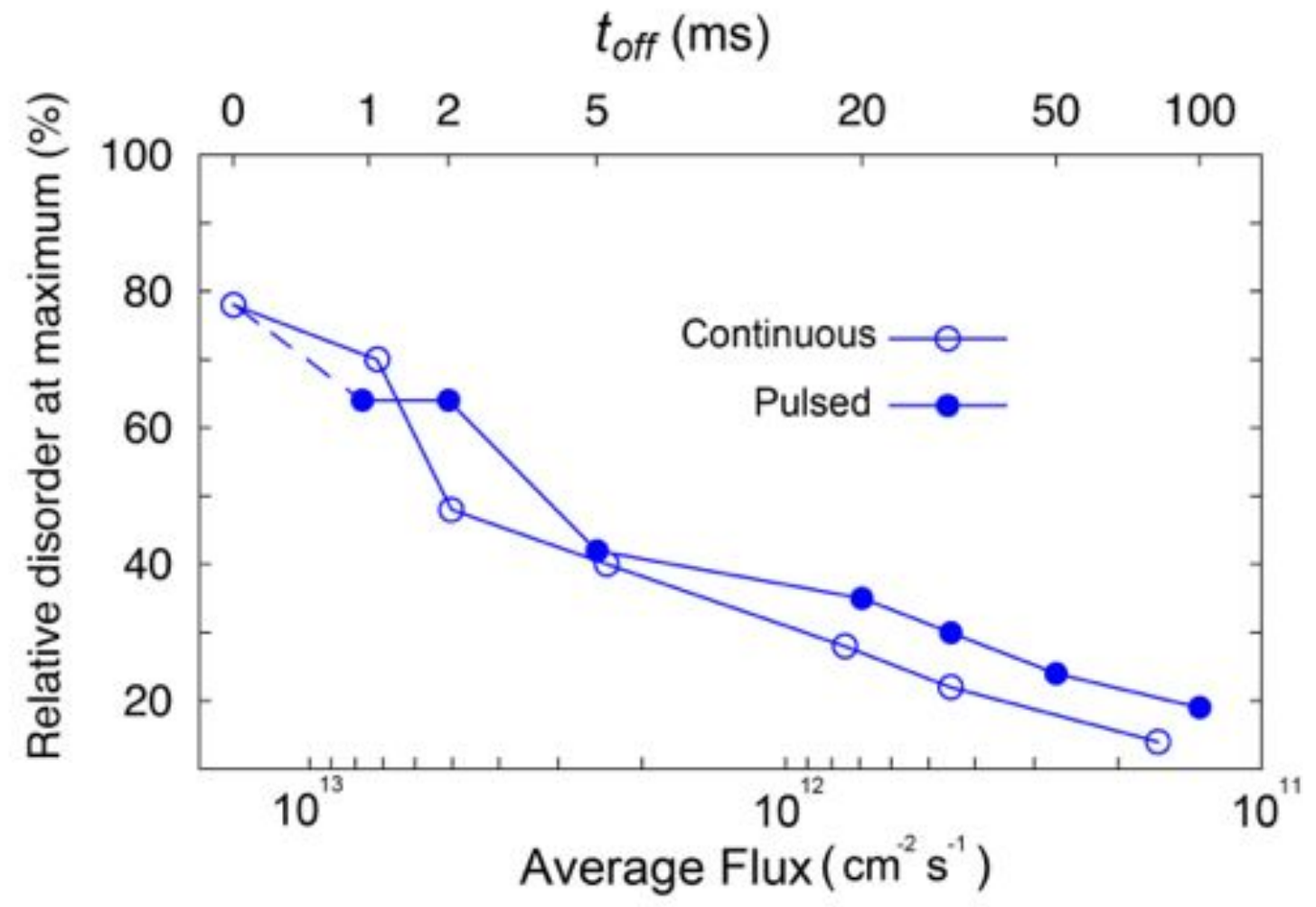

Figure 5.9: Dependence of relative disorder at the maximum of the bulk defect peak on the average flux, $F_{\text {avg }}$ (for both continuous and pulsed beam irradiation) [bottom axis] and on $t_{\text {off }}$ (for pulsed beam irradiation) [top axis] for Si bombarded at room temperature by $500 \mathrm{keV}$ Ar ions to a fluence of $2.4 \times 10^{14} \mathrm{~cm}^{-2}$. For pulsed experiments, the maximum flux was $1.2 \times 10^{13} \mathrm{~cm}^{-2} \mathrm{~s}^{-1}$ and $t_{\text {on }}$ [defined in the inset of Fig. 5.7(c)] was $1 \mathrm{~ms}$. 
pulsed-beam effects.

As mentioned above, in contrast to the case of flux data, the DA time constant $\tau$ is clearly revealed in pulsed beam experiments. Figure 5.10 shows a trend of reduced bulk disorder with increasing $t_{\text {off }}$. An increase in $t_{\text {off }}$ from $0 \mathrm{~ms}$ (i.e., a continuous beam) to $50 \mathrm{~ms}$ results in an $\sim 72 \%$ decrease in bulk disorder. For $t_{\text {off }} \gtrsim 50 \mathrm{~ms}$, the disorder profile is essentially independent of $t_{\text {off }}$ (within experimental errors), indicating that $\tau$ is on the order of magnitude of $10 \mathrm{~ms}$.

The dependence of damage on $t_{\text {off }}$ is related to the interaction of defects generated not only in different collision cascades but also by different pulses. As the beam is pulsed off the target, the defect concentration decreases via DA. For irradiation with $t_{\text {off }}>>\tau$, DA processes have essentially decayed in time intervals between individual ion pulses. This behavior can be treated phenomenologically in terms of competitive damage generation and annealing processes, as has been done by Carter [146] for a semi-quantitative description of the flux effect. Figure 5.10 suggests that defect evolution follows a second order behavior. Indeed, the dependence of the maximum defect concentration $\left(n_{\text {def }}\right)$ obeys a second order kinetic equation $\left(\frac{\partial}{\partial t} n_{d e f} \propto n_{d e f}^{2}\right)$ :

$$
n_{d e f}\left(t_{o f f}\right)=n_{\infty}+\frac{n_{0}-n_{\infty}}{1+\frac{t_{o f f}}{\tau}}
$$

where $n_{0}$ and $n_{\infty}$ are defect concentrations for $t_{\text {off }}=0$ and $\infty$, respectively. A fit to the data in Fig. 5.10 with a nonlinear least-squares Marquardt-Levenberg algorithm yields $n_{0}=78$ at. $\%, n_{\infty}=17$ at. $\%$, and $\tau=6 \pm 1 \mathrm{~ms}$. Attempts to fit the data shown in Fig. 5.10 with a single exponential decay curve (i.e., the solution of the first order kinetic equation) have resulted in an inferior fit compared to the case of fitting with the solution of the second order kinetic equation discussed in the text. However, even such a single exponential decay fit, albeit poor, yields $\tau=7 \pm 2 \mathrm{~ms}$, 
demonstrating that the value of $\tau$ measured with the pulsed beam method is weakly dependent on the particular model of defect interaction processes assumed.

The DA efficiency, which we define here as $\frac{n_{0}-n_{\infty}}{n_{0}}$, is $\sim 78 \%$. Not surprisingly, a $\tau$ of $6 \mathrm{~ms}$ is in the range of previous estimates $\left(10^{-10}-10^{2} \mathrm{~s}\right)[39,135,139-142]$. It is, however, two orders of magnitude smaller than the characteristic time constant of the ion-beam-induced recrystallization process in $\mathrm{Si}$ at elevated temperatures studied by Linnros and co-workers $[129,130]$. This is consistent with an expectation that $\tau$ depends both on material properties and irradiation conditions (i.e., substrate temperature; ion fluence; the maximum flux; and the average density of collision cascades [147], determined by ion mass and energy). Future pulsed beam irradiation experiments should gain insight into how the DA time constant $\tau$ in Si depends on the type and concentration of dopants and on irradiation parameters. Data obtained with this method could also have important implications for the development of physically sound models of damage accumulation in solids. Clearly, a successful model for $\mathrm{Si}$

should include specific defect interaction processes with characteristic relaxation times and kinetics revealed by this work.

This pulsed-beam method could also be applied to study defect dynamics in technologically relevant materials other than Si. Of particular interest are material systems exposed to neutron irradiation. In such cases, understanding fundamental timescales of post-cascade-thermalization processes is crucial due to an inherent problem in the emulation of neutron and radioactive-decay-induced damage by ion irradiation, related to a large difference in rates of displacement generation between reactor operation or spent nuclear fuel storage conditions and ion irradiation experiments in the laboratory.

This section could be summarized as follows: (i) we have demonstrated an ex- 
Rev. 1.2

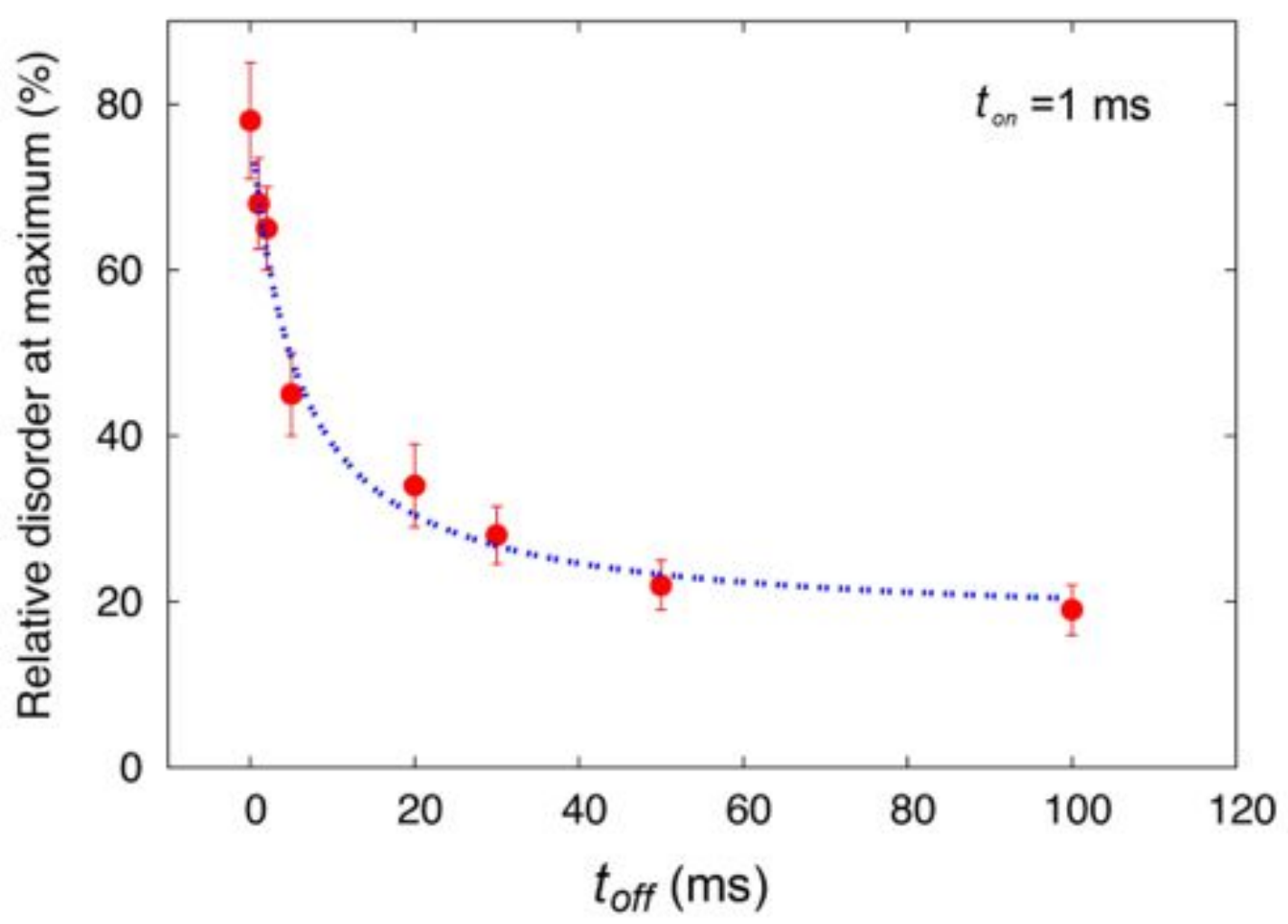

Figure 5.10: Dependence of relative disorder at the maximum of the bulk defect peak on the passive portion of the beam $t_{\text {off }}$, with a fitting curve of the second order rate equation, discussed in the text, shown by a dash line. Error bars correspond to peak-to-peak noise in RBS/C-derived disorder profiles such as shown in Fig. 5.7(c). 
perimental pulsed ion beam method to measure characteristic time constants of DA processes in solids under irradiation, (ii) the $\mathrm{DA}$ time constant in $\mathrm{Si}$ at $\mathrm{RT}$ is $\sim 6 \mathrm{~ms}$, (iii) the defect relaxation behavior measured for $\mathrm{Si}$ at RT suggests a second order kinetic process, and (iv) these results should stimulate future studies of dynamic defect interaction processes in other technologically relevant materials.

5.5 Method to measure the diffusion length of dynamic annealing in silicon at room temperature

Radiation-generated point defects in solids often experience DA — diffusion and interaction processes after the thermalization of collision cascades. The length-scale of DA could be described by the characteristic defect diffusion length $\left(L_{\mathrm{d}}\right)$. In this section, we propose to measure the $L_{\mathrm{d}}$ by a pulsed-beam method. Our approach is based on the observation of enhanced defect production when, for individual ion pulses, the average separation between adjacent damage regions is smaller than the $L_{\mathrm{d}}$. We obtain a $L_{\mathrm{d}}$ of $\sim 30 \mathrm{~nm}$ for float-zone Si crystals bombarded at RT with 500 $\mathrm{keV}$ Ar ions.

Despite many decades of extensive research, understanding response of solids to irradiation with energetic particles remains a major materials physics challenge $[22,24,25]$. This is directly related to the mesoscale nature and complexity of radiation damage phenomena. Collisional processes by which the incident particle slows down result in the formation of vacancies and interstitials and the development of collision cascades. This ballistic stage of defect production is considered to be well understood (excluding cases when cascades are non-linear) [22, 24, 25]. After cascade thermalization, point defects can experience migration and interaction, commonly referred to as DA processes. It is the DA that, in most practical cases, largely determines the form and extent of stable lattice damage in solids after irradiation 
and materials' "radiation-resistance" $[22,24,25]$. Such DA processes are complex and remain poorly understood. They depend non-trivially on both the material itself and irradiation conditions that include energy, mass, fluence, and flux of bombarding particles as well as target temperature [22, 24, 25].

The length- and time-scales of DA could be described by characteristic diffusion lengths $\left(L_{\mathrm{d}}\right)$ and lifetimes $(\tau)$ of point defects. The $L_{\mathrm{d}}$, which is the focus of the present study, describes the average distance over which defects diffuse outward from the ballistic cascade before they annihilate or get trapped at other lattice defects during the time $\tau$ after cascade thermalization. However, this basic parameter, $L_{\mathrm{d}}$, is still not well known even for arguably the best studied material system like singlecrystalline $\mathrm{Si}$. Indeed, for $\mathrm{Si}$ at $\mathrm{RT}$, a wide range of $L_{\mathrm{d}}$ values has been reported, from $\sim 10$ to $2000 \mathrm{~nm}[39,44,138,139,148-154]$.

This situation is related to difficulties with direct measurements of the $L_{\mathrm{d}}$ and, possibly, to its expected dependence on irradiation conditions and the quality of the starting material. Many DA studies have traditionally involved measurements of the dependence of damage production on the flux when all the other experimental parameters are kept constant $[22,24,25,39,42-44,138]$. A flux effect is observed when $\tau$ is comparable to the average time interval between the formation of damage zones, originating from different collision cascades, at distances $\lesssim L_{\mathrm{d}}$. Hence, the difficulty of the flux effect approach is related to the fact that the spatial $\left(L_{\mathrm{d}}\right)$ and temporal $(\tau)$ contributions are convoluted, and their separation requires making assumptions about explicit defect interaction processes [39, 137, 138].

The $L_{\mathrm{d}}$ can also be estimated from measurements of dopant diffusion profiles [148, 155-157]. This, however, requires making additional assumptions about diffusion mechanisms and has further complications that such diffusion experiments are performed at elevated temperatures (for example, $\gtrsim 600{ }^{\circ} \mathrm{C}$ for $\mathrm{Si}$ ), and, hence, their 
Rev. 1.2

results need to be extrapolated to lower irradiation temperatures that are often of practical interest.

Other approaches to estimating $L_{\mathrm{d}}$ 's include transmission electron microscopy $[139,154]$ and studies of Si bombarded to ultra-low fluence [44, 149-153], when the concentration of ballistically generated displacements is smaller than that of free charge carriers. This latter method is based on a comparison of depth profiles of displacements predicted by ballistic calculations and measured by deep level transient spectroscopy (DLTS), spreading resistance profiling (SRP), or photoluminescence (PL). However, this approach, although important for understanding defect trapping centers in as-grown $\mathrm{Si}$, is challenging to apply to many cases of practical importance, such as ion implantation doping and nuclear material performance, which involve orders of magnitude larger fluence and/or material systems that are unsuitable for DLTS, SRP, or PL characterization. This method [44, 149-153] is also complicated by a contribution from ion channeling, leading to the appearance of exponentially decaying tails in defect distributions, qualitatively similar to those expected for a process of trap-limited diffusion [152].

In contrast to traditional fluence-rate effect studies, a pulsed ion beam method can be used to separate spatial and temporal information. We have recently demonstrated [158] that the characteristic time constant $\tau$ of DA can be measured directly by studying the dependence of lattice disorder on the time interval of the passive part of the beam cycle, $t_{\text {off }}$. The inset in Fig. 5.11 shows a time dependence of the flux in such pulsed ion beam experiments and defines the pulsing related parameters: $t_{\mathrm{on}}$, $t_{\text {off }}$, and $F_{\text {on }}$. With this method, a $\tau$ of $\sim 6 \mathrm{~ms}$ has been measured for Si irradiated at RT with $500 \mathrm{keV}$ Ar ions, significantly clarifying previous estimates of $\tau$ in RT Si, ranging over 12 orders of magnitude [158].

In this section, we demonstrate how the pulsed beam approach can also be used 
for a direct measurement of the $L_{\mathrm{d}}$ by studying the dependence of lattice disorder on the duration of the active part of the beam cycle, $t_{\mathrm{on}}$. With this method, we obtain a $L_{\mathrm{d}}$ of $\sim 30 \mathrm{~nm}$ for Si crystals bombarded at RT with $500 \mathrm{keV}$ Ar ions.

Float-zone grown (100) Si single crystals with a resistivity of $\sim 5 \Omega \mathrm{cm}$ were bombarded at RT with $500 \mathrm{keV}^{40} \mathrm{Ar}^{+}$ions at $7^{\circ}$ off the [100] direction to minimize channeling effects. The 4 MV ion accelerator (National Electrostatics Corporation, model $4 \mathrm{UH}$ ) at Lawrence Livermore National Laboratory was used for both ion irradiation and ion beam analysis. As described in detail previously [158], in order to avoid complexity related to differences between instantaneous and average fluxs inherent to experiments with rastered ion beams, all irradiations were performed in a broad beam mode. Beam pulsing was achieved by applying high voltage pulses to a pair of plates deflecting the beam off the final beam defining aperture [158]. The total fluence was split into a number of equal pulses with a fluence per pulse of $F_{\text {on }} t_{\text {on }}$ (see the inset in Fig. 5.11). Each sample was irradiated to a total fluence of $2 \times 10^{14} \mathrm{~cm}^{-2}$ with a constant $F_{\text {on }}$ of $\sim 1.6 \times 10^{13} \mathrm{~cm}^{-2} \mathrm{~s}^{-1}$. Such irradiation produces sub-amorphization damage in a non-linear region of the damage buildup curve [158], where DA processes are particularly pronounced [43]. The duration of the passive part of the cycle $\left(t_{\text {off }}\right)$ was kept constant at $100 \mathrm{~ms}$, and the dependence of lattice damage on $t_{\mathrm{on}}$ (varied from 0.5 to $100 \mathrm{~ms}$ ) was studied.

The lattice disorder was measured ex-situ by Rutherford backscattering/channeling (RBS/C) spectrometry with $2 \mathrm{MeV}^{4} \mathrm{He}^{+}$ions incident along the [100] direction and backscattered into a detector at $164^{\circ}$ relative to the incident beam direction. All RBS/C spectra were analyzed with one of the conventional algorithms [70] for extracting the effective number of scattering centers (referred to below as "relative disorder").

Figure 5.11 shows selected depth profiles of lattice disorder in Si bombarded with 
Rev. 1.2

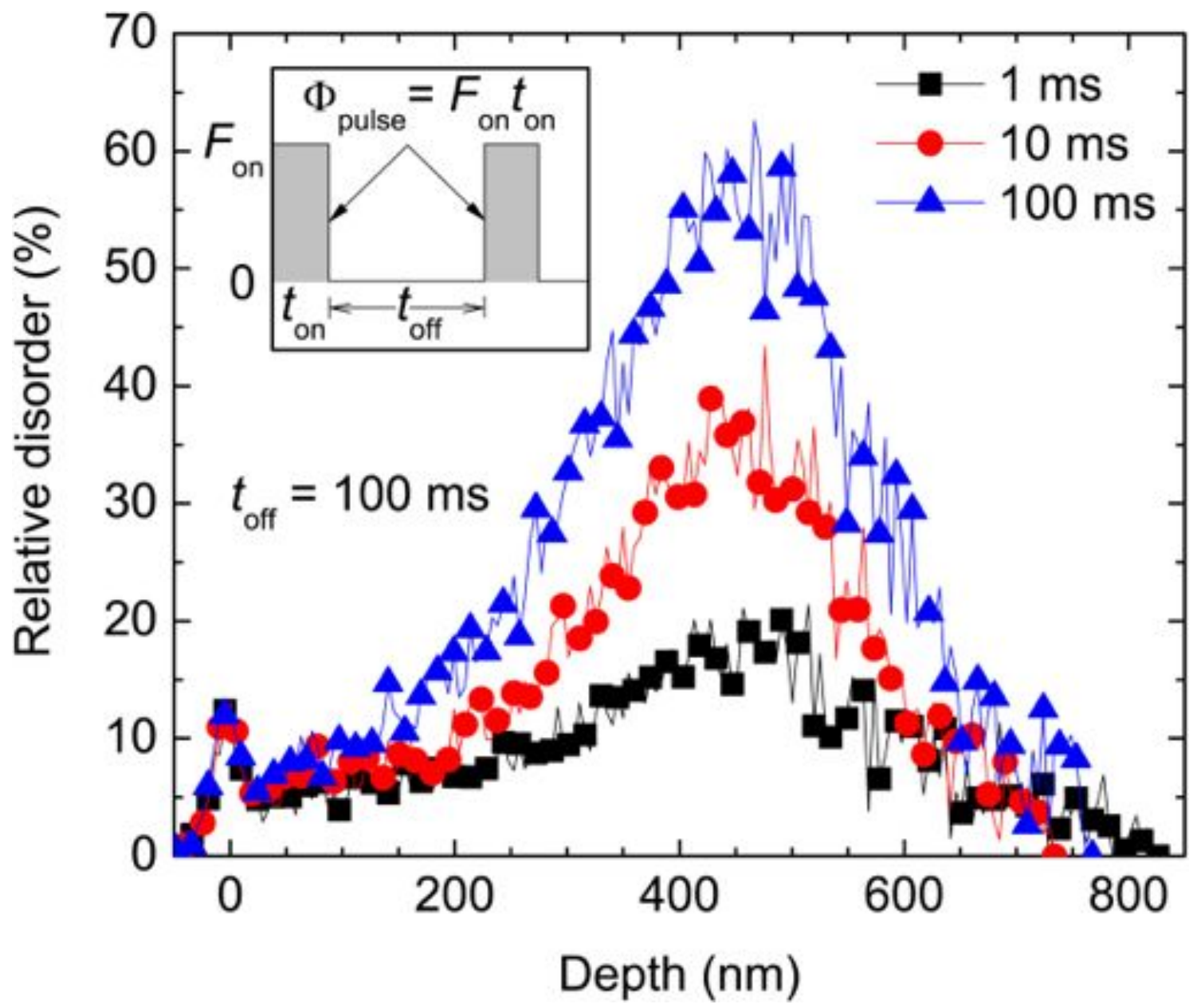

Figure 5.11: Selected depth profiles of relative disorder in Si bombarded at room temperature by $500 \mathrm{keV}$ Ar ions with a pulsed beam with different values of $t_{\mathrm{on}}$ (indicated in the legend) and all the other parameters fixed (total fluence $=2 \times 10^{14}$ $\mathrm{cm}^{-2}, t_{\mathrm{off}}=100 \mathrm{~ms}$, and $F_{\mathrm{on}} \approx 1.6 \times 10^{13} \mathrm{~cm}^{-2} \mathrm{~s}^{-1}$ ). The inset shows a schematic of the time dependence of the flux, defining $t_{\mathrm{on}}, t_{\mathrm{off}}, F_{\text {on }}$ (the maximum instantaneous flux), and $\Phi_{\text {pulse }}$ (the fluence per pulse). 
all the parameters, except for $t_{\text {on }}$, kept constant. The duration of the passive part of the cycle, $t_{\text {off }}=100 \mathrm{~ms}$, was chosen much larger than the defect stabilization time $\tau$, which is $\sim 6 \mathrm{~ms}$ for these conditions [158]. Profiles in Fig. 5.11 reveal two distinct peaks. One peak is positioned at or near the surface. The other, main peak is centered on $\sim 450 \mathrm{~nm}$, which corresponds to the position of the maximum nuclear energy loss of $500 \mathrm{keV}$ Ar ions ( $445 \mathrm{~nm}$ from TRIM code calculations) [20]. Such a bimodal damage accumulation behavior is consistent with a number of previous reports $[39,42,43,138,158]$. It points to an important role of the sample surface in DA (more accurately, the interface between the Si crystal and its native oxide layer). Figure 5.11 also reveals that the amount of stable disorder in the bulk peak increases with increasing $t_{\text {on }}$, while the damage accumulation within $\sim 30 \mathrm{~nm}$ from the sample surface is essentially independent of $t_{\mathrm{on}}$. This observation suggests a difference in mechanisms of DA processes in the crystal bulk and at the surface. It is also consistent with several previous reports [42, 43, 138, 158].

The dependence of the level of maximum bulk disorder on $t_{\text {on }}$ is better illustrated in Fig. 5.12, with error bars corresponding to peak-to-peak noise in RBS/C-derived disorder profiles such as shown in Fig. 5.11. Figure 5.12 clearly shows a trend of increased disorder with increasing $t_{\text {on }}$ above a certain critical value of $\sim 2 \mathrm{~ms}$, which we will refer to as $t_{\mathrm{on}}^{L_{\mathrm{d}}}$. For $t_{\mathrm{on}} \lesssim t_{\mathrm{on}}^{L_{\mathrm{d}}}$, the disorder level is essentially independent of $t_{\text {on }}$ (within experimental error bars). Such a $t_{\text {on }}^{L_{\mathrm{d}}}$ is related to the defect diffusion length, $L_{\mathrm{d}}$. Indeed, $t_{\mathrm{on}}$ determines the fluence delivered in every pulse, $F_{\mathrm{on}} t_{\mathrm{on}}$ (see the inset in Fig. 5.11). Such a fluence per pulse in turn determines the average lateral distance between individual collision cascades for each pulse: $L_{\text {overlap }} \approx 1 / \sqrt{F_{\text {on }} t_{\text {on }}}$. For pulses with small $t_{\text {on }}, L_{\text {overlap }}$ is larger than the average lateral size of damage zones associated with individual collision cascades. As illustrated in the inset of Fig. 5.12, such damage zone sizes are defined by both the size of ballistic cascades 
$\left(R_{\text {ballistic }}\right)$ and the $L_{\mathrm{d}}$. For cases of low $t_{\text {on }}$ and $L_{\text {overlap }}>2\left(L_{\mathrm{d}}+R_{\text {ballistic }}\right)$, mobile defects produced in different cascades cannot interact with each other since $t_{\text {off }} \gg \tau$. Hence, DA processes involving mobile defects generated in different ion pulses are negligible. In this case, the buildup of stable damage is independent of the pulse duration $\left(t_{\text {on }}\right)$, as observed in the experiment (Fig. 5.12).

For $t_{\text {on }} \gtrsim t_{\text {on }}^{L_{\mathrm{d}}}=1 /\left(4 F_{\text {on }}\left(L_{\mathrm{d}}+R_{\text {ballistic }}\right)^{2}\right)$, however, unstable mobile defects produced in adjacent cascades begin to interact. This results in increased stable disorder due to nonlinear defect interaction processes. Various possible defect interaction scenarios could lead to such non-linearity. For example, during the active part of each cycle, the efficiency of stable defect production is different before and during the regime when a stationary population of mobile (unstable) defects is reached. The quantitative dependence of the buildup of stable disorder on $t_{\text {on }}$ is determined by specific defect interaction processes, which are still poorly understood and debatable even for $\mathrm{Si}$ at RT $[39,42,44,137-139,148-154]$. We, however, emphasize that the determination of the $L_{\mathrm{d}}$ based on the threshold value of $t_{\mathrm{on}}$ proposed here does not require the knowledge of these specific defect interaction processes since it is not based on an analysis of the non-linearity of the damage buildup behavior.

From Fig. 5.12, a $t_{\text {on }}^{L_{\mathrm{d}}}$ of $\sim 2$ ms corresponds to a $L_{\mathrm{d}}$ of $\sim 30 \mathrm{~nm}$, given that $R_{\text {ballistic }}$ is only $\sim 1-2 \mathrm{~nm}[20,159]$. This is comparable with several previous estimates of a $L_{\mathrm{d}}$ of $\sim 10-50 \mathrm{~nm}$ with other methods for different types of Si irradiated at RT with ions or electrons $[39,138,139,149,151,153,154]$. Interestingly, a $L_{\mathrm{d}}$ of $\sim 30 \mathrm{~nm}$ is also consistent with a distance from the sample surface where damage is essentially independent of $t_{\text {on }}$ (see Fig. 5.11 and a discussion above), suggesting that the sample surface acts as an efficient sink for mobile defects. A $L_{\mathrm{d}}$ of $\sim 30 \mathrm{~nm}$ is, however, much smaller than a $L_{\mathrm{d}}$ of $\sim 300-2000 \mathrm{~nm}$ estimated in Refs. [149, 150, 153] based on DLTS, SRP, or PL measurements. This discrepancy could be attributed to 


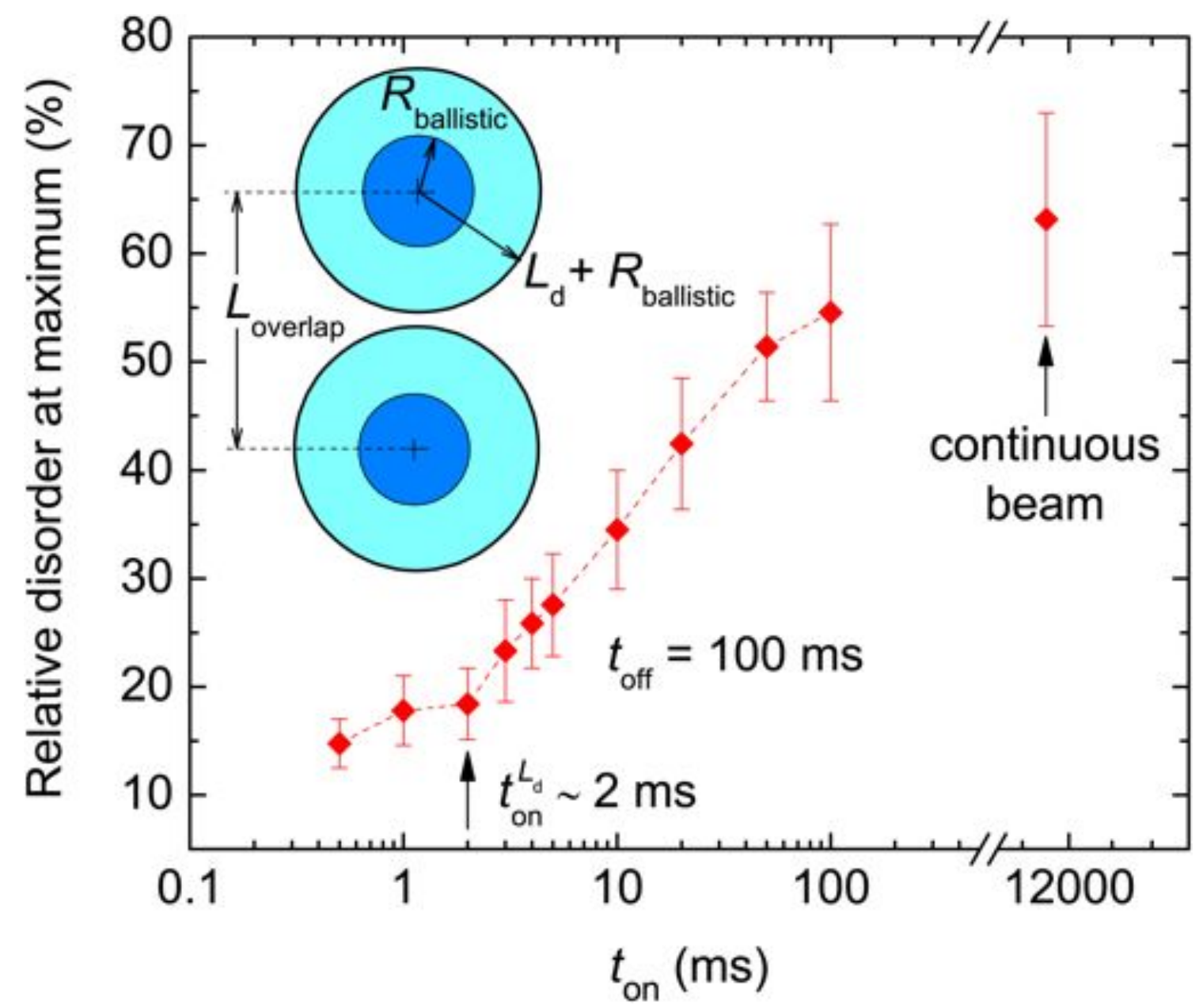

Figure 5.12: Dependence of relative disorder at the maximum of the bulk defect peak in Si bombarded at room temperature by a pulsed beam of $500 \mathrm{keV}$ Ar ions on the duration of the active part of the cycle, $t_{\mathrm{on}}$, and all the other parameters fixed as in Fig. 5.11. The maximum damage level produced by a continuous beam $\left(t_{\mathrm{off}}=0\right)$ is also shown. A critical value of $t_{\text {on }}$ above which the damage level is rapidly increases is labeled as $t_{\mathrm{on}}^{L_{\mathrm{d}}}$ and marked by an arrow. The inset shows a schematic of cascades in a slice made perpendicular to the beam direction, defining parameters $R_{\text {ballistic }}$ (the average radius of ballistic cascades), $L_{\mathrm{d}}$ (the characteristic defect diffusion length), and $L_{\text {overlap }}$ (the average lateral distance between the centers of collision cascades in one pulse). 
channeling effects discussed by Nielsen et al.[152] or to a possible dependence of the $L_{\mathrm{d}}$ on irradiation conditions. Future systematic studies should clarify it.

Our results can also be used to estimate the diffusion coefficient of the defects dominating DA processes. With $\tau=6 \mathrm{~ms}$ (Ref. [158]) and $L_{\mathrm{d}}=30 \mathrm{~nm}, D \approx L_{\mathrm{d}}^{2} / \tau \approx$ $2 \times 10^{-9} \mathrm{~cm}^{2} \mathrm{~s}^{-1}$. Inerestingly, this value is close to the value of the RT diffusion coefficient of vacancies in $\mathrm{Si}\left(\sim 4 \times 10^{-9} \mathrm{~cm}^{2} \mathrm{~s}^{-1}\right)$ [160]. More work is, however, currently needed to ascertain if the primary mobile defect species determining the RT DA processes in ion-bombarded $\mathrm{Si}$ are isolated vacancies $[39,43,138,153]$ rather than other possible defects such as interstitials [44, 150, 151], di-interstitials [161], "bond defects" [162], or some combination of several interacting defect species.

Finally, the method to measure the $L_{\mathrm{d}}$ proposed here could also be applied to test the models of damage buildup in solids, to study the dependence of the $L_{\mathrm{d}}$ on irradiation conditions, and to measure the $L_{\mathrm{d}}$ in other technologically relevant materials. Of particular interest is the knowledge of length scales of DA processes when designing materials with improved "radiation-resistance" via controlled interaction of mobile defects with surfaces and interfaces [28-30]. In this case, the diffusion length determines the required dimensions of "radiation-resistant" nanostructures.

In this section, we have demonstrated an experimental method to measure the effective defect diffusion length $\left(L_{\mathrm{d}}\right)$ after cascade thermalization. Our approach can be summarized as follows: (i) the total ion fluence is chosen in the nonlinear regime of the damage buildup behavior, and $t_{\text {off }} \gg \tau$ is selected; (ii) samples are bombarded with a pulsed ion beam, and the dependence of the level of stable lattice disorder on $t_{\text {on }}$ is measured (with all the other irradiation parameters kept constant); and (iii) the $L_{\mathrm{d}}$ is calculated based on the threshold value of $t_{\text {on }}^{L_{\mathrm{d}}}$ (above which the damage level exhibits a dependence on $\left.t_{\text {on }}\right)$ as $L_{\mathrm{d}} \approx 1 /\left(2 \sqrt{t_{\text {on }}^{L_{\mathrm{d}}} F_{\mathrm{on}}}\right)-R_{\text {ballistic }}$, where the average lateral size of ballistic cascades $\left(R_{\text {ballistic }}\right)$ can be estimated from ballistic calculations 
Rev. 1.2

such as the TRIM code $[20,159]$. With this method, we have measured a $L_{\mathrm{d}}$ of $\sim 30$ nm in Si irradiated at RT with $500 \mathrm{keV}$ Ar ions. 
Rev. 1.2

\section{SUMMARY}

The purpose of the current section is to highlight the major findings of this dissertation. The summary is broken into two portions, the first is related to heavyion-irradiated $\mathrm{ZnO}$ and the second is related to findings associated with pulsed ion beam irradiation.

\subsection{Heavy-ion-bombarded $\mathrm{ZnO}$}

By performing irradiation at two incident ion angles to increasing DPA, we have shown that the formation and propagation of the IP are independent of the length of the radiation damage profile (for the irradiation conditions used here). The sample surface is essential for its formation and existence, which is supported by the fact that both the IP position and intensity are essentially the same for both irradiation angles (to the same DPA) with about a factor of two different sputtering rates. In addition, given the large differences in the spatial separation of ballistically generated vacancies and interstitials between the two irradiation angles, this effect is unlikely to play the dominating role in IP formation.

Cavities are observed in 5 DPA irradiated samples and oxygen loss is observed (by EDS/STEM) in the sample irradiated to 15 DPA. A high local concentration of vacancies after the thermalization of dense collision cascades could lead to the formation of vacancy clusters that, upon continuing irradiation, evolve into cavities. Thermal spikes are also likely to cause material decomposition, accompanied by loss of oxygen from the sample surface and from the walls of cavities. The resultant microstructure at $15 \mathrm{DPA}$ is quite complex and consists of a layered structure with alternating layers of stoichiometric and oxygen deficient $\mathrm{ZnO}$. The cavities observed at 5 DPA are no longer present at $15 \mathrm{DPA}$, and the IP has moved into the crystal 
bulk. The IP observed in RBS/C (in particular for 15 DPA) has been correlated to STEM/EDS images which show that it is a Zn-rich defect band. The surface may act as an efficient defect sink resulting in a near-surface gradient of instantaneous concentrations of mobile defects during irradiation. A concentration gradient could drive defect diffusion toward the surface and result in the movement of the IP (i.e., the Zn-rich defect band). In addition, it is speculated that the IP layer itself could promote defect recombination. This is due to the observation of the formation of an abrupt step between the IP and the BP in the depth distribution of stable lattice disorder. The IP movement continues ( $\sim$ a few nm/DPA) until it reaches a distance from the sample surface comparable with what we describe as the effective defect diffusion length. Indeed, the IP moves to a depth where the defect concentration gradient is thought to diminish, at $\sim 30-40 \mathrm{~nm}$ from the sample surface.

Given such a strong effect of the free surface on radiation damage in (0001) $\mathrm{ZnO}$, we performed another experiment to determine if damage buildup can be controlled by manipulating the surface properties of (0001) ZnO. We have shown that nearsurface damage in (0001) $\mathrm{ZnO}$ can be suppressed by placing a thin layer of $\mathrm{AlO}(\mathrm{OH})$ on the sample surface which prevents the formation of nanocavities and reduce the level of stable disorder up to depths of $\sim 100 \mathrm{~nm}$ from the surface. The suppression of near-surface damage (including both BP and IP as well as the formation of nanocavities) could be related to several complex phenomena, and our current data is insufficient to differentiate between all the scenarios.

It has been previously revealed that variations exist in the DA efficiency among different crystallographic orientations under irradiation. That is, certain orientations experience increased levels of disorder compared to other orientations irradiated under the same conditions. It has been suggested that either enhanced DA due to the ease of point defect migration along certain crystallographic orientations (i.e. 
enhanced diffusion), the stability of extended defects along certain crystallographic orientations, or variations in the surface trapping efficiency are likely responsible. Additionally, a large dependence on the irradiation spectrum has been observed for the resultant level of disorder. There is strong evidence supporting the existence of defects with varied charged states with the resultant defect charge state being strongly correlated to the irradiation spectrum. Ionized point defects in semiconductors and insulators may have a lower barrier to migration than that of their non-ionized counterparts. The formation, stability and diffusion of multiple defect charge states, dependent on the irradiation spectrum, coupled with a polar termination that exhibits a net surface charge may act to substantially alter defect diffusion. DA processes are likely to depend, not only on the defect charge state, but also on the crystallographic orientation and the diffusional pathways to the free surface. Additionally, the trapping efficiency at the surface may be highly dependent on the net charge on the surface and the dominant defect charge state interacting with the surface.

Given the large role of the free surface in IP formation in $\mathrm{ZnO}$, it is reasonable to question whether all free surfaces will behave similarly, or whether the polar free surface is an exception. For the next experiment, $\mathrm{ZnO}$ crystals of various orientations are simultaneously bombarded with heavy ions. It is observed that non-polar $(11 \overline{2} 0$ and 1010) surface terminated $\mathrm{ZnO}$ exhibits enhanced radiation tolerance as compared to polar (0001) terminated surfaces. These other orientations also do not show the formation of an IP which lends credence to the notion that the polar free surface (0001) plays a vital role in IP formation, defect diffusion, and DA under irradiation. We find that DA rates differ substantially among the various orientations and the role of charged defect production and diffusion under irradiation may be significant. DA anisotropy and ionization enhanced diffusion provide possible explanations for 
the phenomena which is observed in $\mathrm{ZnO}$. Cascade density effects (light ion versus heavy ion) have been reported in $\mathrm{ZnO}$. The charge state, stability and diffusivity of defects nucleated out of dense cascades may differ substantially from that of light ion irradiation. Higher charge state defects may experience an enhanced rate of diffusion toward the polar surface due to the electrostatic potential present at the sample surface. This may explain differences observed in heavy ion irradiated polar and non-polar surface terminated $\mathrm{ZnO}$, however, more experiments are clearly needed to fully explain such complex behavior.

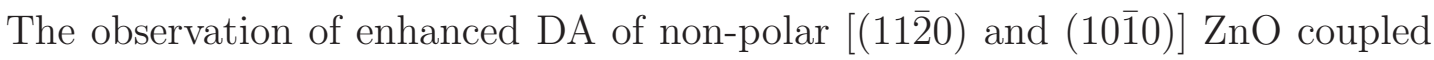
with the previous report that a thin film placed on the polar (0001) surface dramatically reduces radiation damage buildup is intriguing. In the final experiment on $\mathrm{ZnO}$, we simultaneously bombard $\mathrm{ZnO}$ crystals (both polar and non-polar orientations, with and without $\mathrm{Al}_{2} \mathrm{O}_{3}$ surface modification) with $500 \mathrm{keV}$ Xe ions and observe that, although surface modification completely suppresses the formation of an IP in polar $\mathrm{ZnO}$ and reduces disorder in the bulk, it has a negligible effect on bulk radiation damage buildup properties in non-polar films. Disorder levels at the $\mathrm{ZnO} / \mathrm{Al}_{2} \mathrm{O}_{3}$ interface are greater for surface modified non-polar films (being higher than unmodified samples), however, at depths just below the $\mathrm{ZnO} / \mathrm{Al}_{2} \mathrm{O}_{3}$ interface, disorder levels are reduced for surface modified samples compared to those which were unmodified.

\subsection{Pulsed-ion-beam irradiation}

We have derived the theoretical framework and developed an experimental pulsed ion beam method to measure characteristic time constants $(\tau)$ and diffusion lengths $\left(L_{\mathrm{d}}\right)$ of DA processes in solids under irradiation. The experimental apparatus was described in detail along with the pertinent details to replicate the exact system 
used in this dissertation. To perform actual pulsed ion beam experiments, a material which has a wide body of literature was used (i.e Si). To maximize the possibility of measuring time constants associated with DA, a total ion dose was chosen in the nonlinear regime of the damage buildup behavior where DA processes are particularly pronounced.

For the first set of experiments, we measured a dependence of lattice damage on the duration of the passive part of the ion beam cycle with all the other irradiation parameters kept constant. For silicon bombarded with $500 \mathrm{keV}$ Ar ions at RT, the DA time constant is found to be $\sim 6 \mathrm{~ms}$ and the defect relaxation behavior suggests a second order kinetic process.

For the second set of experiments, we measured a dependence of lattice damage on the duration of the active part of the ion beam cycle with all the other irradiation parameters kept constant. With this method, an $L_{\mathrm{d}}$ is calculated based on the observation of a threshold value of $t_{\text {on }}$ above which the damage level exhibits a dependence on $t_{\mathrm{on}}$. With this method, we have measured a $L_{\mathrm{d}}$ of $\sim 30 \mathrm{~nm}$ in $\mathrm{Si}$ irradiated at RT with $500 \mathrm{keV}$ Ar ions.

Future pulsed beam irradiation experiments should gain insight into how the DA time constant $\tau$ in $\mathrm{Si}$ depends on the type and concentration of dopants and on irradiation parameters. Data obtained with this method could also have important implications for the development of physically sound models of damage accumulation in solids. Clearly, a successful model for Si should include specific defect interaction processes with characteristic relaxation times and kinetics revealed by this work. In addition, this pulsed-beam method could also be applied to study defect dynamics in technologically relevant materials other than Si. 
Rev. 1.2

\section{REFERENCES}

[1] C. Jagadish and S. Pearton, eds., Zinc Oxide Bulk, Thin Films and Nanostructures (Elsevier Amsterdam, 2006).

[2] S. O. Kucheyev and C. Jagadish, Chapter 8 in C. Jagadish and S. Pearton, eds., Zinc Oxide Bulk, Thin Films and Nanostructures (Elsevier Amsterdam, 2006), p. 285.

[3] K. Lorenz, E. Alves, E. Wendler, O. Bilani, W. Wesch, and M. Hayes, Appl. Phys. Lett. 87, 191904 (2005).

[4] A. Yu. Azarov, S. O. Kucheyev, A. I. Titov, and P. A. Karaseov, J. Appl. Phys. 102, $083547(2007)$.

[5] E. Wendler, O. Bilani, K. Gartner, W. Wesch, M. Hayes, F. Auret, K. Lorenz, and E. Alves, Nucl. Instr. Methods Phys. Res. B 267, 2708 (2009).

[6] A. Yu. Azarov, A. I. Titov, P. A. Karaseov, S. O. Kucheyev, A. Hallen, A. Yu. Kuznetsov, B. G. Svensson, and A. P. Pathak, Vacuum 84, 1058 (2010).

[7] A. Yu. Azarov, B. G. Svensson, A. Hallen, X. L. Du, and A. Yu. Kuznetsov, J. Appl. Phys. 108, 033509 (2010).

[8] K. Lorenz, M. Peres, N. Franco, J. G. Marques, S. M. C. Miranda, S. Magalhaes, T. Monteiro, W. Wesch, E. Alves, and E. Wendler, Proc. SPIE 7940, $79400 \mathrm{O}$ (2011).

[9] V. A. Coleman, H. H. Tan, C. Jagadish, S. O. Kucheyev, and J. Zou, Appl. Phys. Lett. 87, 231912 (2005). 
[10] G. Perillat-Merceroz, P. Gergaud, P. Marotel, S. Brochen, P. H. Jouneau, and G. Feuillet, J. Appl. Phys. 109, 023513 (2011).

[11] M. T. Myers, S. Charnvanichborikarn, L. Shao, and S. O. Kucheyev, Script. Mater. 67, 65 (2012).

[12] M. T. Myers, S. Charnvanichborikarn, C. C. Wei, Z. P. Luo, A. Aitkaliyeva, L. Shao, and S. O. Kucheyev, Acta Mater. 60, 6086 (2012).

[13] S. O. Kucheyev, J. S. Williams, C. Jagadish, J. Zou, C. Evans, A. J. Nelson, and A. V. Hamza, Phys. Rev. B 67, 094115 (2003).

[14] E. Simonen, J. Nucl. Mater. 117, 113 (1983).

[15] N. M. Ghoniem, J. Nucl. Mater. 89, 359 (1980).

[16] Michael Nastasi, James W. Mayer, and James K. Hirvonen. Ion-Solid Interactions. (Cambridge University Press, 1996).

[17] J. R. Bird and J. S. Williams, Ion Beams for Materials Analysis. (Academic Press Australia, 1989).

[18] J. W. Mayer and E. Rimini, Ion Beam Handbook for Material Analysis. (Academic Press Inc, New York, 1977).

[19] G. S. Was, Fundamentals of Radiation Materials Science, (Springer, Heidelberg, Germany, 2007).

[20] J. Ziegler, J. Biersack, and U. Littmark, The Stopping and Range of Ions in Solids, Vol. 1 (Pergamon, New York, 1985).

[21] O. B. Firsov, Sov. Phys. JETP 36, 1076 (1959). 
Rev. 1.2

[22] G. W. Kinchin and R. S. Pease, Rep. Prog. Phys. 18, 1 (1955).

[23] J. A. Brinkman, Amer. J. of Phys. 24, 246 (1956).

[24] J. S. Williams, Rep. Prog. Phys. 49, 491 (1986).

[25] E. Chason, S. T. Picraux, J. M. Poate, J. O. Borland, M. I. Current, T. Diaz de la Rubia, D. J. Eaglesham, O. W. Holland, M. E. Law, C. W. Magee, J. W. Mayer, J. Melngailis, and A. F. Tasch, J. Appl. Phys. 81, 6513 (1997).

[26] M. T. Robinson, J. Nucl. Mater. 216, 1 (1994); and references therein.

[27] W. J. Weber, R. C. Ewing, C. R. A. Catlow, T. Diaz de la Rubia, L. W. Hobbs, C. Kinoshita, Hj. Matzke, A. T. Motta, M. Nastasi, E. K. H. Salje, E. R. Vance, and S. J. Zinkle, J. Mater. Res. 13, 1434 (1998).

[28] X.-M. Bai, A. F. Voter, R. G. Hoagland, M. Nastasi, and B. P. Uberuaga, Science 327, 1631 (2010).

[29] E. G. Fu, A. Misra, H. Wang, L. Shao, and X. Zhang, J. Nucl. Mater. 407, 178 (2010).

[30] S. Charnvanichborikarn, M. T. Myers, L. Shao, and S. O. Kucheyev, Scripta Mater. 67, 205 (2012).

[31] Y. Guerin, G. S. Was, and S. J. Zinkle, MRS Bull. 34, 10 (2009).

[32] H. M. Naguib and R. Kelly, Radiat.. Eff. 25, 1 (1975).

[33] F. F. Morehead and B. L. Crowder, Radiat. Eff. 6, 27 (1970).

[34] J. F. Gibbons, P. IEEE 60, 1062 (1972).

[35] F. F. Morehead, J. Appl. Phys. 43, 1112 (1972). 
Rev. 1.2

[36] G. Bai and M.-A. Nicolet, J. Appl. Phys. 70, 3551 (1991).

[37] P. Sigmund, I. S. Bitensky, and J. Jensen, Nucl. Instr. Methods Phys. Res. B 112, 1 (1996).

[38] A. I. Titov, S. O. Kucheyev, V. S. Belyakov, and A. Yu. Azarov, J. Appl. Phys. 90, 3867 (2001).

[39] A. I. Titov, A. Yu. Azarov, L. M. Nikulina, and S. O. Kucheyev, Phys. Rev. B 73, $064111(2006)$.

[40] G. Carter, Radiat. Eff. 100, 281 (1987).

[41] G. Carter, I. V. Katardjiev, and M. J. Nobes, Radiat. Eff. 105, 211 (1988).

[42] S. A. R. Al-Hashmi and G. Carter, Radiat. Eff. 102, 83 (1987).

[43] A. I. Titov and G. Carter, Nucl. Instr. Methods Phys. Res. B 119, 491 (1996).

[44] B. G. Svensson, C. Jagadish, and J. S. Williams, Phys. Rev. Lett. 71, 1860 (1993).

[45] J. Lindhard. Kgl. Dan. Vidensk. Selsk. Mat. Fys. Medd. 34, 14 (1965).

[46] P. J. Goodhew, F. J. Humphreys, and R. Beanland, Electron Microscopy and Analysis. (Taylor \& Francis, New York, 2000).

[47] D. B. Williams and C. B. Carter, Transmission Electron Microscopy A Textbook for Materials Science. 2nd ed. (Springer, New York, 2008).

[48] Hj. Matzke, Radiat.. Eff. 64, 3 (1982).

[49] R. C. Ewing, W. J. Weber, F. W. Clinard Jr., Prog. Nucl. Energy 29, 63 (1995). 
Rev. 1.2

[50] S. J. Zinkle, C. Kinoshita, J. Nucl. Mater. 251, 200 (1997).

[51] C. J. McHargue, Mater. Sci. Eng. A 253, 94 (1998).

[52] C. W. White, L. A. Boatner, P. S. Sklad, C. J. McHargue, J. Rankin, G. C. Farlow, M. J. Aziz, Nucl. Instr. Methods Phys. Res. B 32, 11 (1988).

[53] L. W. Hobbs, F.W. Clinard, S. J. Zinkle, and R. C. Ewing, J. Nucl. Mater. 216, 291 (1994).

[54] C. W. White, C. J. McHargue, P. S. Sklad, L. A. Boatner, and G. C. Farlow, Mater. Sci. Rep. 4, 41 (1989); and references therein.

[55] S. J. Zinkle, Radiat.. Eff. 148, 447 (1999).

[56] S. J. Zinkle, Nucl. Instrum. Methods Phys. Res. B 116, 92 (1996).

[57] J. C. Bourgoin and J. W. Corbett, Phys. Lett. 38A, 2 (1972).

[58] J. C. Bourgoin and J. W. Corbett, Radiat. Eff. 36, 157 (1978).

[59] D. G. Walker, J. Nucl. Mater. 14, 195 (1964).

[60] H. Abe, C. Kinoshita, P. R. Okamoto, and L. E. Rehn, J. of Nucl. Mater. 212, 298 (1994).

[61] J. Goniakowski, F. Finocchi, and C. Noguera, Rep. Prog. Phys. 71, 016501 (2008); and references therein.

[62] S. O. Kucheyev, J. S. Williams, and S. J. Pearton, Mater. Sci. Eng., R 33, 51 (2001); and references therein.

[63] H. H. Tan, J. S. Williams, J. Zou, D. J. H. Cockayne, S. J. Pearton, and R. A. Stall, Appl. Phys. Lett. 69, 2364 (1996). 
[64] C. Liu, B. Mensching, M. Zeitler, K. Volz, and B. Rauschenbach, Phys. Rev. B 57, 2530 (1998).

[65] S. O. Kucheyev, J. S. Williams, C. Jagadish, G. Li, and S. J. Pearton, Appl. Phys. Lett. 76, 3899 (2000).

[66] S. O. Kucheyev, J. S. Williams, and S. J. Pearton, Mater. Sci. Eng., R 33, 51 (2001).

[67] M. D. McCluskey and S. J. Jokela, J. Appl. Phys. 106, 071101 (2009); and references therein.

[68] E. Sonder, R. A. Zuhr, and R. E. Valiga, J. Appl. Phys. 64, 1140 (1988).

[69] D. C. Look, G. Farlow, P. Reunchan, S. Limpijumnong, S. Zhang, and K. Nordlund, Phys. Rev. Lett. 9522 (2005).

[70] K. Schmid, Radiat. Eff. 17, 201 (1973).

[71] B. R. Appleton and L. C. Feldman, J. Phys. Chem. of Solids 33, 507 (1972).

[72] I. Stensgaard, L. C. Feldman, and P. J. Silverman, Surf. Sci. 77, 513 (1978).

[73] E. Horvath, A. Nemeth, A. A. Koos, M. C. Bein, A. L. Toth, Z. E. Horvath, L. P. Biro, and J. Gyulai, Superlattice. Microst. 42, 392 (2007).

[74] D. R. Baer, M. H. Engelhard, A. S. Lea, P. Nachimuthu, T. C. Droubay, J. Kim, B. Lee, C. Mathews, R. L. Opila, L. V. Saraf, W. F. Stickle, R. M. Wallace, and B. S. Wright, J. Vac. Sci. Technol. A 28, 1060 (2010).

[75] P. Sigmund, Phys. Rev. 184, 383 (1969). 
[76] Y. Zhang, W. Weber, W. Jiang, V. Shutthanandan, S. Thevuthasan, M. Janson, and A. Hallen, Nucl. Instr. Methods Phys. Res. B 219-220, 647 (2004).

[77] H. Matzke, A. Turos, and G. Linker, Nucl. Instr. Methods Phys. Res. B 91, 294 (1994).

[78] C. W. Rischau, C. S. Schnohr, E. Wendler, and W. Wesch, J. Appl. Phys. 109, 113531 (2011).

[79] A. Turos, S. Fritz, and H. Matzke, Phys. Rev. B 41, 3968 (1990).

[80] J. A. Edmond, R. F. Davis, S. P. Withrow, and K. L. More, J. Mater. Res. 3, 321 (1988).

[81] W. J. Weber, N. Yu, L. M. Wang, and N. J. Hess, J. Nucl. Mater. 244, 258 (1997).

[82] W. J Weber, N. Yu and L. M. Wang, J. Nucl. Mater. 253, 53 (1998).

[83] O. W. Holland, J. D. Budai, and C. W. White, Appl. Phys. Lett. 57, 243 (1990).

[84] Hj. Matzke and L. M. Wang, J. Nucl. Mater. 231, 155 (1996).

[85] R. W. Cahn, J. Inst. Met. 76, 121 (1949); J. F. Nye, Acta Metallurgica 1, 153 (1953).

[86] J. J. Gilman, Acta Metallurgica 3, 277 (1955).

[87] D. A. Thompson, Radiat.. Eff. 56, 105 (1981).

[88] Z. Q. Chen, M. Maekawa, S. Yamamoto, A. Kawasuso, X. L. Yuan, T. Sekiguchi, R. Suzuki, and T. Ohdaira, Phys. Rev. B 69, 035210 (2004). 
[89] A. Zubiaga, F. Tuomisto, V. A. Coleman, H. H. Tan, C. Jagadish, K. Koike, S. Sasa, M. Inoue, and M. Yano, Phys. Rev. B 78, 035125 (2008).

[90] G. Perillat-Merceroz, P. Gergaud, P. Marotel, S. Brochen, P. H. Jouneau, and G. Feuillet, J. Appl. Phys. 109, 023513 (2011).

[91] S. M. George, Chem. Rev. 110, 111 (2010).

[92] G. P. Pells and M. J. Murphy, J. Nucl. Mater. 191, 621 (1992).

[93] R. Brenier, B. Canut, S. M. M. Ramos, and P. Thevenard, Nucl. Instr. Methods Phys. Res. B 90, 339 (1994).

[94] Y. Chen, D. M. Bagnall, H. Koh, K. Park, K. Hiraga, Z. Zhu, and T. Yao, J. Appl. Phys. 84, 3912 (1998).

[95] C. Noguera, J. Phys.: Condens. Matter 12, R367 (2000).

[96] F. Claeyssens, C. L. Freeman, N. L. Allan, Y. Sun, M. N. R. Ashfold, and J. H. Harding, J. Mater. Chem. 15, 139 (2005).

[97] T. Takeuchi, S. Sota, M. Katsuragawa, M. Komori, H. Takeuchi, H. Amano, and I. Akasaki, Jpn. J. Appl. Phys., Part 2 36, L382 (1997).

[98] D. A. B. Miller, D. C. Chemla, T. C. Damen, A. C. Grossard, W. Wiegmann, T. H. Wood, and C. A. Burrus, Phys. Rev. B 32, 1043 (1985).

[99] M. Leroux, N. Grandjean, M. Laugt, J. Massies, B. Gil, P. Lefebvre, and P. Bigenwald, Phys. Rev. B 58, R13371 (1998).

[100] J. S. Im, H. Kollmer, J. Off, A. Sohmer, F. Scholz, and A. Hangleiter, Phys. Rev. B 57, R9435 (1998). 
[101] J. W. Lee, J. H. Kim, S. K. Han, S. K. Hong, J. Y. Lee, S. I. Hong, T. Yao, J. Cryst. Growth 312, 238 (2010).

[102] S. Yamauchi, H. Handa, A. Nagayama, and T. Hariu, Thin Solid Films 345, $12(1999)$.

[103] S. K. Han, S. K. Hong, J. W. Lee, J. Y. Lee, J. H. Song, Y. S. Nam, S. K. Chang, T. Minegishi, and T. Yao, J. Cryst. Growth 309, 121 (2007).

[104] J. W. Lee, S. K. Han, S. K. Hong, J. Y. Lee, and T. Yao, J. Cryst. Growth 310, $4102(2008)$.

[105] M. C. Schmidt, K. -C. Kim, H. Sato, N. Fellows, H. Masui, S. Nakamura, S. P. Denbaars, and J. S. Speck, Jpn. J. Appl. Phys. 46, L126 (2007).

[106] N. Catarino, E. Nogales, N. Franco, V. Darakchieva, S. M. C. Miranda, B. Mendez, E. Alves, J. G. Marques and K. Lorenz, Europhys. Lett. 97, 68004 (2012).

[107] I. O. Usov, P. N. Arendt, J. R. Groves, L. Stan, and R. F. DePaula, Nucl. Instr. Methods Phys. Res. B 240, 661 (2005).

[108] I. O. Usov, P. N. Arendt, J. R. Groves, L. Stan, and R. F. DePaula, Nucl. Instr. Methods Phys. Res. B 243, 87 (2006).

[109] C. J. McHargue, E. Alves, M. F. da Silva, and J. C. Soares, Nucl. Instr. Methods Phys. Res. B 148, 730 (1999).

[110] G. C. Farlow, P. S. Sklad, C. W. White, C. J. McHargue, and B. R. Appleton, MRS Proceed. 60, 387 (1986). 
Rev. 1.2

[111] M. E. O'Hern, C. J. McHargue, C. W. White, and G. C. Farlow, Nucl. Instr. Methods Phys. Res. B 46, 171 (1990).

[112] R. Brenier, B. Canut, L. Gea, S. M. M. Ramos, P. Thevenard, J. Rankin, L. Romana, and L. A. Boatner, Nucl. Instr. Methods Phys. Res. B 80, 1210 (1993).

[113] G. B. Kreft, J. Vac. Sci. Technol. 14, 533 (1977).

[114] A. Stukowski, Modeling Simul. Mater. Sci. Eng. 18, 015012 (2010).

[115] M. A. Myers, M. T. Myers, M. J. General, J. H. Lee, L. Shao, and H. Wang, Appl. Phys. Lett. 101112101 (2012).

[116] M. T. Myers, S. Charnvanichborikarn, M. A. Myers, J. H. Lee, H. Wang, M. M. Biener, L. Shao, and S. O. Kucheyev, Unpublished (Appl. Surf. Sci.).

[117] M. T. Myers, S. Charnvanichborikarn, L. Shao, and S. O. Kucheyev, Unpublished (PRB).

[118] J. A. Sprague and F. A. Smidt, NRL Memorandum Reports 2555 (p. 25) and 2629 (p. 27), Naval Research Laboratory (1972 \& 1973).

[119] J. L. Brimhall, L. A. Charlot, and E. P. Simonen, J. Nucl. Mater. 103 \& 104, $1147(1981)$.

[120] J. L. Brimhall, E. P. Simonen and L. A. Charlot, J. Nucl. Mater. 117, 118 (1983).

[121] J. L. Brimhall, E. P. Simonen and L. A. Charlot, J. Nucl. Mater. 122 \& 123, 579 (1984).

[122] D. Kaletta, J. Nucl. Mater. 85 \& 86, 775 (1979). 
Rev. 1.2

[123] R. W. Powell and G. R. Odette, J. Nucl. Mater. 85 \& 86, 695 (1979).

[124] N. H. Packan, J. Nucl. Mater. 103 \& 104, 1029 (1981).

[125] E .H. Lee, N. H. Packan, and L. K. Mansur, J. Nucl. Mater. 117, 123 (1983).

[126] N. H. Packan, J. Nucl. Mater. 122 \& 123, 644 (1984).

[127] N. M. Ghoniem, J. Nucl. Mater. 89, 359 (1980).

[128] E. P. Simonen, N. M. Ghoniem, and N. H. Packan, J. Nucl. Mater. 122 \& 123, 391 (1984).

[129] J. Linnros and G. Holmén, J. Appl. Phys. 62, 4737 (1987).

[130] J. Linnros, W. L. Brown, and R. G. Elliman, Mater. Res. Symp. Proc. 100, 369 (1988).

[131] J. Linnros, R. G. Elliman, and W. L. Brown, J. Mater. Res. 3, 1208 (1988).

[132] L. Pelaz, L. A. Marques, and J. Barbolla, J. Appl. Phys. 96, 5947 (2004).

[133] H. J. Stein, J. Appl. Phys. 14, 328 (1969).

[134] K. R. C. Mok, F. Benistant, M. Jaraiz, J. E. Rubio, P. Castrillo, R. Pinacho, and M. P. Srinivasan. J. Appl. Phys. 103, 014911 (2008).

[135] M. Posselt, L. Bischoff, and J. Teichert, Appl. Phys. Lett. 79, 1444 (2001).

[136] B. G. Svensson, C. Jagadish and J. S. Williams, Phys. Rev. Lett. 71, 1860 (1993).

[137] L. T. Chadderton, Radiat. Eff. 8, 77 (1971). 
[138] A. I. Titov, V. S. Belyakov, and A. Yu. Azarov, Nucl. Instrum. Methods Phys. Res. B 212, 169 (2003).

[139] M.-O. Ruault, J. Chaumont, and H. Bernas, Nucl. Instrum. Methods Phys. Res. B 209/210, 351 (1983).

[140] M.-J. Caturla, T. Díaz de la Rubia, L. A. Marqués, and G. H. Gilmer, Phys. Rev. B 54, 16683 (1996).

[141] H. Hensel and H. M. Urbassek, Phys. Rev. B 57, 4756 (1998).

[142] K. Nordlund, M. Ghaly, R. S. Averback, M. Caturla, T. Diaz de la Rubia, and J. Tarus, Phys. Rev. B 57, 7556 (1998).

[143] P. J. Schultz, C. Jagadish, M. C. Ridgway, R. G. Elliman, and J. S. Williams, Phys. Rev. B 44, 9118 (1991).

[144] E. C. Baranova, V. M. Gusev, W. V. Martynenko, C. V. Starinin, and I. B. Haibullin, Radiat. Eff. 18, 21 (1973).

[145] M. L. Swanson, J. R. Parsons, and C. W. Hoelke, Radiat. Eff. 9, 249 (1971).

[146] G. Carter, J. Appl. Phys. 79, 8285 (1996); and references therein.

[147] S. O. Kucheyev, A. Yu. Azarov, A. I. Titov, P. A. Karaseov, and T. M. Kuchumova, J. Phys. D: Appl. Phys. 42, 085309 (2009); and references therein.

[148] Y. Morikawa, K. Yamamoto, and K. Nagami, Appl Phys. Lett. 36, 997 (1980).

[149] J. L. Benton, B. E. Weir, D. J. Eaglesham, R. A. Gottscho, J. Michel, and L. C. Kimerling, J. Vac. Sci. Technol. B 10, 540 (1992). 
[150] K. Kyllesbech Larsen, V. Privitera, S. Coffa, F. Priolo, S. U. Campisano, and A. Carnera, Phys. Rev. Lett. 76, 1493 (1996).

[151] T. Matsukawa, K. Yokoyama, S. Sawara, and I. Ohdomari, Appl. Phys. Lett. 74, 2663 (1999).

[152] H. Kortegaard Nielsen, B. G. Svensson, J.-J. Goubet, and A. Nylandsted Larsen, Appl. Phys. Lett. 82, 3865 (2003).

[153] P. N. K. Deenapanray, Appl. Phys. Lett. 80, 1577 (2002).

[154] G. Lulli, P. G. Merli, and M. Vittori Antisari, Phys. Rev. B 36, 8038 (1987).

[155] H. Strack, J. Appl. Phys. 34, 2405 (1963).

[156] B. Domeij, F. Brown, J. A. Davies, G. R. Piercy, and E. V. Kornelsen, Phys. Rev. Lett. 12, 363 (1964).

[157] R. L. Minear, D. G. Nelson, and J. F. Gibbons, J. Appl. Phys. 43, 3468 (1972).

[158] M. T. Myers, S. Charnvanichborikarn, L. Shao, and S. O. Kucheyev, Phys. Rev. Lett. 109, 095502 (2012).

[159] A. I. Titov, V. S. Belyakov, and S. O. Kucheyev, Nucl. Instrum. Methods Phys. Res. B 194, 323 (2002).

[160] G. S. Oehrlein, I. Krafcsik, J. L. Lindstrom, A. E. Jaworowski, and J. W. Corbett, J. Appl. Phys. 54, 179 (1983).

[161] G. H. Gilmer, T. Diaz de la Rubia, D. M. Stock, and M. Jaraiz, Nucl. Instrum. Methods Phys. Res. B 102, 247 (1995). 
Rev. 1.2

[162] L. A. Marqués, L. Pelaz, M. Aboy, L. Enríquez, and J. Barbolla, Phys. Rev. Lett. 91, 135504 (2003). 
Rev. 1.2

\section{APPENDIX A}

\section{DECHANNELING CALCULATION CODE}

Throughout this dissertation, extracted RBS/C spectra are used to determine the amount of relative disorder in damage-depth profiles. To assist in processing these spectra, the following program was written in Perl (utilizing the PDL data language libraries) to calculate relative disorder as a function of depth given two input files, an aligned RBS/C spectrum and a random RBS/C spectrum. The code is based on the algorithm developed by Schmid [70] which iteratively solves for the dechanneling cross section given certain input parameters. The one main modification that was made to the algorithm is related to the random spectrum. Instead of using a straight line approximation based on the yield at the sample surface for the random level, the actual random spectrum is fitted with a third order polynomial and the iterative subtraction of the dechanneling component is performed against the fitted curve.

The code is called from a standard terminal on any system which is capable of executing Perl scripts by typing,

schmid.pl --a 167 --b 275 --r_fname random.txt --r_low 50 --r_high 260 --r_preset 1000 --s_preset 2000 spectrum.txt

The following defines the parameters that are used in the extraction of the spectrum:

$--\mathrm{a}$

This defines the last channel which defines the bulk damage peak. In the damagedepth profile, this should be the channel which damage is no longer observed. 
This defines the first channel which defines the surface peak or disorder. In the damage-depth profile, this should be the channel associated with the expected sample surface.

$--r \_f$ name

This is the name of the text file which contains the random spectrum.

$--r_{-}$low

This is the lowest channel to which a third order polynomial should be fit to the random spectrum. A lower bound is set to eliminate the low energy noise that plagues very low channel numbers.

$--r \_h i g h$

This is the highest channel to which a third order polynomial should be fit to the random spectrum. An upper bound is set to precisely define the sample surface and to ensure fitting only occurs on the actual spectrum and not on channels which do not correspond to the random spectrum.

$--r_{-}$preset

-- s_preset

These two values account for differences in the total accumulation time between the random spectrum and the aligned spectrum. If the two are the same, then each should be set to identical values. However, if the acquisition time for a random spectrum is lower than that of the aligned spectrum, this should be accounted for in the preset values. 
The final input is given simply as the filename which contains the aligned spectrum. Once the program executes successfully, a log file will be written which contains the relevant parameters for the fitting and the iterative calculation of the dechanneling cross section. Finally, an output file is created with the same name as the original aligned spectrum, however .schmid is appended. For example, if the file spectrum.txt was fed as an input file, the output file which contains the extracted profile would be spectrum.txt.schmid.out. In order to convert this profile from relative disorder to defect concentration, the relative disorder value should be multiplied by the atomic density of the target.

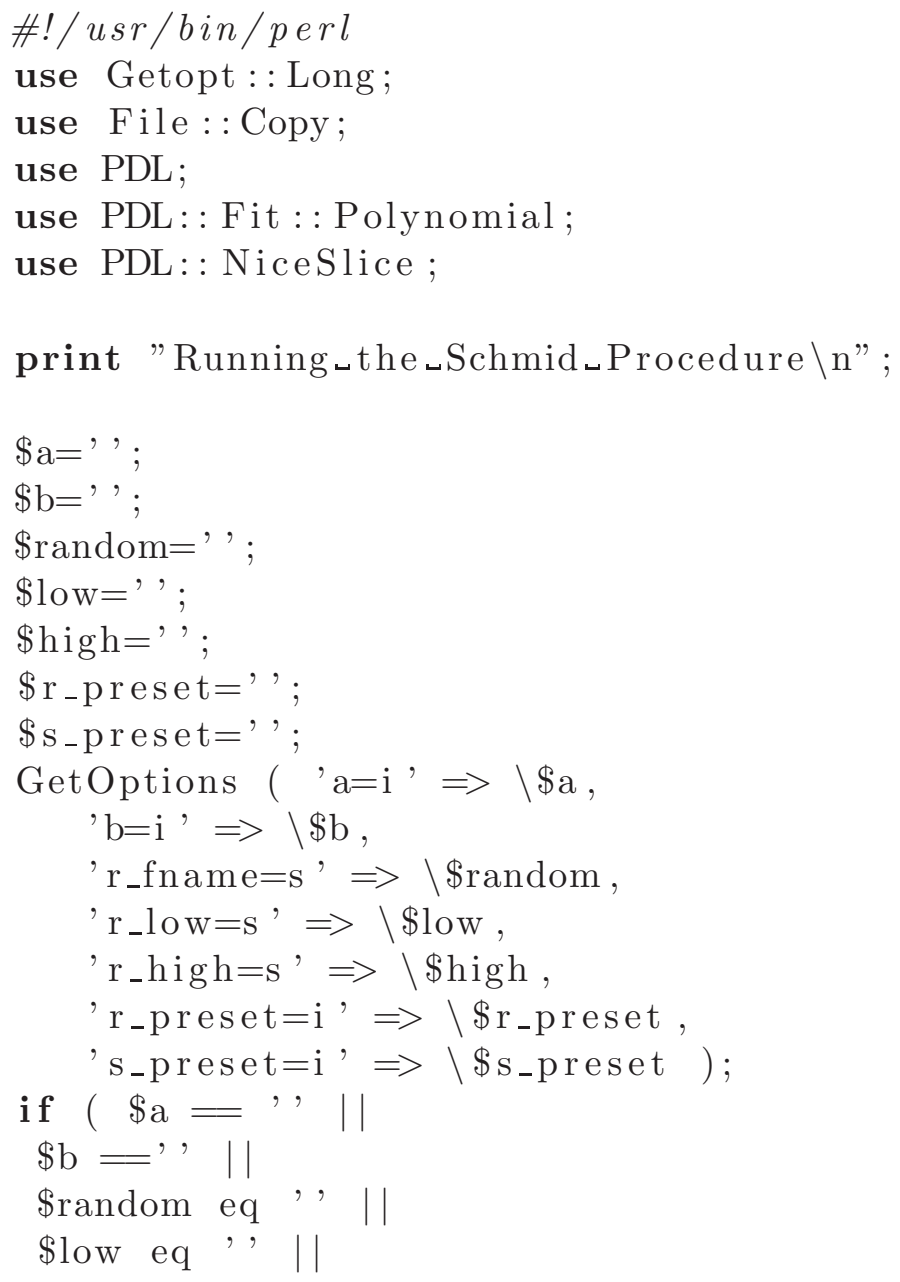


Rev. 1.2

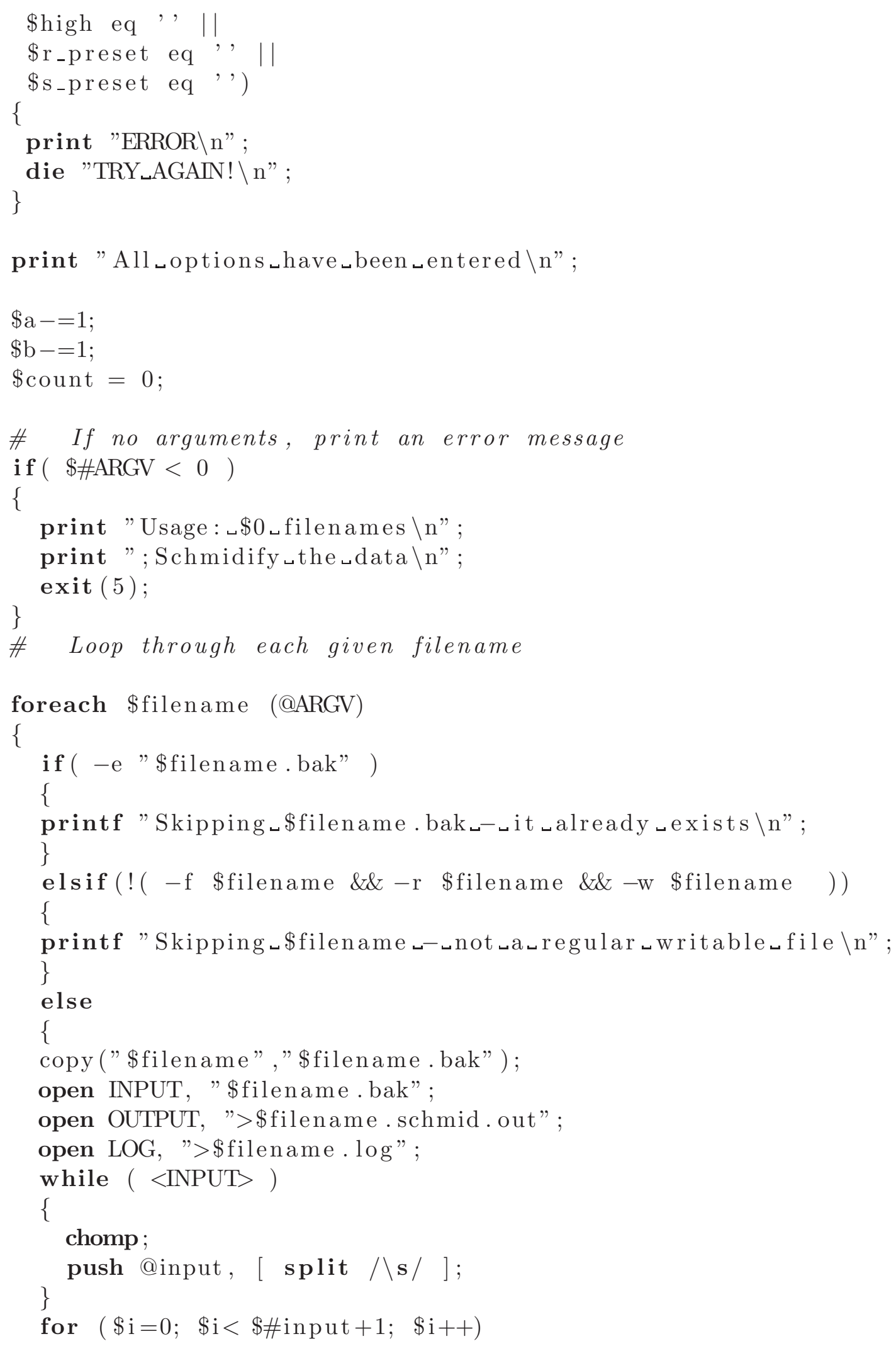


Rev. 1.2

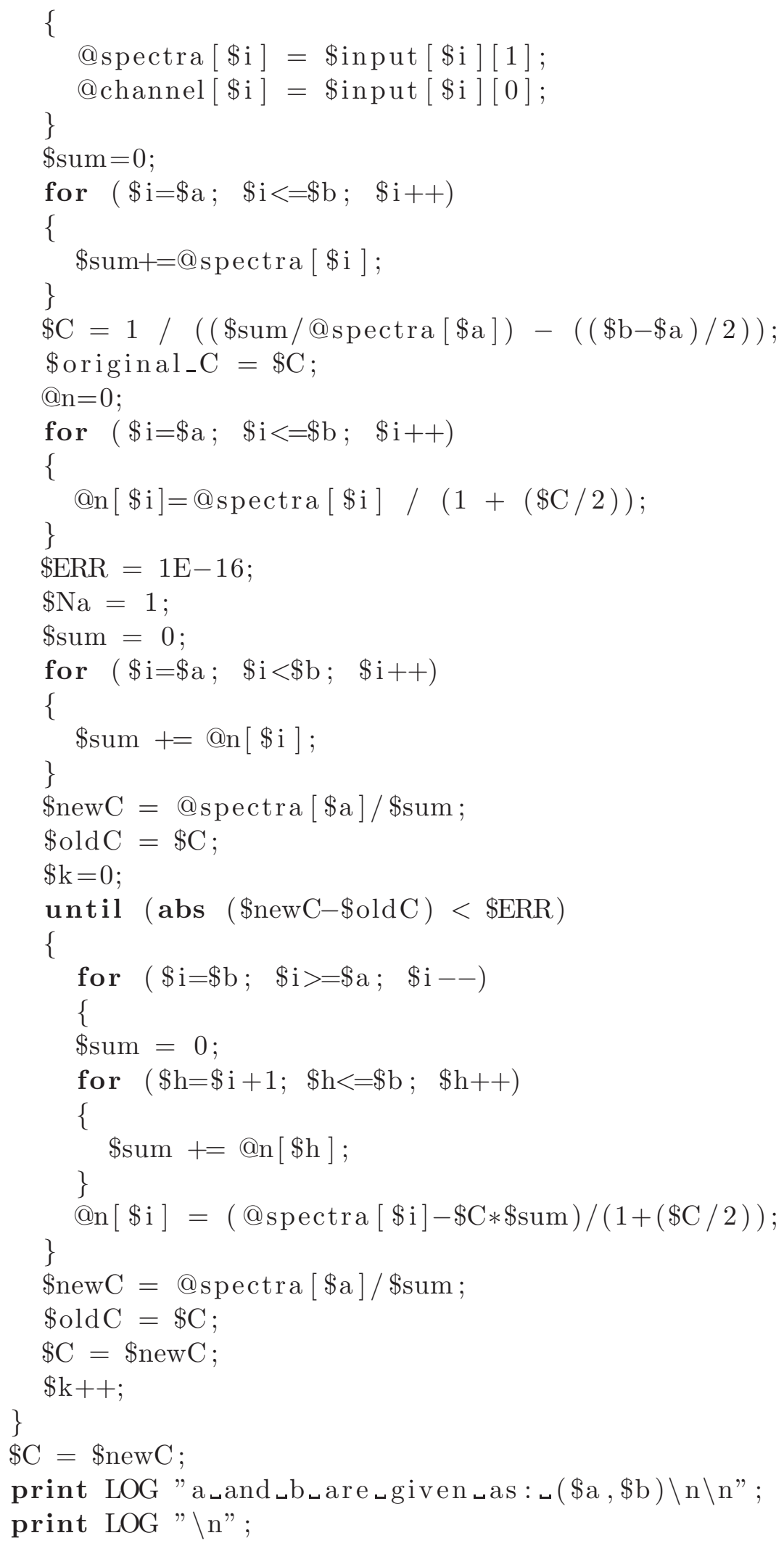




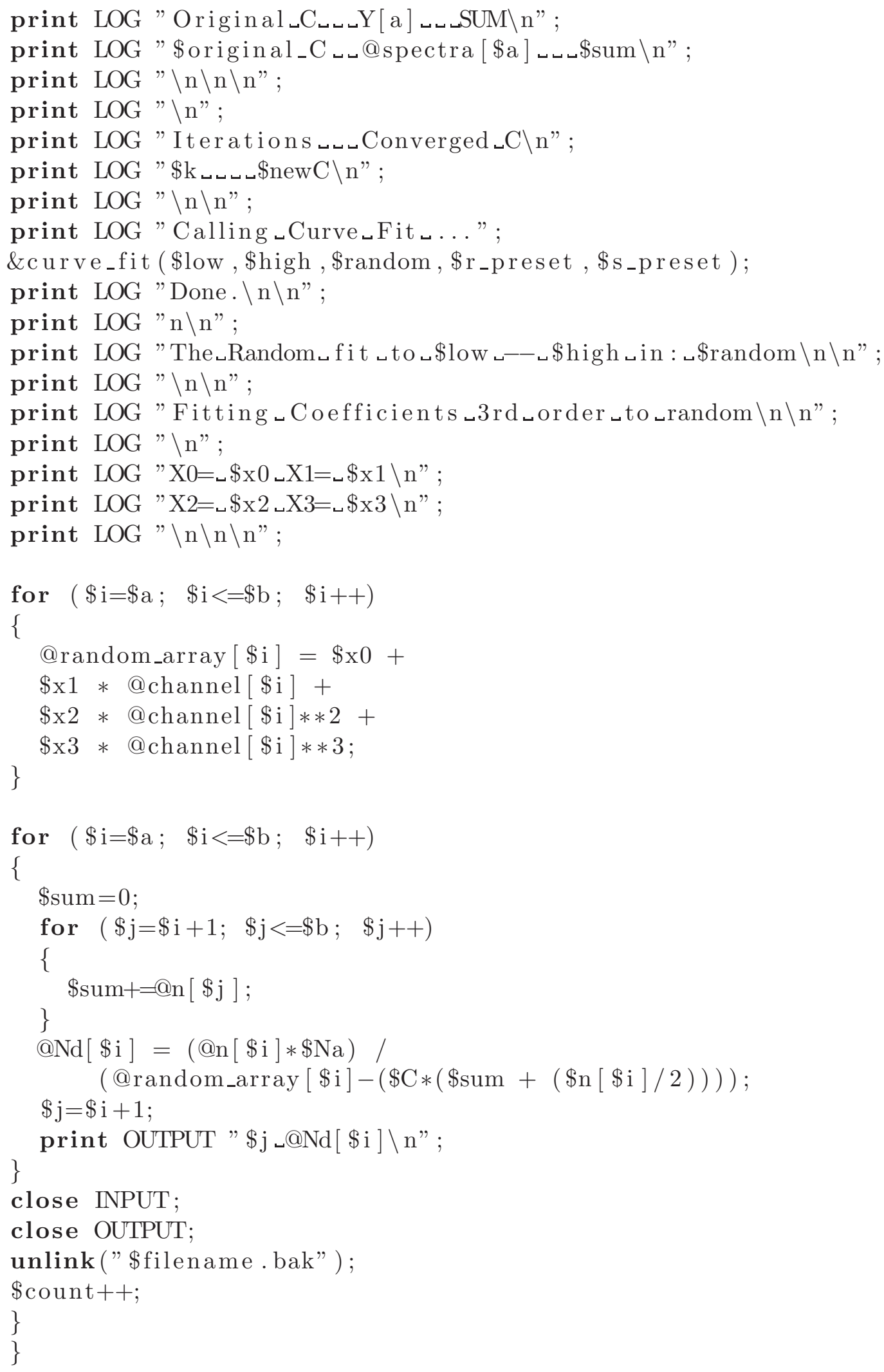


Rev. 1.2

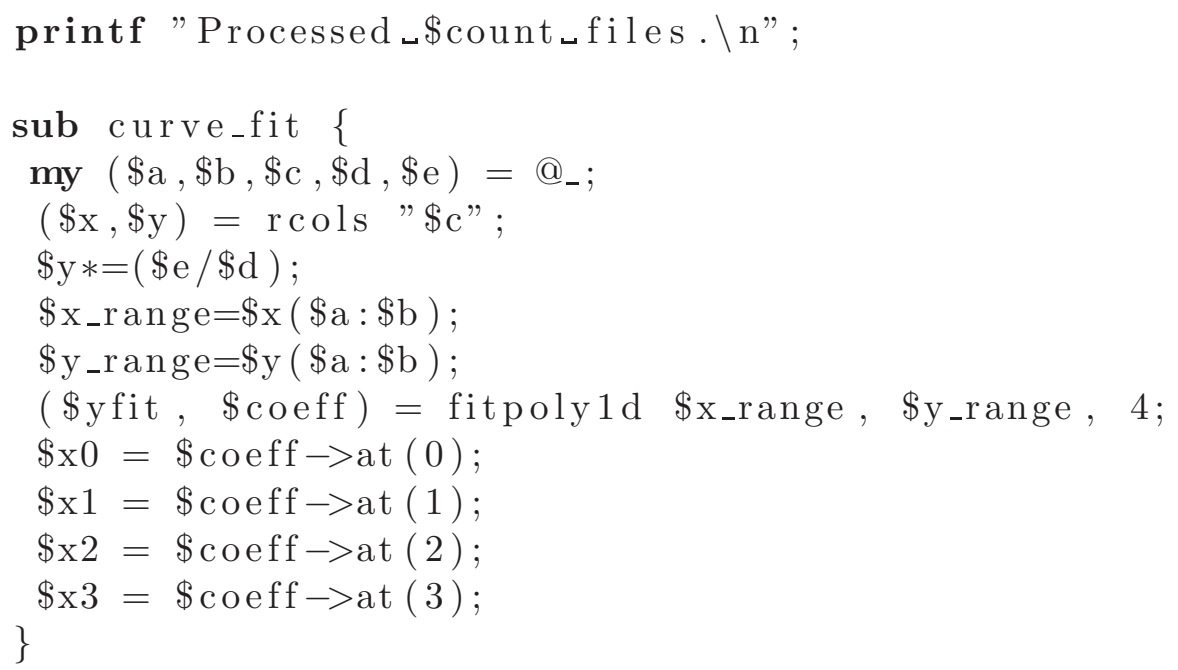


Rev. 1.2

\section{APPENDIX B}

\section{AUSPICES STATEMENT}

This work was performed under the auspices of the U.S. DOE by LLNL under Contract DE-AC52-07NA27344. 